\title{
Spectroscopic study of light Lambda hypernuclei via the $\left(\mathrm{e}, \mathrm{e}^{\prime} \mathrm{K}^{+}\right)$reaction
}

\author{
A Dissertation \\ By \\ YUICHI OKAYASU
}

Submitted to

Department of Physics, Tohoku University

in partial fulfillment of the requirements

for the degree of

DOCTOR OF SCIENCE

September 21, 2008 



\section{Abstract}

Hypernuclear production through the $\left(\mathrm{e}, \mathrm{e}^{\prime} \mathrm{K}^{+}\right)$reaction makes it possible to realize sub-MeV high-resolution spectroscopy of $\Lambda$ hypernuclei thanks to the availability of high quality primary beams. It is in contrast to the $\left(\mathrm{K}^{-}, \pi^{-}\right)$and $\left(\pi^{+}, \mathrm{K}^{+}\right)$reactions which use secondary beams of pions and kaons. Even though hypernuclear cross sections are much smaller than those from meson beams, the (e, $\left.\mathrm{e}^{\prime} \mathrm{K}^{+}\right)$reaction has advantageous features for $\Lambda$ hypernuclear production.

A goal of the present study is to further explore and establish $\left(\mathrm{e}, \mathrm{e}^{\prime} \mathrm{K}^{+}\right)$spectroscopy as a tool to investigate $\Lambda$ hypernuclei, succeeding the pioneering experiment JLab E89-009, which was successfully carried out previously.

A $1.8 \mathrm{GeV}$ electron beam hit targets at the entrance of the splitter magnet in JLab's Hall C. A positive kaon and a scattered electron were deflected by the splitter magnet to opposite directions and guided to the high resolution kaon spectrometer (HKS) and the scattered electron spectrometer (Enge), respectively. The HKS, which has a high momentum resolution $\left(\Delta p / p \sim 2.0 \times 10^{-4}[\mathrm{FWHM}]\right)$ and a large solid angle (16 msr with the splitter), was newly constructed for $\left(\mathrm{e}, \mathrm{e}^{\prime} \mathrm{K}^{+}\right)$hypernuclear spectroscopy. The Enge, which was used in E89-009, was tilted vertically by 8 degrees perpendicular to the splitter dispersion plane in order to suppress Bremsstrahlung and Møller electrons that cause huge backgrounds (tilt method). The present experimental configuration allows us to conduct high luminosity hypernuclear experiments with the $\left(\mathrm{e}, \mathrm{e}^{\prime} \mathrm{K}^{+}\right)$reaction.

Data from three targets, $\mathrm{CH}_{2}\left(465 \mathrm{mg} / \mathrm{cm}^{2}\right),{ }^{7} \mathrm{Li}\left(189 \mathrm{mg} / \mathrm{cm}^{2}\right)$ and ${ }^{12} \mathrm{C}\left(100 \mathrm{mg} / \mathrm{cm}^{2}\right)$, were analyzed in the present study. The elementary processes $\mathrm{p}\left(\mathrm{e}, \mathrm{e}^{\prime} \mathrm{K}^{+}\right) \Lambda / \Sigma^{0}$ were used for calibrating the absolute scale of missing mass. Hypernuclear spectra of ${ }_{\Lambda}^{12} \mathrm{~B}$ and ${ }_{\Lambda}^{7} \mathrm{He}$ were obtained with the best resolution of hypernuclear spectroscopy (490 $\pm 70 \mathrm{keV}, \mathrm{FWHM}$ for ${ }_{\Lambda}^{12} \mathrm{~B}$ ground state doublet).

Prominent peaks corresponding to the states with a $\Lambda$ hyperon in the $s$ and $p$ orbits were observed with improved resolution and statistics from the ones observed in E89-009 experiment. The average binding energy of ${ }_{\Lambda}^{12} \mathrm{~B}$ ground state doublet was determined to be $11.7 \pm 0.1$ (stat.) \pm 0.1 (syst.) $\mathrm{MeV}$, which is consistent with $11.52 \pm 0.35 \mathrm{MeV}$ as measured by the E89-009 experiment. The production count rate of the ${ }_{\Lambda}^{12} \mathrm{~B}$ ground state doublet was $7.0 \times 10^{-4} \mathrm{~Hz}(100$ $\left.\mathrm{mg} / \mathrm{cm}^{2}, I_{e}=20.2 \mu \mathrm{A}\right)$, being much improved from $1.0 \times 10^{-4} \mathrm{~Hz}\left(22 \mathrm{mg} / \mathrm{cm}^{2}, I_{e}=0.66 \mu \mathrm{A}\right)$ in E89-009. The cross section of the ${ }_{\Lambda}^{12} \mathrm{~B}$ ground state doublet, which was integrated over the $\mathrm{K}^{+}$scattering angle $\left(1^{\circ}<\theta_{\mathrm{K}^{+}}<13^{\circ}\right)$, was deduced to be $54 \pm 10$ (stat.) $\pm_{22}^{25}$ (syst.) nb/sr. A theoretical calculation with the DWIA gave a consistent result with the present data.

The ${ }_{\Lambda}^{7}$ He excitation spectrum was observed for the first time with the (e,e' $\mathrm{K}^{+}$) reaction. The binding energy of the ${ }_{\Lambda}^{7} \mathrm{He}$ ground state was found to be $5.1 \pm 0.2$ (stat.) \pm 0.1 (syst.) $\mathrm{MeV}$ and the cross section to be $12 \pm 3$ (stat.) \pm 3 (syst.) $\mathrm{nb} / \mathrm{sr}\left(1^{\circ}<\theta_{\mathrm{K}^{+}}<13^{\circ}\right)$ obtained in the ${ }^{7} \mathrm{Li}\left(\mathrm{e}, \mathrm{e}^{\prime} \mathrm{K}^{+}\right)_{\Lambda}^{7}$ He reaction. A theoretical prediction reproduced the measured cross section of the ${ }_{\Lambda}^{7} \mathrm{He}$ ground state within errors, although the calculated binding energy of the ground state was inconsistent with the experimental data.

The second generation of (e,e' $\left.\mathrm{K}^{+}\right)$hypernuclear spectroscopy experiments proved to be practical and powerful. We have achieved high energy resolution and high yield rate of $\Lambda$ hypernuclear spectroscopy with the $\left(\mathrm{e}, \mathrm{e}^{\prime} \mathrm{K}^{+}\right)$reaction, introducing the HKS spectrometer and the new tilt method. 
A solid ground for future high precision $\Lambda$ hypernuclear spectroscopy with the $\left(\mathrm{e}, \mathrm{e}^{\prime} \mathrm{K}^{+}\right)$reaction has been established. 



\section{Contents}

1 Introduction $\quad 1$

1.1 A $\Lambda$ hyperon, "Probe of the nuclear interior" . . . . . . . . . . . . . 1

1.2 Hyperon-nucleon interaction . . . . . . . . . . . . . . . . . 2

1.3 Historical overview . . . . . . . . . . . . . . . . . . . . . . . . 4

1.4 Hypernuclear production mechanisms . . . . . . . . . . . . . . 7

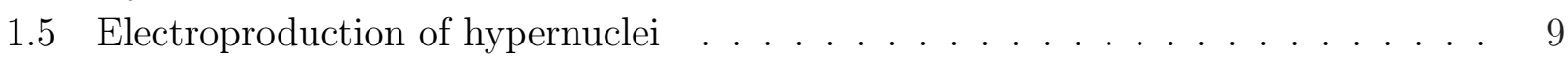

1.5.1 Elementary amplitude and kinematics . . . . . . . . . . . . . 9

1.5.2 Electroproduction of hypernuclei . . . . . . . . . . . . . . 12

1.6 Goal of the present thesis . . . . . . . . . . . . . . . . . . 14

2 Experimental apparatus $\quad 15$

2.1 Overview . . . . . . . . . . . . . . . . . . . . . 15

2.2 Experimental condition . . . . . . . . . . . . . . . . 17

2.2 .1 Kinematics . . . . . . . . . . . . . . . . . 17

2.2 .2 Tilt method principal . . . . . . . . . . . . . . . . . . 21

2.3 The HKS-Enge spectrometer system . . . . . . . . . . . . . . . . . . . . . . . 24

2.3.1 Splitter magnet and target chamber . . . . . . . . . . . . . . . 24

2.3.2 Enge split pole spectrometer (Enge) . . . . . . . . . . . . . . . . 28

2.3.3 High resolution Kaon Spectrometer (HKS) . . . . . . . . . . . . . . 32

2.4 Expected singles rate and energy resolution . . . . . . . . . . . . . . . . . . . . . . . . . . . . . . . . . . .

2.5 Trigger . . . . . . . . . . . . . . . . . . . . . . 39

2.5.1 Grouping trigger . . . . . . . . . . . . . . . . . . 39

2.5.2 Tohoku Universal Logic module (TUL-8040) . . . . . . . . . . . . . 40

2.5.3 Trigger . . . . . . . . . . . . . . . . . . . . . 43

2.6 Data acquisition . . . . . . . . . . . . . . . . . . . . . 44

2.7 Data summary . . . . . . . . . . . . . . . . . . . . . 48

3 Performance of the water Čerenkov counter $\quad 51$

3.1 Overview . . . . . . . . . . . . . . . . . . . . 51

3.2 Design consideration . . . . . . . . . . . . . . . . . . 52

3.2.1 Requirements . . . . . . . . . . . . . . . . . 52

3.2.2 Container design . . . . . . . . . . . . . . . . . . . 54

3.2.3 Reflector and wavelength shifter . . . . . . . . . . . . . . 54

3.3 Performance . . . . . . . . . . . . . . . . . . . . . . . . . . 58

3.3.1 Beam test . . . . . . . . . . . . . . . . . . 58

3.3.2 Performance in the Jlab E01-011 experiment . . . . . . . . . . . . . 60 
4 Data analysis $\quad 65$

4.1 Analysis overview . . . . . . . . . . . . . . . . . . . . . . . 65

4.2 Kaon measurement in $\mathrm{HKS}$. . . . . . . . . . . . . . . . . . . . . . 66

4.2 .1 Particle identification $(\mathrm{PID}) \ldots \ldots \ldots \ldots \ldots$

4.2 .2 HKS tracking . . . . . . . . . . . . . . . . . . . 66

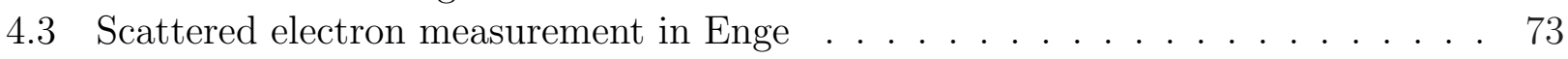

4.4 Two arms coincidence . . . . . . . . . . . . . . . . . . . . 74

4.5 Emission angle and momentum calibration . . . . . . . . . . . . . . . . 77

4.5.1 Analysis overview and flow chart . . . . . . . . . . . . . . 78

4.5.2 Emission angle calibration . . . . . . . . . . . . . . . . . 81

4.5.3 Momentum calibration . . . . . . . . . . . . . . . . . 83

4.6 Systematic errors of mass scale _ . . . . . . . . . . . . . . . . . . . . 84

4.7 Cross section . . . . . . . . . . . . . . . . . . 85

4.7 .1 Acceptance . . . . . . . . . . . . . . . . . . 87

4.7.2 Number of virtual photons . . . . . . . . . . . . . . . . . . . 90

4.7.3 Correction factors . . . . . . . . . . . . . . . . . . . . . . . . . . . . . . . . .

4.7.4 Systematic errors . . . . . . . . . . . . . . . . . . . . . 104

4.7 .5 Energy resolution . . . . . . . . . . . . . . . . 105

5 Experimental results and discussion $\quad 107$

5.1 Experimental results . . . . . . . . . . . . . . . . . . 107

5.1 .1 Hypernuclear mass spectra . . . . . . . . . . . . . . . . 107

5.1.2 Effectiveness of the present experimental configuration . . . . . . . . . 111

5.2 Discussion . . . . . . . . . . . . . . . . . . . . . 111

5.2.1 Spectroscopy of ${ }_{\Lambda}^{12} \mathrm{~B}$ hypernuclei . . . . . . . . . . . . . 112

5.2 .2 Spectroscopy of ${ }_{\Lambda}^{7} \mathrm{He}$ hypernuclei . . . . . . . . . . . . . . . 118

$\begin{array}{llr}6 & \text { Conclusion } & 129\end{array}$

$\begin{array}{ll}\text { A Mass references used in spectra calculation } & 133\end{array}$

B S-factors for proton pick-up reactions 135

C Additional information of the water Čerenkov counter 137

C.1 Aging test for number of photoelectron . . . . . . . . . . . . . 137

D Systematic errors of the ${ }_{\Lambda}^{12}$ B cross section $\quad 139$ 


\section{List of Figures}

1.1 The octet of light $J^{\pi}=1 / 2^{+}$baryons . . . . . . . . . . . . . . . . 1

$1.2 A$ dependence of the binding energies of $\Lambda$ single particle states . . . . . . . . 3

1.3 The first observation of a $\Lambda$ hypernucleus in a photographic emulsion . . . . . 5

1.4 The $\Lambda$ hypernuclear chart . . . . . . . . . . . . . . . 7

1.5 Hypernuclear production mechanism . . . . . . . . . . . . . 8

1.6 Hyperon recoil momenta as a function of projectile momentum . . . . . . . . . 8

1.7 Kinematics of the electroproduction. . . . . . . . . . . . . . . . . 10

2.1 Schematic of CEBAF and experimental halls at JLab . . . . . . . . . . . . 16

2.2 Hall C beamline . . . . . . . . . . . . . . . . . . . . . . 16

2.3 Schematic drawing of the $\left(\mathrm{e}, \mathrm{e}^{\prime} \mathrm{K}^{+}\right)$reaction $\ldots \ldots \ldots \ldots \ldots$

2.4 Total cross section of the $\mathrm{p}\left(\gamma, \mathrm{K}^{+}\right) \Lambda$ reaction . . . . . . . . . . . . . . . 18

2.5 The photoproduction cross section of ${ }_{\Lambda}^{12} \mathrm{~B}$ ground state with a DWIA calculation 18

2.6 Angular distribution of the virtual photon flux . . . . . . . . . . . . . . . 19

2.7 Momentum acceptance of the kaon and scattered electron spectrometers . . . . . 20

2.8 Schematic comparison of the splitter and Enge configuration in E89-009 and

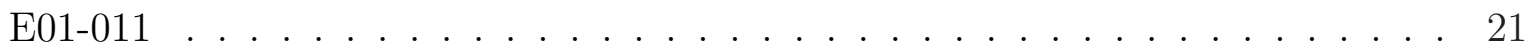

$2.9{ }_{\Lambda}^{12} \mathrm{~B}$ spectrum measured in the JLab E89-009 experiment . . . . . . . . . . . . 21

2.10 Enge tilt angle dependence of the expected rate . . . . . . . . . . . . . . 22

2.11 Tilt angle effects on the electron singles rates . . . . . . . . . . . . . . . . . 23

2.12 JLab E01-011 experimental setup . . . . . . . . . . . . . . . . . . . . . . . . . . . . . . . . . . . . .

2.13 Schematic design of the splitter magnet . . . . . . . . . . . . . . . . . . 25

2.14 Schematic and field map comparison of the splitter magnet . . . . . . . . . 26

2.15 Schematic drawing of the Enge . . . . . . . . . . . . . . . . . 28

2.16 Enge BI excitation curve (measured) . . . . . . . . . . . . . . . . . . . . . . . . . . . 39

2.17 Enge BI excitation curve (calculated) . . . . . . . . . . . . . . . . . 30

2.18 A multiple scattering angle dependence on $\Delta p$ of the Enge $\ldots \ldots \ldots 31$

2.19 Mechanical drawing of the Enge Drift Chamber . . . . . . . . . . . . . . . . . 32

2.20 Schematic of Enge hodoscope . . . . . . . . . . . . . . . . . . . . . . . . . . . . . . . . . . . .

2.21 Layer configuration of the HKS drift chamber . . . . . . . . . . . . . . . . . . . . . . . . . . . 34

2.22 The geometry of the HKS drift chamber . . . . . . . . . . . . . . . . . . . . . 35

2.23 HKS hodoscope HTF1X . . . . . . . . . . . . . . . . . . . 36

2.24 HKS hodoscope HTF1Y . . . . . . . . . . . . . . . . . . . . . . . . . . . . . . . . . . . . . . . . . .

2.25 HKS aerogel Čerenkov counter . . . . . . . . . . . . . . . . . . . 37

2.26 Schematics of a grouping trigger for HKS detector package . . . . . . . . . . . 40

2.27 Grouping definition for HTOF1X and 2X . . . . . . . . . . . . . . . 41

2.28 Front and top view of the TUL-8040 . . . . . . . . . . . . . . . . . 42

2.29 The HKS trigger logic . . . . . . . . . . . . . . . . . . . . . . . . . . . . . . . . . . . . . . 43

2.30 The Trigger Supervisor $(\mathrm{TS})$ logic . . . . . . . . . . . . . . . . . 44 
2.31 A flowchart of CODA . . . . . . . . . . . . . . . . . . 45

3.1 Top view of the E01-011 experimental setup . . . . . . . . . . . . . . . 52

3.2 Particle momentum dependence of the number of photons . . . . . . . . . 53

3.3 Schematic drawing of the water Čerenkov counter . . . . . . . . . . . . . . . 54

3.4 Schematic view of the spectrophotometer . . . . . . . . . . . . . . 55

3.5 Absolute reflectance of the barium sulfate . . . . . . . . . . . . . . 56

3.6 Relative diffusion reflectance for various materials . . . . . . . . . . . . . . 57

3.7 Density dependence of the amino G-salt $\mathrm{pH} \ldots \ldots \ldots$. . . . . . . . . 58

3.8 Schematic view of the KEK-PS T1 beam line . . . . . . . . . . . . . . . . . . 59

3.9 Schematic top view of the counter test performed at KEK . . . . . . . . . . . 59

3.10 Time of flight distribution with particle momentum $1.2 \mathrm{GeV} / c \ldots$. . . . . . 60

3.11 Number of photoelectron comparison with pure water and chemical water . . . . 61

3.12 Amino G-salt density dependence on measured ratio of photoelectrons . . . . . . 61

3.13 An experimental term dependence of the number of photoelectrons . . . . . . 62

3.14 WČ normalized number of photoelectrons . . . . . . . . . . . . . . . . 63

3.15 WČ cut efficiency . . . . . . . . . . . . . . . . . . . 63

4.1 E01-011 analysis flow chart . . . . . . . . . . . . . . . 65

4.2 A typical distribution of residual and tracking $\chi^{2}$ per d.o.f of the HDC . . . . 67

4.3 A typical particle velocity $\beta$ resolution $\ldots \ldots \ldots$. . . . . . . . . . . 68

4.4 Particle identification with TOF measurement . . . . . . . . . . . . . . 69

4.5 A scatter plot for proton rejection . . . . . . . . . . . . . . . . 70

4.6 Precise kaon identification with the target time spectrum . . . . . . . . . 71

4.7 The target time cuts effect for kaon selection with $\beta_{\mathrm{TOF}}-\beta_{\mathrm{K}^{+}}$spectrum $\ldots . .72$

4.8 Scatter plot for pion and positron rejection . . . . . . . . . . . . . . 72

$4.9 \beta_{\mathrm{TOF}}-\beta_{\mathrm{K}^{+}}$spectra with/without Čerenkov cut . . . . . . . . . . . . 73

4.10 Horizontal coordinates correrations of Enge focal plane and the Enge hodoscopes 74

4.11 Coincidence time spectra . . . . . . . . . . . . . . . . . 75

4.12 Principle of the mixed event analysis . . . . . . . . . . . . . . 76

4.13 Effectiveness of the mixed event analysis . . . . . . . . . . . . . . 77

4.14 Momenta and emission angles definition for mass spectrum calculation . . . . 78

4.15 A flow chart for mass reconstruction . . . . . . . . . . . . . . . . . . 79

4.16 Enge and HKS collimator and sieve slit . . . . . . . . . . . . . . . . . 80

4.17 The flow chart for emission angle calibration procedure . . . . . . . . . . 81

4.18 The reconstructed Enge and HKS S.S. image . . . . . . . . . . . . . . . . . 82

4.19 Comparison of the Enge and HKS emission angle with/without S.S. calibration . 83

4.20 The flow chart for the momentum calibration procedure . . . . . . . . . . . . 84

4.21 Missing mass spectra $\left(M_{X}-M_{\Lambda}\right)$ of $\mathrm{p}\left(\mathrm{e}, \mathrm{e}^{\prime} \mathrm{K}^{+}\right) \Lambda / \Sigma^{0}$ for $\mathrm{CH}_{2}$ runs. . . . . . . . 86

4.22 Reconstructed HKS and Enge emission angles . . . . . . . . . . . . . . . 87

4.23 Calculated Enge and HKS momentum correlation . . . . . . . . . . . . . 88

4.24 Reconstructed Enge and HKS momentum correlations for $\mathrm{CH}_{2}$ and ${ }^{12} \mathrm{C}$ target data . . . . . . . . . . . . . . . . . . . . 89

4.25 Momentum dependence of the Enge/HKS solid angle . . . . . . . . . . . . . . 90

4.26 The integrated virtual photons . . . . . . . . . . . . . . . . . . 91

$4.27 \beta_{\mathrm{TOF}}$ spectrum without any offline Čerenkov cut. . . . . . . . . . . . . . . . . 92

4.28 HKS / Enge tracking procedure. . . . . . . . . . . . . . . . . . . . 94

4.29 Run dependence of the HKS / Enge tracking efficiency. . . . . . . . . . . . . . . . 96

4.30 Correlation of kaon survival ratio and AČ threshold . . . . . . . . . . . . . . 97 
4.31 Correlation of kaon survival ratio and WČ threshold . . . . . . . . . . . . . 98

$4.32 \beta$ cut efficiency . . . . . . . . . . . . . . . . . . . . . . . . 99

4.33 The momentum dependence of kaon absorption . . . . . . . . . . . . . . . 102

4.34 Momentum dependence of kaon decay factor . . . . . . . . . . . . . . . . . . . . . . . . . . . . . . . . . . . . . . . .

4.35 Computer live time factor . . . . . . . . . . . . . . . . 104

5.1 Hypernuclear mass spectra for ${ }_{\Lambda}^{12}$ B . . . . . . . . . . . . . . . . . . 109

5.2 Hypernuclear mass spectra for ${ }_{\Lambda}^{7} \mathrm{He} \ldots \ldots \ldots \ldots$

5.3 Comparison between E89-009 and E01-011 ${ }_{\Lambda}^{12} \mathrm{~B}$ spectra . . . . . . . . . . . 112

5.4 A comparison of the hypernuclear mass spectra for $A=12 \ldots \ldots$. . . . . . 114

5.5 A calculated hypernuclear mass spectra of ${ }_{\Lambda}^{12} \mathrm{~B} \ldots \ldots \ldots$

$5.6 \Lambda \mathrm{N}-\Sigma \mathrm{N}$ coupling in three-body interaction on ${ }_{\Lambda}^{4} \mathrm{He} \ldots \ldots . \ldots . . \ldots 118$

5.7 Binding energies comparison for ${ }_{\Lambda}^{4} \mathrm{H}$ and ${ }_{\Lambda}^{4} \mathrm{He} \ldots \ldots$. . . . . . . . . . . . . 119

5.8 Calculated energies of the low-lying states of $A=7 ; T=1$ isospin-triplet $\Lambda$ hypernuclei and measured binding energies of ${ }_{\Lambda}^{7} \mathrm{Be}$ and ${ }_{\Lambda}^{7} \mathrm{Li}$. . . . . . . . . . . 120

5.9 Hypernuclear mass spectra comparison with $\left(\mathrm{e}, \mathrm{e}^{\prime} \mathrm{K}^{+}\right)$and $\left(\pi^{+}, \mathrm{K}^{+}\right)$reaction for

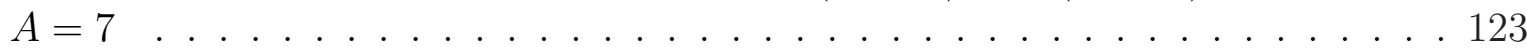

5.10 Theoretical calculations of the ${ }_{\Lambda}^{7} \mathrm{He}$ cross sections . . . . . . . . . . . 125

5.11 Models of the $A=7$ triplets with the four-body cluster calculation . . . . . . 126

5.12 Theoretical and experimental energy scheme comparison for the ${ }_{\Lambda}^{7} \mathrm{He}$. . . . . . 127

5.13 Comparison between theoretical and experimental energy scheme for the $A=7$ isospin-triplet $\Lambda$ hypernuclei . . . . . . . . . . . . . . . . . 128

B.1 Relative spectroscopic factors for proton pick-up reactions on ${ }^{7} \mathrm{Li}$. . . . . . . . 135

B.2 Relative spectroscopic factors for proton pick-up reactions on ${ }^{12} \mathrm{C} \ldots . . . .136$

C.1 Schematic of cosmic ray bench test for chemical stability test . . . . . . . . 137

C.2 Life time of the number of photoelectrons for the Amino G-salt water Cerenkov counter . . . . . . . . . . . . . . . . . . 138

D.1 The systematic errors estimation of the cross section for the ${ }_{\Lambda}^{12} \mathrm{~B}$ ground state doublet . . . . . . . . . . . . . . . . . . . 140 



\section{List of Tables}

2.1 E01-011 experimental condition and spectrometer specification . . . . . . . . 25

2.2 Splitter magnet parameters . . . . . . . . . . . . . . . . . . . 27

2.3 The list of targets used in E01-011. . . . . . . . . . . . . . . . . 27

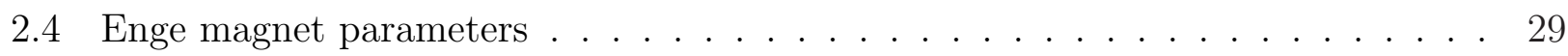

2.5 HDC parameters . . . . . . . . . . . . . . . . . . . . 30

2.6 HKS magnet parameters . . . . . . . . . . . . . . . . . . . . . . . 33

2.7 HDC parameters . . . . . . . . . . . . . . . . . . . . . . . . 35

2.8 HTOF parameters . . . . . . . . . . . . . . . . . . . 37

2.9 Estimated singles rates . . . . . . . . . . . . . . . . . . 38

2.10 Expected energy resolution for bound states in ${ }_{\Lambda}^{7} \mathrm{He}$ and ${ }_{\Lambda}^{12} \mathrm{~B}$ mass spectra . . . . 39

2.11 Energy loss summary for various targets . . . . . . . . . . . . . . . . . . . . . . . . . . . . .

2.12 Typical ENGE, HKS and Coincidence trigger rate. . . . . . . . . . . . . . . . . . . . . . . . . . . 46

2.13 Data acquisition settings . . . . . . . . . . . . . . . . 48

2.14 Run summary . . . . . . . . . . . . . . . . . . . . . 50

3.1 The water Čerenkov counter parameters . . . . . . . . . . . . . 55

3.2 Specification of the spectrophotometer . . . . . . . . . . . . 56

3.3 The measured and expected HKS singles rates . . . . . . . . . . . . . . . . 62

4.1 Fitting results of $\Lambda$ and $\Sigma^{0}$ in the missing mass spectra. . . . . . . . . . . . 85

4.2 List of the experimental efficiency factors and typical values. . . . . . . . . . 87

4.3 A summary of the number of kaons for each data set . . . . . . . . . . . . . 93

4.4 A summary of the AČ cut efficiencies . . . . . . . . . . . . . . . . . . 97

4.5 A summary of the WČ cut efficiencies . . . . . . . . . . . . . . . . . . 98

4.6 A summary of the $\beta_{\mathrm{TOF}}-\beta_{\mathrm{K}^{+}}$cut efficiencies for each data set . . . . . . . . . 100

4.7 Properties of HKS materials for kaon absorption factor estimation . . . . . . . . 101

4.8 Absorption factors depending on target . . . . . . . . . . . . . . 102

$4.9 \mathrm{~K}^{+}$decay channels . . . . . . . . . . . . . . . . . . . . . . . . . . . . . . . . . . . . . . . . .

4.10 A summary of systematic errors for the cross sections . . . . . . . . . . . . 104

5.1 Data sets used for the analysis of the ${ }_{\Lambda}^{7} \mathrm{He}$ and ${ }_{\Lambda}^{12} \mathrm{~B} \ldots \ldots . \ldots . \ldots . \ldots 108$

5.2 Comparison of the measured performance between E01-011 and E89-009 . . . 111

5.3 Results of fitting the ${ }_{\Lambda}^{12} \mathrm{~B}$ spectrum . . . . . . . . . . . . . . . 113

5.4 Measured excitation, binding energies and cross sections of ${ }_{\Lambda}^{12} \mathrm{C}$ spectrum (KEK

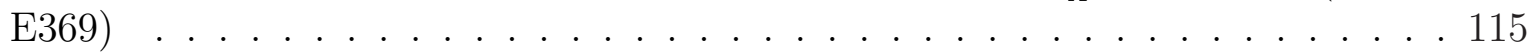

$5.5 s-p$ states energy spacing obtained from the ${ }_{\Lambda}^{12} \mathrm{C}$ and ${ }_{\Lambda}^{12} \mathrm{~B}$ excitation spectra . . 115

5.6 Comparison of obtained experimental results with theoretical calculations for ${ }_{\Lambda}^{12} \mathrm{~B} 116$

5.7 Comparison of obtained experimental results with theoretical calculations for ${ }_{\Lambda}^{12} \mathrm{~B} 116$

5.8 Results of fitting the ${ }_{\Lambda}^{7} \mathrm{He}$ spectrum . . . . . . . . . . . . . . . . . 121 
5.9 Measured excitation, binding energies and cross sections of ${ }_{\Lambda}^{7} \mathrm{Li}$ spectrum (KEK E336) . . . . . . . . . . . . . . . . . . . . 121

5.10 Comparison of experimental result of ${ }^{7} \mathrm{Li}\left(\mathrm{e}, \mathrm{e}^{\prime} \mathrm{K}^{+}\right){ }_{\Lambda}^{7} \mathrm{He}$ cross section for the ground state with theoretical predictions . . . . . . . . . . . . . . . 124

5.11 Comparison of experimental result of the ${ }_{\Lambda}^{7} \mathrm{He}$ ground state binding energy with theoretical prediction . . . . . . . . . . . . . . 126

A.1 Values of particle and target nuclear masses. . . . . . . . . . . . . 133

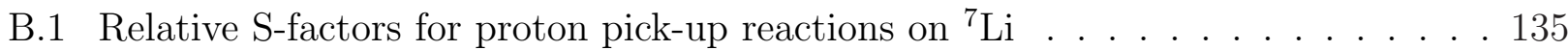

B.2 Relative S-factors for proton pick-up reactions on ${ }^{12} \mathrm{C} \ldots \ldots . . \ldots 136$ 


\section{Chapter 1}

\section{Introduction}

\subsection{A $\Lambda$ hyperon, "Probe of the nuclear interior"}

A hyperon, such as $\Lambda, \Sigma$ and $\Xi$, is a baryon, like the neutron and proton, with spin $J=1 / 2$. However it consists of not only up and down quarks but also strange quarks. Fig. 1.1 shows a baryon octet with the strangeness degree of freedom.

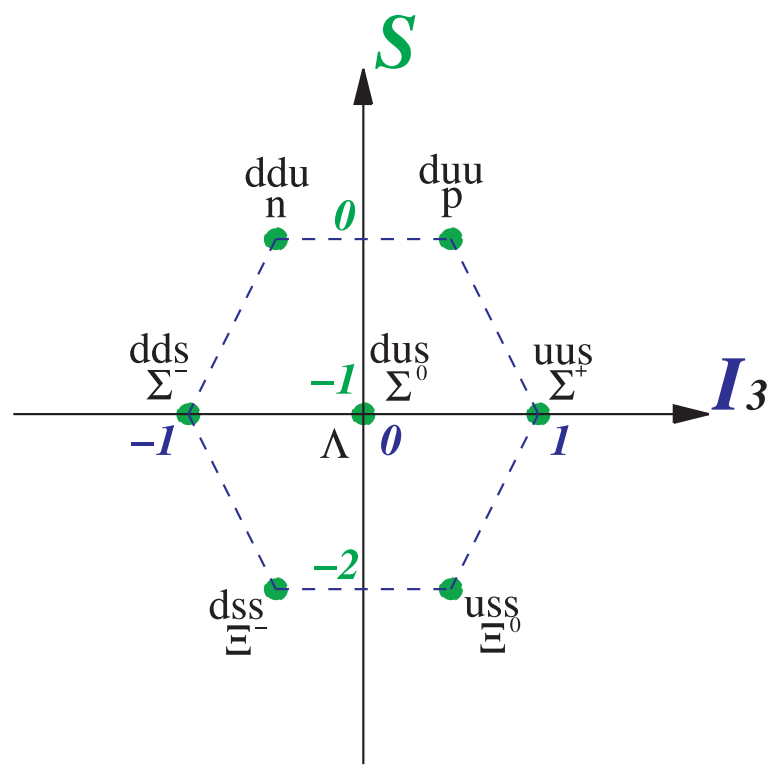

Figure 1.1: The octet of light $J^{\pi}=1 / 2^{+}$baryons on the third component of isospin $I_{3}$ and strangeness $S$ plane.

A hypernucleus is formed by replacing one or more of the nucleons in a normal nucleus by hyperons. Since a hyperon contains at least one strange quark, it can be distinguished from ordinary nucleons, and is not affected by the Pauli principle from other nucleons in the nucleus. Thus a hyperon provides us with a way to investigate the interior structure of a nucleus which cannot be accessed by excitation of a normal nucleus.

Generally, $\Lambda$ hypernuclear states, which are usually generated by introducing at least one $\Lambda$ particle into a nucleus, have narrow widths compared to those of the ordinary nuclei.

For example, Bandō et al. [1] discussed that $\Lambda$ bound states have narrow spreading widths of less than a few $100 \mathrm{keV}$ while nucleon deep hole states have a few $10 \mathrm{MeV}$. This is because 
(1) the $\Lambda \mathrm{N}$ interaction is weaker than the $\mathrm{NN}$ interaction,

(2) the $\Lambda \mathrm{N}$ spin-spin interaction is weak and therefore spin vector $(J=1) \mathrm{p}_{\mathrm{N}}-h_{\mathrm{N}}$ excitations are suppressed,

(3) a $\Lambda$ with zero isospin can excite only isoscaler $\mathrm{p}_{\mathrm{N}^{-}} h_{\mathrm{N}}$ modes of the core nucleus, and

(4) no exchange term with nucleons is required.

These characteristics make the width of $\Lambda$ hypernuclear states considerably narrower than the ordinary nucleus for the states at the same excitation energy. In the case of ${ }^{40} \mathrm{Ca}$, for example, H. Bandō et al. predicted that $\Gamma_{\Lambda}(1 s, 0 d) / \Gamma_{\mathrm{N}}(1 s)=0.03-0.07$.

The widths are expected to be much smaller than the energy spacings between the $\Lambda$ major shells and consequently, $\Lambda$ hypernuclear states should be observed with reasonably narrow peaks. This makes a basis for reaction spectroscopy of $\Lambda$ hypernuclei with resolution as good as a few $100 \mathrm{keV}$ for a wide range of excitation.

So far, the energy levels of $\Lambda$ hypernuclear states have been measured through the $\left(\mathrm{K}^{-}, \pi^{-}\right)$ strangeness-exchange reaction and the $\left(\pi^{+}, \mathrm{K}^{+}\right)$associated production reaction with nuclear targets ranging from ${ }^{4} \mathrm{He}$ to ${ }^{209} \mathrm{Bi}$. These experiments were mostly carried out at the proton synchrotron of the European Organization for Nuclear Research (CERN-PS), the alternating gradient synchrotron of the Brookhaven National Laboratory (BNL-AGS) and the $12 \mathrm{GeV}$ proton synchrotron of the High Energy Accelerator Organization (KEK-PS). In particular, the $\left(\pi^{+}, \mathrm{K}^{+}\right)$reaction, which transfers large momentum to recoil hypernuclei, populates a well defined series of $\Lambda$ single-particle hypernuclear states with a $\Lambda$ hyperon in various orbital angular momentum as $s, p, d$, etc. of the $\Lambda$ hyperon.

The binding energies $B_{\Lambda}$ up to the $g$-shell have been extracted from a part of these above data as shown in Fig. 1.2 [2]. By these data, the depth of a local Woods-Saxon ( $\Lambda$-nucleus) potential well was determined to be $\sim 30 \mathrm{MeV}$. Millner et al. [2] provided a description of the binding energy data with a phenomenological $\Lambda$-nucleus potential based on a spherical Skyrme Hartree-Fock approach as shown in solid curves in Fig. 1.2.

However, it is almost impossible to investigate such deeply bound energy states of ordinary nuclei because the spreading width of these states are quite large.

\subsection{Hyperon-nucleon interaction}

Hyperons and nucleons are baryons. The properties of baryon-baryon interactions are still not understood well. Study of the hyperon-nucleon (YN) interaction will provide us with information on the relation of $\mathrm{YN}$ and $\mathrm{NN}$ forces and lead us to a unified understanding of baryon-baryon interactions.

Although scattering experiments should be useful for the investigation, hyperon beams are not readily available since hyperon lifetimes are quite short $\left(\sim 10^{-10} \mathrm{~s}\right)$. Therefore, the YN scattering data are very limited in scope and accuracy.

Investigating the bound hyperon state, i.e. the hypernucleus, and comparing experimental information with theoretical models are ways to extract information about the YN interaction. Hence hypernuclear structure information observed through hypernuclear spectroscopy plays a key role in the investigation of the $\mathrm{YN}$ interaction.

Through $\Lambda$ hypernuclear production experiments over the last decades, it has been found that the weak coupling model which assumes that the $\Lambda$ couples weakly to the ground and excited 


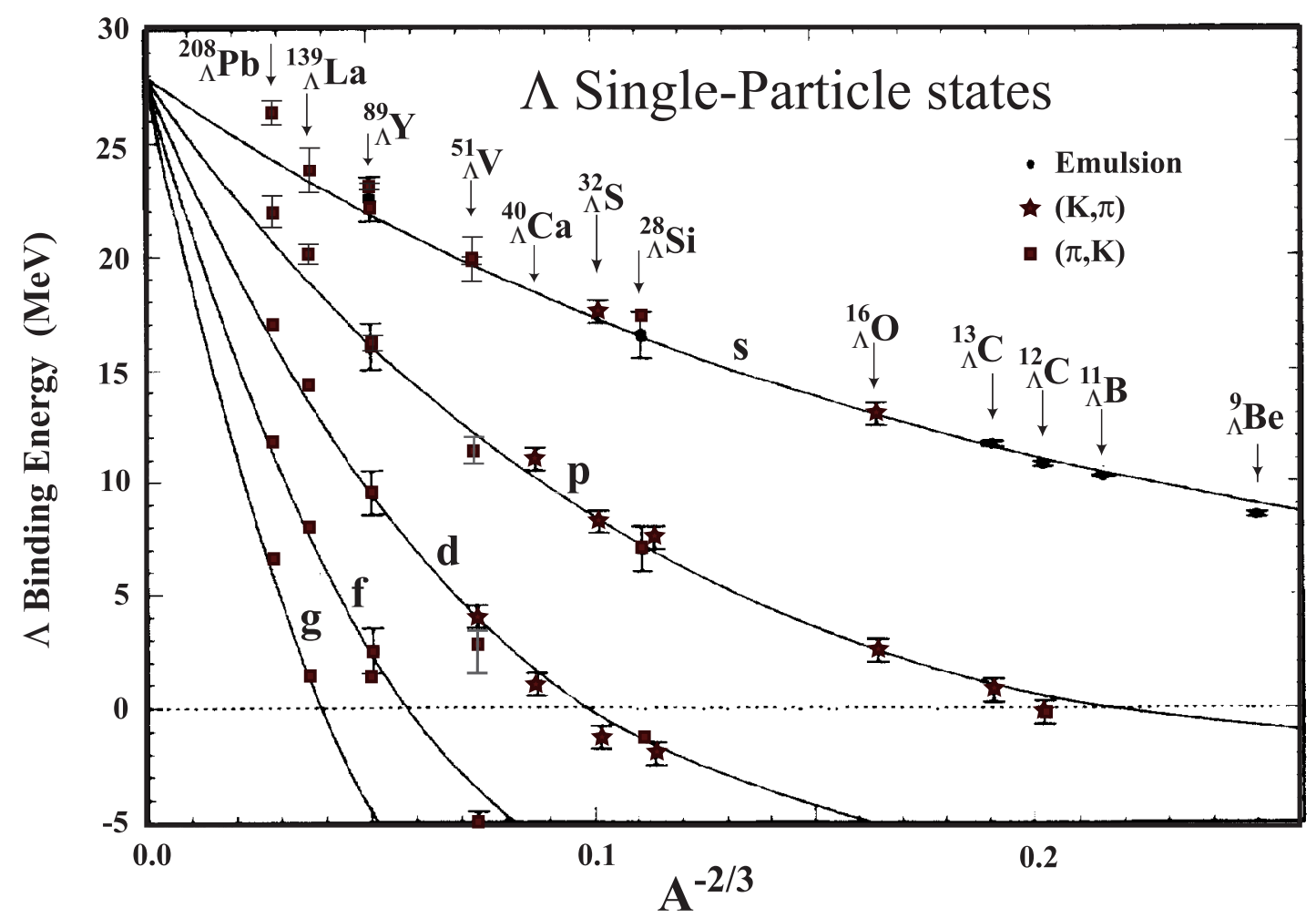

Figure 1.2: The binding energies of $\Lambda$ single particle states as a function of $A^{-2 / 3}$, where $A$ is the mass number of the core nucleus [2]. The data were obtained from in-flight $\left(\pi^{+}, \mathrm{K}^{+}\right)$and $\left(\mathrm{K}^{-}, \pi^{-}\right)$ reactions and emulsion data. The solid curves were provided by the spherical Skyrme Hartree-Fock approach [3]. 
states of the core nucleus reproduces observed hypernuclear level structures [4]. The hypernuclear Hamiltonian [5]:

$$
H=H_{\mathrm{N}}+H_{\Lambda}+V_{\Lambda \mathrm{N}}
$$

is diagonalized in the weak-coupling basis. $H_{\mathrm{N}}$ is the Hamiltonian for the nucleus core due to Cohen and Kurath [6]. $H_{\Lambda}$ is the $\Lambda$ single-particle Hamiltonian, defined by a set of singleparticle energies. The remaining term $V_{\Lambda \mathrm{N}}$ represents the $\Lambda \mathrm{N}$ interaction. $V_{\Lambda \mathrm{N}}$ is often expressed in phenomenological effective YN interactions by the G-matrix method starting from the twobody interaction in free space. Many models of the effective YN interaction generated by Rijken and the Nijmegen group [7] originated with One-Boson-Exchange (OBE) models.

For the case of $p$-shell hypernuclei with a $\Lambda$ in an $s$ orbit, the $p_{\mathrm{N}} s_{\Lambda}$ interaction can be described in terms of five radial integrals $\bar{V}, \Delta, S_{\Lambda}, S_{\mathrm{N}}$ and $T$, assumed to be constant across the $p$-shell and associated with the average central, spin-spin, $\Lambda$ spin-orbit, induced nucleon spin-orbit and tensor terms in the potential [8-10] as:

$$
V_{\Lambda \mathrm{N}}(r)=V_{0}(r)+V_{\sigma}(r) \boldsymbol{s}_{\Lambda} \cdot \boldsymbol{s}_{\mathrm{N}}+V_{\Lambda}(r) \boldsymbol{l}_{\Lambda \mathrm{N}} \cdot \boldsymbol{s}_{\Lambda}+V_{\mathrm{N}}(r) \boldsymbol{l}_{\Lambda \mathrm{N}} \cdot \boldsymbol{s}_{\mathrm{N}}+V_{T}(r) S_{12},
$$

where $\boldsymbol{l}_{\Lambda \mathrm{N}}$ is the relative orbital angular momentum and

$$
S_{12}=3\left(\boldsymbol{\sigma}_{\mathrm{N}} \cdot \hat{\boldsymbol{r}}\right)\left(\boldsymbol{\sigma}_{\Lambda} \cdot \hat{\boldsymbol{r}}\right)-\boldsymbol{\sigma}_{\mathrm{N}} \cdot \boldsymbol{\sigma}_{\Lambda}
$$

with $\hat{\boldsymbol{r}}=\left(\boldsymbol{r}_{\Lambda} \times \boldsymbol{r}_{\mathrm{N}}\right) /\left|\boldsymbol{r}_{\Lambda} \times \boldsymbol{r}_{\mathrm{N}}\right|$. The $r$-dependent coefficients $V_{\alpha}(r)$ of the terms above (Eqn. 1.2) can be parametrized in a three-range Gaussian form [11], [12] as

$$
V_{\alpha}(r)=\sum_{i}\left(a_{i}+b_{i} k_{F}+c_{i} k_{F}^{2}\right) \exp \left[-\left(r / \beta_{i}\right)^{2}\right]
$$

where parameters $a_{i}, b_{i}$ and $c_{i}$ are given for each $\alpha$, i.e. spin-spin $(\sigma), \Lambda$ spin-orbit $(\Lambda)$, induced nucleon spin-orbit $(\mathrm{N})$ and tensor $(T)$ terms in Eqn. 1.2. $k_{F}$ is the Fermi momentum. A wide variety of hypernuclear properties such as level structure and reaction cross sections are calculated using this potential and compared directly with experimental data.

\subsection{Historical overview}

Investigation of $\Lambda$ hypernuclei has been developed through three generations since the first observation of the $\Lambda$ particle which was demonstrated by an air bone emulsion experiment late in 1952 by Danysz et al. [13]. Fig. 1.3 shows the first hypernuclear tracks of particles in a photographic emulsion. A cosmic ray coming in from the top right (marked p) collided with a nucleus in the emulsion. One of the fragments from the collision decayed at the vertex marked as B to produce three new tracks. The faintest of these, travelling towards the lower left (and marked 3 on the original), is probably due to a pion. The total energy released in the disintegration is consistent with the decay of a lambda particle in the original nuclear fragment.

In the first generation, the studies of $\Lambda$ hypernuclei with $\mathrm{K}^{-}$beams were carried out with emulsion techniques at CERN and BNL. During this era, light $\Lambda$ hypernuclei $(A \leq 16)$ were observed through their weak decay process, and the binding energies of their ground states were determined with kinematic constraints [14]. As a result, the depth of the $\Lambda$-nucleus potential was found to be $\sim 2 / 3$ of that in the nucleon case. However, experimental data were limited to the ground states of light $\Lambda$ hypernuclei, and excited states could not be investigated fully.

For the second generation, counter experiments started at CERN and later at BNL using 


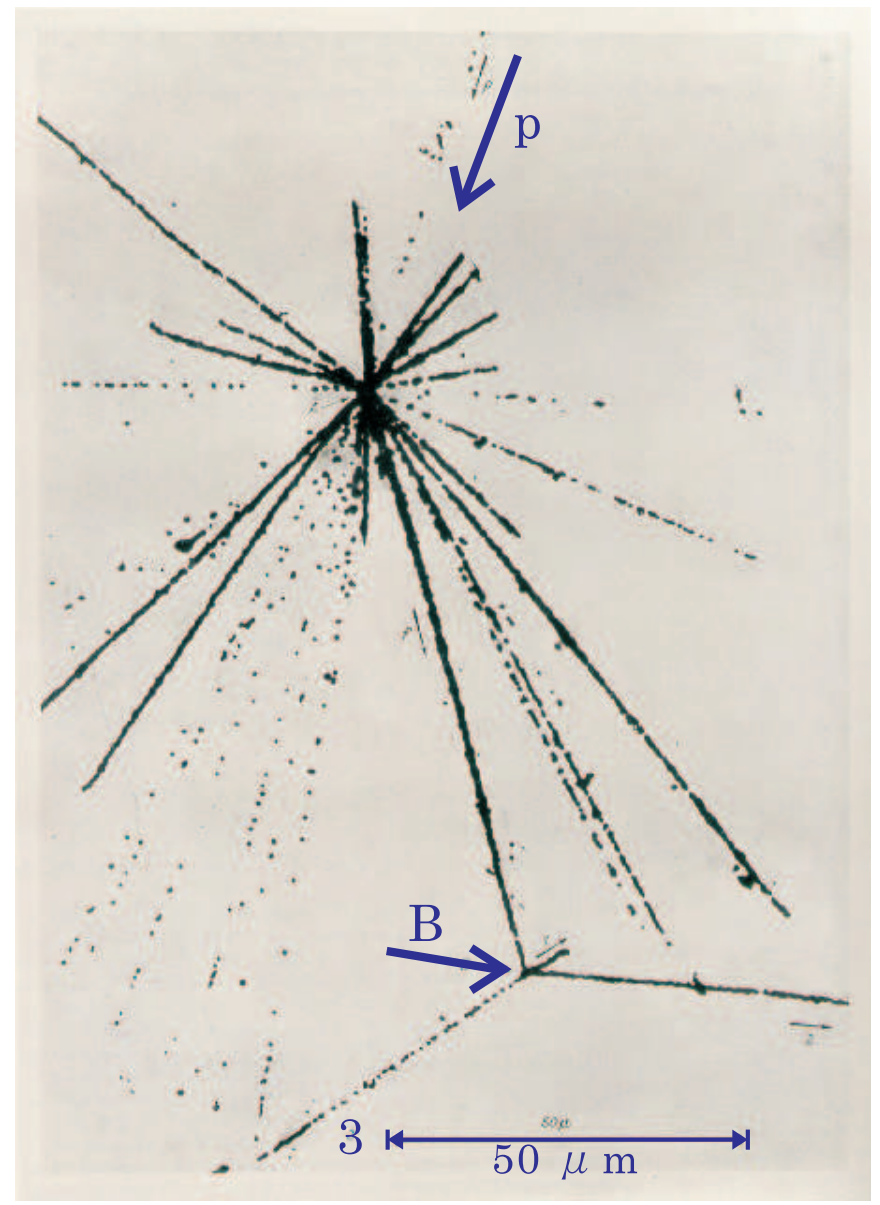

Figure 1.3: The first observation of a $\Lambda$ hypernucleus in a photographic emulsion by Danysz et al. [13]. 
$\mathrm{K}^{-}$beams in the early 1970s, and spectroscopic studies also including excited states came to be realized.

At the beginning of the second generation, the $\gamma$-ray transitions from the excited states of ${ }_{\Lambda}^{4} \mathrm{H}$ and ${ }_{\Lambda}^{4} \mathrm{He}$, populated through the $\left(\mathrm{K}_{\text {stop }}^{-}, \pi^{-}\right)$reaction, were measured at CERN in 1971 [15]. Then in 1973, the first spectroscopic experiment with a magnetic spectrometer was carried out for the ${ }^{12} \mathrm{C}\left(\mathrm{K}_{\text {stop }}^{-}, \pi^{-}\right)_{\Lambda}^{12} \mathrm{C}$ reaction in CERN [16], and followed by a novel method of the inflight $\left(\mathrm{K}^{-}, \pi^{-}\right)$reaction in an almost recoilless condition for various $\Lambda$ hypernuclei up to mass number of 209 at CERN [17-22] and BNL [23,24] in 1970s and 1980s. In particular, p-shell hypernuclei were intensively investigated and it was found that the spin-orbit splittings of $\Lambda$ orbits are quite small $[19,20]$. The spectroscopy with the in-flight $\left(\mathrm{K}^{-}, \pi^{-}\right)$reaction brought significant achievements, particularly for the studies of light $p$-shell $\Lambda$ hypernuclei compared to the experiments with the emulsion techniques and $\left(\mathrm{K}_{\text {stop }}^{-}, \pi^{-}\right)$reaction. However, data quality itself was not sufficient in terms of statistics and resolution due to low $\mathrm{K}^{-}$beam intensity, sizable emittance and difficulties of beam momentum identification.

In the third generation, the $\left(\pi^{+}, \mathrm{K}^{+}\right)$reaction took over as an effective tool for the $\Lambda$ spectroscopic studies of heavier hypernuclei up to the range of $A=89$ since mid-1980s at the AGS (BNL) [25, 26] and $12 \mathrm{GeV}$ PS (KEK) [27]. As discussed in Chap. 1.4, the endothermic, $\left(\pi^{+}, \mathrm{K}^{+}\right)$reaction has a large recoil momentum, thus deeply bound states can be excited even for heavier $\Lambda$ hypernuclei and moreover, the highest spin states with maximum aligned angular momentum. After the Superconducting Kaon Spectrometer (SKS) at the KEK $12 \mathrm{GeV}$ PS became available for experiments, hypernuclear mass spectra up to a mass number of 208 were measured with an energy resolution of 1.45-2.0 MeV (FWHM) and good statistics [28-31]. Deeply bound states, such as $s_{\Lambda}$ and $p_{\Lambda}$, were identified in a wide range of spectra. Particularly, fine peak structures related to core-excited states were observed for light hypernuclei of ${ }_{\Lambda}^{10} \mathrm{~B}$ and ${ }_{\Lambda}^{12} \mathrm{C}$ and provided various inputs constraints to understand the $\Lambda \mathrm{N}$ interaction by precise comparisons with configuration mixing shell model calculations [5, 9, 32,33]. For the heavier hypernuclei, ${ }_{\Lambda}^{89} \mathrm{Y},{ }_{\Lambda}^{139} \mathrm{La}$ and ${ }_{\Lambda}^{208} \mathrm{~Pb}$, peak structures reflecting $\Lambda$ shell structure were obtained.

Furthermore, the $\Lambda$ binding energies were measured over a wide mass range and the mass number dependence of the obtained binding energies was found to be consistent with the independent single particle picture of $\Lambda$ hypernuclei [29].

And finally, in the last decade, two experimental techniques have been developed which are symbolized by the introduction of the $\gamma$-ray spectroscopy on the hadronic hypernuclei production reaction, and of the hypernuclear spectroscopy with the $\left(\mathrm{e}, \mathrm{e}^{\prime} \mathrm{K}^{+}\right)$reaction.

As introduced above, the $\gamma$-ray spectroscopic technique itself has already been utilized for hypernuclear spectroscopy since the 1970s in CERN [15] using NaI crystals. In the 1980s, the first observation of $\gamma$-ray transitions on $p$-shell $\Lambda$ hypernuclei were successfully conducted with a NaI detector at the BNL-AGS [34,35]. Germanium (Ge) detectors were used for the first time for the $\gamma$-ray hypernuclear spectroscopy at the KEK $12 \mathrm{GeV}$ PS in 1998 with an unprecedented energy resolution of a few $\mathrm{keV}$, and the $\gamma$-ray transitions $\left(3 / 2^{+} \rightarrow 1 / 2^{+}, 5 / 2^{+} \rightarrow 1 / 2^{+}\right.$, etc.) of ${ }_{\Lambda}^{7} \mathrm{Li}$ have been observed with a combination of Ge and BGO systems (Hyperball) [36, 37].

After that, the $\gamma$-ray spectroscopic studies for the $p$-shell $\Lambda$ hypernuclei with the Hyperball were performed not only at KEK [38-41], but also at BNL [42-44]. The experimental data from these precise $\gamma$-ray spectroscopies provided crucial information on the strength of the spin-spin interaction between a $\Lambda$ and a nucleon and indicated that the size of the core nucleus in a $\Lambda$ hypernucleus is smaller than that of an ordinary nucleus.

Another development is the introduction of the $\left(\mathrm{e}, \mathrm{e}^{\prime} \mathrm{K}^{+}\right)$reaction to the hypernuclear spectroscopy.

With the JLab-CEBAF completion in 1996, the high resolution spectroscopic study of the $\Lambda$ hypernuclei through the electro-photoproduction reaction became possible with the high in- 
tensity and high quality continuous electron beam. The first experiment of $\Lambda$ hypernuclear spectroscopy with the $\left(\mathrm{e}, \mathrm{e}^{\prime} \mathrm{K}^{+}\right)$reaction was successfully demonstrated for ${ }_{\Lambda}^{12} \mathrm{~B}$ in 2000 , and observed characteristic $\Lambda$ single particle states corresponding to $s_{\Lambda}$ and $p_{\Lambda}$ orbits, as well as a few core excited states with sub MeV (FWHM) resolution for ${ }_{\Lambda}^{12} \mathrm{~B}[45,46]$.

By combining precise spin doublet splitting information with $\gamma$-ray spectroscopy and absolute level scheme measurement with $\left(\mathrm{e}, \mathrm{e}^{\prime} \mathrm{K}^{+}\right)$reaction spectroscopy, understanding of the interior structure of $\Lambda$ hypernuclei is expected to progress.

The $\Lambda$ hypernuclei which have been identified experimentally so far are summarized in Fig. 1.4.

\section{$\Lambda$ Hypernuclear Chart}

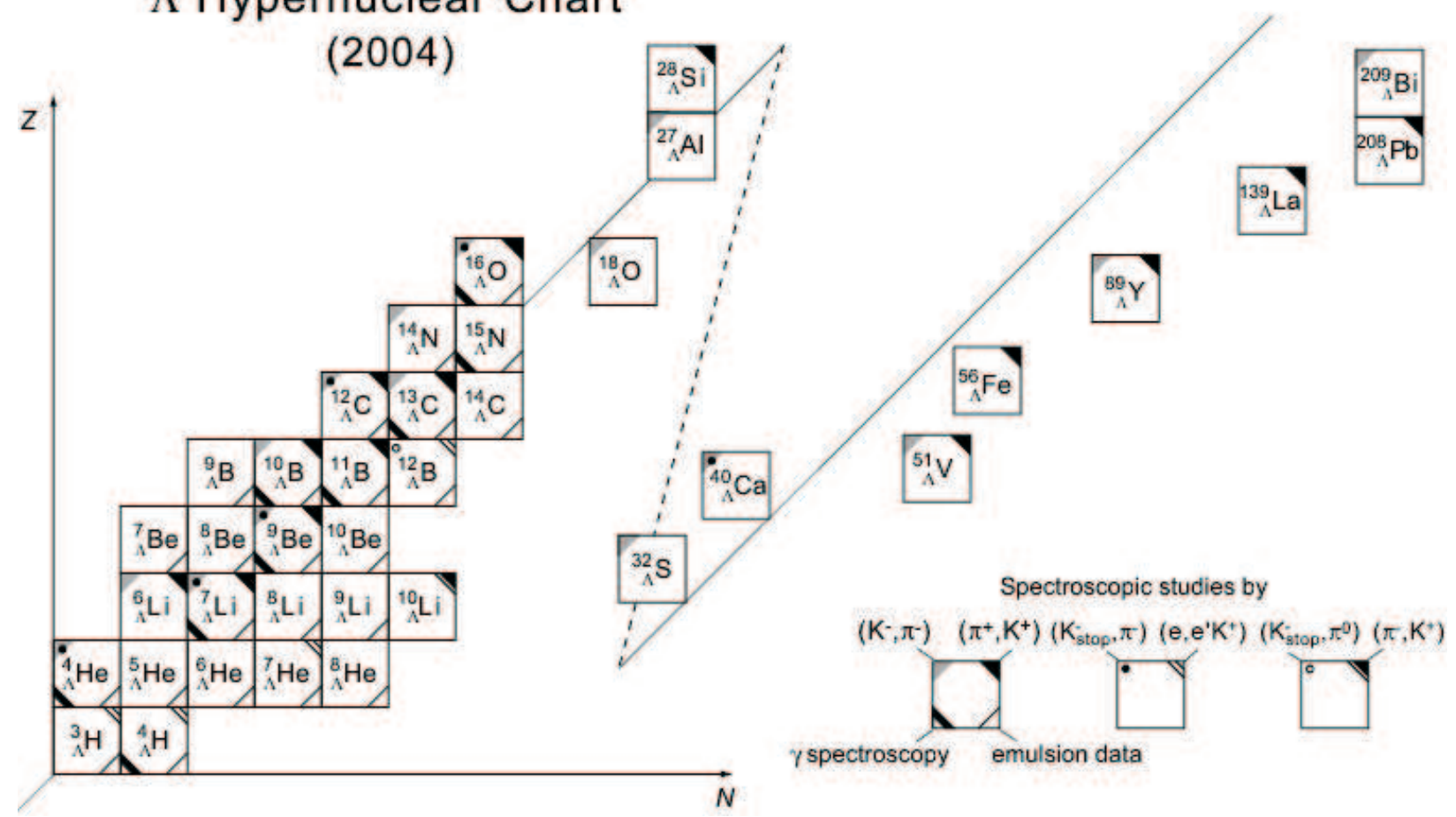

Figure 1.4: $\Lambda$ hypernuclei which have been observed by emulsion techniques, reaction spectroscopy of $\left(\mathrm{K}^{-}, \pi^{-}\right),\left(\pi^{+}, \mathrm{K}^{+}\right),\left(\mathrm{e}, \mathrm{e}^{\prime} \mathrm{K}^{+}\right)$and $\gamma$-ray spectroscopy [47].

\subsection{Hypernuclear production mechanisms}

A $\Lambda$ hypernucleus can be produced by the strange quark exchange $\left(\mathrm{K}^{-}, \pi^{-}\right)$reaction and strange quark pair production $\left(\pi^{+}, \mathrm{K}^{+}\right)$or $\left(\gamma, \mathrm{K}^{+}\right),\left(\mathrm{e}, \mathrm{e}^{\prime} \mathrm{K}^{+}\right)$reactions as shown in Fig. 1.5. In the $\left(\mathrm{K}^{-}, \pi^{-}\right)$and $\left(\pi^{+}, \mathrm{K}^{+}\right)$reactions, a neutron in the target nucleus ${ }^{A} Z$ is converted into a $\Lambda$. The $\Lambda$ is coupled to the nuclear core to form a $\Lambda$ hypernucleus ${ }_{\Lambda}^{A} Z$. On the other hand, electro and photo-production reactions $\left(\gamma, \mathrm{K}^{+}\right)$and $\left(\mathrm{e}, \mathrm{e}^{\prime} \mathrm{K}^{+}\right)$convert a proton in a nucleus ${ }^{A} Z$ to a $\Lambda$ and form hypernucleus ${ }_{\Lambda}^{A}(Z-1)$.

Hypernuclear production cross section depends mainly on reaction processes and momentum transfer to the $\Lambda$.

The cross section of the strangeness exchange reaction of $\left(\mathrm{K}^{-}, \pi^{-}\right)$is known to be about $4 \mathrm{mb} / \mathrm{sr}$ near $p_{\mathrm{K}^{-}}=800 \mathrm{MeV} / c$ at forward angles [10]. It is estimated to be one order of magnitude larger than the strangeness production of the $\left(\pi^{+}, \mathrm{K}^{+}\right)$reaction which has a maximum cross 


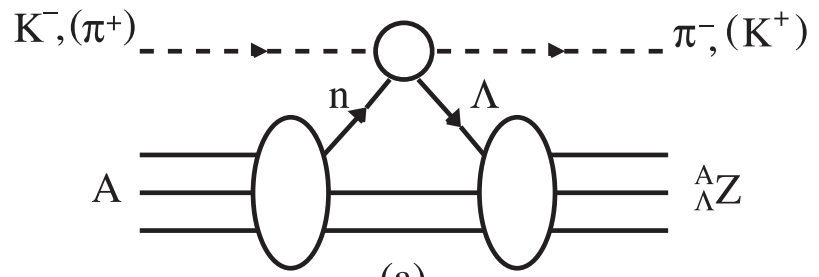

(a)

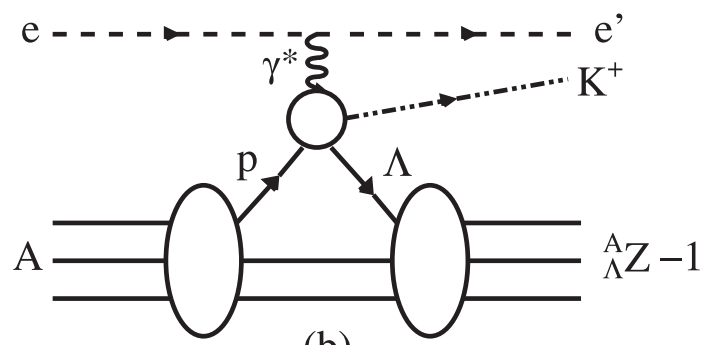

(b)

Figure 1.5: Schematic of the hypernuclear (a): hadronic production and (b): electroproduction.

section of about $280 \mu \mathrm{b} / \mathrm{sr}$ near $p_{\pi^{+}}=1.05 \mathrm{GeV} / c$ at forward angles [48].

On the other hand, the photoproduction process has a much smaller cross section of about 0.3 $\mu \mathrm{b} / \mathrm{sr}$, three orders of magnitude less than that of the $\left(\pi^{+}, \mathrm{K}^{+}\right)$reaction [49].

However, this smaller cross section can be partially recovered by the high intensity of electron beams which is provided by the Continuous Electron Beam Accelerator Facility (CEBAF) at the Thomas Jefferson National Accelerator Facility (JLab).

Another factor which controls hypernuclear production rates is the hyperon recoil momentum, in other words, the momentum transfer to the $\Lambda$ particle. Fig. 1.6 shows the hyperon recoil momentum as a function of projectile momentum for different hyperon production reactions at detection angle of $\theta_{l a b}=0^{\circ}$. Let us focus only on the process which is associated with $\Lambda$ production. In order to produce a hypernucleus, the hyperon emerging through the reaction

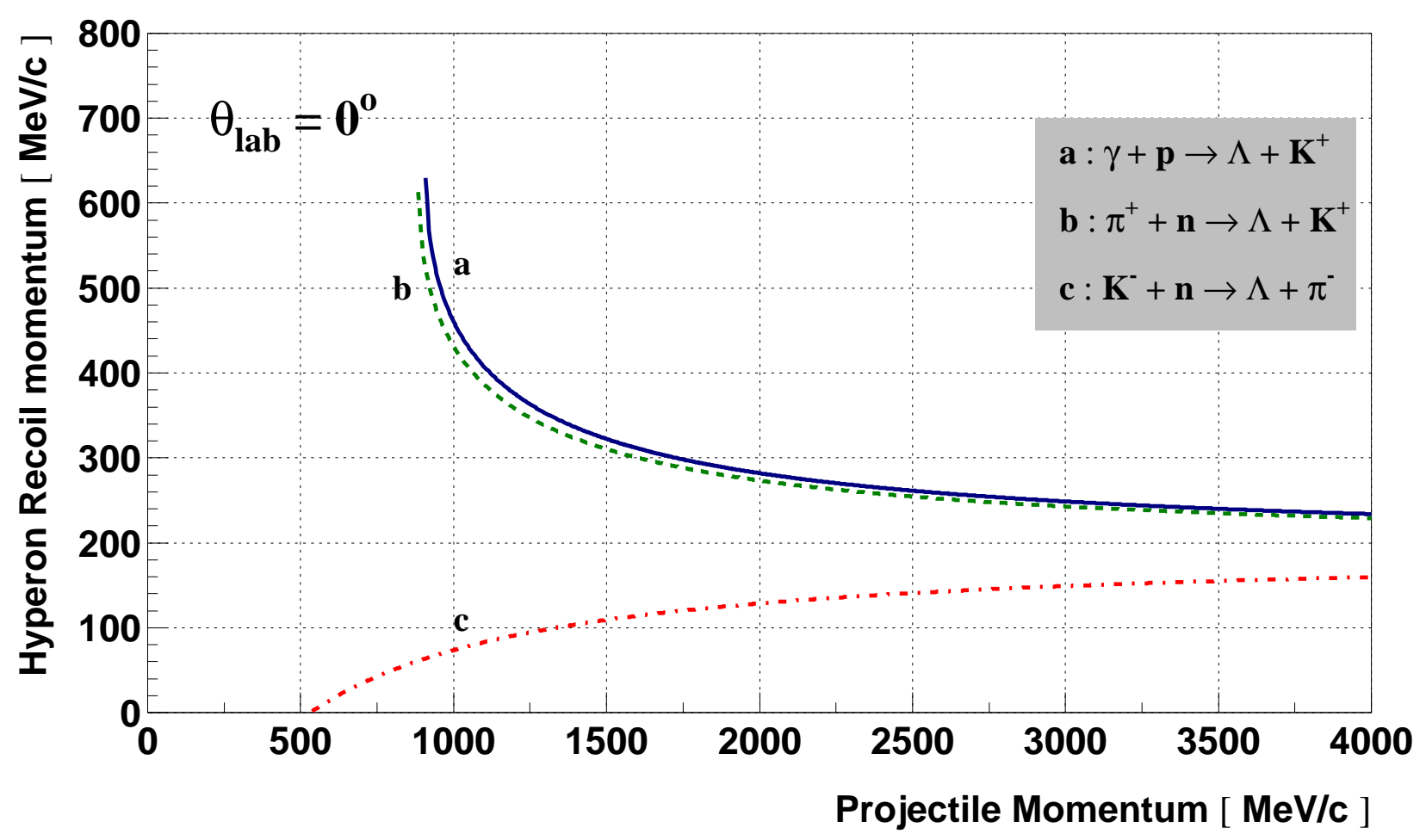

Figure 1.6: Hyperon recoil momenta $\left(\theta=0^{\circ}\right)$ as a function of projectile momentum with lab frame for the $\left(\gamma, \mathrm{K}^{+}\right),\left(\pi^{+}, \mathrm{K}^{+}\right)$and $\left(\mathrm{K}^{-}, \pi^{-}\right)$reaction [50].

on a nucleon in the target nucleus has to stay bound to the nucleus. The probability of this phenomenon, the so called "sticking probability", depends very much on the momentum transfer to the hyperon. If the momentum transfer is too large compared to the nuclear Fermi 
momentum $(\sim 270 \mathrm{MeV} / c)$, the hyperon will leave the nucleus and the sticking probability will be small. If the momentum transfer is very small, the hyperon will have a chance to stay in the nucleus and will most probably keep the orbit of the original nucleon [50].

As shown in Fig. 1.6, the exothermic reaction of $\left(\mathrm{K}^{-}, \pi^{-}\right)$has a magic momentum $p_{\mathrm{K}^{-}} \sim 0.55$ $\mathrm{GeV} / c$ for the $\Lambda$ production process, where the momentum transfer vanishes and a recoilless $\Lambda$ production takes place.

In the case of the endothermic reactions $\left(\gamma, \mathrm{K}^{+}\right)$and $\left(\pi^{+}, \mathrm{K}^{+}\right)$, the momentum transfer is maximal at the thresholds and approaches an asymptotic value, as the projectile momenta increase.

The produced states of the hypernuclei strongly depend on the hyperon recoil momentum. In the case of the $\left(\mathrm{K}^{-}, \pi^{-}\right)$reaction, due to the recoilless or low momentum transfer, the orbital angular momentum transfer $\Delta L=0$ and spin transfer $\Delta J=0$ are favored. In other words, a $\Lambda$ particle tends to populate an energy level which contains the same orbital angular momentum as a neutron in the outermost shell. For example, in the case of the ${ }^{12} \mathrm{C}\left(\mathrm{K}^{-}, \pi^{-}\right){ }_{\Lambda}^{12} \mathrm{C}$ reaction, the neutron in the $p_{3 / 2}$ orbit is easily replaced with the $p_{3 / 2} \Lambda$.

On the other hand, the $\left(\pi^{+}, \mathrm{K}^{+}\right)$process will favorably excite high-spin stretched configuration of a hypernucleus with maximum aligned angular momentum : $J=L_{\max }=l_{n}+l_{\Lambda}(l$ is the orbital angular momentum) for the ground state $0^{+}$to $J^{\pi}\left[(l j)_{n}^{-1}(l j)_{\Lambda}\right]$ state transition. For example, in the ${ }^{12} \mathrm{C}\left(\pi^{+}, \mathrm{K}^{+}\right){ }_{\Lambda}^{12} \mathrm{C}$ reaction, the probability to replace a $s_{1 / 2} \Lambda$ with a $p_{3 / 2}$ neutron is also increased. Therefore, the $\left(\pi^{+}, \mathrm{K}^{+}\right)$reaction is more suitable for the investigation of deeply bound $\Lambda$ hypernuclei than the $\left(\mathrm{K}^{-}, \pi^{-}\right)$reaction.

The photoproduction $\left(\gamma, \mathrm{K}^{+}\right)$reaction also features large momentum transfer as does the $\left(\pi^{+}\right.$, $\left.\mathrm{K}^{+}\right)$reaction. The threshold photon energy for ${ }^{12} \mathrm{C}\left(\gamma, \mathrm{K}^{+}\right)$at $0^{\circ}$ detection is $687 \mathrm{MeV} / c$, and the typical momentum transfer for $\Lambda$ production through the $\left(\gamma, \mathrm{K}^{+}\right)$reaction is about $250 \sim$ $300 \mathrm{MeV} / c$ as shown in Fig. 1.6 at forward angle. The $\left(\gamma, \mathrm{K}^{+}\right)$reaction also favors excitation of high spin states, however unlike the pseudo-scalar mesons of pions and kaons, photons carry spin $J=1$, so the spin-flip state will be also excited as shown in Fig. 2.5.

\subsection{Electroproduction of hypernuclei}

\subsubsection{Elementary amplitude and kinematics}

The kinematics of the electroproduction reaction (elementary process),

$$
e\left(p_{\mathrm{e}}\right)+\mathrm{p}\left(p_{\mathrm{p}}\right) \rightarrow e\left(p_{\mathrm{e}^{\prime}}\right)+\Lambda\left(p_{\Lambda}\right)+\mathrm{K}^{+}\left(p_{\mathrm{K}^{+}}\right)
$$

is described in Fig. 1.7. The variables in the parentheses in Eqn. 1.5 represents the fourmomentum of each particle.

The momentum and energy of the virtual photon are defined as $\boldsymbol{q}=\boldsymbol{p}_{\mathrm{e}}-\boldsymbol{p}_{\mathrm{e}}$, and $\omega=E_{\mathrm{e}}-E_{\mathrm{e}}$, respectively. An azimuthal angle $\Phi_{\mathrm{K}^{+}}$defines the angle between the scattering and reaction planes.

The triple-differential cross section is described as [51]

$$
\begin{aligned}
\frac{d^{3} \sigma}{d E_{\mathrm{e}}, d \Omega_{\mathrm{e}}, d \Omega_{\mathrm{K}^{+}}}= & \frac{1}{\left|\boldsymbol{v}_{\mathrm{e}}-\boldsymbol{v}_{\mathrm{p}}\right|} \frac{\alpha^{2}}{Q^{2}} \frac{E_{\mathrm{e}}}{E_{\mathrm{e}}} \frac{1}{1-\epsilon}\left\{\left(W^{x x}+W^{y y}\right)+\epsilon\left(W^{x x}-W^{y y}\right)\right. \\
& \left.+2 \epsilon \frac{Q^{2}}{\omega^{2}} W^{z z}-\sqrt{2 \epsilon(1+\epsilon) \frac{Q^{2}}{\omega^{2}}}\left(W^{z x}+W^{x z}\right)\right\},
\end{aligned}
$$




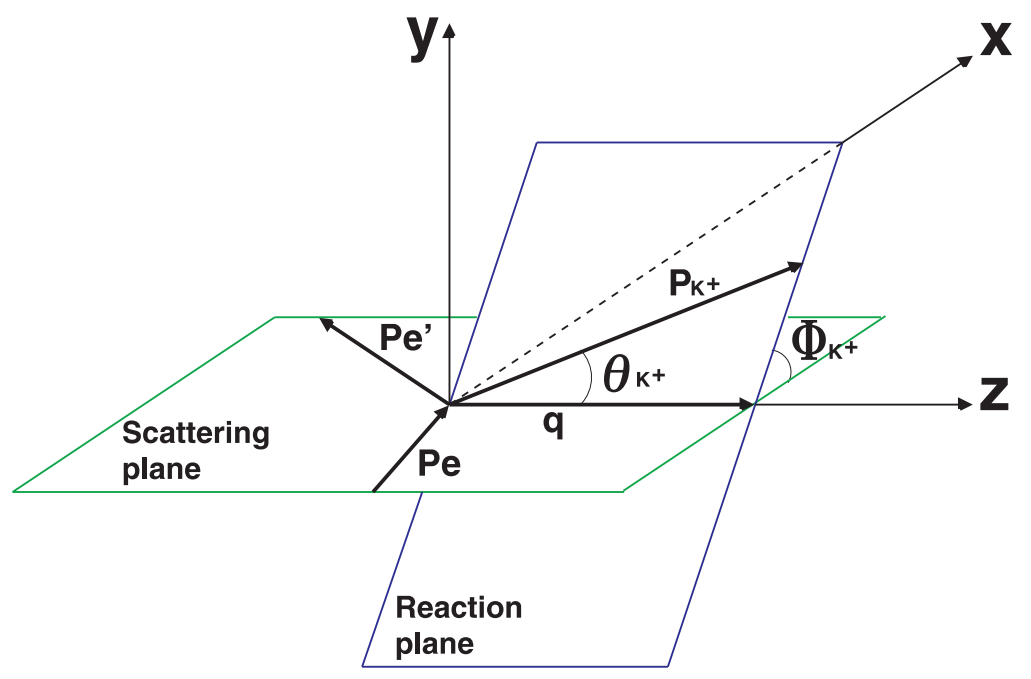

Figure 1.7: Kinematics of electroproduction. The initial $\left(p_{\mathrm{e}}\right)$ and final $\left(p_{\mathrm{e}}\right)$ electron momenta define the scattering plane $x z$. The transferred momentum $q=p_{\mathrm{e}}-p_{\mathrm{e}}$, is directed along the $z$-axis, and the momentum of the kaon $p_{\mathrm{K}^{+}}$defines the reaction plane.

where $\alpha=e^{2} / 4 \pi=1 / 137, Q^{2}=-q^{2}>0$ and

$$
\epsilon=\left(1+\frac{2|\boldsymbol{q}|^{2}}{Q^{2}} \tan ^{2} \frac{\theta_{\mathrm{e}}}{2}\right)^{-1}
$$

with an electron scattering angle of $\theta_{\mathrm{e}}$.

The hadron tensor $W^{\mu \nu}$ is described by :

$$
\begin{aligned}
W^{\mu \nu}= & \frac{1}{(2 \pi)^{3}} \frac{m_{\mathrm{p}} m_{\Lambda}}{E_{\mathrm{p}} E_{\Lambda}} \int d\left|\boldsymbol{p}_{\mathrm{K}^{+}}\right| \frac{\left|\boldsymbol{p}_{\mathrm{K}^{+}}\right|^{2}}{2 E_{\mathrm{K}^{+}}} d \boldsymbol{p}_{\Lambda} \delta^{4}\left(p_{\Lambda}+p_{\mathrm{K}^{+}}-p_{\mathrm{p}}-q\right) \\
& \times \frac{1}{2} \sum_{\text {spin }} J^{\mu}\left(p_{\mathrm{K}^{+}} p_{\Lambda}, p_{\mathrm{p}} q\right) J^{\nu^{+}}\left(p_{\mathrm{K}^{+}} p_{\Lambda}, p_{\mathrm{p}} q\right) .
\end{aligned}
$$

$J^{\mu}\left(p_{\mathrm{K}^{+}} p_{\Lambda}, p_{\mathrm{p}} q\right)$ is the hadron current matrix which is explained below.

Performing the integration in Eqn. 1.8,

$$
\begin{aligned}
W^{\mu \nu}= & \frac{1}{(2 \pi)^{3}} \frac{m_{\mathrm{p}} m_{\Lambda}}{E_{\mathrm{p}} E_{\Lambda}} \frac{\left|\boldsymbol{p}_{\mathrm{K}^{+}}\right|^{2}}{4 E_{\mathrm{K}^{+}} R} \sum_{\text {spin }} J^{\mu}\left(p_{\mathrm{K}^{+}} p_{\Lambda}, p_{\mathrm{p}} q\right) J^{\nu^{+}}\left(p_{\mathrm{K}^{+}} p_{\Lambda}, p_{\mathrm{p}} q\right) \\
\equiv & \frac{1}{(2 \pi)^{3}} \frac{m_{\mathrm{p}} m_{\Lambda}}{E_{\mathrm{p}} E_{\Lambda}} \frac{\left|\boldsymbol{p}_{\mathrm{K}^{+}}\right|^{2}}{4 E_{\mathrm{K}^{+}} R} \tilde{W}^{\mu \nu} \\
& R=\frac{\left|\boldsymbol{p}_{\mathrm{K}^{+}}\right|}{E_{\mathrm{K}^{+}}}-\frac{\boldsymbol{p}_{\mathrm{K}^{+}} \cdot \boldsymbol{p}_{\Lambda}}{p_{\mathrm{K}^{+}} E_{\Lambda}},
\end{aligned}
$$

where $\boldsymbol{p}_{\Lambda}$ and $\left|\mathrm{p}_{\mathrm{K}^{+}}\right|$are fixed by the energy and momenta conservation. Finally, putting $\mid \mathrm{v}_{\mathrm{e}}-$ $\mathrm{v}_{\mathrm{p}} \mid=\left(\boldsymbol{p}_{\mathrm{e}} \cdot \boldsymbol{p}_{\mathrm{p}}\right) /\left(E_{\mathrm{e}} E_{\mathrm{p}}\right)\left(\right.$ for $\left.m_{\mathrm{e}}=0\right)$, the cross section Eqn. 1.6 is rewritten as : 


$$
\begin{aligned}
\frac{d^{3} \sigma}{d E_{\mathrm{e}}, d \Omega_{\mathrm{e}}, d \Omega_{\mathrm{K}^{+}}}= & \frac{1}{(2 \pi)^{3}} \frac{\alpha^{2}}{Q^{2}(1-\epsilon)} \frac{E_{\mathrm{e}} \cdot m_{\mathrm{p}}}{\left(\boldsymbol{p}_{\mathrm{e}} \cdot \boldsymbol{p}_{\mathrm{p}}\right)} \frac{m_{\Lambda}\left|\boldsymbol{p}_{\mathrm{K}^{+}}\right|^{2}}{4 E_{\Lambda} E_{\mathrm{K}^{+}} R}\left\{\left(\tilde{W}^{x x}+\tilde{W}^{y y}\right)\right. \\
& \left.+\epsilon\left(\tilde{W}^{x x}-\tilde{W}^{y y}\right)+2 \epsilon \frac{Q^{2}}{\omega^{2}} \tilde{W}^{z z}-\sqrt{2 \epsilon(1+\epsilon) \frac{Q^{2}}{\omega^{2}}}\left(\tilde{W}^{z x}+\tilde{W}^{x z}\right)\right\}
\end{aligned}
$$

For the elementary process, it is common practice to evaluate the electron kinematics in the laboratory frame but to express the cross section in terms of the meson and variables in their center-of-mass (CMS) frame.

In order to express the cross section Eqn. 1.10 in the form that allows a connection to the photoproduction (by real photons), the effective photon energy $E_{\gamma}$ and the virtual photon flux $\Gamma$, which is commonly evaluated using the lab frame, are defined as :

$$
\begin{gathered}
E_{\gamma}=\omega-\frac{q^{2}}{2 m_{\mathrm{p}}}, \\
\Gamma=\frac{\alpha}{2 \pi^{2} Q^{2}} \frac{1}{1-\epsilon} \frac{E_{\mathrm{e}} \cdot m_{\mathrm{p}}}{\left(\boldsymbol{p}_{\mathrm{e}} \cdot \mathrm{p}_{\mathrm{p}}\right)} E_{\gamma} \stackrel{\text { lab }}{=} \frac{\alpha}{2 \pi^{2} Q^{2}} \frac{E_{\gamma}}{1-\epsilon} \frac{E_{\mathrm{e}}}{E_{\mathrm{e}}} .
\end{gathered}
$$

The effective photon energy $E_{\gamma}$ represents the energy of the real photon that would yield (in the lab frame) as the same as the actual virtual photon in electroproduction.

Then separating the $\Phi_{\mathrm{K}^{+}}$dependent part and $\Gamma$, the triple-differential cross section is written as [52] :

$$
\frac{d^{3} \sigma}{d E_{\mathrm{e}^{\prime}} d \Omega_{\mathrm{e}^{\prime}} d \Omega_{\mathrm{K}^{+}}}=\Gamma\left\{\frac{d \sigma_{\mathrm{T}}}{d \Omega_{\mathrm{K}^{+}}}+\epsilon \frac{d \sigma_{\mathrm{L}}}{d \Omega_{\mathrm{K}^{+}}}+\epsilon \frac{d \sigma_{\mathrm{p}}}{d \Omega_{\mathrm{K}^{+}}}+\sqrt{2 \epsilon(1+\epsilon)} \frac{d \sigma_{\mathrm{I}}}{d \Omega_{\mathrm{K}^{+}}} \cos 2 \Phi_{\mathrm{K}^{+}}\right\},
$$

where $\sigma_{\mathrm{T}}, \sigma_{\mathrm{L}}, \sigma_{\mathrm{P}}$ and $\sigma_{\mathrm{I}}$ are called transverse, longitudinal, polarization and interference cross section, respectively. They are given in terms of the baryon currents as follows :

$$
\begin{aligned}
\frac{d \sigma_{\mathrm{T}}}{d \Omega_{\mathrm{K}^{+}}} & =\frac{\alpha}{4 \pi} \frac{m_{\Lambda}}{E_{\Lambda}} \frac{\left|\boldsymbol{p}_{\mathrm{K}^{+}}\right|^{2}}{4 E_{\mathrm{K}^{+}} R} \frac{1}{E_{\gamma}}\left(\tilde{W}^{x x}+\tilde{W}^{y y}\right)_{\Phi_{\mathrm{K}^{+}}=0}, \\
\frac{d \sigma_{\mathrm{L}}}{d \Omega_{\mathrm{K}^{+}}} & =\frac{\alpha}{4 \pi} \frac{m_{\Lambda}}{E_{\Lambda}} \frac{\left|\boldsymbol{p}_{\mathrm{K}^{+}}\right|^{2}}{4 E_{\mathrm{K}^{+}} R} \frac{1}{E_{\gamma}} \frac{2 Q^{2}}{\omega^{2}}\left(\tilde{W}^{z z}\right)_{\Phi_{\mathrm{K}^{+}}=0}, \\
\frac{d \sigma_{\mathrm{P}}}{d \Omega_{\mathrm{K}^{+}}} & =\frac{\alpha}{4 \pi} \frac{m_{\Lambda}}{E_{\Lambda}} \frac{\left|\boldsymbol{p}_{\mathrm{K}^{+}}\right|^{2}}{4 E_{\mathrm{K}^{+}} R} \frac{1}{E_{\gamma}}\left(\tilde{W}^{x x}-\tilde{W}^{y y}\right)_{\Phi_{\mathrm{K}^{+}}=0}, \\
\frac{d \sigma_{\mathrm{I}}}{d \Omega_{\mathrm{K}^{+}}} & =\frac{\alpha}{4 \pi} \frac{m_{\Lambda}}{E_{\Lambda}} \frac{\left|\boldsymbol{p}_{\mathrm{K}^{+}}\right|^{2}}{4 E_{\mathrm{K}^{+}} R} \frac{1}{E_{\gamma}} \sqrt{\frac{Q^{2}}{\omega^{2}}}\left(\tilde{W}^{z x}+\tilde{W}^{x z}\right)_{\Phi_{\mathrm{K}^{+}}=0} .
\end{aligned}
$$

The hadron current matrix element which is appeared in Eqn. 1.8 is decomposed into six gauge invariant terms :

$$
\begin{aligned}
J^{\mu}\left(p_{\mathrm{K}^{+}} p_{\Lambda}, p_{\mathrm{p}} q\right) & =\sum_{i} A_{i} \bar{u}\left(p_{\Lambda}\right) M_{i}^{\mu} u\left(p_{\mathrm{p}}\right) \\
M_{1}^{\mu} & =\frac{1}{2} \gamma_{5}\left\{(\gamma \cdot q) \gamma^{\mu}-\gamma^{\mu}(\gamma \cdot q)\right\} \\
M_{2}^{\mu} & =\gamma_{5}\left\{q^{2} p_{\mathrm{p}}^{\mu}-\left(q \cdot p_{\mathrm{p}}\right) q^{\mu}\right\}
\end{aligned}
$$




$$
\begin{aligned}
M_{3}^{\mu} & =\gamma_{5}\left\{q^{2} p_{\Lambda}^{\mu}-\left(q \cdot p_{\Lambda}\right) q^{\mu}\right\} \\
M_{4}^{\mu} & =\gamma_{5}\left\{\gamma^{\mu}\left(q \cdot p_{\mathrm{p}}\right)-(\gamma \cdot q) p_{\mathrm{p}}^{\mu}\right\} \\
M_{5}^{\mu} & =\gamma_{5}\left\{\gamma^{\mu}\left(q \cdot p_{\Lambda}\right)-(\gamma \cdot q) p_{\Lambda}^{\mu}\right\} \\
M_{6}^{\mu} & =\frac{1}{2} \gamma_{5}\left\{(\gamma \cdot q) q^{\mu}-\gamma^{\mu} q^{2}\right\}
\end{aligned}
$$

The detail description of the scalar form factors $A_{i}$ are explained in [51].

Although it is possible to calculate the hadronic tensor $\tilde{W}^{i j}$ directly from Eqn. 1.9 and 1.15, it is more convenient and common practice to go over to a two-component formalism. Eventually, the elementary transition matrix of the virtual photon induced reaction for the elementary process is written in the CMS frame $\left(\boldsymbol{p}_{\mathrm{p}}=-\boldsymbol{q}, \boldsymbol{p}_{\Lambda}=-\boldsymbol{p}_{\mathrm{K}^{+}}\right)$:

$$
\begin{aligned}
\boldsymbol{\epsilon} \cdot \boldsymbol{J}\left(p_{\mathrm{K}^{+}} p_{\Lambda}, p_{\mathrm{p}} q\right)= & f_{1}(\boldsymbol{\sigma} \cdot \boldsymbol{\epsilon})+i f_{2}\left(\boldsymbol{\sigma} \cdot \hat{p}_{\mathrm{K}^{+}}\right)\{\boldsymbol{\sigma} \cdot(\hat{q} \times \boldsymbol{\epsilon})\}+f_{3}(\boldsymbol{\sigma} \cdot \hat{q})\left(\hat{p}_{\mathrm{K}^{+}} \cdot \boldsymbol{\epsilon}\right) \\
& +f_{4}\left(\boldsymbol{\sigma} \cdot \hat{p}_{\mathrm{K}^{+}}\right)\left(\hat{p}_{\mathrm{K}^{+}} \cdot \boldsymbol{\epsilon}\right)+f_{5}(\boldsymbol{\sigma} \cdot \hat{q})(\hat{q} \cdot \boldsymbol{\epsilon})+f_{6}\left(\boldsymbol{\sigma} \cdot \hat{p}_{\mathrm{K}^{+}}\right)(\hat{q} \cdot \boldsymbol{\epsilon})
\end{aligned}
$$

where $\hat{q}=\boldsymbol{q} /|\boldsymbol{q}|, \hat{p}_{\mathrm{K}^{+}}=\boldsymbol{p}_{\mathrm{K}^{+}} /\left|\boldsymbol{p}_{\mathrm{K}^{+}}\right|, \boldsymbol{\sigma}$ are the Pauli matrices, $\boldsymbol{\epsilon}$ is the polarization vector of the virtual photon. The functions $f_{i}(i=1 \sim 6)$ are expressed in terms of the form factors $A_{i}$, which are explained in Appendix [51].

\subsubsection{Electroproduction of hypernuclei}

Next, these above formulae for the elementary process are applied to the electroproduction of hypernuclei in nuclear media :

$$
\mathrm{e}+\mathrm{A} \rightarrow \mathrm{e}^{\prime}+\mathrm{K}^{+}+\mathrm{H}
$$

The cross section of the reaction is again given by Eqn. 1.10 with the proton (hyperon) 4momenta and masses replaced by those of the nucleus $\mathrm{A}$ (hypernucleus $\mathrm{H}$ ). In addition, the cross section is usually calculated by a distorted wave impulse approximation (DWIA) method. The hadron current matrix element in Eqn. 1.15 is substituted by the corresponding manyparticle matrix element between the non-relativistic nuclear and hypernuclear wave functions :

$$
T_{i f}^{\mu}=\left\langle\Psi_{\mathrm{H}}\left|\sum_{n=1}^{\mathrm{Z}} \chi_{\gamma} \chi_{\mathrm{K}^{+}}^{*} J^{\mu}(n)\right| \Psi_{\mathrm{A}}\right\rangle,
$$

where the sum runs over the $\mathrm{Z}$ target protons and $\Psi_{\mathrm{H}}\left(\Psi_{\mathrm{K}^{+}}\right)$is the many-particle translationally invariant (shell model) wave function of target nucleus (hypernucleus).

$\chi_{\gamma}$ is the virtual photon wave function, which is, the product of the wave functions of incoming and outgoing electrons in the plane wave approximation (the Coulomb distortion is neglected). $\chi_{\mathrm{K}^{+}}$is the distorted wave function of the scattered kaon. $\chi_{\mathrm{K}^{+}}$is calculated with the first order optical potential determined by [53]

1) the target nucleus density,

2) the appropriately averaged kaon-proton and kaon-neutron forward scattering amplitudes. 
All calculations are treated in the target nucleus frame. The Fermi motion of the target protons is neglected (frozen nucleon approximation), therefore the one-particle hadron current $J^{\mu}$ must be expressed in the frozen proton frame.

In the many-body system, the transition matrix of the electroproduction in Eqn. 1.16 is written as the following form [51]

$$
\begin{aligned}
M= & \epsilon_{\mu} J^{\mu}=F_{1}(\boldsymbol{\sigma} \cdot \boldsymbol{\epsilon})+i F_{2}\left(\hat{q} \times \hat{p}_{\mathrm{K}^{+}}\right) \cdot \boldsymbol{\epsilon}+F_{3}(\boldsymbol{\sigma} \cdot \hat{q})\left(\hat{p}_{\mathrm{K}^{+}} \cdot \boldsymbol{\epsilon}\right) \\
& +F_{4}\left(\boldsymbol{\sigma} \cdot \hat{p}_{\mathrm{K}^{+}}\right)\left(\hat{p}_{\mathrm{K}^{+}} \cdot \boldsymbol{\epsilon}\right)+F_{5}(\boldsymbol{\sigma} \cdot \hat{q})(\hat{q} \cdot \boldsymbol{\epsilon})+F_{6}\left(\boldsymbol{\sigma} \cdot \hat{p}_{\mathrm{K}^{+}}\right)(\hat{q} \cdot \boldsymbol{\epsilon}),
\end{aligned}
$$

The $\left(\hat{q} \times \hat{p}_{\mathrm{K}^{+}}\right) \cdot \boldsymbol{\epsilon}$ term is used instead of the term quadratic in the Pauli matrices $\left(\boldsymbol{\sigma} \cdot \hat{p}_{\mathrm{K}^{+}}\right)\{\boldsymbol{\sigma}$. $(\hat{q} \times \boldsymbol{\epsilon})\}+f_{3}(\boldsymbol{\sigma} \cdot \hat{q})\left(\hat{p}_{\mathrm{K}^{+}} \cdot \boldsymbol{\epsilon}\right)$, which is used in the amplitude Eqn. 1.16 in the CMS frame. Six amplitudes $F_{i}$ are expressed as linear combinations of covariant form factors $A_{i}[51]$. 


\subsection{Goal of the present thesis}

The present thesis aims to explore and establish the $\left(\mathrm{e}, \mathrm{e}^{\prime} \mathrm{K}^{+}\right)$reaction as a new and powerful tool for precision spectroscopic study of $\Lambda$ hypernuclei in the wide mass range. To this end, we have constructed a new kaon spectrometer and carried out a second generation $\left(\mathrm{e}, \mathrm{e}^{\prime} \mathrm{K}^{+}\right)$ spectroscopy experiment introducing a new experimental configuration for the scattered electron spectrometer ("tilt method") based on the through examination of the previous E89-009 experiment.

It is intended to demonstrate that high production rates of hypernuclear states and unprecedented hypernuclear mass resolution are realized in the experiment using the $\left(\mathrm{e}, \mathrm{e}^{\prime} \mathrm{K}^{+}\right)$reaction.

In this thesis, the ${ }^{12} \mathrm{C}$ target data that gives ${ }_{\Lambda}^{12} \mathrm{~B}$ hypernuclear spectrum is analyzed to show the performance of the experiment.

Furthermore, the binding energy of the neutron-rich hypernucleus ${ }_{\Lambda}^{7} \mathrm{He}$ was deduced and the $A=7$ iso-multiplet hypernuclear systems are discussed with a help of recent cluster-models.

The design and performance of the kaon identification detector is explained in Chapter 3. Chapter 4 describes the data analysis procedure, detector performance, optimization of the optics and evaluation of efficiencies and acceptances. Chapter 5 represents the experimental results and discusses the hypernuclear structures and cross sections of the observed hypernuclear states. The conclusion is given in Chapter 6 . 


\section{Chapter 2}

\section{Experimental apparatus}

\subsection{Overview}

The hypernuclear spectroscopic experiment via the (e,e' $\left.\mathrm{K}^{+}\right)$reaction, JLab E01-011 was carried out in Hall C, Jefferson Lab from June to October 2005.

The goal of this experiment is to determine energy levels of $s, p$-shell $\Lambda$ binding states and core excited states located between them with ${ }^{7} \mathrm{Li}$ and ${ }^{12} \mathrm{C}$ targets.

Furthermore, $\Lambda$ single particle state behavior in the $p$-shell region are observed with ${ }^{28} \mathrm{Si}$ and heavier targets, since a spacing of the LS splittings is expected to be wider as $Z$ increased, in general.

The high luminosity and high quality electron beam which is provided by the Continuous Electron Beam Facility (CEBAF) at JLab is powerful tool to observe the small cross section of the electro-magnetic interaction.

CEBAF is an electron linear accelerator, delivered $100 \%$ duty cycle continuous wave (CW) to three experimental hall; Hall A, B and C as shown in Fig. 2.1.

An electron beam of $1497 \mathrm{MHz}$ short bursts (1.67 ps) was generated at the injector, divided to $1497 \mathrm{MHz} / 3=499 \mathrm{MHz}$ bursts by RF chopper and accelerated by the south and north linacs up to maximum of $5.5 \mathrm{GeV}$.

Finally, beam was delivered to each experimental hall with a 2 ns bunch structure. After the beam was split from the accelerator beam switch yard into Hall $\mathrm{C}$, it was transported to the Hall C beamline.

During E01-011, the Hall C beamline consisted of a series of 6 beam position monitors (BPMs), 2 beam current monitors (BCMs), 8 superharps for beam position calibration and an unser monitor for BCM calibration [54], and finally reached the target as shown in Fig. 2.2.

Since splitter magnet was used to separate scattered electrons and positive kaons after the target, pass-through electrons were deflected by the 2 correction magnets into the original Hall C beam dump together with Bremsstrahlung photon.

A typical beam emittance was $2 \times 10^{-7} \mathrm{~cm} \cdot \mathrm{rad}$ with as energy spread of $\Delta E / E \leq 1.0 \times 10^{-9}$ (FWHM). This beam quality is much better than that of meson beams produced at KEK or BNL where the beam emittance is a few tens $\mathrm{cm} \cdot \mathrm{rad}$ and energy spread around a few $\%$.

In this chapter, the experemental settings are presented in Section 2.2, the experimental equipment, the expected singles rate and energy resolution of mass spectra, trigger logic and data aquisition system in Section 2.3, 2.4, 2.5 and 2.6, respectively.

Finally, this chapter is concluded with the data summary in Section 2.7. 


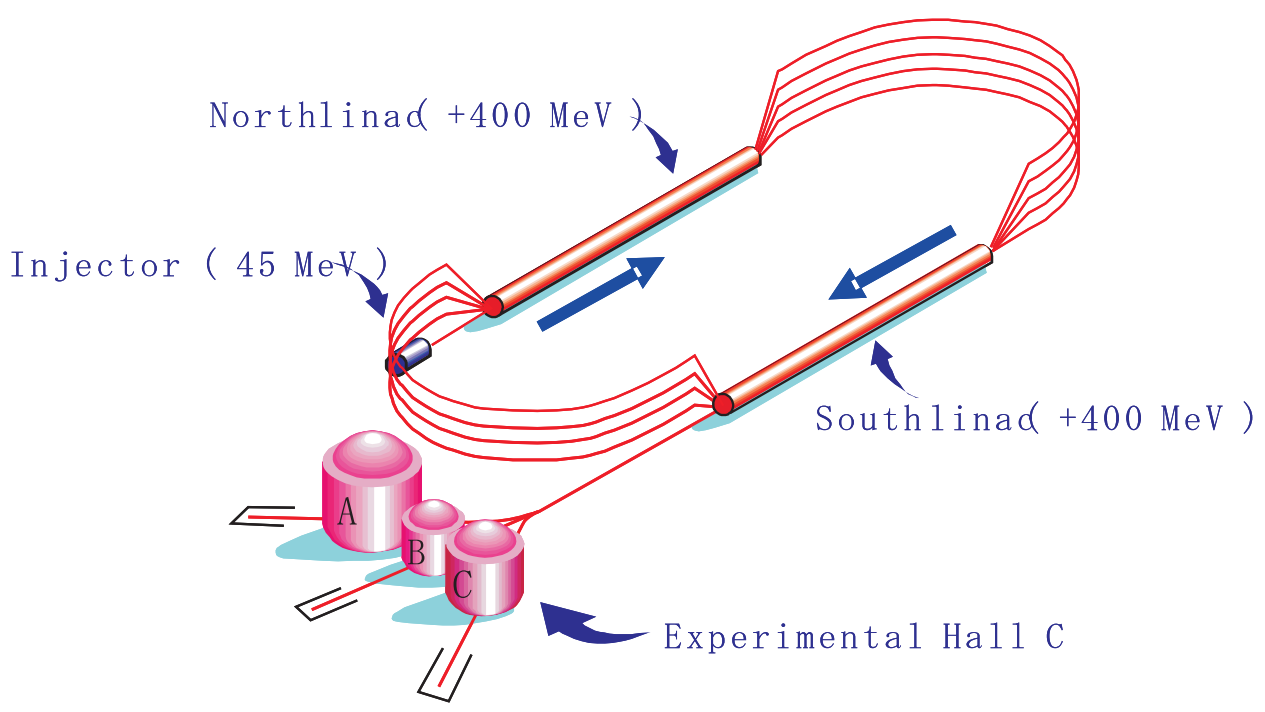

Figure 2.1: Schematic of CEBAF and experimental halls at JLab.

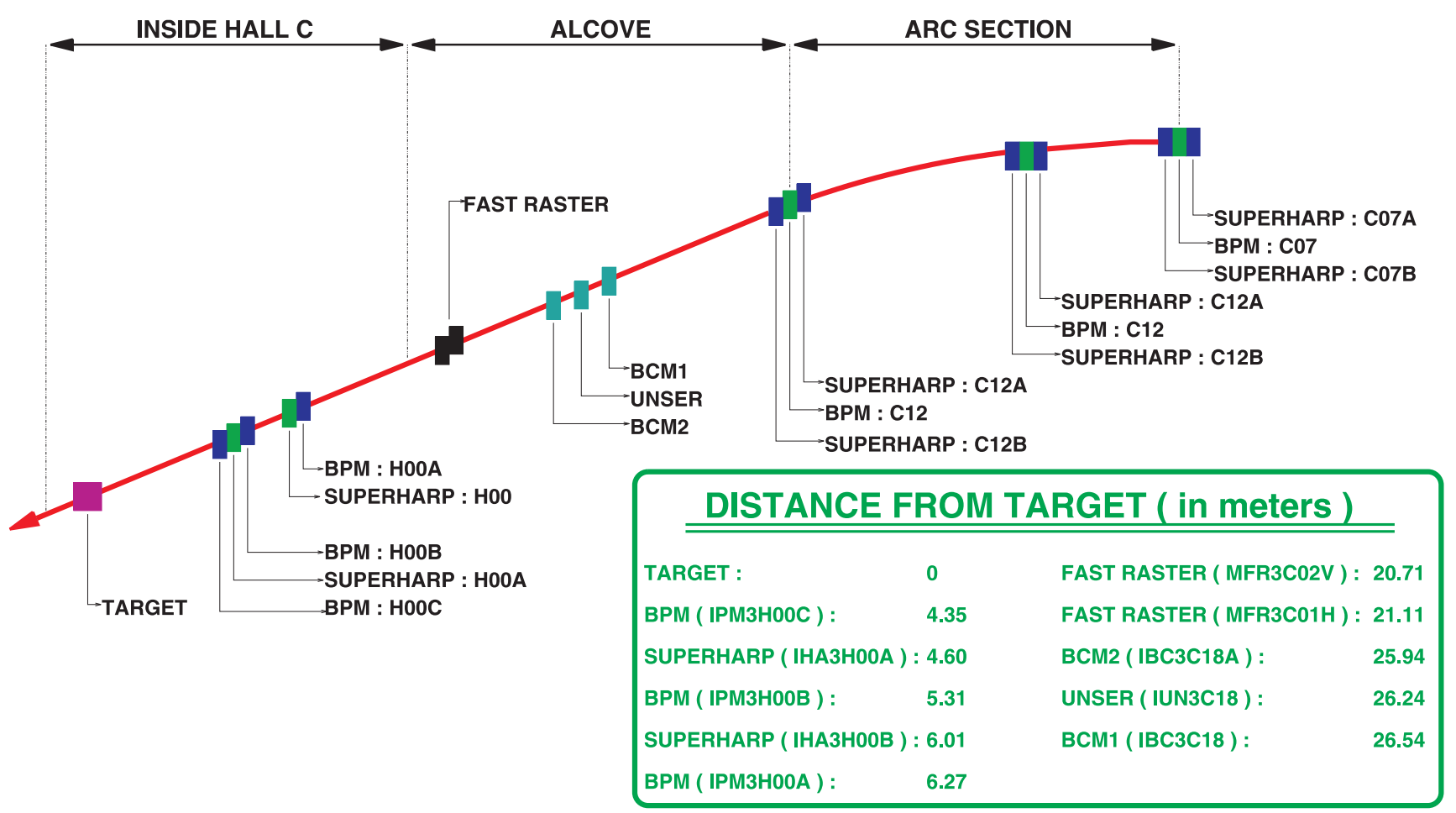

Figure 2.2: Hall C beamline. 


\subsection{Experimental condition}

\subsubsection{Kinematics}

A schematic of the $\left(\mathrm{e}, \mathrm{e}^{\prime} \mathrm{K}^{+}\right)$reaction is shown in Fig. 2.3.

First, a primary electron is applied to a target nucleus with beam energy of $1.8 \mathrm{GeV}$.

Then a virtual photon with energy of $1.5 \mathrm{GeV}$ hits a proton and emits a kaon with $1.2 \mathrm{GeV} / c$ , while a scattered electron goes out of the target nucleus with momentum of $0.3 \mathrm{GeV} / c$.

The total cross section of $\Lambda$ photoproduction as a function of the incident real photon energy was obtained by the SAPHIR group as shown in Fig. 2.4 [55]. Therefore, the virtual photon energy is set as $\sim 1.5 \mathrm{GeV}$, since total cross section of the kaon photoproduction is expected to be maximum around $1.1 \sim 1.5 \mathrm{GeV}$ as shown in Fig. 2.4.

Next, we designed the kaon spectrometer (HKS) to cover a central kaon momentum of 1.2 $\mathrm{GeV} / c$, and to detect kaons with scattering angles of $1^{\circ} \sim 13^{\circ}$.

The cross section of the ${ }_{\Lambda}^{12} \mathrm{~B}$ ground state doublet as a function of the kaon scattering angle with our experimental settings is obtained by a DWIA calculation [49] as shown in Fig. 2.5. It is found that the maximum cross section is given with kaon forward angle detection, and gradually decreased with increasing angle.

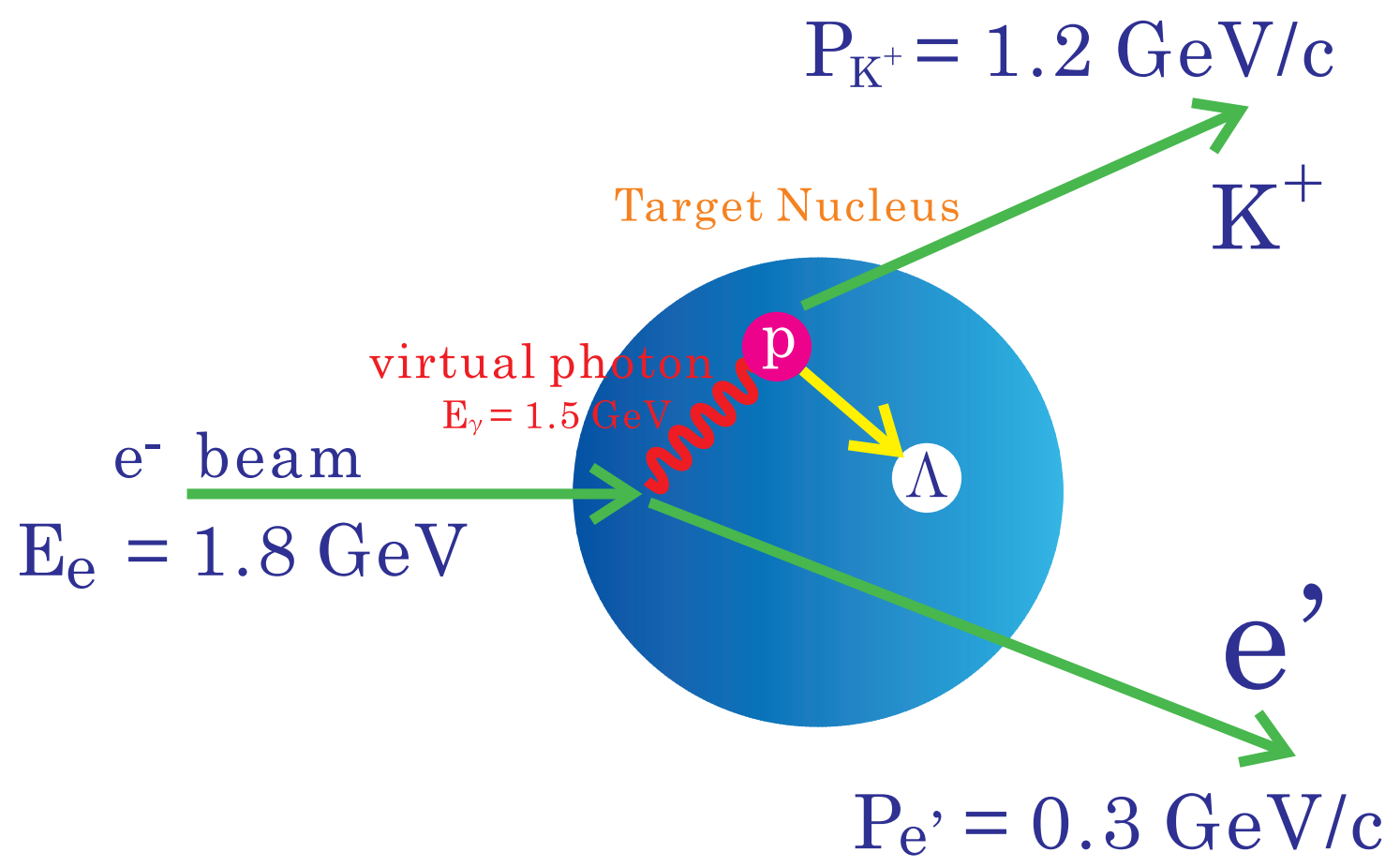

Figure 2.3: Schematic drawing of the $\left(\mathrm{e}, \mathrm{e}^{\prime} \mathrm{K}^{+}\right)$reaction.

On the other hand, we needed to use an existing electron spectrometer (Enge) with $0.3 \mathrm{GeV} / c$ central momentum. Finally, the incident electron beam energy was chosen to be $1.8 \mathrm{GeV}$. The detection angle of the scattered electron was set as $3.7^{\circ} \sim 5.7^{\circ}$.

The virtual photon flux in Eqn. 1.12 is rewitten with the incoming elecron energy $E_{\mathrm{e}}$, the electron scattering angle $\theta_{\mathrm{e}}$ and the virtual photon energy $\omega$ (the same variable definition as 


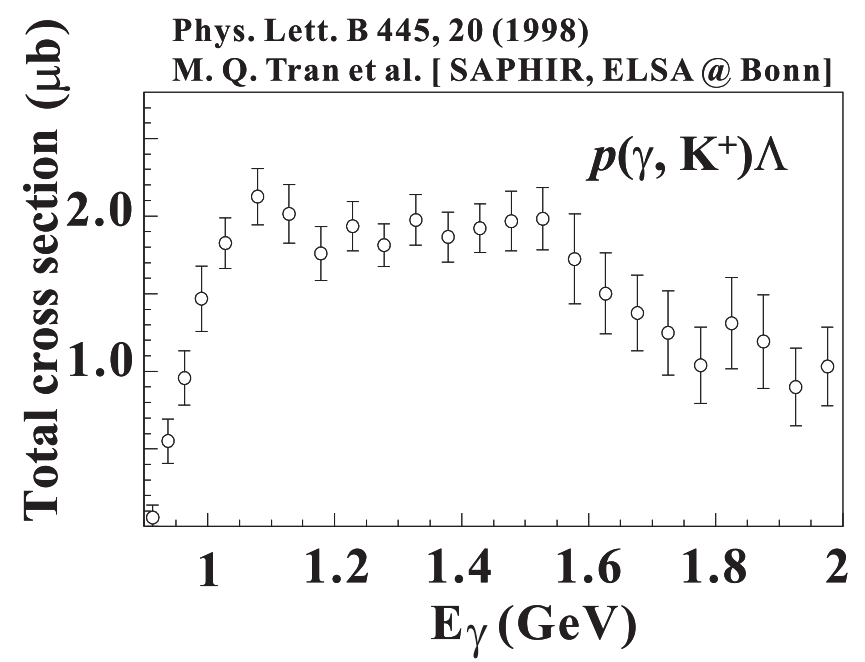

Figure 2.4: Total cross section of the $\mathrm{p}\left(\gamma, \mathrm{K}^{+}\right) \Lambda$ reaction.

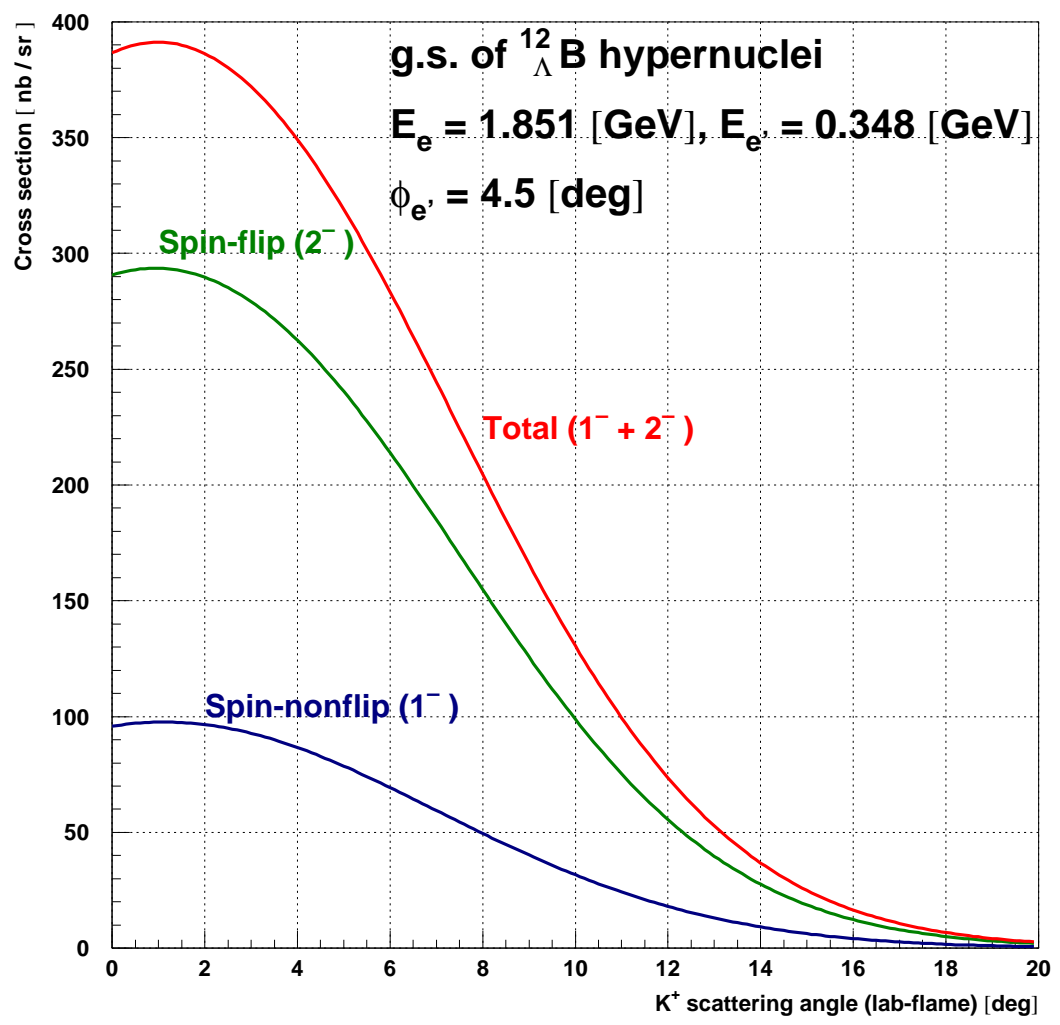

Figure 2.5: Scattered kaon angular distribution of the photo production of ${ }_{\Lambda}^{12} \mathrm{~B}$ ground state doublet from a DWIA calculation [49] assuming $E_{\text {beam }}=1.851[\mathrm{GeV}], E_{\mathrm{e}}{ }^{\prime}=0.348[\mathrm{GeV}]$ and $\phi_{\mathrm{e}^{\prime}}=4.5[\mathrm{deg}]$. 
discussed in Chapter 1.5) as [56]:

$$
\begin{aligned}
\Gamma\left(E_{\mathrm{e}}, \omega, \theta_{\mathrm{e}}\right)= & \frac{\alpha}{4 \pi^{2} \omega}\left[\frac{E_{\mathrm{e}}^{2}+E_{\mathrm{e}}^{2}}{2 E_{\mathrm{e}}^{2}}\left(\frac{m_{\mathrm{e}}^{2} \omega^{2}}{4 E_{\mathrm{e}}^{2} E_{\mathrm{e}}^{2}}+\sin ^{2} \frac{\theta_{\mathrm{e}}}{2}\right)^{-1}-\frac{E_{\mathrm{e}}}{E_{\mathrm{e}}} \frac{m_{\mathrm{e}}^{2} \omega^{2}}{4 E_{\mathrm{e}}^{2} E_{\mathrm{e}}^{2}}\left(\frac{m_{\mathrm{e}}^{2} \omega^{2}}{4 E_{\mathrm{e}}^{2} E_{\mathrm{e}}^{2}}+\sin ^{2} \frac{\theta_{\mathrm{e}}}{2}\right)^{-2}\right. \\
& \left.-\frac{\left(E_{\mathrm{e}}+E_{\mathrm{e}}\right)^{2}}{4 E_{\mathrm{e}}^{2}}\left(\frac{\omega^{2}}{4 E_{\mathrm{e}} E_{\mathrm{e}}}+\sin ^{2} \frac{\theta_{\mathrm{e}}}{2}\right)^{-1}\right]
\end{aligned}
$$

Thus, the virtual photon flux $\Gamma$ reachs maximum at,

$$
\theta_{\mathrm{e}}=\sin ^{-1}\left(\frac{m_{\mathrm{e}} \omega}{4 E_{\mathrm{e}} E_{\mathrm{e}}}\right)
$$

The angular distribution of virtual photon flux assuming our experimental condition with ${ }^{12} \mathrm{C}$ target is described in Fig. 2.6.

The angular distribution of Bremsstrahlung photons is overlaid in Fig. 2.6.

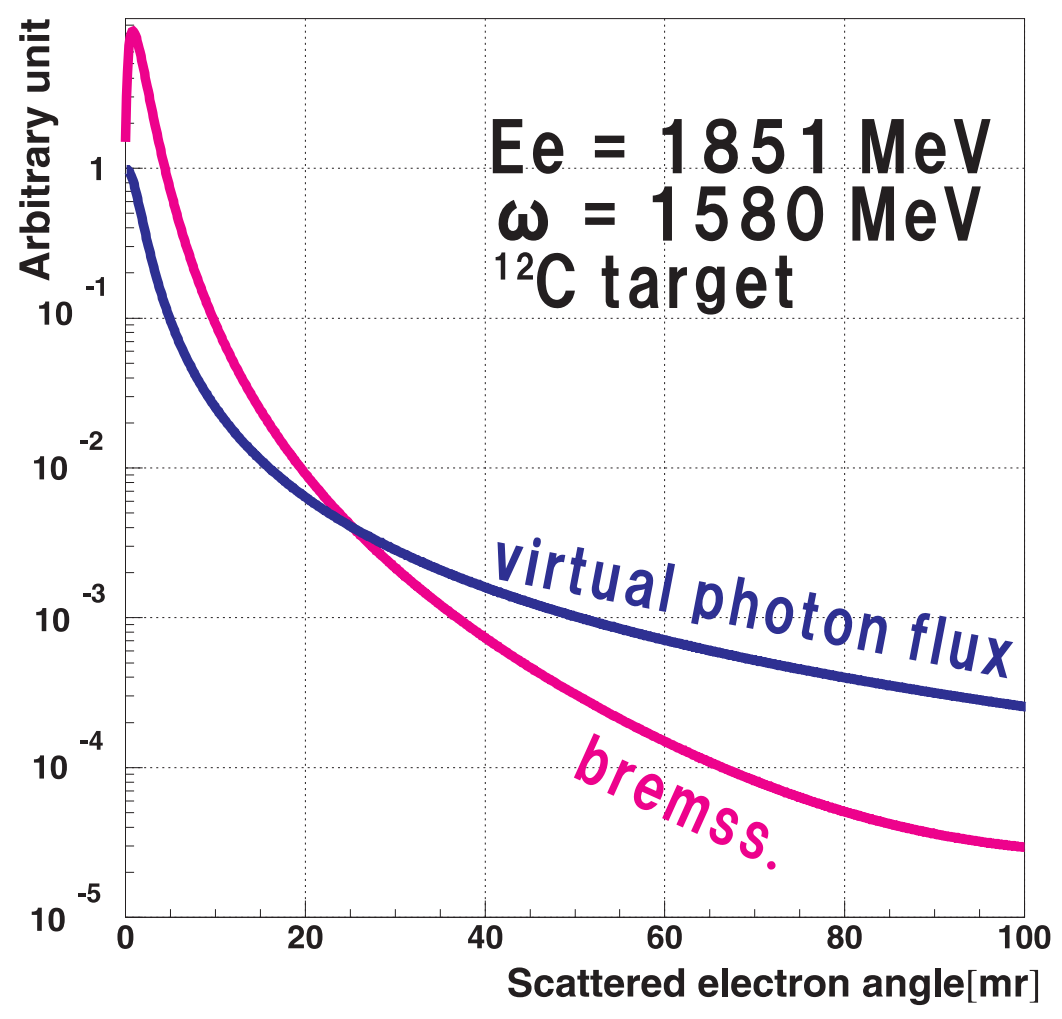

Figure 2.6: Angular distribution of the virtual photon flux assuming $E_{\mathrm{e}}=1.851 \mathrm{GeV}, \omega=1.580$ $\mathrm{GeV}$.

Therefore, both scattered electrons and kaons need to be detected as forward angles as much as possible in order to cover the $\Lambda$ hypernuclear production cross section maximum.

However, both Bremsstrahlung electrons and positron associated with pair creation distribute extremly forward, thus optimization of the detection angle is required as discussed in 2.2.2.

Calculated momentum correlations of scattered electron and kaon are shown in Fig. 2.7 for $\Sigma^{0}, \Lambda,{ }_{\Lambda}^{12} \mathrm{~B}$ ground states and the ${ }_{\Lambda}^{28} \mathrm{Al}$ ground state region with a beam energy of $1.851 \mathrm{GeV}$. Here, the abinding energies (as the basis for "core nucleus mass $+\Lambda$ mass") for ${ }_{\Lambda}^{12} \mathrm{~B}$ and ${ }_{\Lambda}^{28} \mathrm{Al}$ 


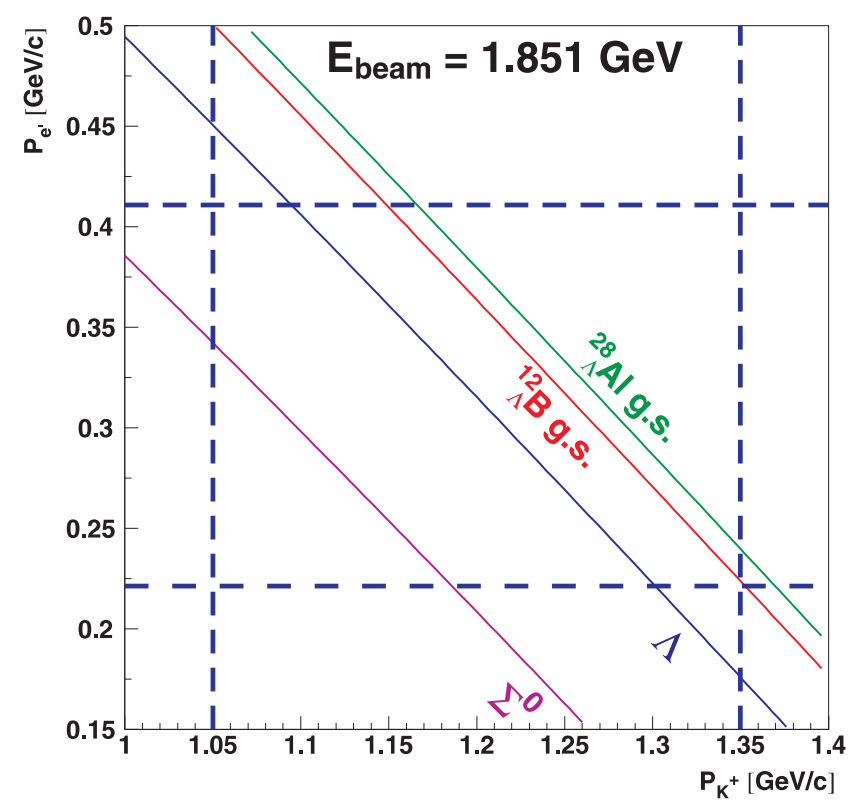

Figure 2.7: Momentum acceptance of the kaon and scattered electron spectrometers. Beam energy is set as $1.851 \mathrm{GeV}$, and binding energy of ${ }_{\Lambda}^{12} \mathrm{~B}$ and ${ }_{\Lambda}^{28} \mathrm{Al}$ ground states are assumed as $-11.37 \mathrm{MeV}$ [57] and $-16.92 \mathrm{MeV}$ [58], respectively.

ground states are assumed as -11.37 [57], -16.92 [58] MeV, respectively.

The designed central momentum and acceptance of the Enge and HKS are $316.0 \mathrm{MeV} / c \pm$ $30.0 \%, 1200.0 \mathrm{MeV} / c \pm 12.5 \%$, respectively, as marked by dashed lines in Fig. 2.7.

The acceptance of both the two spectrometers fully cover not only the ${ }_{\Lambda}^{12} \mathrm{~B}$ and ${ }_{\Lambda}^{28} \mathrm{Al}$ bound regions, but also cover the $\Lambda$ and $\Sigma^{0}$ kinematics regions. 


\subsubsection{Tilt method principal}

The scattering angles of Bremsstrahlung electrons are further forward than those associated with virtual photon production (see Fig. 2.6). Fig. 2.8 shows schematic side view of the Enge and the splitter magnet configuration for the E89-009 (left) and the E01-011 (right).
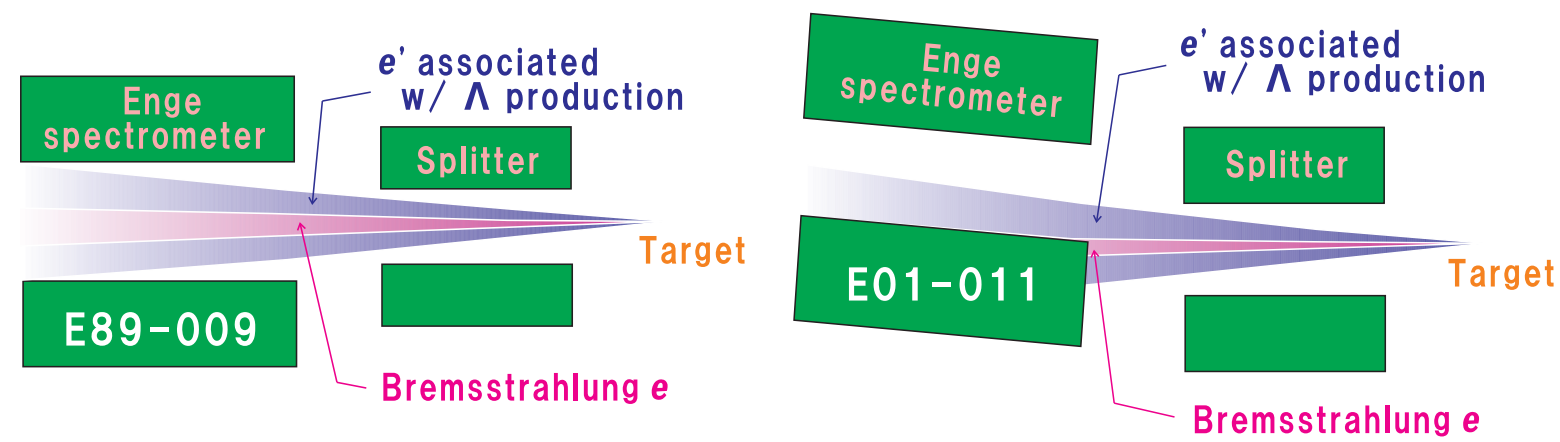

Figure 2.8: Schematic comparison of the splitter and Enge configuration of E89-009 and E01-011.

As shown in Fig. 2.8 (left) the Enge was placed in the same plane with the splitter's dispersion, the trigger of the scattered electron arm was dominated with high rate Bremsstrahlung electrons.

Therefore, the Data Acquisition (DAQ) was at its limit, the counting rate of the $\Lambda$ particle was suppressed and the signal/accidental ratio was decreased.

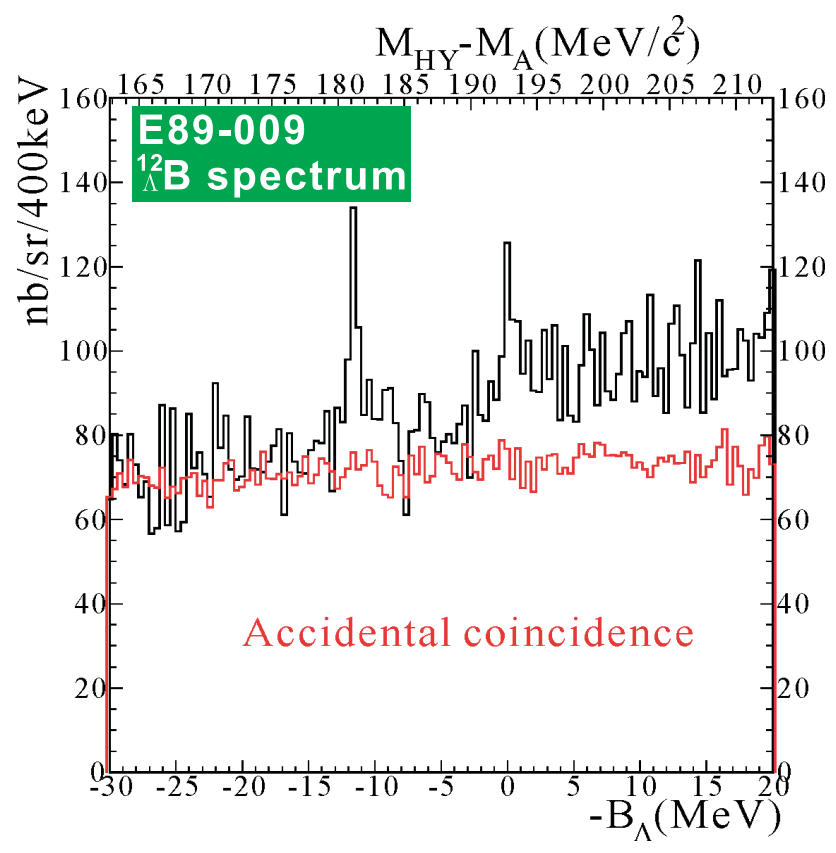

Figure 2.9: ${ }_{\Lambda}^{12} \mathrm{~B}$ spectrum measured in the JLab E89-009 experiment for net 90 days data taking.

Based on the E89-009 experience, the Enge is tilted vertically by $7.5^{\circ}$ and shifted above the splitter's dispersion plane as shown in Fig. 2.8 (right), suppressing the Bremsstrahlung electrons at the hardware level. 


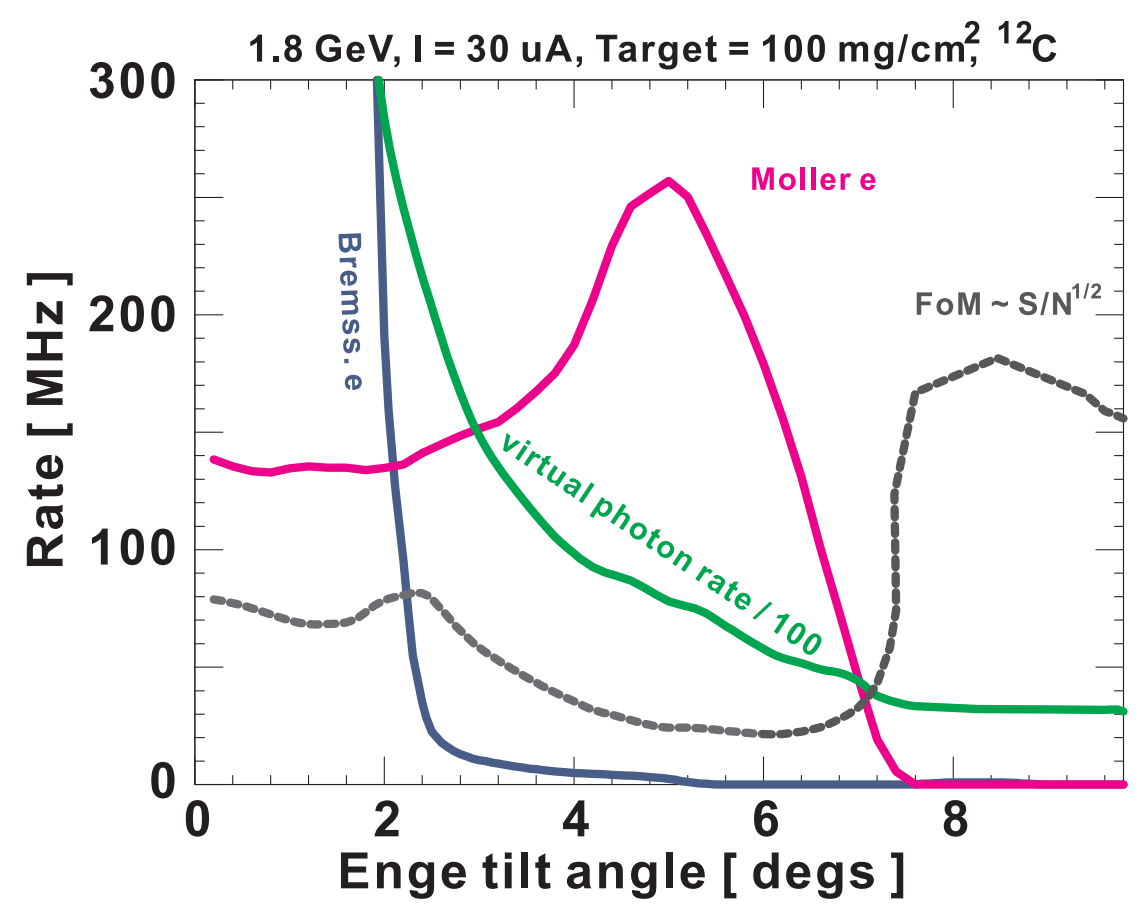

Figure 2.10: Enge tilt angle dependence of the expected rates. A beam current of $30 \mu \mathrm{A}$ and 100 $\mathrm{mg} / \mathrm{cm}^{2}$ of carbon target are assumed. The figure of merit (FoM) is defined as $S / N^{1 / 2}$, where $S$ is the virtual photon flux and $N$ the sum of Bremsstrahlung electrons and Møller scattering electrons. For convenience of graphical view, the virtual photon flux is multiplied by 0.01 and FoM by arbitrary factor.

The expected counting rate of the three physics processes i.e. Bremsstrahlung (Bremss), Møller (Mo) scattered electrons, electrons associated with the virtual photon production (VP) and a Figure of Merit (FoM) are scanned with scattering angle at a target point as shown in Fig. 2.10, where FoM was defined as:

$$
\begin{aligned}
\text { FoM } & =\frac{S}{\sqrt{N}} \\
S & =(\text { rate of } \mathrm{VP}) \\
N & =(\text { rate of Bremss })+(\text { rate of } \mathrm{Mo})
\end{aligned}
$$

In Fig. 2.10, a $100 \mathrm{mg} / \mathrm{cm}^{2}$ thick ${ }^{12} \mathrm{C}$ target with a $30 \mu \mathrm{A}$ beam current is assumed.

According to the FoM calculation, it is found that the contribution from background electrons to the $\mathrm{S} / \mathrm{N}$ ratio becomes small around a tilt angle of $7^{\circ} \sim 8^{\circ}$.

Precise tilt angle and shift length combination is determined by RAYTRACE calculation as shown in Fig. 2.11.

It shows an expected detection rate map of the virtual photon associated electron (upper), Møller (middle) and Bremsstrahlung (lower) scattered electron. The $\mathrm{x}$ and $\mathrm{y}$ axes represent the Enge tilt angle and shift length from the splitter dispersion plane, respectively.

This tilt method has the possibility to suppress some fraction of the electrons which should be detected. However, this issue can be avoided by increasing the beam current and target thickness. 


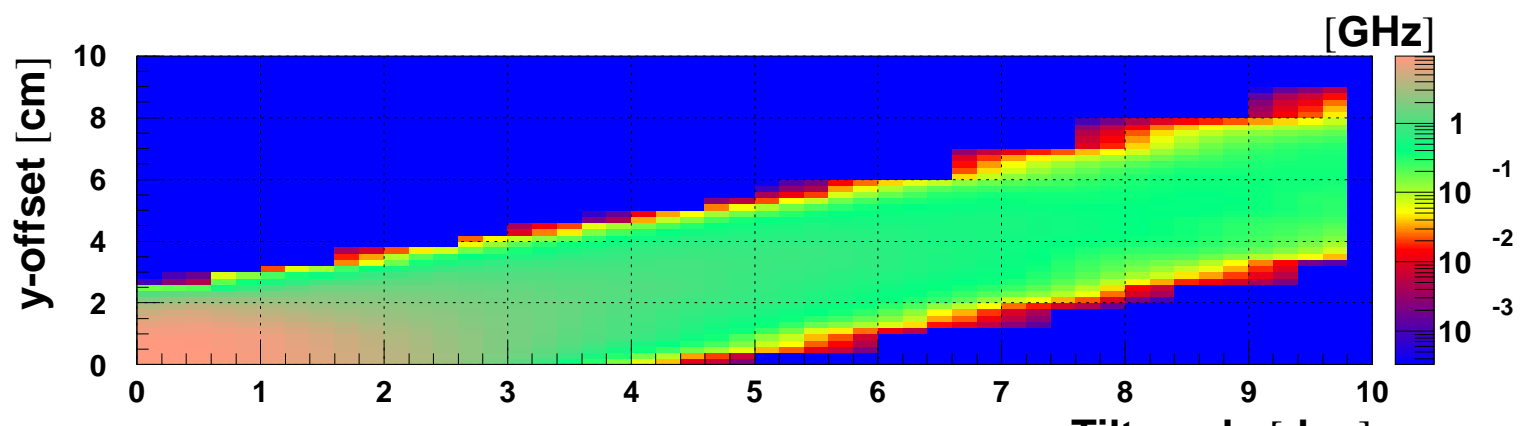

Virtual photon flux

Tilt angle [deg]
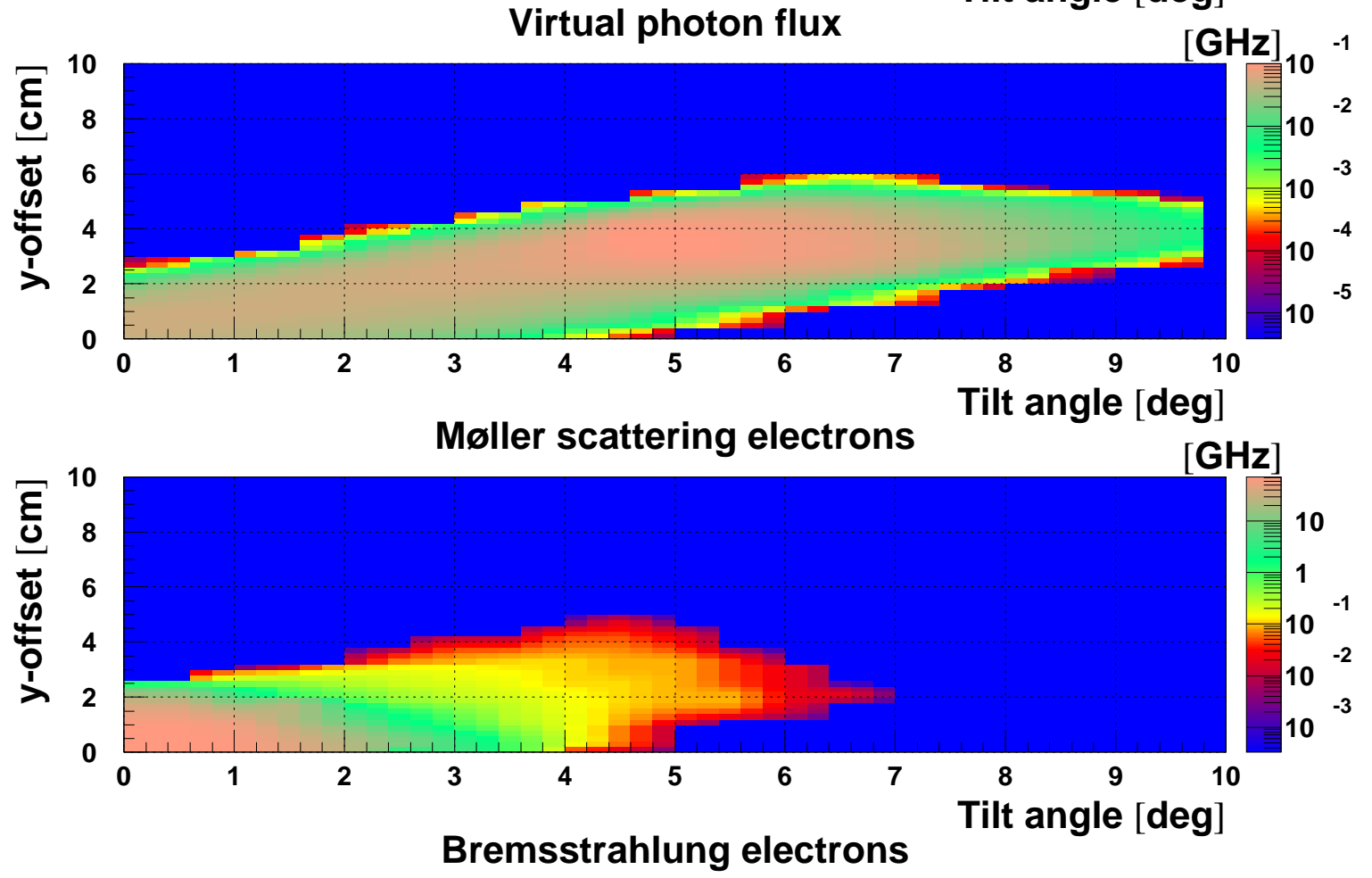

Figure 2.11: A relative electron rate dependence on the Enge tilt angle and vertical offset for Bremsstrahlung, Møller scattering and the electrons associated with hypernuclear production for 100 $\mathrm{mg} / \mathrm{cm}^{2}$ of ${ }^{12} \mathrm{C}$ target with $30 \mu \mathrm{A}$ electron beam. The absorption cross section for the virtual photon is assumed to be $100[\mathrm{nb} / \mathrm{sr}]$. 


\subsection{The HKS-Enge spectrometer system}

The E01-011 hypernuclear spectroscopy system is established to achieve high mass energy resolution for $\Lambda$ hypernuclear spectroscopy. It consists of three magnets, a splitter magnet, the Enge split pole magnet (Enge) and the High resolution Kaon Spectrometer as shown in Fig. 2.12 .

The incident electron $(\sim 1.8 \mathrm{GeV})$ interacts with the target nucleus to create a hypernucleus,
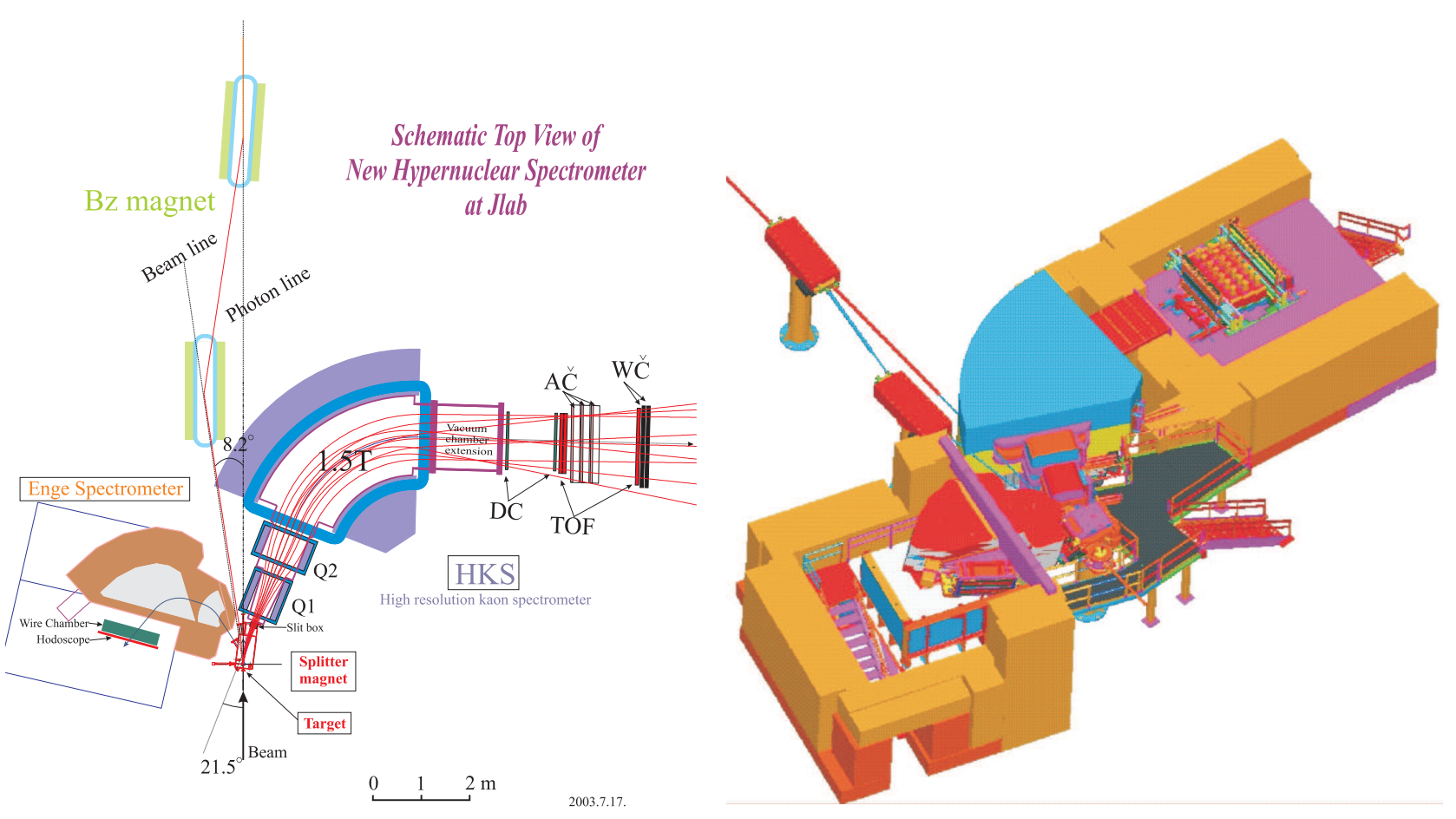

Figure 2.12: Top (left) and bird's eye (right) view of the JLab E01-011 experimental setup.

a scattered electron and a positive kaon.

Scattered particles are separated according to their electronic charge just after the target by the splitter magnet in order to detect them at a forward angle ; a requirement explained in last section.

After that, the $\sim 300 \mathrm{MeV} / c$ scattered electrons are detected by the Enge, and the $\sim 1.2 \mathrm{GeV} / c$ kaon are detected by the HKS which consists of two quadrupole magnets and a dipole.

Table 2.1 summarizes the configuration of the E01-011 hypernuclear spectroscopy system. A detail description of each spectrometer's components is given in the following sections.

\subsubsection{Splitter magnet and target chamber}

The splitter magnet (Fig. 2.13) is used to deflect charged particles into the Enge/HKS spectrometers.

This spectrometer is a C-type, normal conducting dipole magnet with $15.24 \mathrm{~cm}$ gap width.

A target chamber with a target ladder is installed into the gap.

The splitter magnet is operated by remote control, and its field is monitored by a hall probe (Group3 DTM-151 [59]). 
Table 2.1: E01-011 experimental condition and spectrometer specification.

\begin{tabular}{lc}
\hline \hline Beam condition & $1.851 \mathrm{GeV}$ \\
Beam energy & $<1 \times 10^{-4}$ \\
Beam momentum stability & \\
General configuration & Splitter $+\mathrm{HKS}+$ Enge \\
HKS & \\
Configuration & QQD and horizontal bend \\
Central momentum & $1.2 \mathrm{GeV} / c$ \\
Momentum acceptance & $\pm 12.5 \%$ \\
Momentum resolution $(\Delta p / p)$ & $2 \times 10^{-4}(\mathrm{FWHM})$ \\
& $($ beam spot size $0.05 \mathrm{~mm}$ assumed $)$ \\
Solid angle & $20 \mathrm{msr}$ with a splitter \\
Kaon detection angle & Horizontal $: 7^{\circ}\left(1^{\circ} \sim 13^{\circ}\right)$ \\
& \\
Enge & \\
Central momentum & $0.276 \mathrm{GeV} / c$ \\
Momentum acceptance & $\pm 30 \%$ \\
Momentum resolution $(\Delta p / p)$ & $4 \times 10^{-4}(\mathrm{FWHM})$ \\
Solid angle & 7 msr with a splitter \\
Tilt angle & Vertical : $7.75^{\circ}$ \\
Angular acceptance & $3.7^{\circ} \sim 5.7^{\circ}$ \\
\hline \hline
\end{tabular}

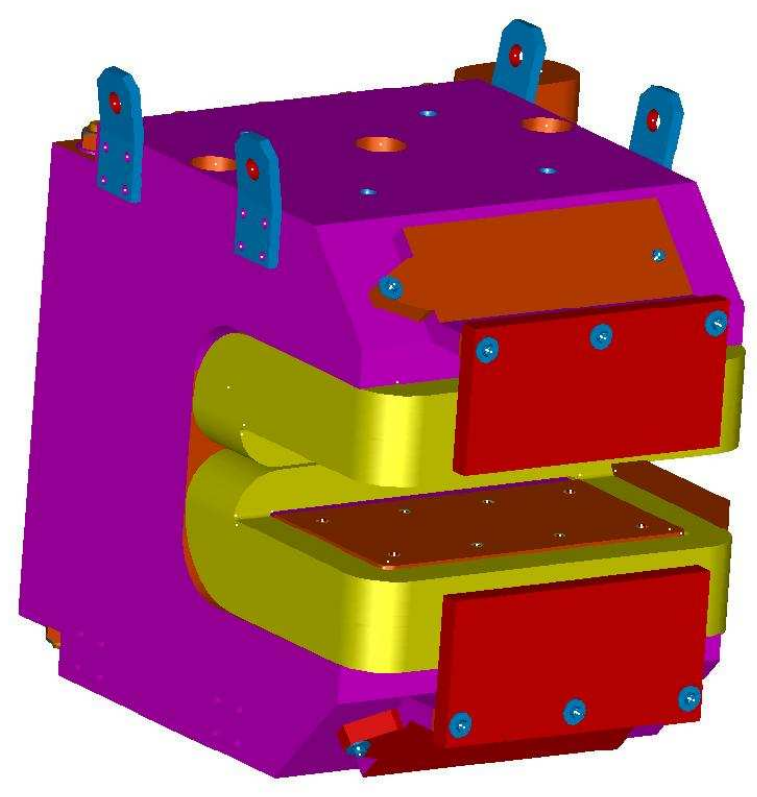

Figure 2.13: Schematic design of the splitter magnet. 

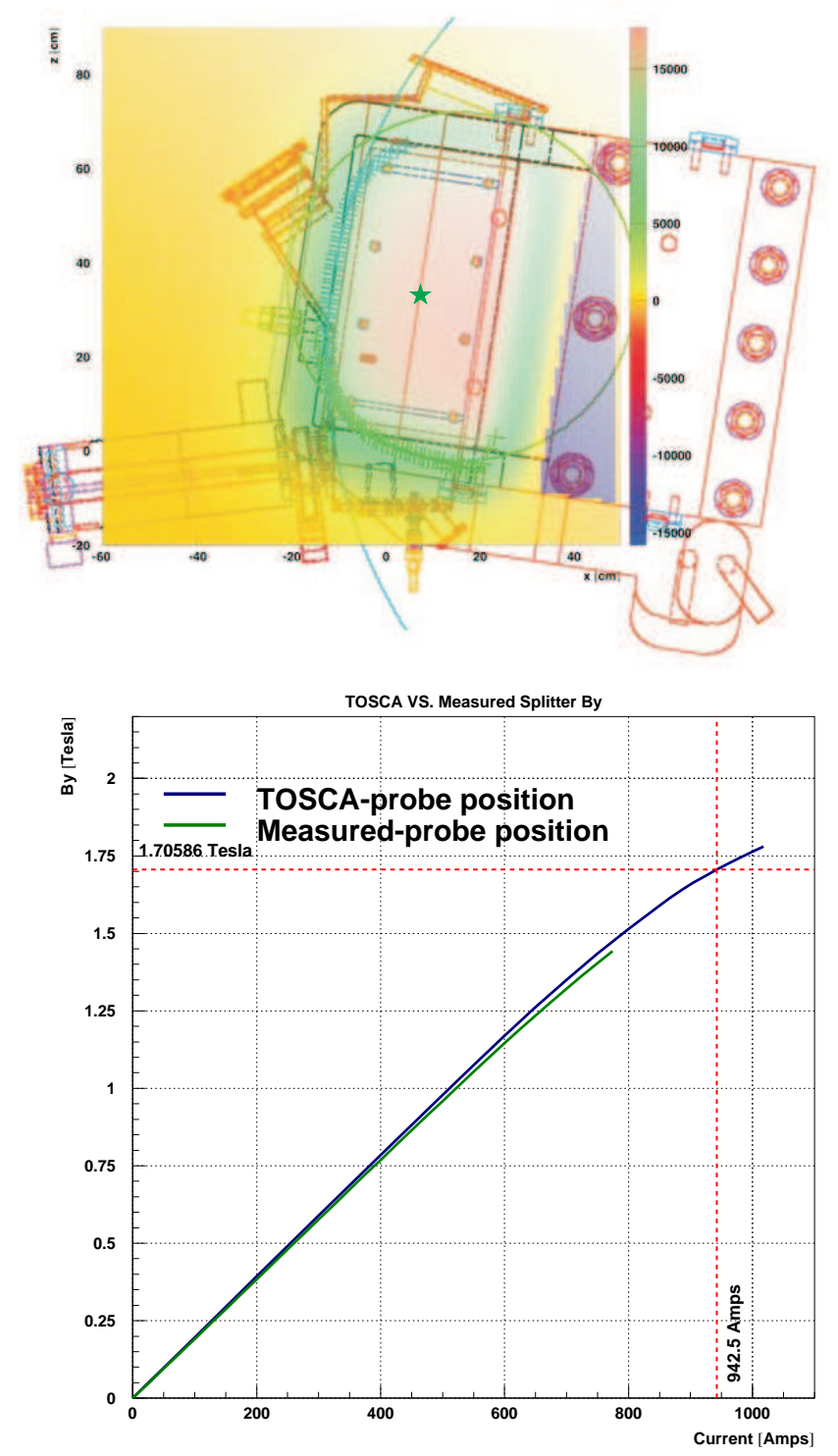

Figure 2.14: Schematic drawing of the splitter magnet (left). Magnetic field on the mid-plane of the splitter magnet (marked with star in the left figure) is measured and compared with a TOSCA calculation (right). 
The magnetic field on the mid-plane of the splitter magnet was measured with a hall probe before the beginning of the experiment at currents of up to $780 \mathrm{~A}$. However, the actual central field was exceeded beyond the measured range because of last minute changes made when tuning the beam. Therefore, the central field of the splitter magnet was deduced by a calculated value with TOSCA. The measured and calculated central fields of the splitter magnet are compared in Fig. 2.14 (right).

The target ladder is made of aluminum and able to hold maximally 6 targets with an area of $1.5 \times 1.5 \mathrm{~cm}^{2}$ each.

The ladder is moved by remote control and monitored by a camera. The targets mounted on the ladder during E01-011 experiment are listed up on Table 2.3.

Table 2.2: Splitter magnet parameters

\begin{tabular}{lc}
\hline \hline Item & Parameter \\
\hline Pole gap width & $15.24 \mathrm{~cm}$ \\
Max. field & $1.8 \mathrm{~T}$ \\
Weight & $6.35 \mathrm{ton}$ \\
\hline \hline
\end{tabular}

Table 2.3: The list of targets used in E01-011.

\begin{tabular}{ccc}
\hline \hline Target & Thickness $\left[\mathrm{mg} / \mathrm{cm}^{2}\right]$ & Note \\
\hline $\mathrm{CH}_{2}$ & 465 & Purity: $92.4 \%$ \\
Nat. ${ }^{7} \mathrm{Li}$ & 189 & Purity: $99 \%$ \\
${ }^{9} \mathrm{Be}$ & 188 & Isotopically enriched to $\sim 99.9 \%$ \\
${ }^{10} \mathrm{~B}$ & 114 & Purity: $98.9 \%$ \\
Nat. ${ }^{12} \mathrm{C}$ & 100 & Enriched to $\sim 99.9 \%$ \\
${ }^{28} \mathrm{Si}$ & 65 & Enriched to $\sim 99.7 \%$ \\
${ }^{\text {Nat. }}{ }^{51} \mathrm{~V}$ & 59.6 & Purity : $99.9 \%$ \\
${ }^{89} \mathrm{Y}$ & 56.0 & \\
\hline \hline
\end{tabular}




\subsubsection{Enge split pole spectrometer (Enge)}

Enge is a split pole, normal conducting dipole magnet with a focal plane well defined on a dispersive plane.

The spectrometer was designed in the 1960's to obtain two dimensional focusing over the full momentum range of $p_{\max } / p_{\min } \approx 2.8$ (energy range $E_{\max } / E_{\min } \approx 8$ ) [60].

Originally, the focal plane aberration of the second order was zero or very small over the entire energy range if the spectrometer was placed on the plane which is the same with splitter magnet's dispersion plane.

However, because of the introduction of the tilt method for the spectrometer, higher order terms including angular components need to be involved in the focal plane optics calculation. The program RAYTRACE [61] was used to calculate the optics and a momentum resolution of $\Delta p=\left(p-p_{c}\right) / p_{c} \sim 10^{-4}$ was confirmed for tilt method configuration before the experiment.

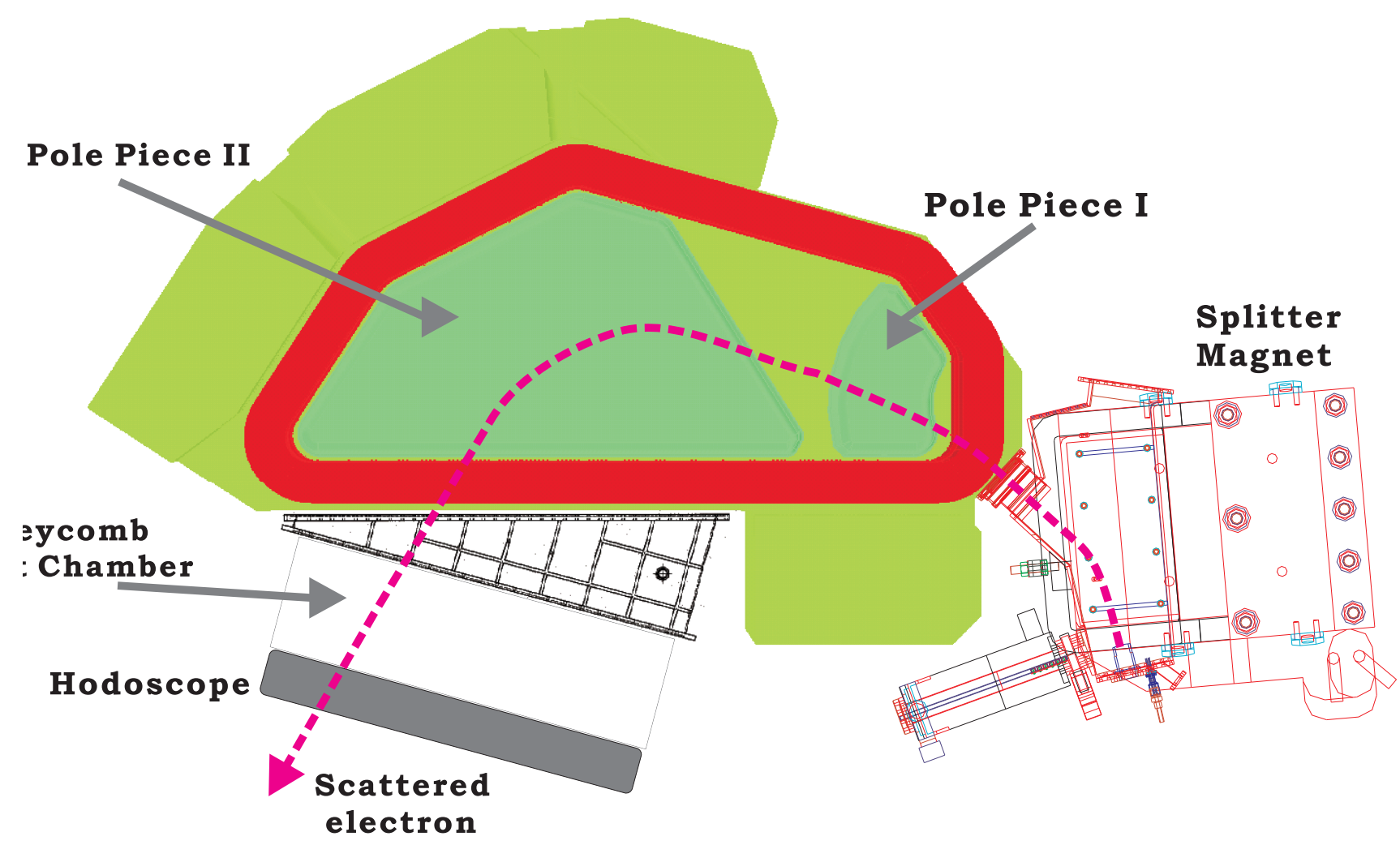

Figure 2.15: Schematic of the Enge.

Table 2.4 shows the main characteristics of the magnet. Fig. 2.16 shows Enge BI curve which was measured before the previous experiment E89-009 in the Test Lab at JLab [62].

\section{Enge drift chamber}

The Enge drift chamber (EDC) is mounted directly on the exit of Enge.

Since we applied the tilt method for the Enge, the original symmetry of the optics on the dispersion plane has already been destroyed. Thus it is most important key to measure the incident angle as well as the position on the focal plane.

The EDC is designed as a honeycomb cell structure to meet the requirements of large incident angle, and a momentum resolution of $4 \times 10^{-4}(\mathrm{FWHM})$. 
Table 2.4: Enge magnet parameters

\begin{tabular}{lc}
\hline \hline Item & Parameter \\
\hline Pole gap & $46.6 \mathrm{~mm}$ \\
Mean radius & Max. $1100 \mathrm{~mm}$ \\
Max. field & $1.8 \mathrm{~T}$ \\
Number of coils & 2 \\
Turns per coil & 100 \\
Water circuits per coil & 5 \\
Max. current & $500 \mathrm{~A}$ \\
Max. power & $65 \mathrm{~kW}$ \\
Cooling water & $50 \mathrm{l} / \mathrm{min}$. \\
Water pressure & $150 \mathrm{PSI}$ \\
Weight & $54.43 \mathrm{ton}$ \\
\hline
\end{tabular}

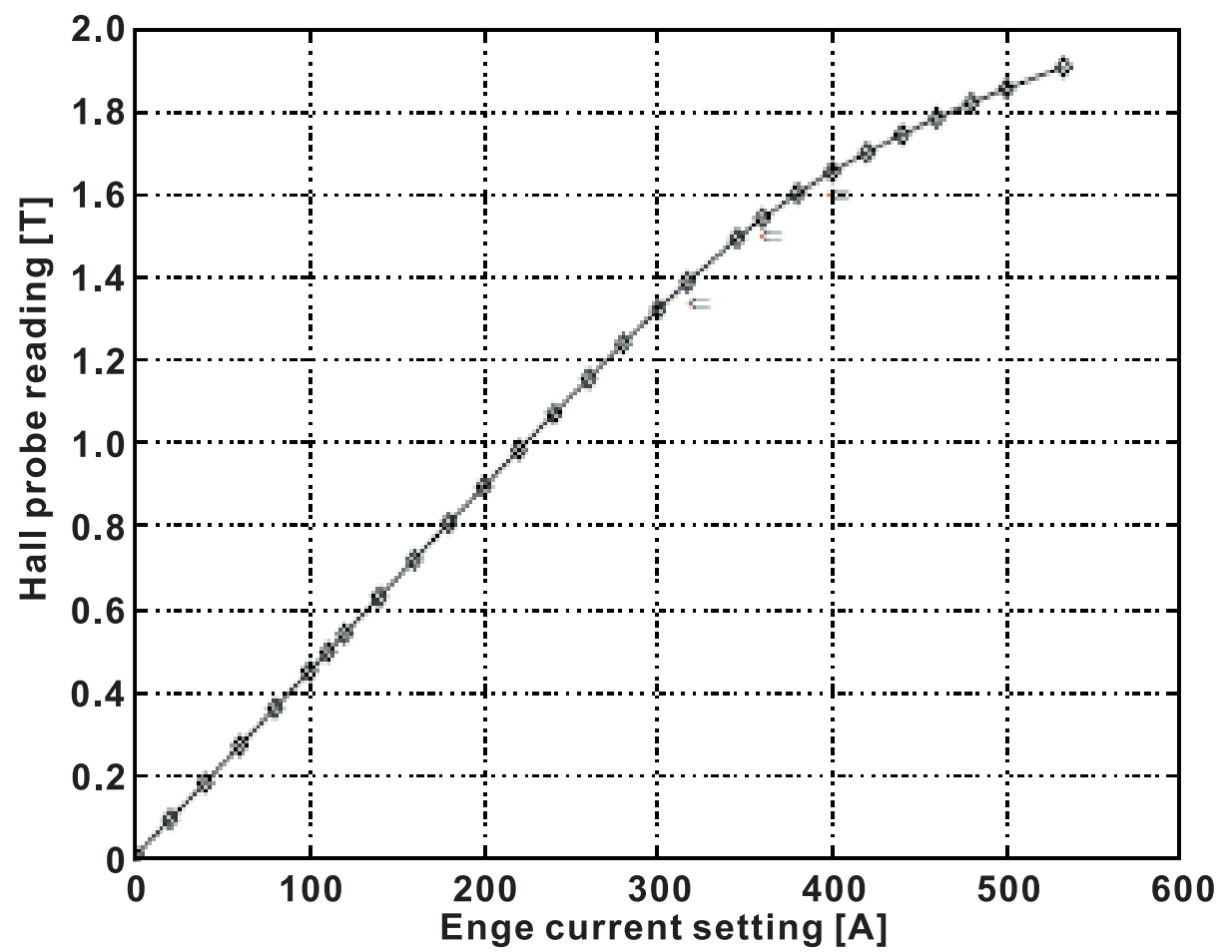

Figure 2.16: Enge BI excitation curve measured in the Test Lab at JLab [62]. 


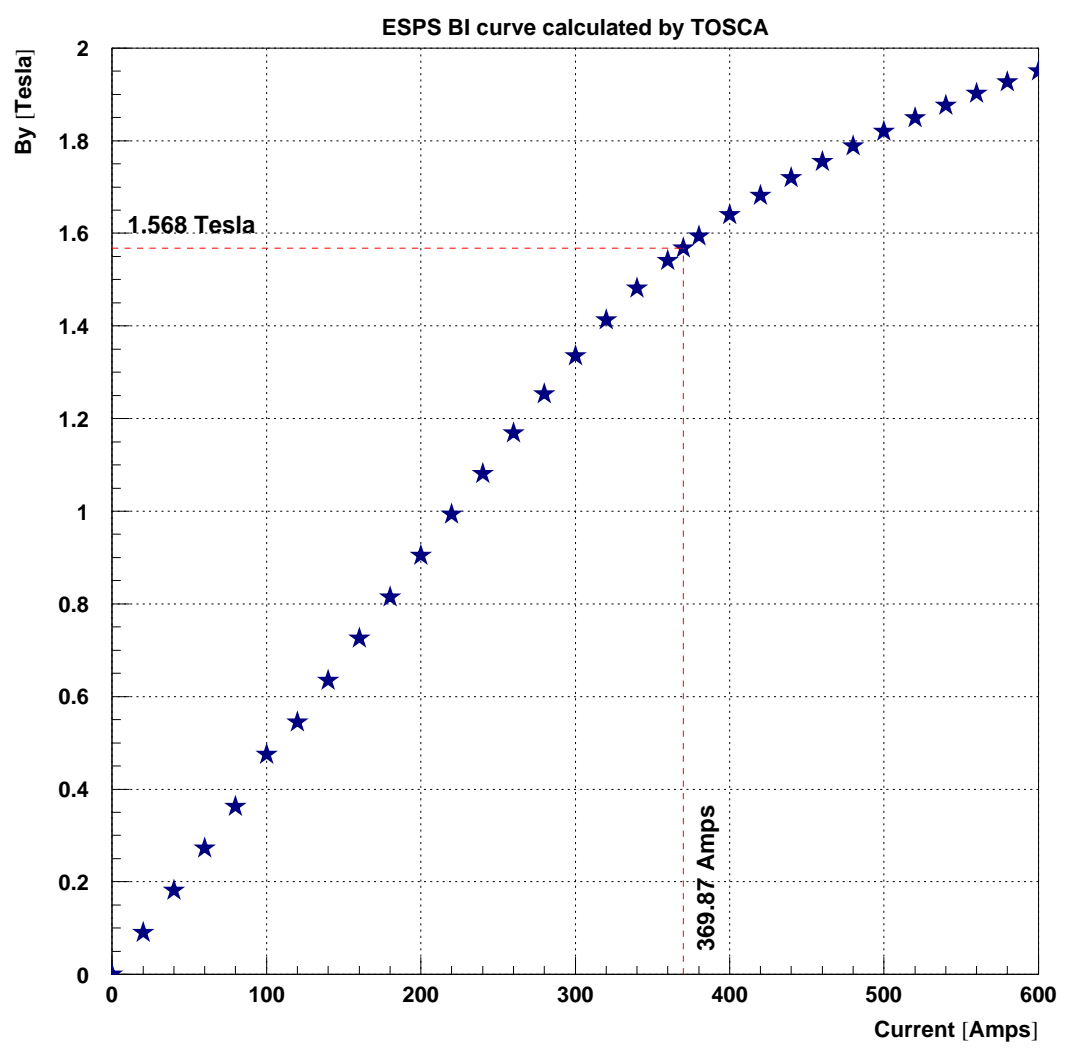

Figure 2.17: Enge BI excitation curve calculated by TOSCA.

The EDC consists of 10 layers of sense wires ( $\phi 20 \mu \mathrm{m}$ gold-plated tungsten $)$ and their hexagonal field wires ( $\phi 80 \mu \mathrm{m}$ gold-plated tungsten); xx', uu', xx' vv' and xx', and 4 layers of shield wires ( $\phi 80 \mu \mathrm{m}$ gold-plated tungsten).

The $\mathrm{u}$ and $\mathrm{u}$ ' wires have an angle of 30 degrees with respect to wires in $\mathrm{x}$ and $\mathrm{x}$ ' planes, while the v and v' have an angle of -30 degrees. Also the EDC is filled with Ar-Ethane 50/50.

A mechanical design of the EDC is shown in Fig. 2.19. The effective volume of the EDC is $12^{H} \times 100^{H} \times 30^{T} \mathrm{~cm}^{3}$.

The read-out electronics is designed to use the same 70 amplifier-discriminator cards (N277-L) as the HKS drift chamber (HDC), 35 each on the top and bottom sides.

The EDC geometrical/operational parameters are summarized in Table 2.5.

Table 2.5: EDC parameters.

\begin{tabular}{lc}
\hline \hline Geometrical parameters & \\
\hline Effective volume & $12^{H} \times 100^{W} \times 30^{T} \mathrm{~cm}^{3}$ \\
Wire plane configuration & $\mathrm{X}, \mathrm{X}^{\prime}, \mathrm{U}, \mathrm{U}^{\prime}, \mathrm{X}, \mathrm{X}, \mathrm{V}, \mathrm{V}, \mathrm{X}, \mathrm{X}$ \\
Operational parameters & \\
\hline Operation HV & $2200 \mathrm{~V}$ \\
Threshold & $2.0 \mathrm{~V}$ \\
Gas content & Ar-Ethane $50 / 50$ mixture \\
Gas pressure & $\sim 16 \mathrm{psi}$ \\
\hline \hline
\end{tabular}




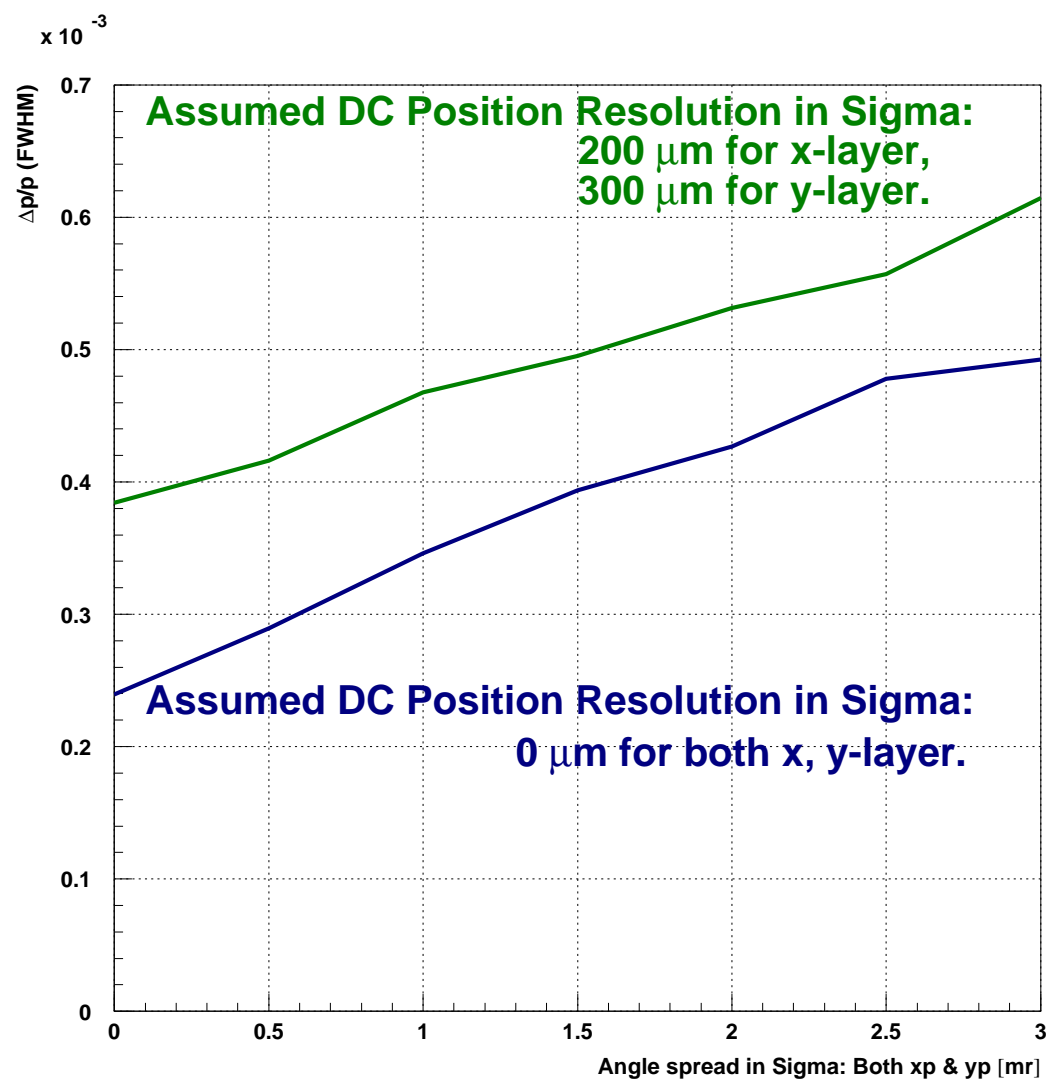

Figure 2.18: Multiple scattering angle dependence on $\Delta p$ of the Enge. Assuming local resolution of the Enge drift chamber $200 \mu \mathrm{m}(300 \mu \mathrm{m})$ for horizontal (vertical) plane, and angular spread in wire chamber less than $1.5 \mathrm{mr}$ for both horizontal and vertical plane, $\Delta p<5 \times 10^{-4}$ was expected by RAYTRACE. 


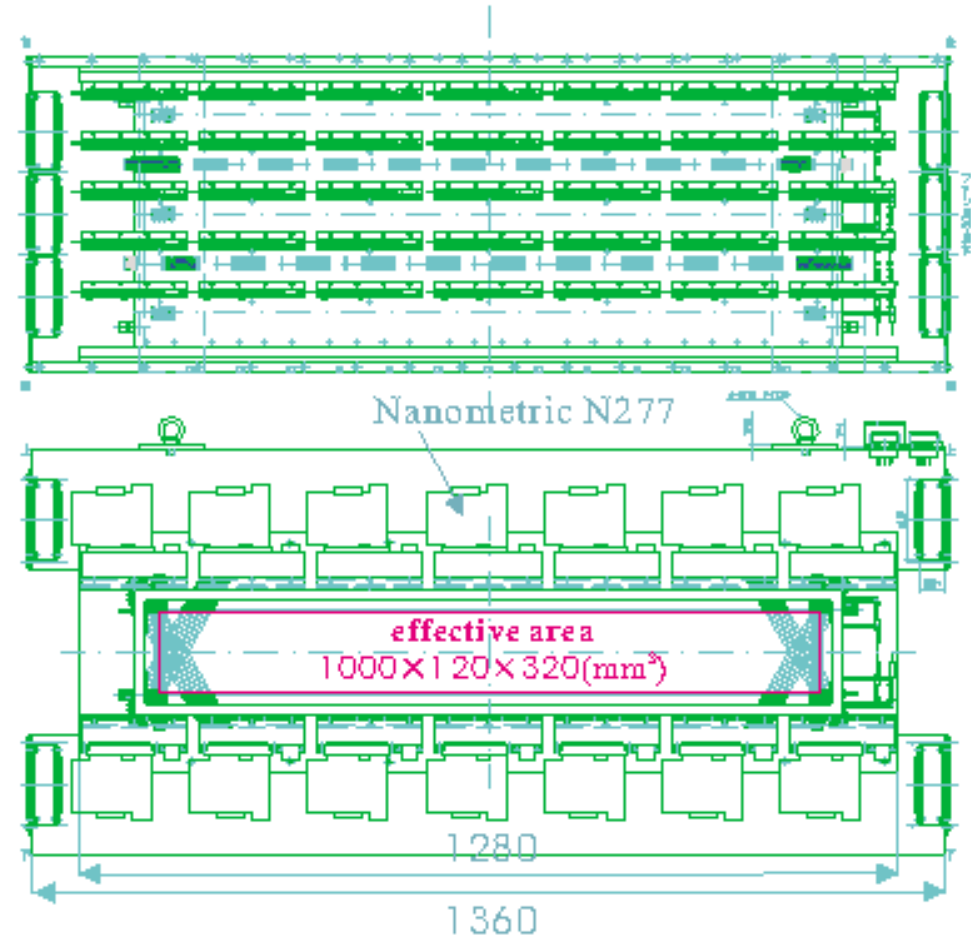

unit(mm)
whole wheight: $140 \mathrm{~kg}$

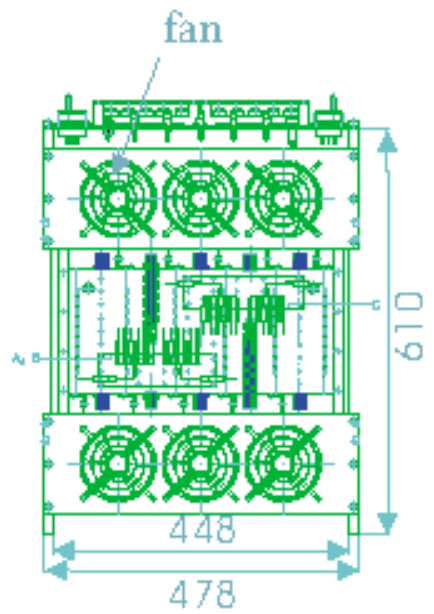

Figure 2.19: Mechanical drawing of the Enge Drift Chamber.

\section{Enge hodoscopes}

Two sets of scintillator hodoscopes are placed behind the EDC for trigger and timing measurement.

Fig. 2.20 shows a schematic view of the hodoscope. There are two identical sets of hodoscopes, EHODO1 and EHODO2, staggered with respect to each other by half the width of a segment. The hodoscope is designed assuming electron rate of at most $5 \mathrm{MHz}$. To keep the singles rate of each slat well below $1 \mathrm{MHz}$, the hodoscope is segmented into 25 plastic scintillator slats (Bicron BC420, $40^{W} \times 120^{L} \times 10^{T} \mathrm{~cm}^{3}$ ). Each of them is equipped with acrylic light guides and Hamamatsu H6612 3/4" phototubes on both ends.

\subsubsection{High resolution Kaon Spectrometer (HKS)}

The High resolution Kaon Spectrometer (HKS) consists of two quadrupole magnets (Q1 and Q2), one dipole (D) and a detector package. The configuration of the magnets is listed in Table 2.6. A detail description of the detector package follows.

\section{HKS drift chamber}

The two drift chambers (HDCs), which measure the particle tracks, are mounted directly on the HKS dipole magnet, $1 \mathrm{~m}$ apart from each other.

The momentum and outgoing angles at the target are reconstructed by transfer matrices which connect the HKS spectrometer's focal plane and target point. The particle position and angle at the focal plane are extracted from the measured particle tracks on the two HDCs.

Fig. 2.21 and 2.22 represent the layer configuration and geometrical layout of a HDC single plane. Each of the HDCs has 6 wire planes with multiple orientations in the plane transverse 


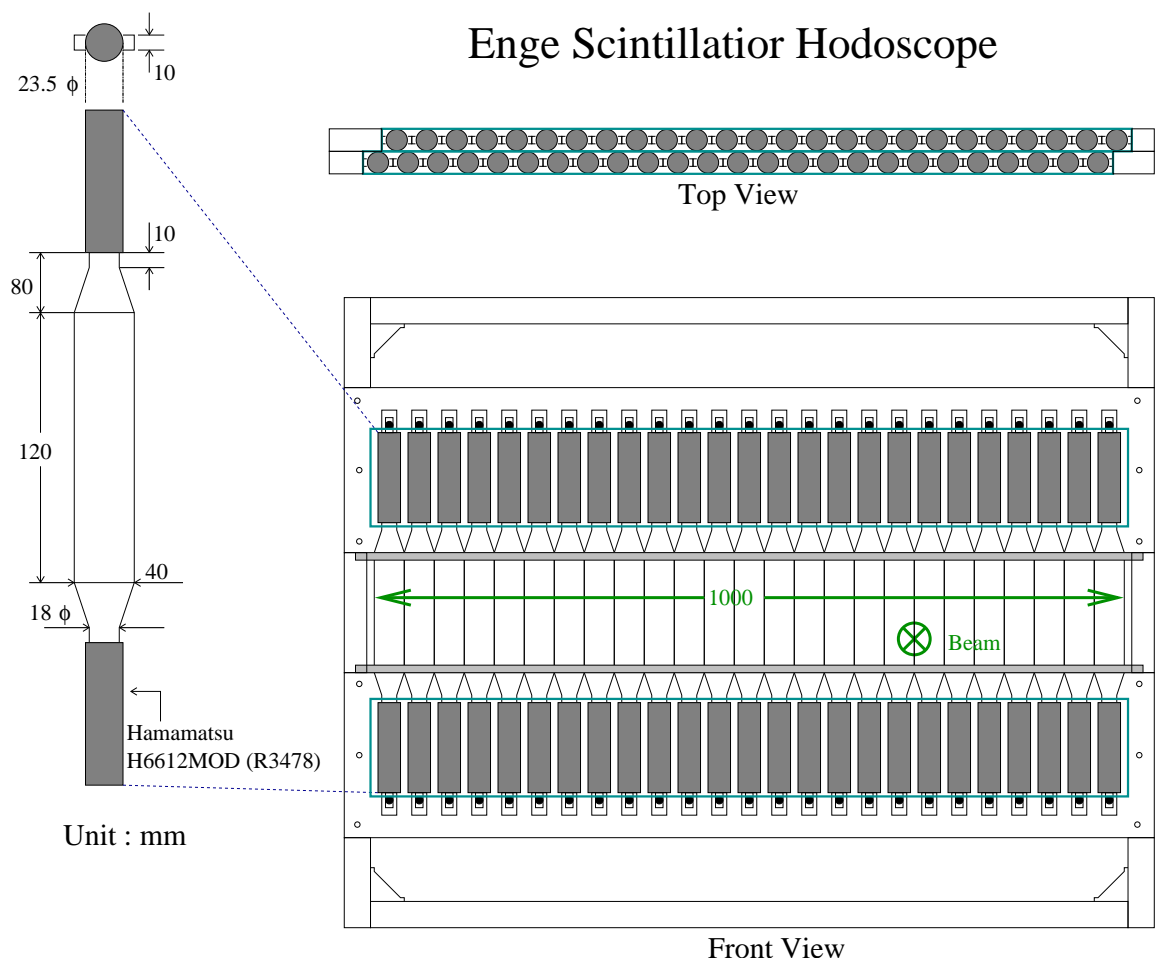

Figure 2.20: Schematic of Enge hodoscope.

Table 2.6: HKS magnet parameters

\begin{tabular}{|c|c|c|c|c|}
\hline Item & Q1 & Q2 & \multicolumn{2}{|c|}{$\overline{\mathrm{D}}$} \\
\hline Bore radius $[\mathrm{mm}]$ & 120 & 145 & \multicolumn{2}{|c|}{-} \\
\hline Pole gap height $[\mathrm{mm}]$ & - & - & \multicolumn{2}{|c|}{200} \\
\hline Pole length $[\mathrm{mm}]$ & - & - & \multicolumn{2}{|c|}{1560} \\
\hline Max. Ampere turns [A.turns] & 224000 & 144000 & \multicolumn{2}{|c|}{291840} \\
\hline Number of turns & 256 & 320 & \multicolumn{2}{|c|}{256} \\
\hline Conductor size $[\mathrm{mm}]$ & $8 \times 8$ ( $\phi 6$ hole $)$ & $13.5 \times 11.5(\phi 6.3$ hole $)$ & \multicolumn{2}{|c|}{$22 \times 22(\phi 12$ hole $)$} \\
\hline Field gradient $[\mathrm{T} / \mathrm{m}]$ & 6.6 & 4.2 & \multicolumn{2}{|c|}{-} \\
\hline Max. field $[\mathrm{T}]$ & - & - & \multicolumn{2}{|c|}{1.53} \\
\hline Max. current $[\mathrm{A}]$ & 875 & 450 & \multicolumn{2}{|c|}{1140} \\
\hline Resistance $[\mathrm{m} \Omega]$ & $181\left(@ 55^{\circ} \mathrm{C}\right)$ & $119\left(@ 45^{\circ} \mathrm{C}\right)$ & \multicolumn{2}{|c|}{$145\left(@ 47.5^{\circ} \mathrm{C}\right)$} \\
\hline & & & Gap side & Yoke side \\
\hline Cooling water flow rate $[l / \mathrm{min}]$ & 49.6 & 17.3 & 66.3 & 68.8 \\
\hline Pressure drop $[\mathrm{MPa}]$ & 0.36 & 0.38 & 0.32 & 0.35 \\
\hline Number of coolant circuits & 16 & 8 & 8 & 8 \\
\hline Total magnet weight [ton*] & 8.2 & 10.5 & \multicolumn{2}{|c|}{210} \\
\hline
\end{tabular}

* metric ton 
to the central trajectory of the particles. Wire orientations are perpendicular to the scattering angles (x, x'), and tilted by $30^{\circ}\left(\mathrm{u}, \mathrm{u}^{\prime}\right)$ and $-30^{\circ}\left(\mathrm{v}, \mathrm{v}^{\prime}\right)$ with respect to the $\mathrm{x}$ wires, respectively. Each plane has an effective area of $122 \times 30 \mathrm{~cm}^{2}$.

A pair of planes with the same orientation has an offset in half cell size of $0.5 \mathrm{~cm}$ from each other in order to increase the capability to resolve the left-right ambiguity. Each wire plane has a set of anode wires connected to ground and a set of potential wires and cathode foils set at negative high voltages. The wire material is gold plated tungsten and the cathode foils are made from $1 / 2$ mil of Mylar coated with 1,200 $\AA$ of $\mathrm{Cu}$ on each side.

The wires are separated by $1 \mathrm{~cm}$, and the maximum drift distance is $0.5 \mathrm{~cm}$. The HDCs are filled with Ar-Ethane 50/50. Each anode wire on each plane is directly connected to a channel of the 20 Nanometrics (N277-L) preamplifier card mounted on two sides of the chamber.

These cards amplify and discriminate the signals. Discriminated ECL signals are sent to a FASTBUS multihit TDC (F1 TDC). Up to 16 hits can be stored with a time resolution of 500 ps $[\sigma]$. See Table 2.7 for the detailed HDC parameters.
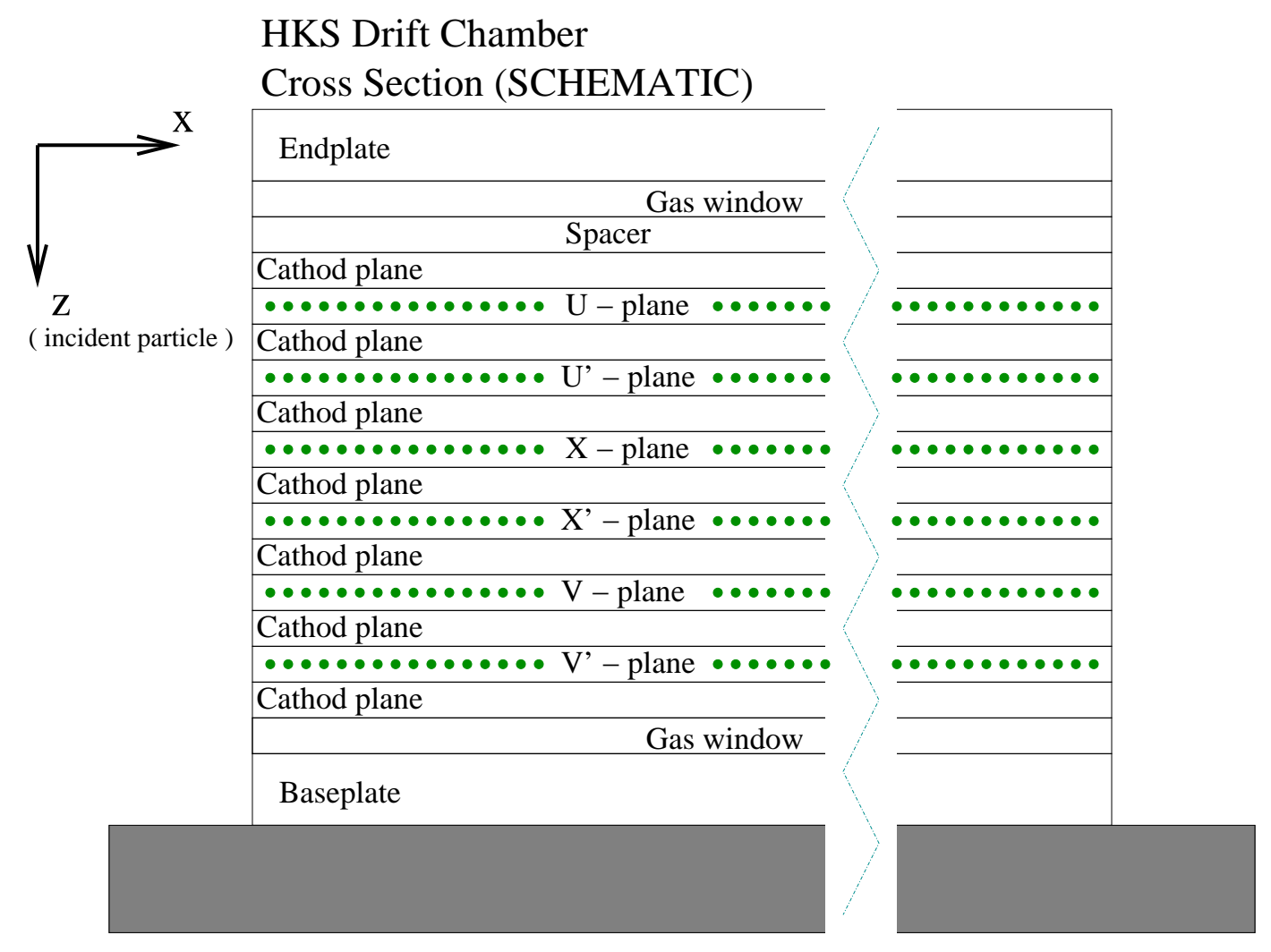

Figure 2.21: Layer configuration of the HKS drift chamber.

\section{HKS Time Of Flight (TOF) counters}

The HKS TOF counters determine the trigger timing and measure the velocity of the charged particles.

The HKS TOF counters consist of three sets of hodoscope layers, HTOF1X, HTOF1Y and HTOF2X and are made of Bicron BC408 scintillators and Hamamatsu H1949-50 phototubes on both ends.

Fig. 2.23 shows a schematic drawing of HTOF1X, which consists of 17 segments of scintillators. HTOF2X, which is similar to HTOF1X, consists of 18 segments of scintillators. Fig. 2.24 shows 


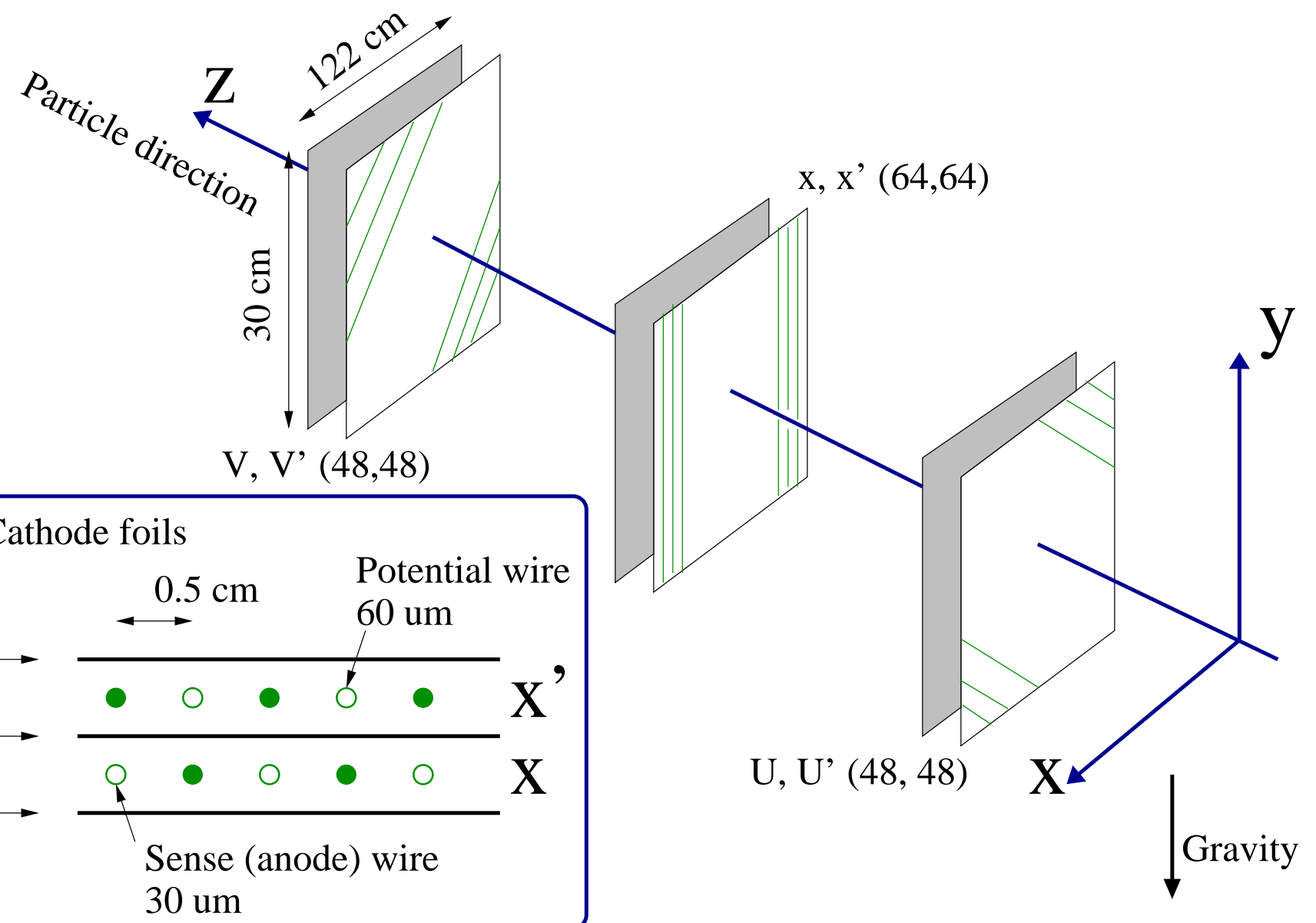

Figure 2.22: The geometry of the HKS drift chamber with the HKS coordinates.

Table 2.7: HDC parameters.

\begin{tabular}{lc}
\hline \hline Geometrical parameters & \\
\hline Effective region & $30^{H} \times 122^{W} \mathrm{~cm}^{2}$ \\
Wire plane configuration & $\mathrm{U}, \mathrm{U}^{\prime}, \mathrm{X}, \mathrm{X}, \mathrm{V}, \mathrm{V}$, \\
Operational parameters & \\
\hline Operation HV & $1970 \mathrm{~V}$ \\
Threshold & $3.0 \mathrm{~V}$ \\
Gas content & Ar-Ethane $50 / 50$ mixture \\
Gas pressure & $\sim 16 \mathrm{psi}$ \\
\hline \hline
\end{tabular}


a schematic of HTOF1Y which consists of 9 segments of scintillators.

See Table 2.8 for the detailed description of the HKS TOF counter configuration. HTOF1X and HTOF2X are placed $149.5 \mathrm{~cm}$ apart from each layer to separate kaons from pions and protons in the momentum region of interest.

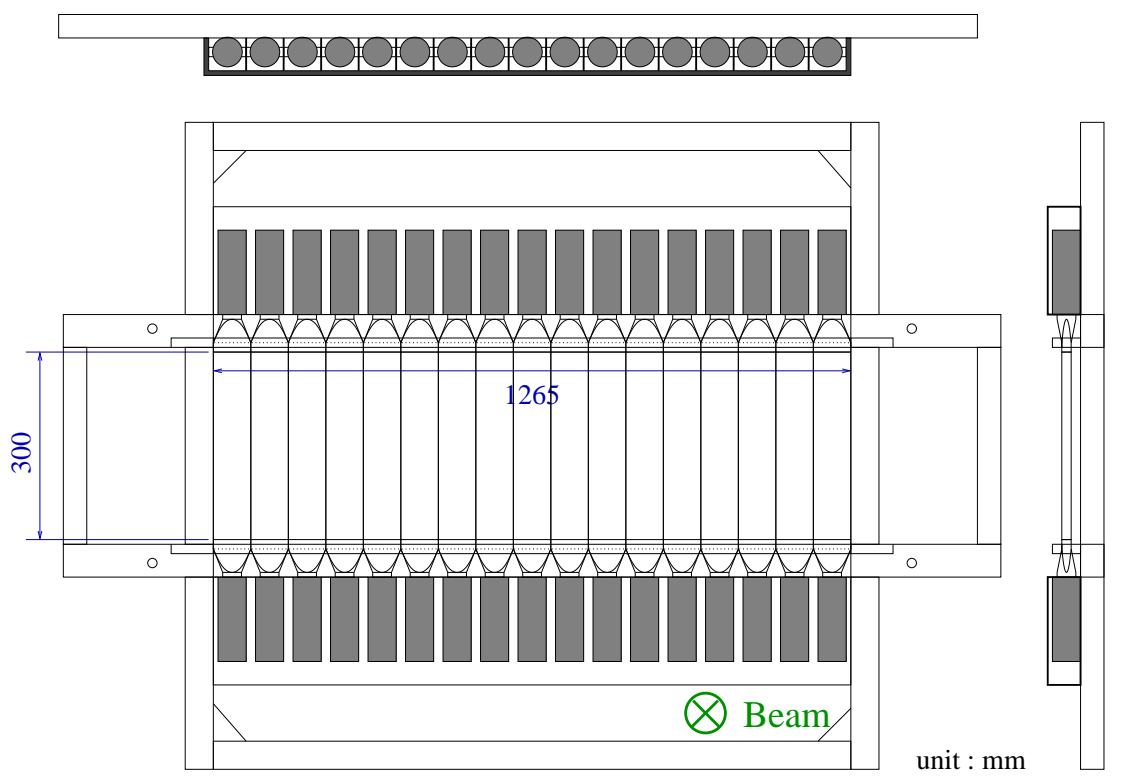

HKS hodoscope HTF1X

Figure 2.23: Schematic of the HTF1X.

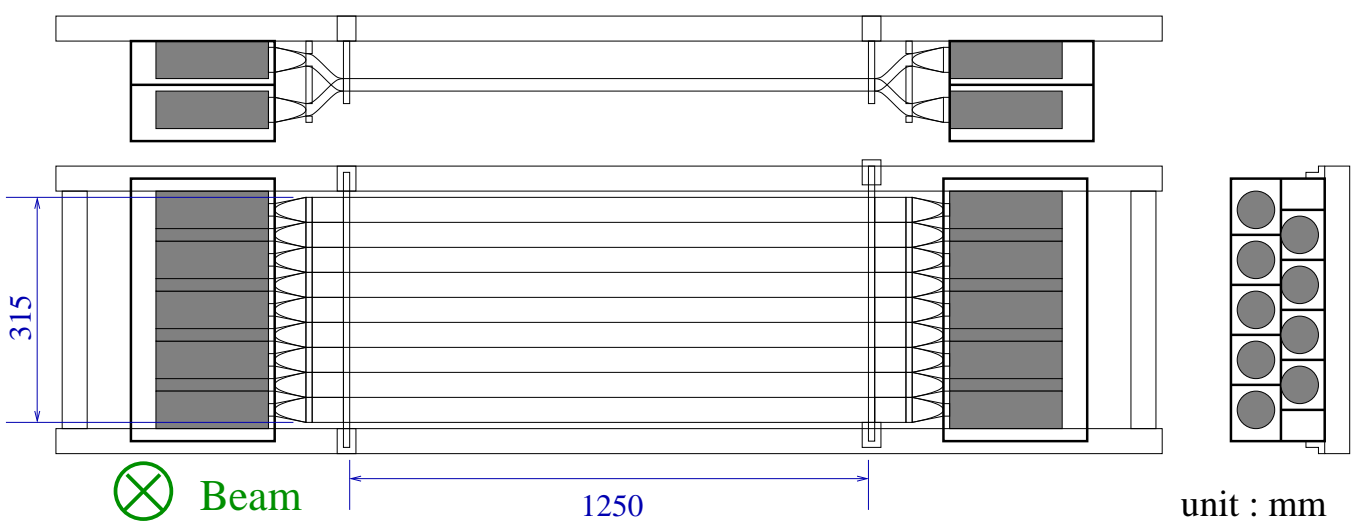

HKS hodoscope HTF1Y

Figure 2.24: Schematic of the HTF1Y.

\section{HKS aerogel Čerenkov counters}

The HKS aerogel Čerenkov counters (AČ) are mounted behind the HTOF1Y wall and used for pion suppression in the kaon trigger by a factor of $\sim 10^{-4}$.

AC consists of 7 segments $\times 3$ staggered layers in order to keep the individual PMT rates low and allow multiple tracks to be distinguished. The index of refraction of the silicon aerogel AČ radiators is 1.055, which can distinguish pion/kaon in a particle momentum region of 1.2 
Table 2.8: HTOF parameters.

\begin{tabular}{lcc}
\hline \hline Geometrical parameters & & \\
\hline HTOF1X & $30^{H} \times 125^{W} \times 2^{T} \mathrm{~cm}^{3}$ & $7.5^{W} \mathrm{~cm} \times 17$-segments, H1949-50 \\
HTOF1Y & $30^{H} \times 125^{W} \times 2^{T} \mathrm{~cm}^{3}$ & $3.5^{W} \mathrm{~cm} \times 9$-segments, H1949-50 \\
HTOF2X & $30^{H} \times 170^{W} \times 2^{T} \mathrm{~cm}^{3}$ & $7.5^{W} \mathrm{~cm} \times 18$-segments, H1949-50 \\
\hline Operational parameters & & \\
\hline Operation HV & $\sim-1800 \mathrm{~V}$ \\
\hline \hline
\end{tabular}

$\mathrm{GeV} / c \pm 12.5 \%$.

Fig. 2.25 represents a side and top view of the AČ. The size of one AČ layer is $46^{H} \times 169^{W} \times$ $31^{T} \mathrm{~cm}^{3}$, and the 7 segments in side the diffusion box are separated optically by Millipore memblanes (HAHY00010). Two different types of photopubes, Hamamatsu R1250 (28 for the 1st and 2nd layers) and Photonis XP4572B/D1 (14 for the third layer) are mounted on both ends.

Both tube types are operated in a cathode ground scheme (positive HV to anode). The R1250 used voltage dividers built at Florida International University (FIU), the XP4572B/D1 used the Photonis VD305 voltage divider with an additional amplifier built at JLab added directly into the divider housing.

In each segment, 5 layers of 4 by 2 silica aerogel tiles (Matsushita Electric Works; SP-50, $113^{H} \times$ $113^{W} \times 10^{T} \mathrm{~mm}^{3}$ for 1 tile) cover an area of $46 \times 23 \mathrm{~cm}^{2}$. The AC tile configuration is designed based on a test experiment in KEK-PS held on December 2002 (T530) [63].

HKS aerogel Cherenkov Counter

Side view
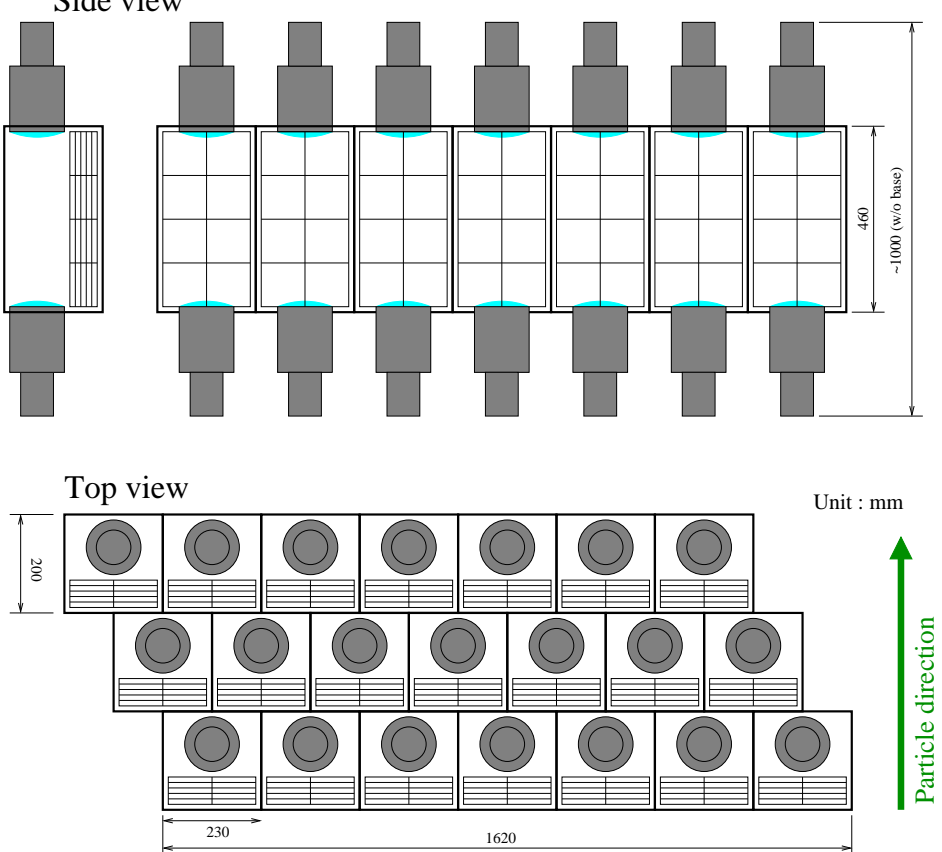

Figure 2.25: HKS aerogel Čerenkov counter. 


\section{HKS water Čerenkov counters}

The HKS water Čerenkov counters (WČ) are mounted behind the three layers of AČ and are used for proton suppression in the kaon trigger by a factor of $\sim 10^{-4}$.

The WČ consists of 12 segments $\times 2$ staggered layers in order to keep the individual PMT rate low and to distinguish between multiple tracks.

Details for the WČ are descibed in Chapter 3.

\subsection{Expected singles rate and energy resolution}

Singles particle rates in the Enge and HKS with $30 \mu \mathrm{A}$ of beam current are calculated and summarized in Table 2.9. The pion and proton rates in HKS are calculated based on the program EPC [64], and they are normalized by the experimental values measured in E89-009 for a $22 \mathrm{mg} / \mathrm{cm}^{2}$ of carbon target at $2.2^{\circ}$ detection angle.

The Quasi-free kaon production cross section is assumed to scale as $A^{0.8}$ which takes into account the effective proton numbers of the ${ }^{12} \mathrm{C}\left(\gamma, \mathrm{K}^{+}\right)$reaction [65].

The positron rate in the HKS is evaluated with a GEANT calculation using $1.7 \times 10^{4}$ positron events (corresponds to $1.9 \times 10^{9}$ beam electrons bombarding $100 \mathrm{mg} / \mathrm{cm}^{2}$ carbon target) generated by the EGS code. No positrons passed through the dipole.

The electron rate in the Enge is evaluated and compared with the EGS code and Lightbody's code, which agreed more or less to each other.

The pion rate in the Enge is calculated based on the EPC code, and normalized by the same factor used for the hadron rates in HKS.

As shown in Table 2.9, the singles rate in the HKS is dominated by positive pions, while for the Enge by electrons. The positive pions and protons in HKS are rejected in the trigger by the aerogel and water Čerenkov counters up to $\sim 1 \mathrm{MHz}$ for positive pion and $\sim 0.5 \mathrm{MHz}$ for proton, respectively. Rejection efficiencies for the aerogel and water Cerenkov counters are estimated as $1 \times 10^{-4}$ and $5 \times 10^{-4}$, respectively.

Singles rates of background electrons, i.e. Bremsstrahlung electrons and Møller scattering electrons are reduced with the tilt method to be less than $5 \mathrm{MHz}$.

Table 2.9: Calculated singles rates. Detection angle of $\theta_{\mathrm{e}},=4.5^{\circ}, \theta_{\mathrm{K}^{+}}=7^{\circ}$ for scattering electrons and kaons respectively, $100 \mathrm{mg} / \mathrm{cm}^{2}$ target thickness and a $30 \mu \mathrm{A}$ beam current assumed.

\begin{tabular}{c|cccc|cc}
\hline \hline \multirow{3}{*}{ Target } & \multicolumn{4}{|c|}{ HKS } & \multicolumn{2}{|c}{ Enge } \\
& $\mathrm{e}^{+}$ & $\pi^{+}$ & $\mathrm{K}^{+}$ & $\mathrm{p}$ & $\mathrm{e}^{-}$ & $\pi^{-}$ \\
& {$[\mathrm{kHz}]$} & {$[\mathrm{kHz}]$} & {$[\mathrm{kHz}]$} & {$[\mathrm{kHz}]$} & {$[\mathrm{kHz}]$} & {$[\mathrm{kHz}]$} \\
\hline${ }^{12} \mathrm{C}$ & - & 420 & 0.38 & 150 & 1,000 & 2.8 \\
${ }^{28} \mathrm{Si}$ & - & 420 & 0.32 & 130 & 1,900 & 2.8 \\
\hline \hline
\end{tabular}

The expected energy resolution of bound states in the ${ }_{\Lambda}^{12} \mathrm{~B}$ and ${ }_{\Lambda}^{28} \mathrm{Al}$ mass spectra are shown in Table 2.10. The beam energy is monitored with a precision of $10^{-4}$. The Enge and HKS momentum resolutions, which involve multiple scattering effects on the drift chambers, are evaluated with a RAYTRACE/GEANT calculation as $2 \times 10^{-4}$ and $5 \times 10^{-4}$, respectively. Kinematic broadening is proportional to the inverse of the target mass number and predominantly depends on the angular resolution of the HKS. The energy loss of incident/outgoing electrons and kaons at the target for various targets are calculated by SIMC [66] and summarized in Table 2.11. The energy loss at the target is taken into account in the momentum 
calibration.

Eventually, a resolution of $\sim 400 \mathrm{keV}$ (FWHM) is expected for each target.

Table 2.10: Expected energy resolution for bound states in ${ }_{\Lambda}^{7} \mathrm{He}$ and ${ }_{\Lambda}^{12} \mathrm{~B}$ mass spectra.

\begin{tabular}{c|cc}
\hline \hline Components & Resolution $[\mathrm{keV}](\mathrm{FWHM})$ \\
\hline Target & \multicolumn{2}{|c}{${ }^{7} \mathrm{Li}$} \\
\hline Beam momentum $\left(<1 \times 10^{-4}\right)$ & \multicolumn{2}{|c}{$<180$} \\
HKS momentum $\left(2 \times 10^{-4}\right)$ & \multicolumn{2}{|c}{230} \\
Enge momentum $\left(5 \times 10^{-4}\right)$ & \multicolumn{2}{|c}{120} \\
Kinematic broadening $\left(\Delta \theta_{\mathrm{K}^{+}}=2.9 \mathrm{mr}\right)$ & 132 & 78 \\
Target thickness $\left(100 \mathrm{mg} / \mathrm{cm}^{2}\right)$ & $<145$ & $<157$ \\
\hline Overall & $<370$ & $<360$ \\
\hline \hline
\end{tabular}

Table 2.11: Energy loss calculation at the target for incident and scattered electrons and kaon with various targets. $1.851 \mathrm{GeV}$ of electron beam energy is fixed and 0.316 and $1.200 \mathrm{GeV} / c$ of central momenta are assumed for scattered electron and kaon, respectively.

\begin{tabular}{c|ccc}
\hline \hline Target & \multicolumn{3}{|c}{ Energy loss [keV] } \\
& Incident electron & Outgoing electron & Kaon \\
\hline $\mathrm{CH}_{2}\left(465 \mathrm{mg} / \mathrm{cm}^{2}\right)$ & 492 & 601 & 489 \\
${ }^{7} \mathrm{Li}\left(189 \mathrm{mg} / \mathrm{cm}^{2}\right)$ & 145 & 178 & 150 \\
${ }^{12} \mathrm{C}\left(100 \mathrm{mg} / \mathrm{cm}^{2}\right)$ & 82 & 102 & 86 \\
\hline
\end{tabular}

\subsection{Trigger}

\subsubsection{Grouping trigger}

The main trigger is constructed by the HKS detector package.

The Enge detector package, which consists of EDC and two layors of EHODO, is not included in the electronic trigger because of its high counting rate. A coincidence between the two arms (HKS and Enge) is created by the offline analysis as discussed in 4.4 A simple idea of the kaon trigger is described as follows :

$$
\mathrm{K}^{+} \text {trigger }=(\mathrm{HTOF}) \otimes(\overline{\mathrm{AC}}) \otimes(\mathrm{W} \check{\mathrm{C}})
$$

where HTOF, AČ and WČ are described with $\oplus$ or $\otimes$ between each layer as follows.

$$
\begin{aligned}
\mathrm{HTOF} & =\mathrm{HTOF} 1 \mathrm{X} \otimes \mathrm{HTOF} 2 \mathrm{X} \\
\mathrm{AC} & =\mathrm{AC} 1 \oplus \mathrm{AC} 2 \oplus \mathrm{AC} 3 \\
\mathrm{WC} & =\mathrm{WC} 1 \otimes \mathrm{WC} 2
\end{aligned}
$$

As already discussed in 2.4, the singles rates of pions and protons are expected to be very high. Therefore, we introduced a grouping trigger to prepare for the kaon overkilling issue. 
According to a Monte Carlo simulation, which indicates that there are several strong bunches of focus points on the HKS dispersion plane, the detector package is partitioned into 6 groups as shown in Fig. 2.26.

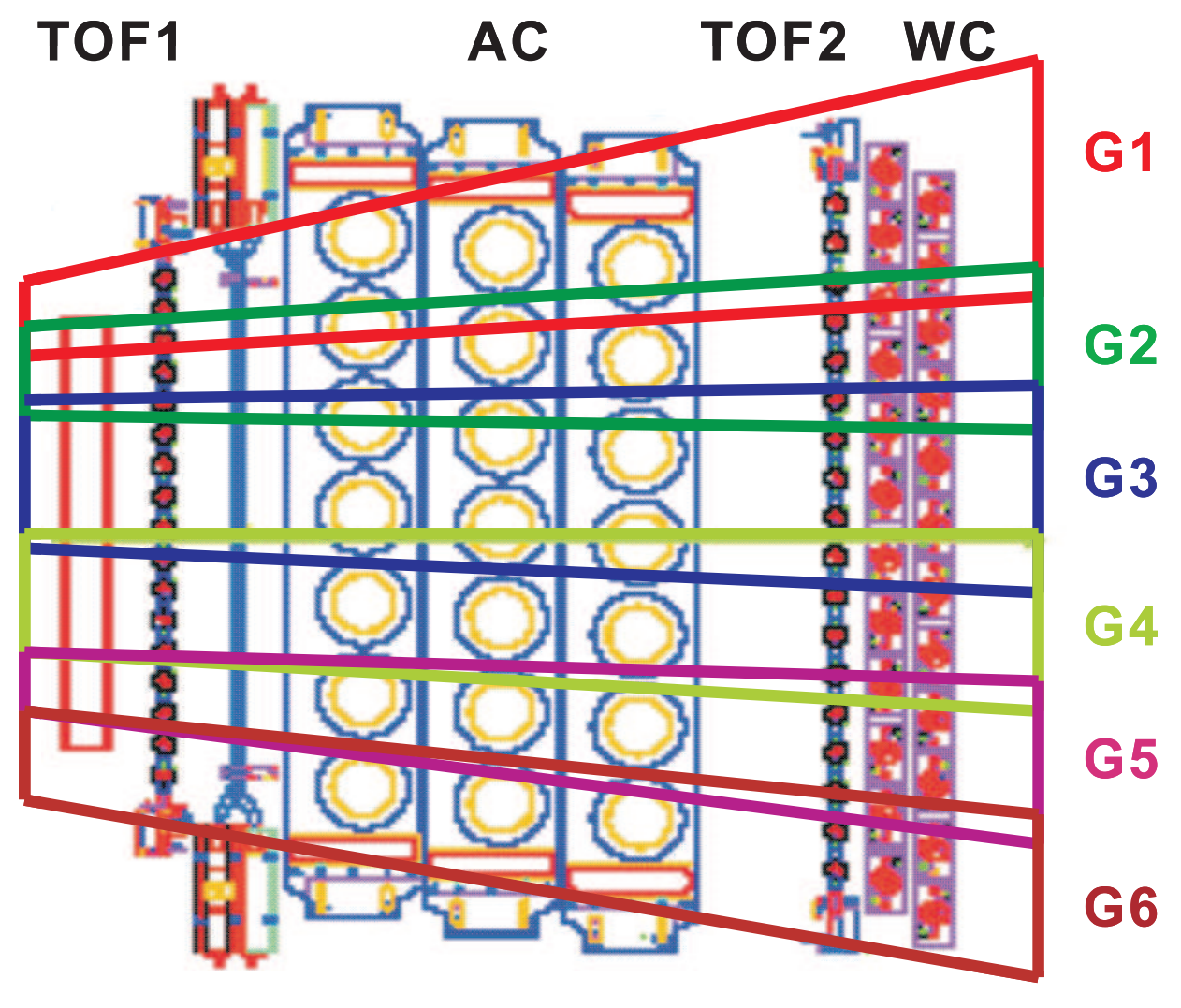

Figure 2.26: Schematics of a grouping trigger for HKS detector package.

In addition, by constructing a trigger for each group, the kaon overkilling can be avoided because the real singles rate in each group is decreased compared to the case of no grouping trigger. Also, the momentum range of particles passing through each group can be roughly determined because the grouping is segmented along the dispersion plane. Therefore, by setting a proper threshold for the Čerenkov counters, kaon candidates can be effectively selected.

As a reference, the grouping definition for the real event distribution on the HTOF1X and 2X planes is represented in Fig. 2.27, and shows an availability of the grouping trigger introduction.

\subsubsection{Tohoku Universal Logic module (TUL-8040)}

Constructing trigger logic group by group with conventional NIM (Nuclear Instruments Modules) logics would require a vast numbers of modules and cables.

In order to avoid this, we developed and introduced a programable logic module, the so called Tohoku Universal Logic module (TUL-8040).

The TUL-8040 has functions which are equivalent to a few tens of NIM modules, and can be configured by changing programs.

Furthermore, the propagation time in one TUL-8040 module is less than 50 ns.

The TUL-8040 uses the ALTERA APEX 20K series of FPGA (Field Programable Gate Array). 


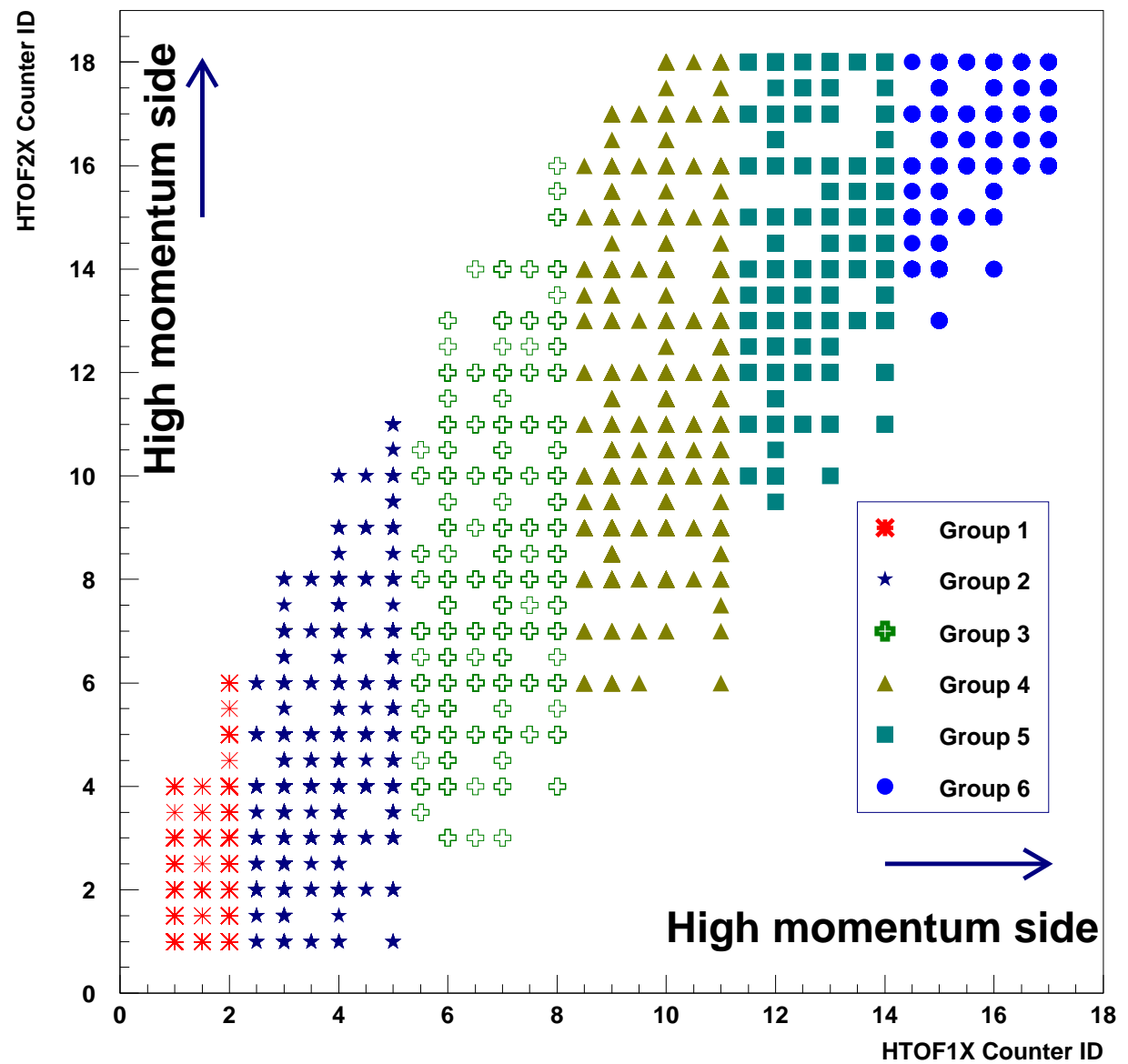

Figure 2.27: Grouping definition for real event distribution on HTOF1X and 2X. 
An FPGA is an extensive programable logic device, consists of various numbers of logic gates and flip-flop elements in arrays with programable connections. This series of FPGA has already been utilized in several nuclear physics experiments [67,68].

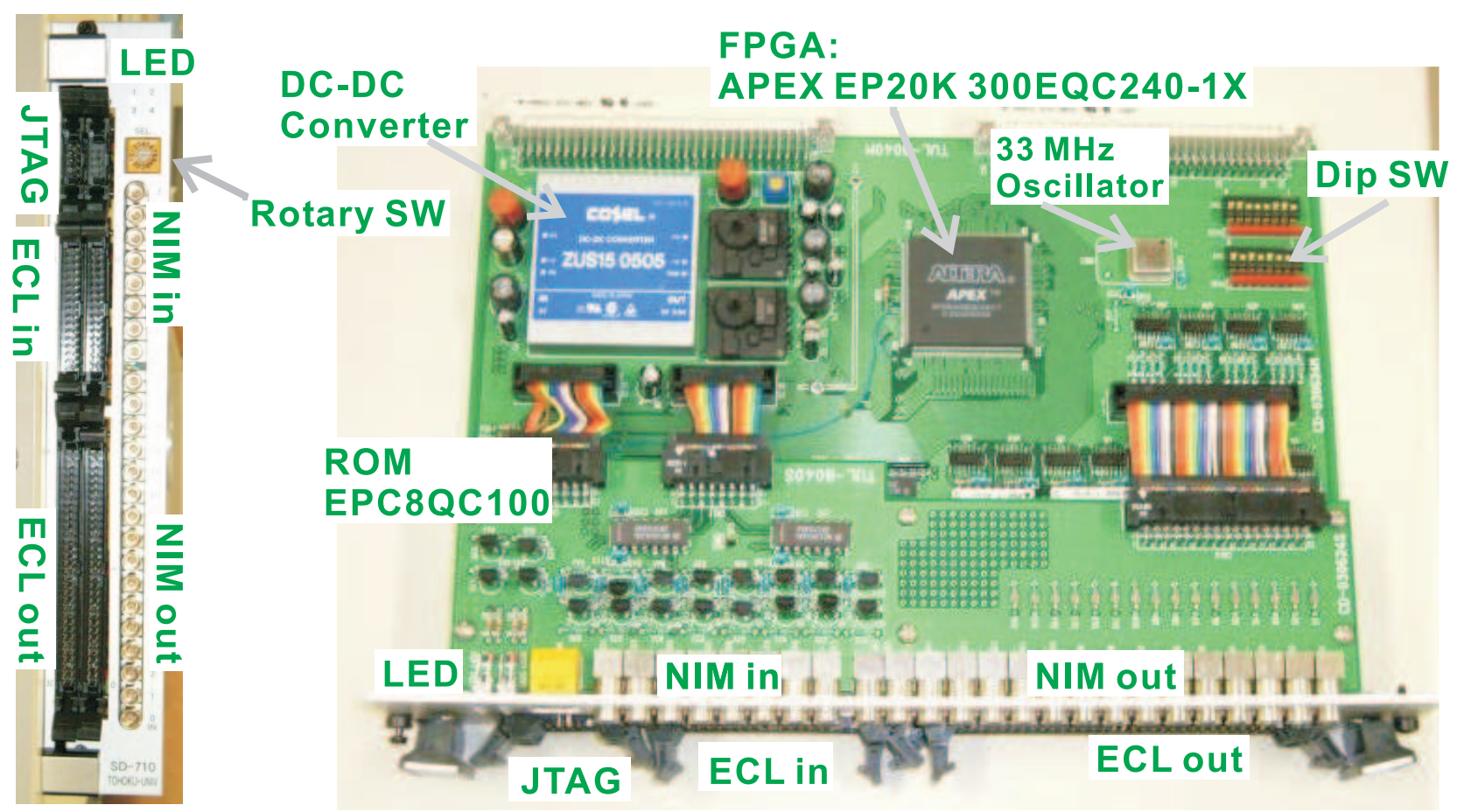

Figure 2.28: Front (left) and top (right) view of the TUL-8040.

The design of TUL-8040 is summarized as follows,

- VME $6 \mathrm{U} 2$ width

- 80 ch inputs (ECL / LVDS 64 ch + NIM 16 ch)

- 40 ch outputs (ECL 32 ch + NIM 8 ch)

- 16 bit dip switch

- 4 bit rotary switch

- 4 LEDs

and schematic view of the TUL-8040 is shown in Fig. 2.28.

The ALTERA Quartus II is used for HDL programing for the FPGA. The program cab be downloaded with JTAG from a computer to two types of storage device, SRAM and ROM on the TUL-8040. SRAM is specified for temporary storage for debug use because SRAM is lost or erased at poweroff. The HDL program stored in ROM is loaded to the FPGA at poweron, therefore the final or stable version of the HDL program should be stored in ROM.

Details of design and performance is described in [69].

In total 6 TUL-8040 modules were installed for the 6 counters; HTOF1X, HTOF2X, HTOF1Y, AČ, WČ and ENGE hodoscopes.

For each counter, the TUL-8040 makes . AND . of PMT signals from both end, then divides them 
to each group defined on each counter layer.

\subsubsection{Trigger}

The main part of the trigger scheme is illustrated in Fig. 2.29. The trigger timing is determined by the HTOF1X and HTOF2X. .OR. of two PMT signals from both end of HTOF1X and HTOF2X are collected to create pretrigger. Grouped 6 signals by TUL-8040 from the aerogel, water Čerenkov counters and HTOF counters are divided and used for particle identification, and collected to create HKS pretrigger. The signals are collected and discriminated by a reasonable threshold to be sent to TDC and scalers.
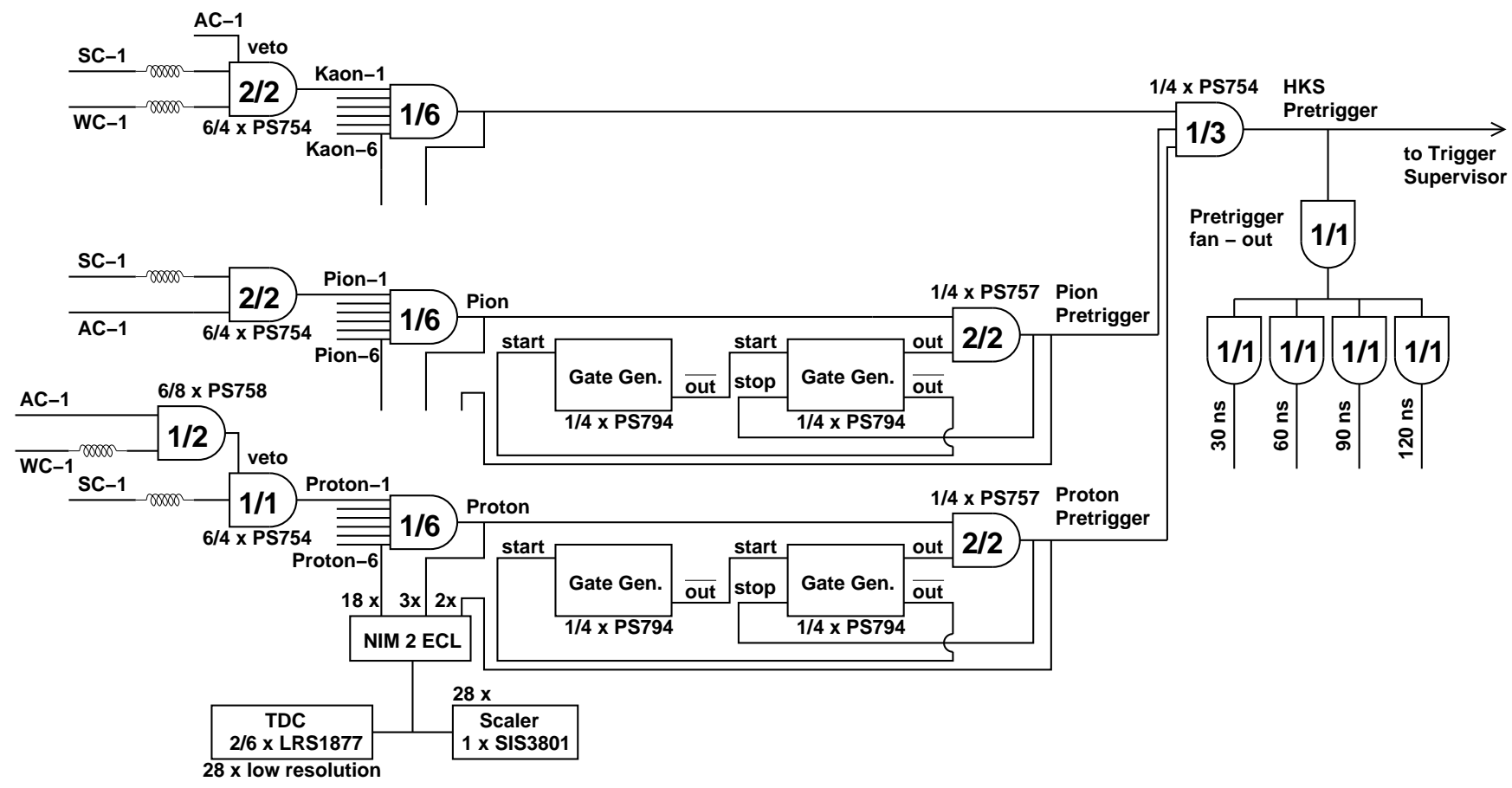

Figure 2.29: The HKS trigger logic.

All triggers are sent to a programable logic module called the 8LM (LeCroy 2365) and followed by the Trigger Spervisor (TS) which has been developed and built at JLab.

The TS logic diagram is described in Fig. 2.30. The 8LM receives TS BUSY, TS EN1 or TS $G O$ signals and pretriggers. The meanings of each signal are follows:

TS GO : $\quad$ Run has started,

TS EN1: $\quad$ Run has started and data taking is enabled,

TS BUSY: $\quad$ Run has started but data taking is disabled.

The 8LM produces TRIG and PRETRIG signals, the only difference being whether the TS $B U S Y$ signal is affected $(T R I G)$ or not (PRETRIG). All outputs in the 8LM are sent to the scalers and TDCs to be recorded. The 8LM sends the selected TRIG signals to the TS, producing a pair long gates (more than $100 \mu \mathrm{s}$ ). All the ADC and TDC gates are generated by coincidence between the selected TRIG signals and the long gate. 


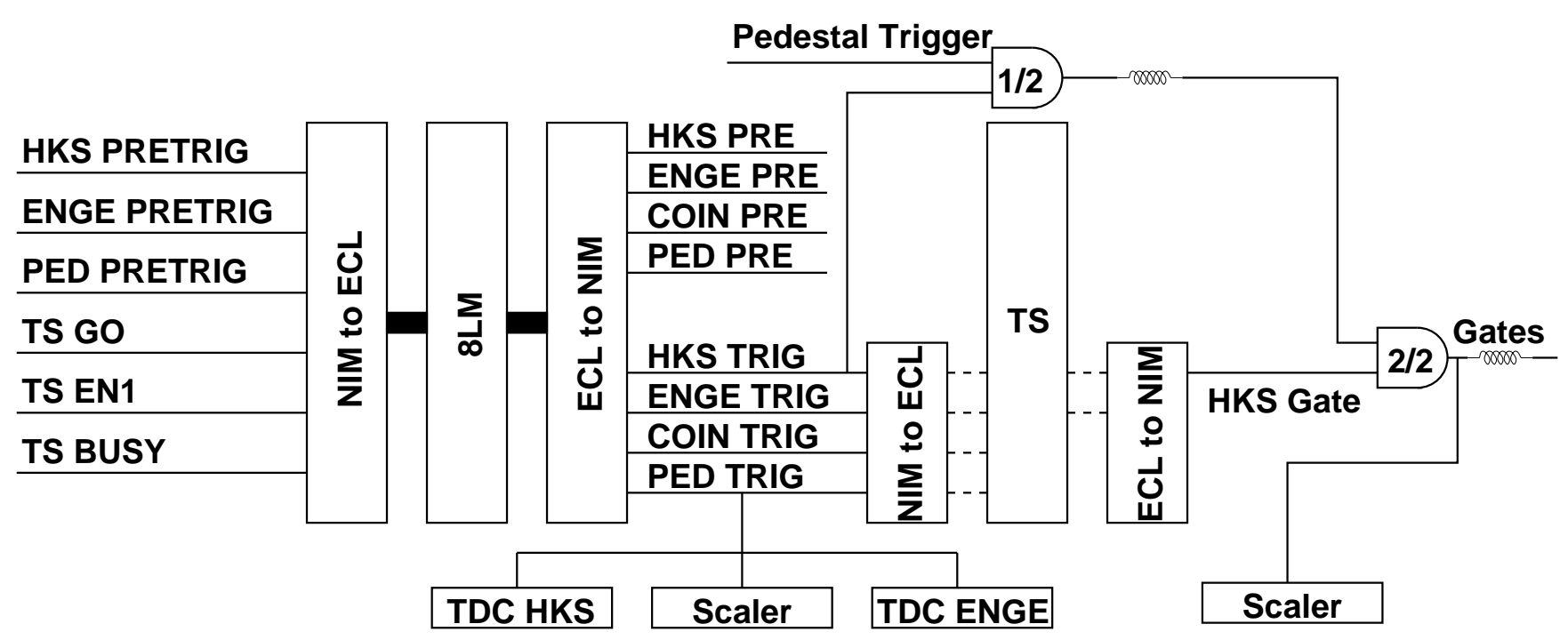

Figure 2.30: Trigger Supervisor (TS) logic.

\subsection{Data acquisition}

The digitized electronic signals were converted to physics data sets written on disks by the CEBAF On-line Data Acquisition (CODA) system [70]. General components which comprise the CODA system are described in Fig. 2.31.

All signals from PMTs are sent to the counting house and digitized by FASTBUS TDCs and ADCs. Scaler information is recorded by VME scalers. Each front-end crate is managed by a Read Out Controller (ROC) processor.

The Trigger Supervisor (TS) is a central control point for acquisition activities. The role of the TS is summarized as follows.

1 Accepts and prescales multiple sources of triggers.

2 Maintains system busy while an input trigger is being processing.

3 Generates signals used for the gating and timing of front-end modules (TDCs and ADCs).

4 Coordinates the actions of multilevel trigger systems.

5 Communicates triggering information to the system's read out controllers.

6 Keeps track of the number of events currently in the buffers of the front-end modules.

The ROCs read the event data and sent event fragments to the Event Builder (EB) which runs on the host computer.

Combined data fragments from the EB are buffered into Event Transfer (ET) system. The ET system is not the bottle neck or limit to DAQ rate. We are limited by the convertion time of the front-end modules, mostly the FASTBUS 1875A TDCs used for PMTs. As event rate is higher, event size which DAQ process is decreased, and event rate (coincidence of ENGE and HKS triggers) which is sent to the ET is required to be reduced. Therefore we introduced the Cerenkov counters and grouping triggers in order to suppress the coincidence trigger rate to be less $1 \mathrm{MHz}$. Typical ENGE, HKS and coincidence trigger rate for various targets and currents are summarized in Table 2.12. Efficiency of this DAQ data process is estimated as a computer living time efficiency as discussed in Chapter 4. The ET distributes data to online monitoring 


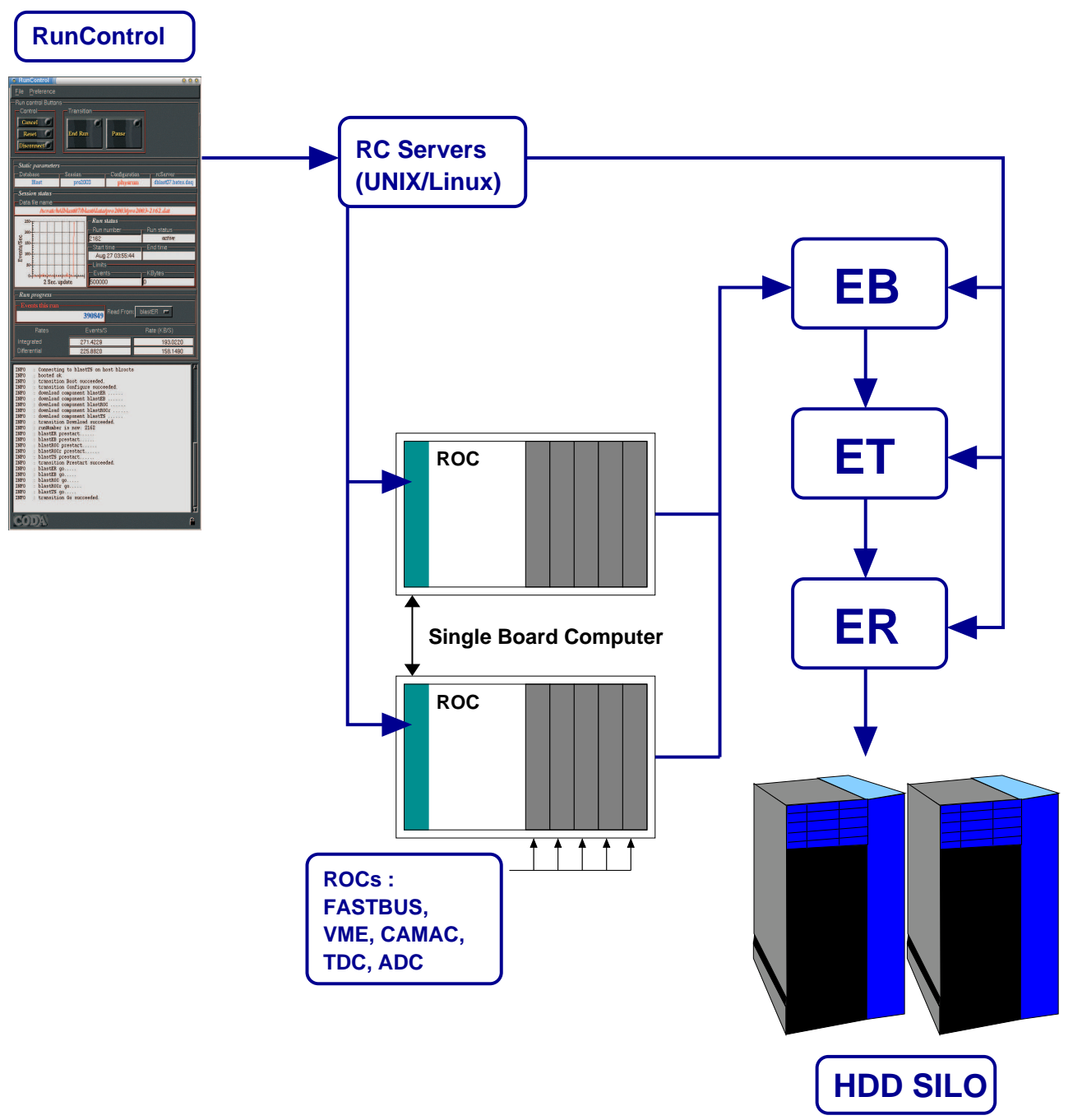

Figure 2.31: A flowchart of CODA. 
Table 2.12: Typical ENGE, HKS and Coincidence trigger rate.

\begin{tabular}{lcccc}
\hline \hline Target & Current $[\mu \mathrm{A}]$ & HKS $[\mathrm{kHz}]$ & ENGE $[\mathrm{MHz}]$ & Coin. $[\mathrm{kHz}]$ \\
\hline${ }^{6} \mathrm{Li}$ & 16.0 & 8.1 & 1.0 & 0.29 \\
& 20.0 & 12.2 & 1.2 & 0.55 \\
& 25.0 & 20.3 & 1.5 & 1.09 \\
\hline${ }^{7} \mathrm{Li}$ & 5.0 & 1.0 & 0.3 & 0.01 \\
& 25.0 & 13.0 & 1.2 & 0.69 \\
& 28.0 & 13.8 & 1.3 & 0.71 \\
\hline${ }^{9} \mathrm{Be}$ & 19.0 & 10.1 & 0.9 & 0.40 \\
& 20.0 & 13.5 & 1.1 & 0.56 \\
& 23.7 & 17.3 & 1.3 & 0.79 \\
\hline${ }^{10} \mathrm{~B}$ & 8.0 & 1.5 & 0.4 & 0.02 \\
& 16.0 & 4.3 & 0.7 & 0.12 \\
& 30.0 & 14.2 & 1.3 & 0.69 \\
\hline${ }^{12} \mathrm{C}$ & 13.0 & 3.5 & 0.6 & 0.09 \\
& 26.0 & 10.1 & 1.2 & 0.46 \\
& 30.0 & 14.8 & 1.4 & 0.73 \\
\hline${ }^{28} \mathrm{Si}$ & 7.0 & 2.8 & 0.6 & 0.55 \\
& 13.0 & 8.4 & 1.2 & 0.36 \\
& 18.0 & 16.4 & 1.7 & 0.96 \\
\hline${ }^{51} \mathrm{~V}$ & 14.0 & 7.5 & 1.3 & 0.40 \\
& 18.0 & 15.4 & 1.8 & 1.04 \\
\hline${ }^{89} \mathrm{Y}$ & 10.0 & 10.0 & 1.4 & 0.52 \\
& 13.0 & 17.5 & 1.8 & 1.10 \\
\hline${ }^{208} \mathrm{~Pb}$ & 0.3 & 9.9 & 1.0 & 0.38 \\
& 0.4 & 9.6 & 1.0 & 0.36 \\
\hline \hline
\end{tabular}


system accoding to user's requirements, and sends data to the Event Recorder (ER). Finally the ER controls events to the disks.

GB ethernet interfaces connect the various CODA components, and the user controlls the data acquisition through a GUI interface.

At the beginning of each run, a pedestal trigger is applied to determine the value and width of all ADCs to pedestals.

The physics data are stored until the maximum data size set is reached (1 Gbyte/run). Scaler data are stored in every 2 seconds during data taking. Informations such as spectrometer settings, accelerator and target status are read out from the CEBAF EPICS database by a script at periods of $2 \sim 30$ seconds. 


\subsection{Data summary}

Data were taken for a total of ten targets as listed in Table 2.13. $\mathrm{A} \mathrm{CH}_{2}$ target was used for mass scale calibration. Data taking for the ${ }^{208} \mathrm{~Pb}$ target was used for a quasi-free study as demonstrartion for a future experiment. Table 2.13 shows a summary of E01-011 data taking. During the commissioning run $(\# 1 \sim \# 4)$ particle rates on each counter were investigated and the grouping trigger was found not to be necessary. For the production run $(\# 5 \sim \# 22)$, trigger settings of Čerenkov counters were changed frequently for rate and trigger study, except \#8. HTOF1Y was introduced from \#12.

Table 2.13: Data acquisition settings. Here, $\mathrm{T} \oplus \mathrm{B}(\mathrm{T} \otimes \mathrm{B})$ represents a .OR. (.AND.) logic of two PMT mounted on Top/Bottom side of each Cerenkov counter.

\begin{tabular}{|c|c|c|c|c|c|}
\hline $\begin{array}{c}\text { Data set } \\
\text { No. }\end{array}$ & $\begin{array}{c}\text { Beam current } \\
\text { (Ave.) }[\mu \mathrm{A}]\end{array}$ & Target & Grouping & $\begin{array}{c}\text { Trigger } \\
\mathrm{ENGE} \times \mathrm{HTOF} 1 \mathrm{X} \times \mathrm{HTOF} 2 \mathrm{X} \times \alpha\end{array}$ & $\begin{array}{c}\text { Beam charge } \\
{[\mathrm{mC}]}\end{array}$ \\
\hline 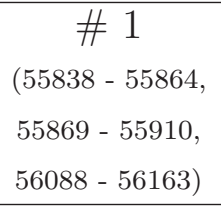 & 2.3 & $\mathrm{CH}_{2}$ & $\mathrm{OFF}$ & 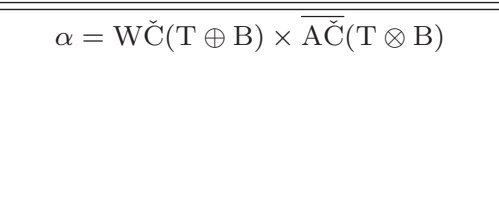 & 247.2 \\
\hline $\begin{array}{c}\# 2 \\
(55911-55921, \\
56167-56228)\end{array}$ & 16.1 & ${ }^{12} \mathrm{C}$ & OFF & $\alpha=\mathrm{WC}(\mathrm{T} \oplus \mathrm{B}) \times \overline{\mathrm{AC}}(\mathrm{T} \otimes \mathrm{B})$ & 618.5 \\
\hline $\begin{array}{c}\# 4 \\
(56231-56282) \\
\end{array}$ & 19.7 & ${ }^{12} \mathrm{C}$ & OFF & $\alpha=\mathrm{WC}(\mathrm{T} \otimes \mathrm{B}) \times \overline{\mathrm{AC}}(\mathrm{T} \oplus \mathrm{B})$ & 249.8 \\
\hline $\begin{array}{c}\#=5 \\
(56283-56352, \\
56629-56663, \\
57203-57224, \\
57318-57334)\end{array}$ & 2.3 & $\mathrm{CH}_{2}$ & $\mathrm{ON}$ & $\alpha=\mathrm{WC}(\mathrm{T} \otimes \mathrm{B}) \times \overline{\mathrm{AC}}(\mathrm{T} \oplus \mathrm{B})$ & 431.8 \\
\hline $\begin{array}{c}\# \text { \# } \\
(56353-56474, \\
56528-56628, \\
56665-57200)\end{array}$ & 24.4 & ${ }^{12} \mathrm{C}$ & $\mathrm{ON}$ & $\alpha=\mathrm{W} \breve{\mathrm{C}}(\mathrm{T} \otimes \mathrm{B}) \times \overline{\mathrm{AC}}(\mathrm{T} \oplus \mathrm{B})$ & 6687.3 \\
\hline $\begin{array}{c}\# 7 \\
(56476-56523, \\
57225-57247, \\
57270-57317, \\
57335-57476, \\
57482-57528)\end{array}$ & 19.6 & ${ }^{9} \mathrm{Be}$ & $\mathrm{ON}$ & 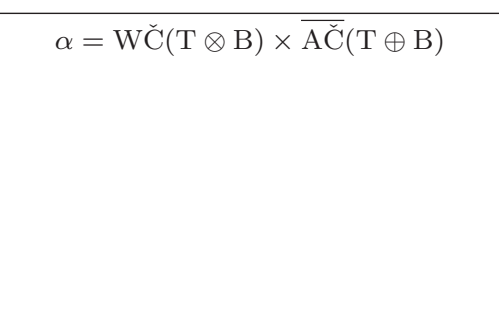 & 2367.5 \\
\hline $\begin{array}{c}\# 11 \\
(57547-57645, \\
57683-57727)\end{array}$ & 21.3 & ${ }^{12} \mathrm{C}$ & $\mathrm{ON}$ & $\alpha=\mathrm{WC}(\mathrm{T} \otimes \mathrm{B}) \times \overline{\mathrm{AC}}(\mathrm{T} \otimes \mathrm{B})$ & 1129.7 \\
\hline
\end{tabular}




\begin{tabular}{|c|c|c|c|c|c|}
\hline $\begin{array}{c}\text { Data set } \\
\text { No. } \\
\end{array}$ & $\begin{array}{c}\text { Beam current } \\
\text { (Ave.) }[\mu \mathrm{A}]\end{array}$ & Target & Grouping & $\begin{array}{c}\text { Trigger } \\
\mathrm{ENGE} \times \text { HTOF } 1 \mathrm{X} \times \mathrm{HTOF} 2 \mathrm{X} \times \alpha \\
\end{array}$ & $\begin{array}{c}\text { Beam charge } \\
{[\mathrm{mC}]} \\
\end{array}$ \\
\hline $\begin{array}{c}\# 12 \\
(57729-57830, \\
58401-58409)\end{array}$ & 21.6 & $\overline{12} \mathrm{C}$ & $\overline{\mathrm{ON}}$ & $\alpha \alpha=H T O F 1 Y \times \mathrm{W} \check{C}(\mathrm{~T} \otimes \mathrm{B}) \times \overline{\mathrm{AC}}(\mathrm{T} \otimes \mathrm{B})$ & 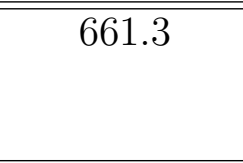 \\
\hline $\begin{array}{c}\# 13 \\
(57258-57265, \\
57837-58394)\end{array}$ & 12.5 & ${ }^{28} \mathrm{Si}$ & $\mathrm{ON}$ & 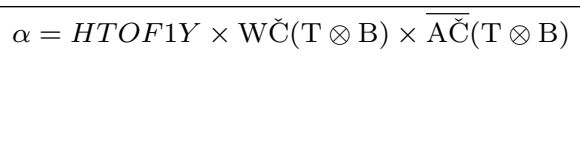 & 2776.6 \\
\hline $\begin{array}{c}\# 14 \\
(58410-58716, \\
59112-59149, \\
59479-59511, \\
59870-59991, \\
60551-60561) \\
\end{array}$ & 22.4 & ${ }^{12} \mathrm{C}$ & $\mathrm{ON}$ & 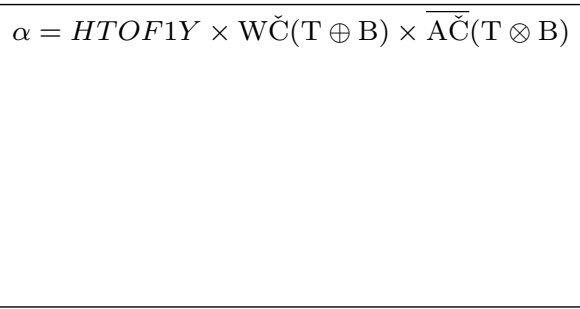 & 4633.1 \\
\hline $\begin{array}{c}\# \# 15 \\
(58719-59109, \\
59154-59315, \\
59362-59478, \\
59513-59750, \\
59801-59868, \\
59992-60019, \\
60095-60168, \\
60346-60394, \\
60562-60622, \\
60724-60756)\end{array}$ & 12.7 & ${ }^{28} \mathrm{Si}$ & $\mathrm{ON}$ & $\alpha=H T O F 1 Y \times \mathrm{W} \check{\mathrm{C}}(\mathrm{T} \oplus \mathrm{B}) \times \overline{\mathrm{AC}}(\mathrm{T} \otimes \mathrm{B})$ & 10555.4 \\
\hline $\begin{array}{c}\# 16 \\
(59316-59360, \\
59751-59800)\end{array}$ & 18.2 & ${ }^{9} \mathrm{Be}$ & $\mathrm{ON}$ & 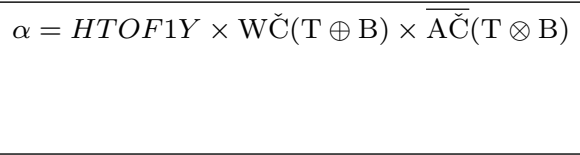 & 1316.3 \\
\hline $\begin{array}{c}\# 17 \\
(60026-60062, \\
60169-60198, \\
60395-60418)\end{array}$ & 12.4 & ${ }^{51} \mathrm{~V}$ & $\mathrm{ON}$ & $\alpha=H T O F 1 Y \times \mathrm{W} \check{\mathrm{C}}(\mathrm{T} \oplus \mathrm{B}) \times \overline{\mathrm{AC}}(\mathrm{T} \otimes \mathrm{B})$ & 699.6 \\
\hline $\begin{array}{c}\# \text { \# } 18 \\
(60063-60093, \\
60199-60232, \\
60421-60440)\end{array}$ & 10.6 & ${ }^{89} \mathrm{Y}$ & $\mathrm{ON}$ & $\alpha=H T O F 1 Y \times \mathrm{W} \check{C}(\mathrm{~T} \oplus \mathrm{B}) \times \overline{\mathrm{AC}}(\mathrm{T} \otimes \mathrm{B})$ & 516.0 \\
\hline $\begin{array}{c}\# 19 \\
(60236-60354, \\
60441-60549 \\
61036-61068)\end{array}$ & 24.9 & ${ }^{7} \mathrm{Li}$ & $\mathrm{ON}$ & 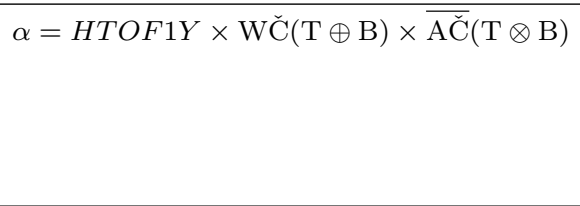 & 3467.6 \\
\hline $\begin{array}{c}\text { \# } 20 \\
(60623-60723, \\
60757-60809, \\
60963-60981, \\
61015-61035)\end{array}$ & 20.5 & ${ }^{6} \mathrm{Li}$ & $\mathrm{ON}$ & 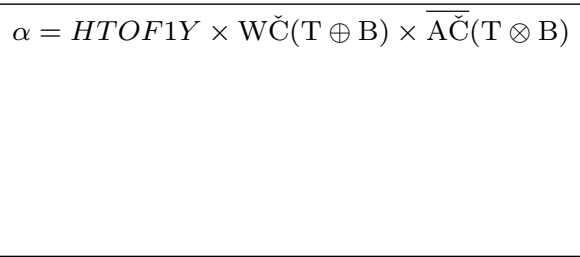 & 2273.3 \\
\hline $\begin{array}{c}\# 21 \\
(60812-60961)\end{array}$ & 26.5 & ${ }^{10} \mathrm{~B}$ & $\mathrm{ON}$ & 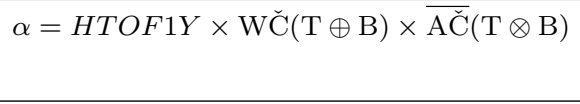 & 3099.1 \\
\hline $\begin{array}{c}\# 22 \\
(60982-61013)\end{array}$ & 0.9 & ${ }^{208} \mathrm{~Pb}$ & $\mathrm{ON}$ & 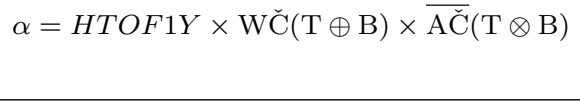 & 16.5 \\
\hline
\end{tabular}


Table 2.14: Run summary.

\begin{tabular}{c|c|c|c|c}
\hline Target & $\begin{array}{c}\text { Thickness } \\
{\left[\mathrm{mg} / \mathrm{cm}^{2}\right]}\end{array}$ & $\begin{array}{c}\text { Total beam charge } \\
{[\mathrm{mC}]}\end{array}$ & $\begin{array}{c}\text { Run time } \\
{[\text { hours }]}\end{array}$ & Notes \\
\hline \hline $\mathrm{CH}_{2}$ & 460 & 679.0 & 82.2 & for mass calibration \\
${ }^{6} \mathrm{Li}$ & 164 & 2273.3 & 34.3 & \\
${ }^{7} \mathrm{Li}$ & 189 & 3467.6 & 42.3 & \\
${ }^{9} \mathrm{Be}$ & 188 & 3683.8 & 56.9 & \\
${ }^{10} \mathrm{~B}$ & 114 & 3099.1 & 35.1 & \\
${ }^{12} \mathrm{C}$ & 100 & 13979.7 & 190.6 & \\
${ }^{28} \mathrm{Si}$ & 65 & 13332.0 & 331.9 & \\
${ }^{51} \mathrm{~V}$ & 59.6 & 699.6 & 16.9 & \\
${ }^{89} \mathrm{Y}$ & 56 & 516.0 & 15.3 & \\
${ }^{208} \mathrm{~Pb}$ & 283 & 16.5 & 5.7 & for QF rate study \\
\hline
\end{tabular}




\section{Chapter 3}

\section{Performance of the water Čerenkov counter}

\subsection{Overview}

$\Lambda$ hypernuclear spectroscopy has been extensively investigated by a wide variety of reactions such as $\left(\mathrm{K}^{-}, \pi^{-}\right)$and $\left(\pi^{+}, \mathrm{K}^{+}\right)$at CERN-PS [15, 19, 22], BNL-AGS [23-25] and KEK $12 \mathrm{GeV}$ PS $[28,31]$ since the 1970s. To date, hypernuclear mass spectra up to mass number 208 have been measured with an energy resolution of $1.45-2.0 \mathrm{MeV}$ (FWHM). In addition to the mesonic reactions, $\left(\mathrm{e}, \mathrm{e}^{\prime} \mathrm{K}^{+}\right)$reactions were also introduced at JLab in recent years. A pioneering experiment, JLab E89-009, was successfully carried out with sub MeV resolution in 2000 [46].

From an experimental point of view, kaon identification is very important to cleanly measure $\Lambda$ hypernuclear mass spectra in any of these reactions. For the mesonic reaction experiment, $p=0.5 \sim 0.7 \mathrm{GeV} / c$ kaons were identified with an acrylic sheet as a Cerenkov radiator. On the other hand, for the $\left(\mathrm{e}, \mathrm{e}^{\prime} \mathrm{K}^{+}\right)$reaction experiment in Hall $\mathrm{C}$ at JLab in which a kaon was measured in the momentum region of $1.2 \mathrm{GeV} / c$, the acrylic radiator does not have sufficient $\mathrm{p} / \mathrm{K}^{+}$separation power due to the large refractive index of 1.49 .

For the next $\Lambda$ hypernuclear spectroscopic experiment through the (e, $\left.\mathrm{e}^{\prime} \mathrm{K}^{+}\right)$reaction in the JLab E01-011 experiment [71], particle identification in a hadronic arm is even more essential since proton and pion rates are expected to be much higher. In order to overcome the situation, we designed and developed an array of water Čerenkov counters that reject protons at the kaon trigger level by $\sim 10^{-4}$.

During the R\&D stage, we investigated optical properties of reflector materials and a wave length shifter used for the water Čerenkov counter.

Performance of prototype counters were tested using unseparated beams at the test beam line of KEK $12 \mathrm{GeV}$ PS in the momentum region of 1.05 to $1.35 \mathrm{GeV} / c$, which is exactly the region of interest in E01-011.

Since the R\&D of the water Cerenkov counter is one of the major contributions by the author, detailed discussion about the performance of the water Cerenkov counter is held in this chapter. We describe design considerations containing requirements, reflectors and wavelength shifter research in Section 3.2. Counter performance with unseparated beam at KEK 12 GeV PS and the real experiment are described in Section 3.3. 


\subsection{Design consideration}

\subsubsection{Requirements}

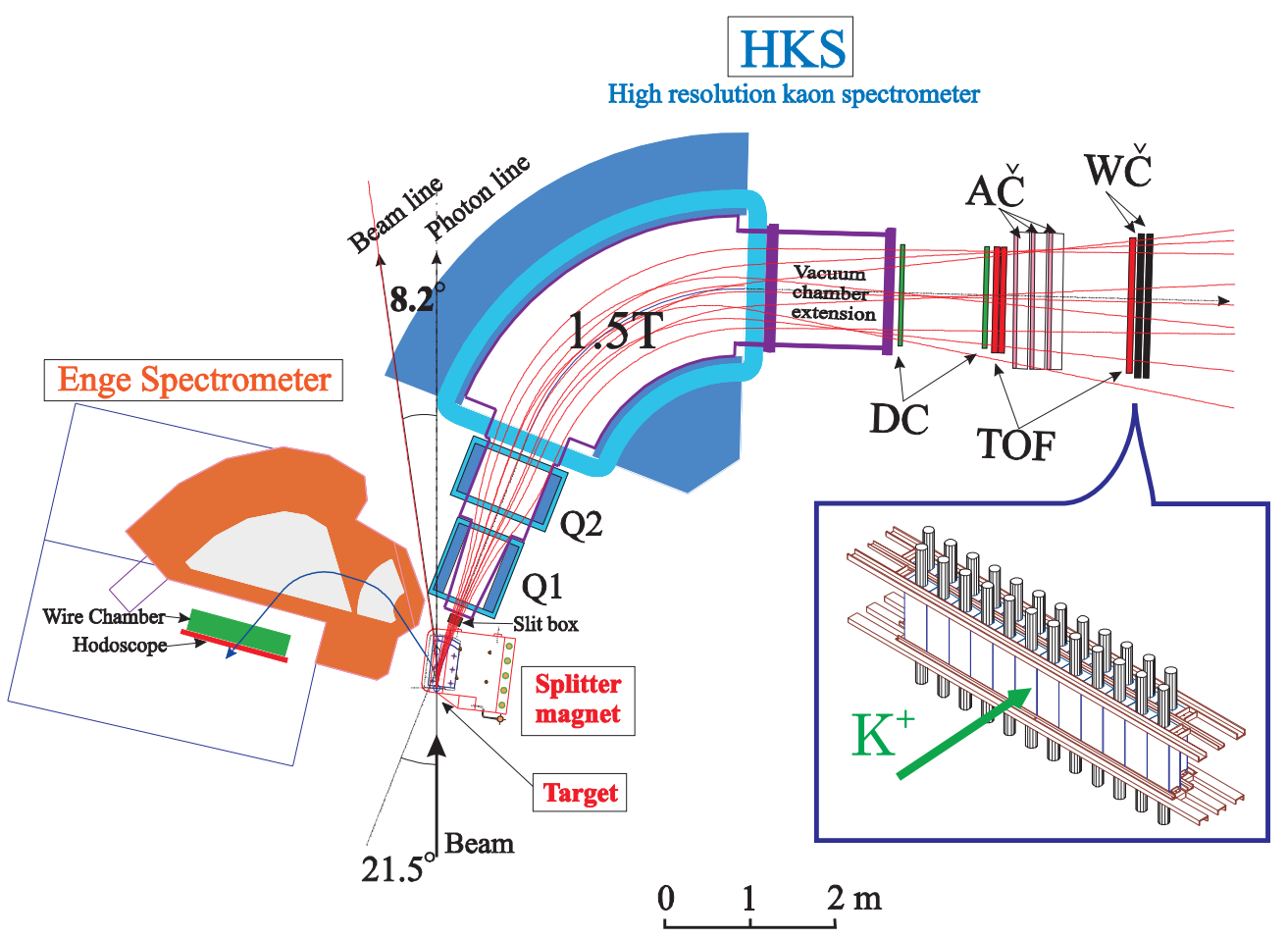

Figure 3.1: Top view of the E01-011 experimental setup.

Fig. 3.1 shows a schematic view of the E01-011 experimental setup. An incident electron beam of $\sim 1.8 \mathrm{GeV}$ hits a target, and a kaon and a scattered electron at very forward angles are deflected to the High resolution Kaon Spectrometer (HKS) and the scattered electron spectrometer, respectively, by the splitter magnet. The HKS consists of $\mathrm{Q}+\mathrm{Q}+\mathrm{D}$ magnets and momentum analyzes kaons in momentum region of $1.2 \mathrm{GeV} / c \pm 12.5 \%$. $0.3 \mathrm{GeV} / c \pm 30 \%$ electrons were measured with the scattered electron spectrometer.

The HKS detector package, as shown in Fig. 3.1, consists of two drift chambers, two layers of TOF counters, three layers of aerogel Cerenkov counters and two layers of water Čerenkov counters. The water Čerenkov counters were installed most downstream because of their large radiation length.

In the design phase of E01-011, HKS singles rates were estimated. These are given for the two typical targets, ${ }^{12} \mathrm{C}$ and ${ }^{28} \mathrm{Si}$ in Table 3.3 .

Hadronic rates are expected to be almost three order of magnitudes higher in E01-011 than in the previous experiment, E89-009. Therefore, it is important to suppress protons and pions at the kaon trigger level in order to achieve high yield rates of $\Lambda$ hypernuclei.

We prepared aerogel Čerenkov counters for $\pi^{+} / \mathrm{K}^{+}$particle identification, which is described in [63].

For $\mathrm{p} / \mathrm{K}^{+}$particle identification which requires suppression efficiency of $\sim 10^{-4}$, it is necessary to replace the acrylic Cerenkov counters used in the previous experiment by water ones.

Fig. 3.2 shows dependence of number of photons on particle momentum for two radiator materials, water $(n=1.33)$ and acryl $(n=1.49)$, respectively. 


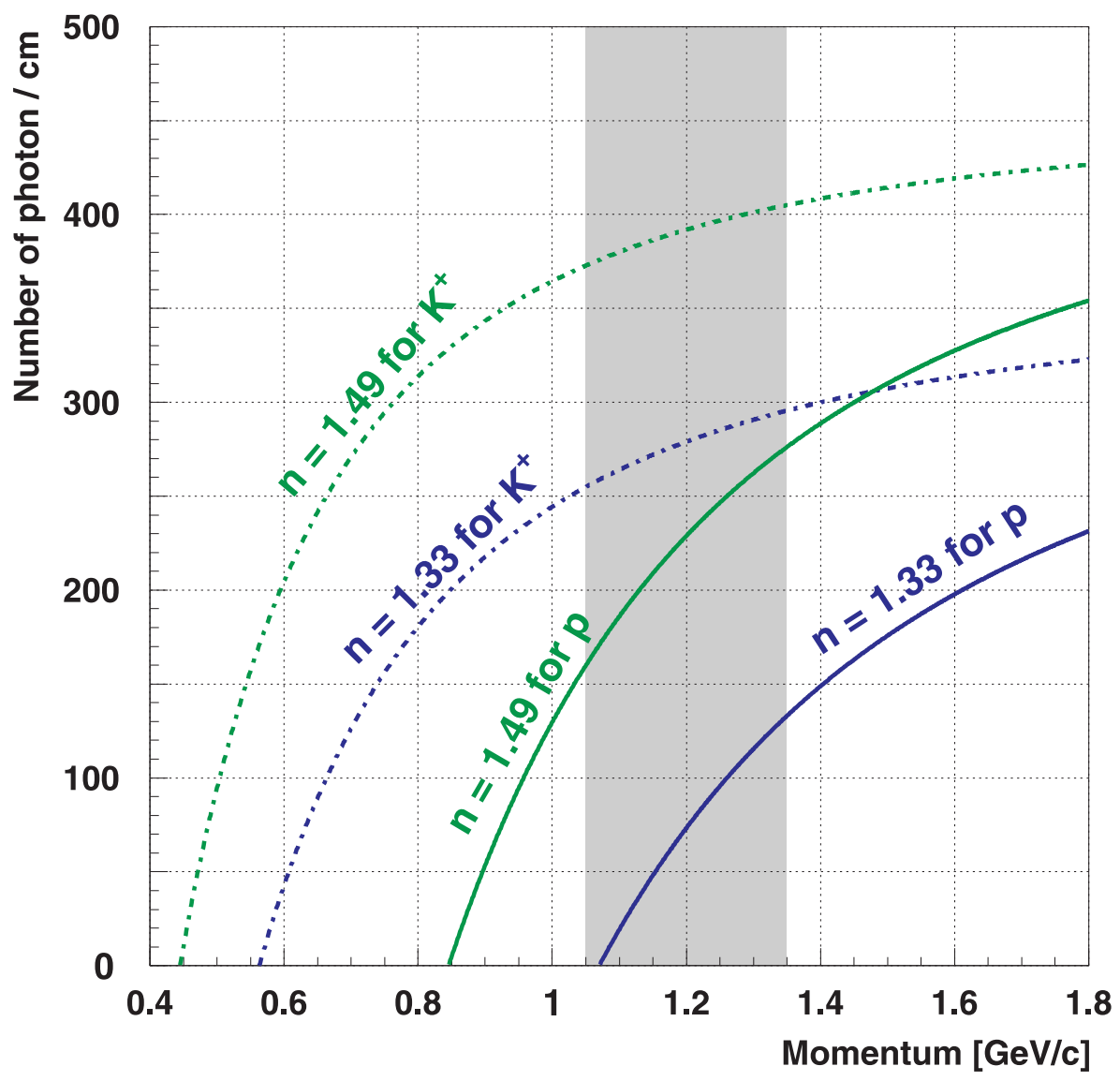

Figure 3.2: Particle momentum dependence of the number of photons. The refractive index $n=1.33$ and 1.49 indicate water and acrylic radiator, respectively. 
The momentum range of interest, $1.05 \sim 1.35 \mathrm{GeV} / c$, is designated by the hatched area, and is well above the Cerenkov threshold for protons with the acryl as opposed to the water.

For example, the ratio of the number of photons merged over the momentum range is evaluated to be $56 \%$ for the Lucite radiator, while $22 \%$ for the water radiator. Thus the water has almost twice separation power for $\mathrm{p} / \mathrm{K}^{+}$than that for the acryl.

\subsubsection{Container design}

One layer of the water Čerenkov counter consists of 12 segments.

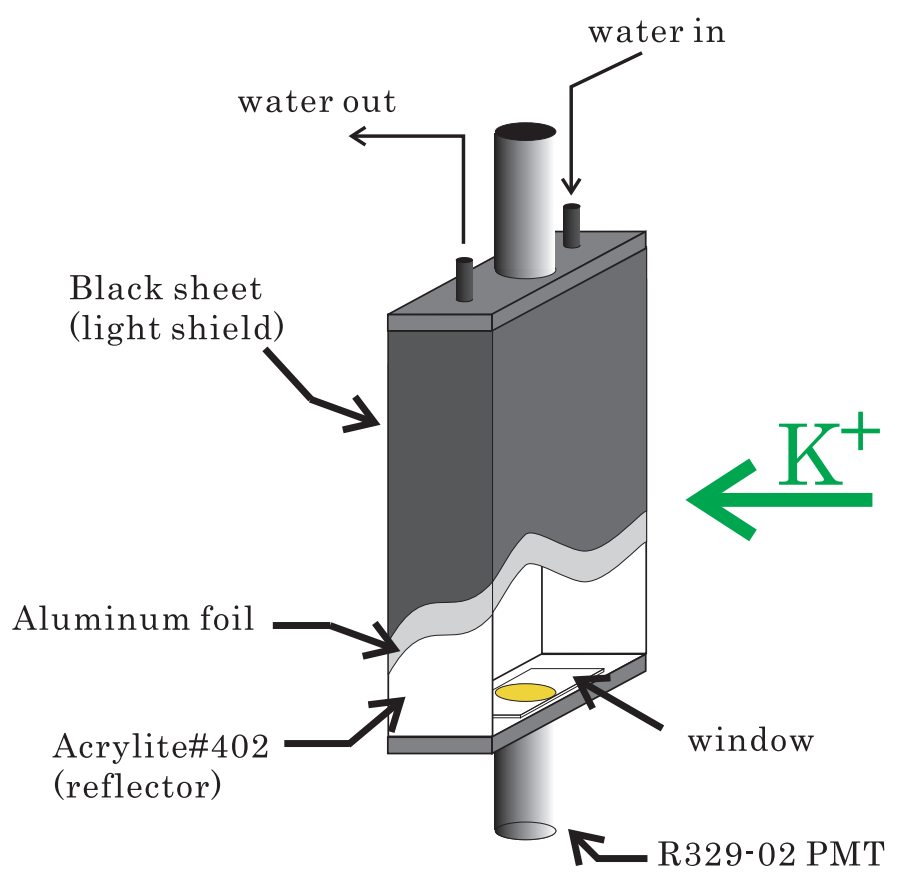

Figure 3.3: Schematic drawing of the water Čerenkov counter.

Fig. 3.3 shows a schematic structure of one segment of the water Cerenkov counter. The effective radiator volume of a segment is $350^{H} \times 150^{W} \times 75^{T} \mathrm{~mm}^{3}$. The water containers are made of white cast acrylic sheet (Mitsubishi Rayon Co.,Ltd.; Acrylite \#402) with two UVT acrylic transparent windows (Mitsubishi Rayon Co.,Ltd.; Acrylite \#000) viewed from both ends by 2" $\phi$ phototubes (Hamamatsu; R329-02). Amino G-salt (Di-potassium salt of 2-amino-6, 8naphthalene-disulphonic acid), a wave length shifter, is dissolved into de-ionized water $(\sim 18$ $\mathrm{M} \Omega \cdot \mathrm{cm})$. Details of the wave length shifter are discussed in Section 3.2.3.

Each container is wrapped with aluminum foil and black plastic sheet as a light shield. It has two plugs for water charge and discharge.

Parameters of the water Čerenkov counter are listed in Table 3.1.

\subsubsection{Reflector and wavelength shifter}

Before we chose the white cast acrylic sheet (Acrylite \#402) as a water container material, relative diffusion reflectance for various kinds of materials were measured with a spectrophotometer (Shimadzu UV-2400 and ISR-240A). A schematic drawing of the spectrophotometer is shown in Fig. 3.4 and the specification is listed in Table 3.2.

Barium sulfate $\left(\mathrm{BaSO}_{4}\right)$ powder, DIN 5033 (Merck Co.,INC.) was adopted as a white standard 
Table 3.1: The water Čerenkov counter parameters.

\begin{tabular}{lc}
\hline \hline Radiator volume & $35^{H} \times 15^{W} \times 7.5^{T} \mathrm{~cm}^{3}$ \\
& $\cong 4 l, 5 \mathrm{~kg}$ with 2 PMT \\
Container material & Acrylite \#402 (Mitsubishi) \\
Total reflection & $93 \%$ \\
Total transmittance & $7 \%$ \\
Lens material & Acrylite \#000 (Mitsubishi) \\
Phototube & R329-02 (Hamamatsu) $\times 2$ \\
\hline \hline
\end{tabular}

reference for relative diffuse reflectance measurement. The absolute reflectance of $\mathrm{BaSO}_{4}$ is shown as Fig. 3.5. In the Fig. 3.4, reference and sample lights are independently applied to a sample and the standard reference $\left(\mathrm{BaSO}_{4}\right)$, respectively. Both incident and reflection light are detected by the phototube mounted on the center bottom of the diffusion box.

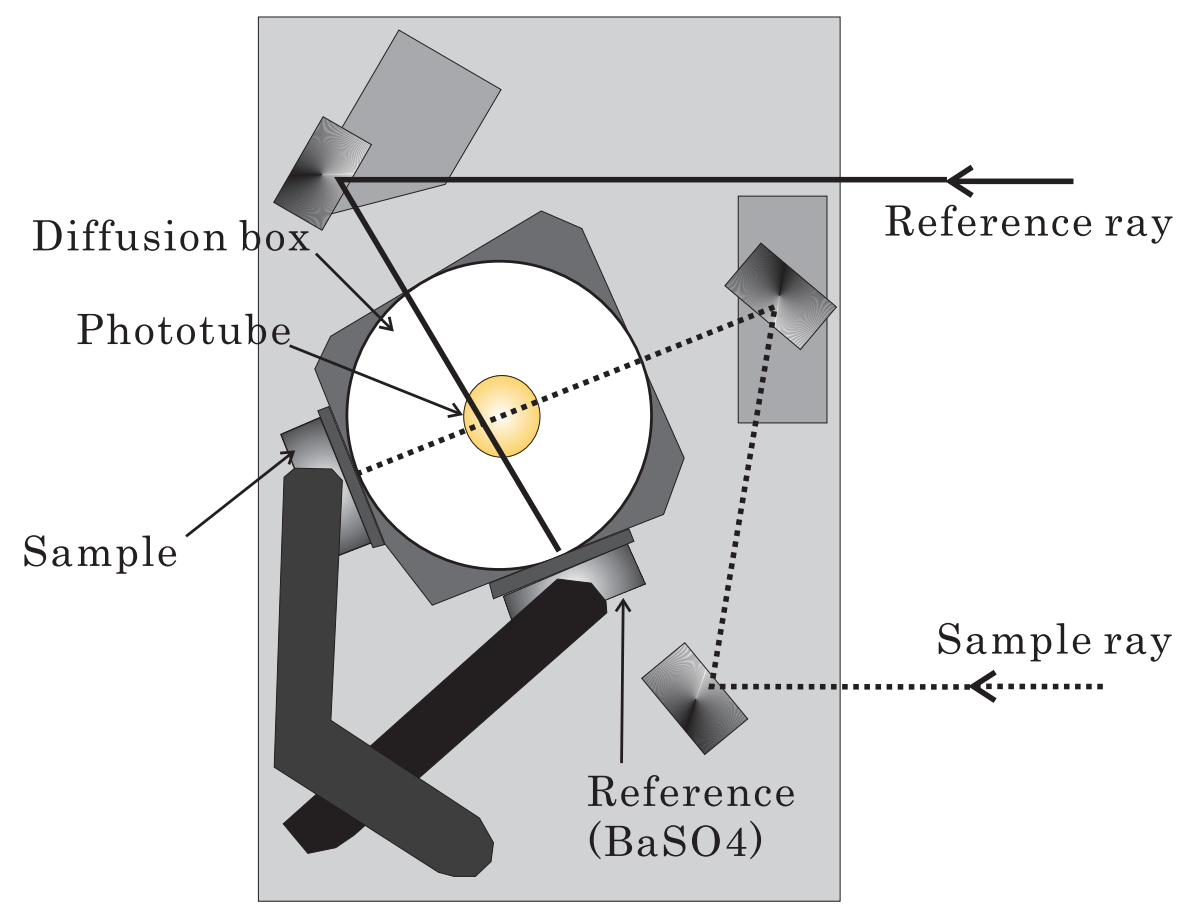

Figure 3.4: Schematic view of the spectrophotometer (Shimadzu UV-2400 and ISR-240A).

For the reflectors of the Čerenkov counter, the following four materials were tested; a hydrophilic membrane; INYU00010 (Millipore Corporation), a hydrophobic membrane; GoreTex $0.5 \mathrm{~mm}$ DPR (W.L. Gore \& Associates, Inc.), silver plated electro chemical buffed stainless steel plate (Ultra Finish Technology Co., Ltd.) and white cast acrylic sheet (Acrylite \#402). The measured relative diffuse reflectances for the four materials are presented in Fig. 3.6. For the hydrophilic and the hydrophobic membrane, there are some regions where the relative diffuse reflectances are greater than 100. This is because diffuse reflectance of each material itself is much higher than $\mathrm{BaSO}_{4}$.

The total relative diffuse reflectances of both hydrophilic and hydrophobic membranes are almost $100 \%$ around ultraviolet region and gradually decrease in the visible wave length region in the case of the hydrophobic membrane. 
Table 3.2: Specification of the spectrophotometer.

\begin{tabular}{lc}
\hline \hline Measurable wavelength range & $220 \sim 850 \mathrm{~nm}$ \\
Wavelength scanning speed & \\
Wavelength slew rate & $\sim 3200 \mathrm{~nm} / \mathrm{min}$ \\
Wavelength scan rate & $900 \sim 160 \mathrm{~nm} / \mathrm{min}$ \\
Monitor scan rate & $\sim 2500 \mathrm{~nm} / \mathrm{min}$ \\
Resolution & $0.1 \mathrm{~nm}$ \\
Phototube & $\mathrm{R}-446 \mathrm{U}$ \\
Measurable max. sample size & \\
Sample & $70^{H} \times 70^{W} \times 20^{T} \mathrm{~mm}^{3}$ \\
Reference & $70^{H} \times 70^{W} \times 12^{T} \mathrm{~mm}^{3}$ \\
\hline \hline
\end{tabular}

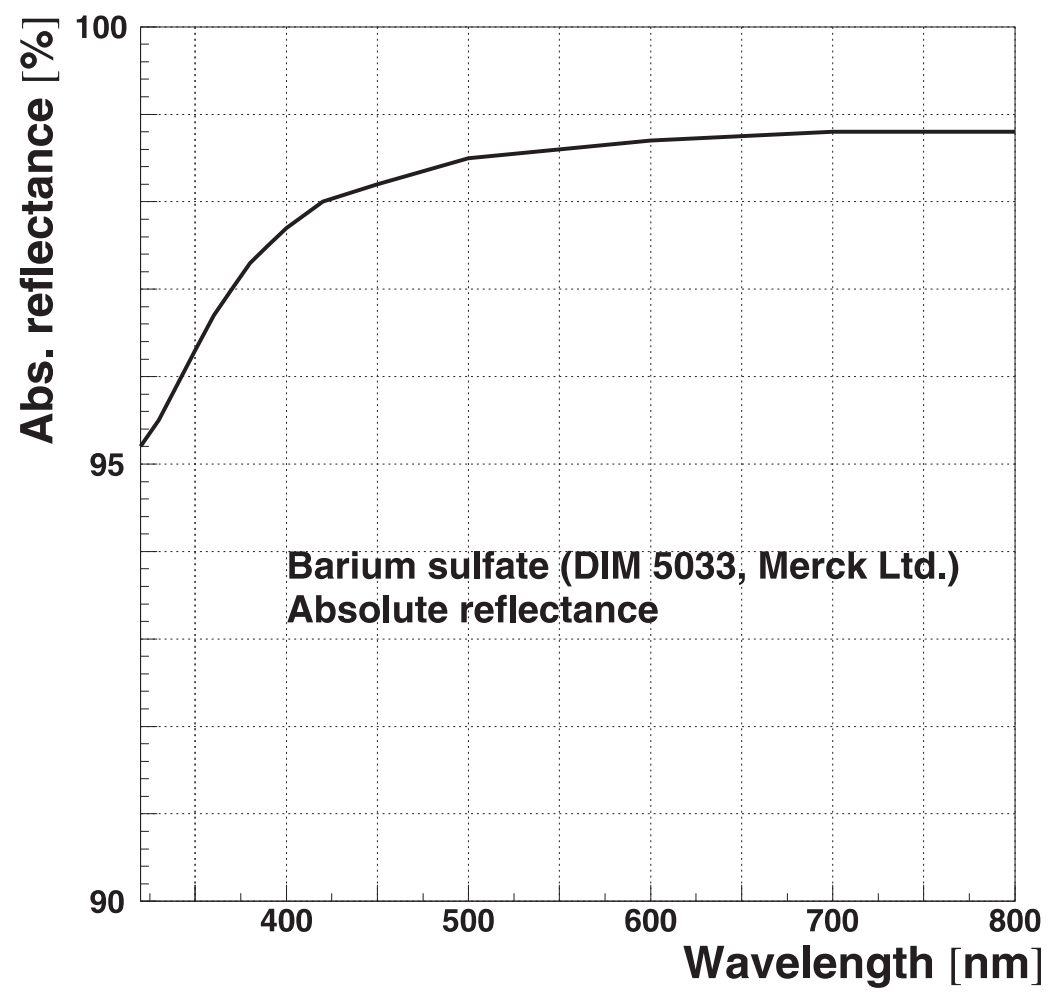

Figure 3.5: Absolute reflectance of the barium sulfate, $\mathrm{BaSO}_{4}$, the white standard for the diffusion reflectance measurements [72]. 
On the other hand, the diffuse reflectance of the white cast acrylic sheet was measured at a maximum of $94.7 \%$ around $425 \leq \lambda[\mathrm{nm}] \leq 435$, which is also the region of the phototube's maximum quantum efficiency.

In addition, the wavelength shifter solution which we selected as has a mild acidity with a $\mathrm{pH}$ of $\sim 4.5$. Finally, taking into account chemical stability with the wavelength shifter solution, we selected Acrylite \#402 cast acrylic sheet as reflector and container material.

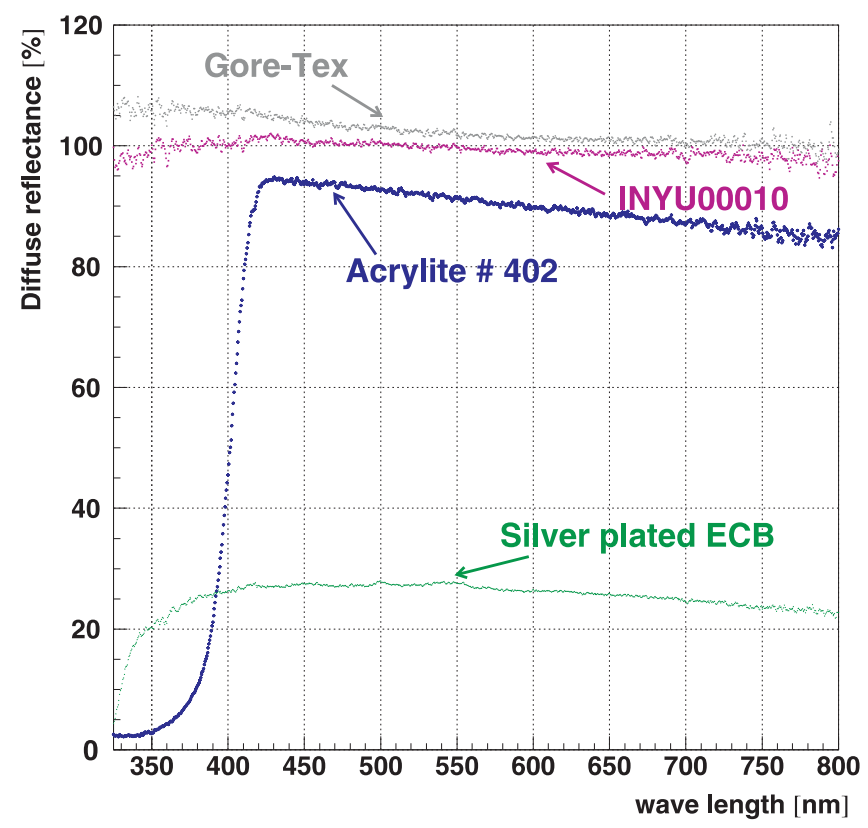

Figure 3.6: Relative diffusion reflectance for a hydrophilic membrane; INYU00010 (Millipore Corporation), a hydrophobic membrane; Gore-Tex $0.5 \mathrm{~mm}$ DPR (W.L. Gore \& Associates, Inc.), silver plated electro chemical buffed stainless steel plate (Ultra Finish Technology Co., Ltd.) and white cast acrylic sheet (Acrylite \#402).

Fluorescent solutions have been used for liquid Čerenkov counters as a function of a wavelength shifter in cosmic ray experiments since the 1950s [73].

There are several fluorescent materials such as Fluoresce [73], $\beta$-Methyl Umbelliferone [73, 74], Uvitex [73] and amino G-salt [75].

We selected the amino G-salt as a wavelength shifter since its ease to produce. Fig. 3.7 shows a density dependence of the amino G-salt $\mathrm{pH}$. The amino G-salt is mildly acidic $\mathrm{pH} \sim 4.5$ at 10 $\mathrm{mg} / l$ then gradually fall down to $\mathrm{pH} \sim 3$ as density is increased.

Since the acrylic cast sheet of reflector is a resistant alkali, and taking into account the beam test results about the number of photoelectron comparison for proton and kaon (See Section 3.3), we set the density at $10 \mathrm{mg} / l$. 


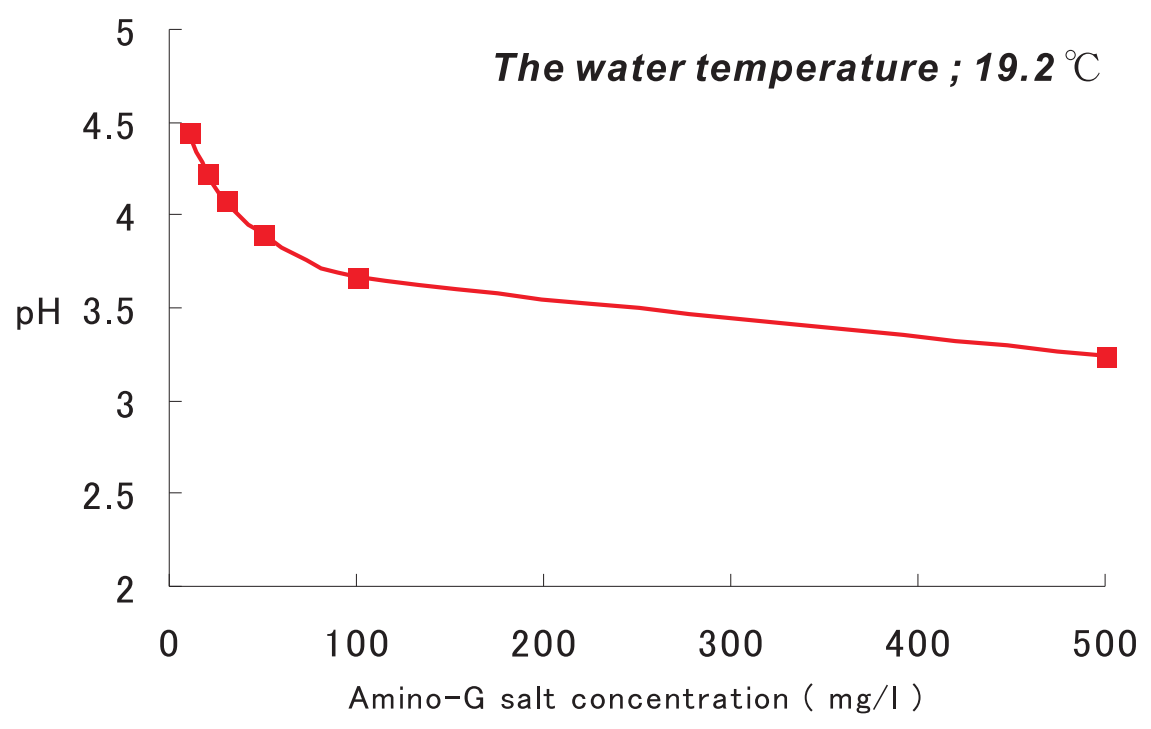

Figure 3.7: Density dependence of the amino G-salt pH.

\subsection{Performance}

\subsubsection{Beam test}

The performance of the prototype counters was investigated using unseparated beams of pions, kaons and protons provided at the KEK-PS T1 beam line, as schematically shown in Fig. 3.8. The secondary particles, which are produced in an internal target (IT : $1 \mathrm{~mm} \phi \times 20$ $\mathrm{mm}^{T}$ of tungsten) by the reaction of the $12 \mathrm{GeV}$ protons of the main ring, are transported to the $\mathrm{T} 1$ beam line with a momentum range of $0.2-2.0 \mathrm{GeV} / c$ and a momentum bite of $5 \%$. The T1 beam line was tuned to accept positively charged particles with a momentum window from 1.05 to $1.35 \mathrm{GeV} / c$, which exactly matches the requirements of the kaon spectrometer for the hypernuclear spectroscopy experiment (E01-011).

Pions, kaons and protons in the unseparated beam were identified using the information of the time of flight (TOF) counters as shown in Fig. 3.9.

Four layers of TOF counters were installed, each of which had time resolution of $77 \mathrm{ps}$ (rms). They consisted of a BICRON BC408 scintillator, acrylic light guides and Hamamatsu H1949-50 phototubes at both ends. Positrons were rejected using a gas Čerenkov counter at the trigger level. The pulse height information of the Čerenkov counters were used in the offline analysis.

A correlation of time of flight and the number of photoelectrons measured by the water Čerenkov counter, kaons being clearly identified, is shown in Fig. 3.10.

Fig. 3.11 shows distributions of the number of photoelectrons measured by the prototype water Cerenkov counters filled with the pure water with (right) and without (left) the amino G-salt wavelength shifter $(10 \mathrm{mg} / l)$.

Furthermore, the beam momentum dependence of the $\mathrm{p} / \mathrm{K}^{+}$separation was compared for the three momenta ; 1.05 (upper), 1.20 (middle) and $1.35 \mathrm{GeV} / c$ (lower). The water Čerenkov counter with the wavelength shifter gives much larger numbers of photoelectrons and clearly separates kaons from protons. 


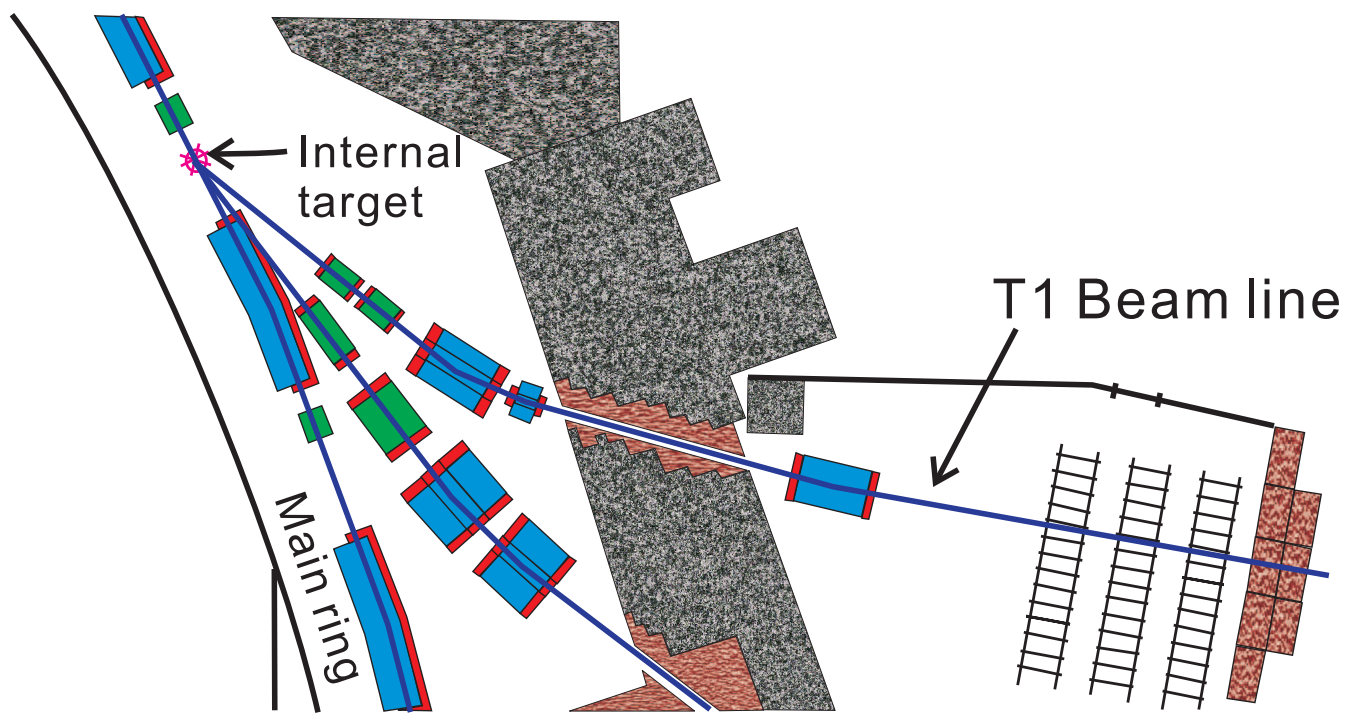

Figure 3.8: Schematic view of the KEK-PS T1 beam line.

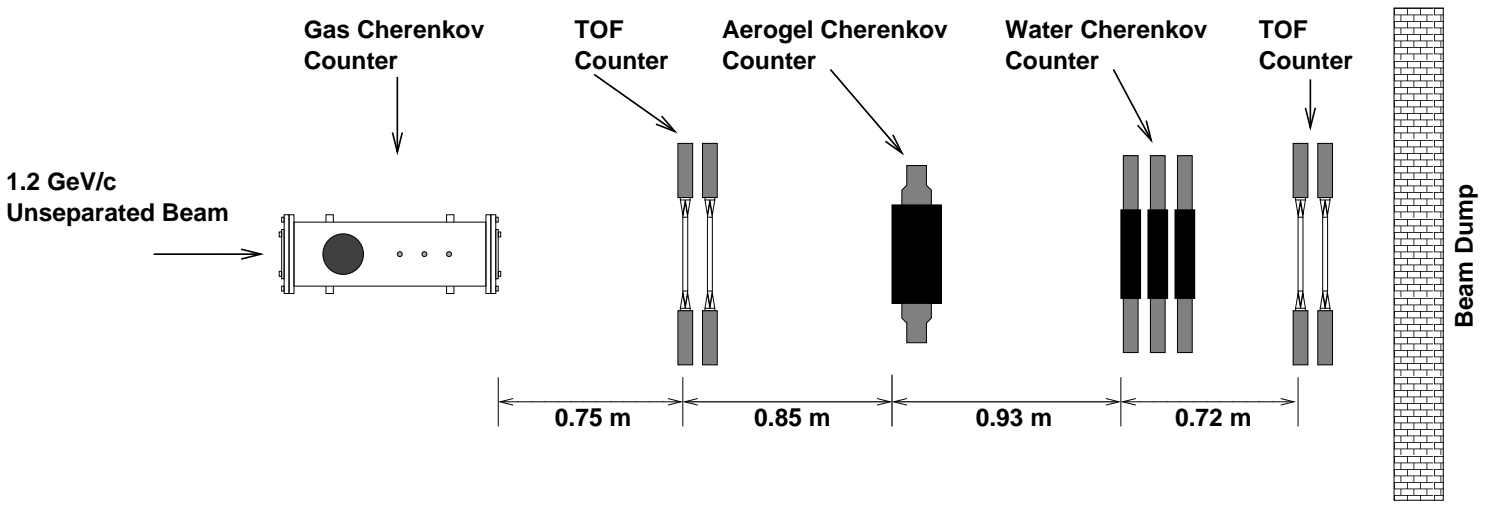

Figure 3.9: Schematic top view of the counter test performed at KEK. 


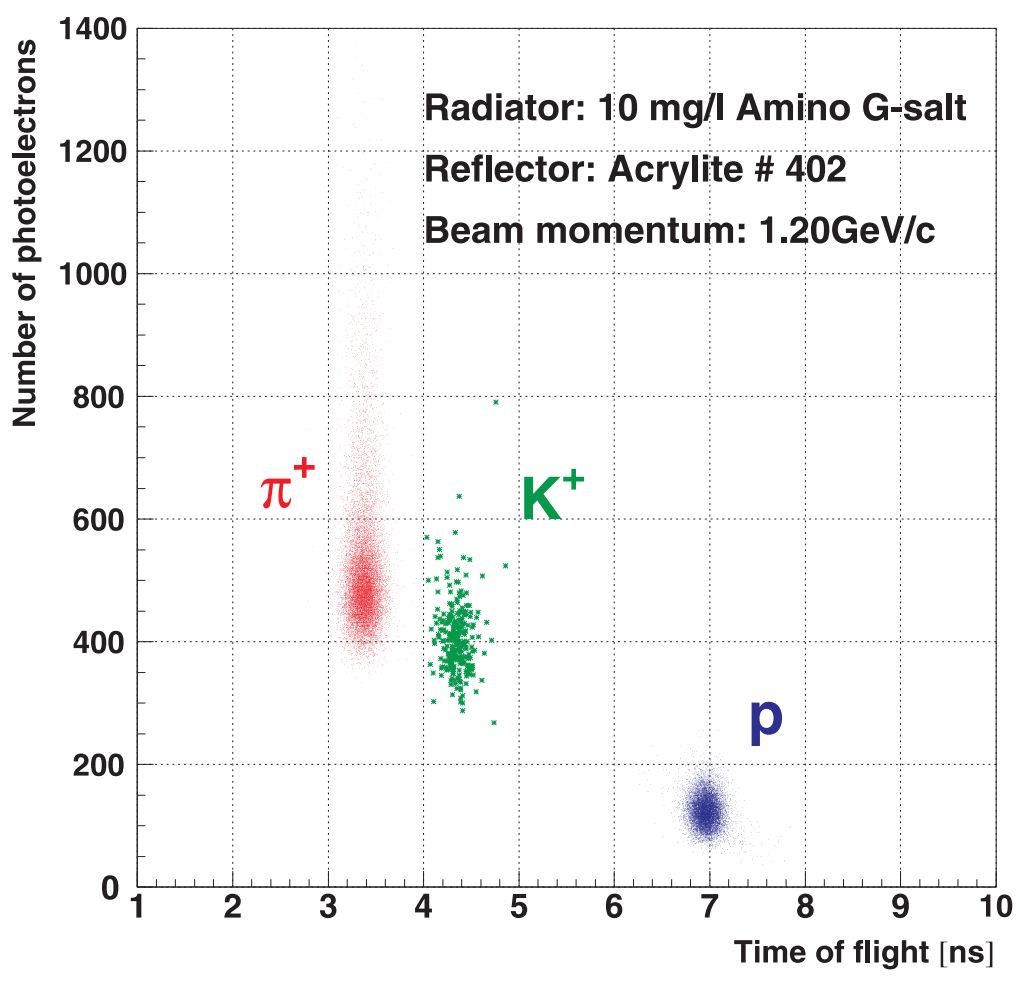

Figure 3.10: Time of flight distribution of $\pi^{+}, \mathrm{K}^{+}$and proton with particle momentum $1.2 \mathrm{GeV} / c$.

The numbers of photoelectrons for kaons $\left(N_{\text {kaon }}\right)$ and protons $\left(N_{\text {proton }}\right)$ were compared by changing the density of the amino G-salt from 1 to $2000 \mathrm{mg} / l$ as shown in Fig. 3.12.

It was found that the ratio of the number of photoelectrons for kaons and protons, $N_{\text {kaon }} / N_{\text {proton }}$, was greater than 2.5 in the density range of $1 \sim 10 \mathrm{mg} / l$, then gradually decreased with the higher density. It is also desirable to keep the solution chemically inactive so that the container is not damaged. Taking into account the $\mathrm{pH}$ measurement shown in Fig. 3.7, we set the density of the amino G-salt to $10 \mathrm{mg} / l$.

\subsubsection{Performance in the Jlab E01-011 experiment}

As described in Sec. 3.2, two layers of water Čerenkov counters were installed as one of trigger counters of the HKS in the JLab E01-011 experiment.

Measured and expected HKS singles rates for pions, protons and kaons are listed in Table 3.3. The measured rates for pions and protons were one order of magnitude less than expected.

Velocity distributions for particles detected in the HKS trigger counters $\left(\beta_{\mathrm{TOF}}-\beta_{\mathrm{K}^{+}}\right)$are shown in Fig. 4.9 with and without requiring a hit in water Cerenkov counters. $\beta_{\mathrm{TOF}}$ is the particle velocity measured by the two TOF counter walls, while $\beta_{\mathrm{K}^{+}}$is obtained from the particle momentum assuming the kaon mass.

Since during beam time, $\mathrm{p} / \mathrm{K}^{+}$separation was worse comparing one measured in several test experiment in KEK. There were some low gain counters, and it was concluded that signal discrimination for summed ADC pulse from top and bottom PMTs might improve the separation rather than for individual ADC pulse. We employed an analog signal adder for entire channels of the water Cerenkov counter from run 58410.

Fig. 3.13 shows summary of number of photon (average of 12 segments for 1 layer) for layer1 (upper) and layer2 (lower) versus run number. Generally, the number of photoelectrons decreased with time. A major possible reason for this phenomenon is the chemical stability of 

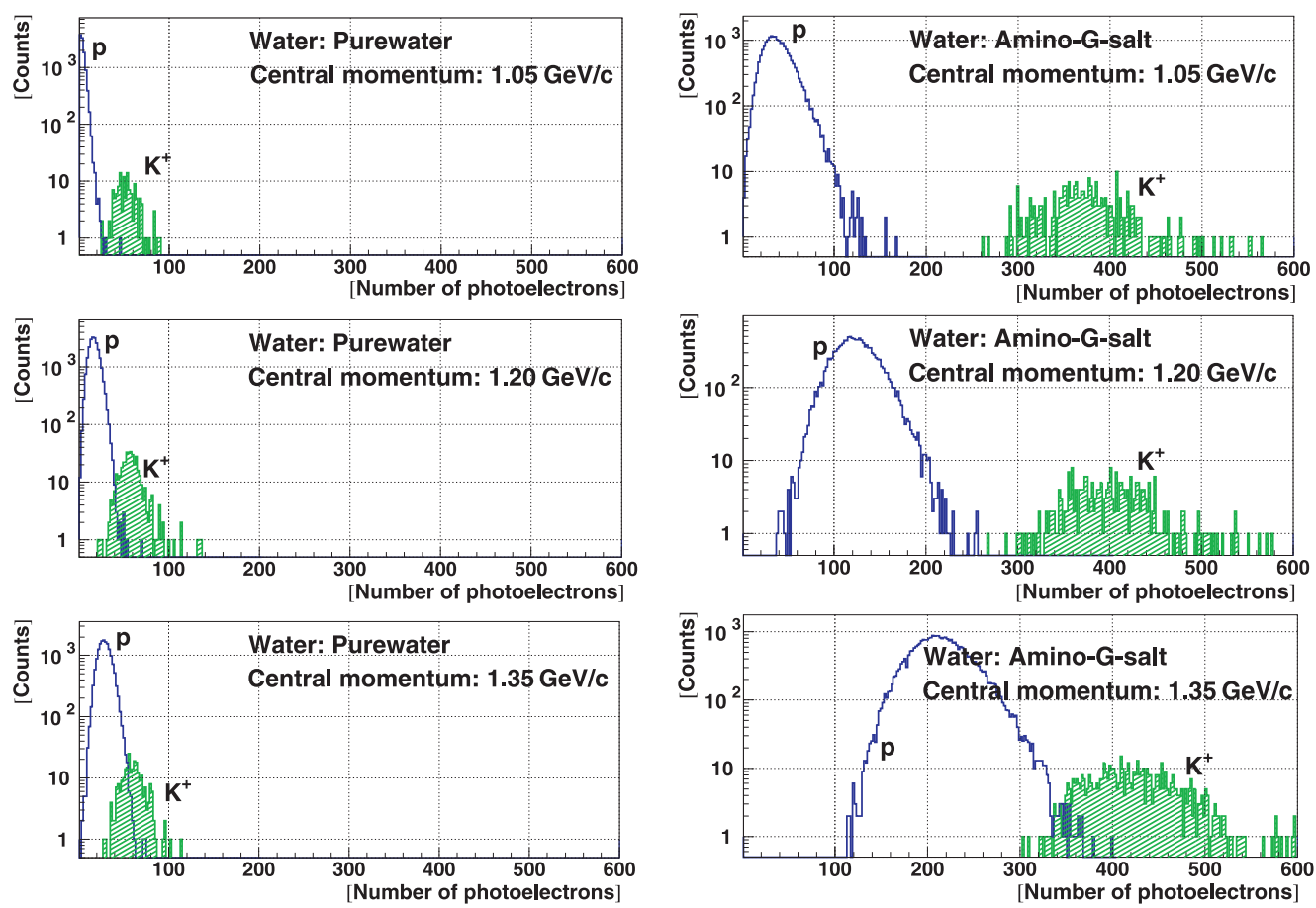

Figure 3.11: Number of photoelectron comparison with pure water (left) and chemical water (right) and with 1.05 (upper), 1.20 (middle) and $1.35 \mathrm{GeV} / c$ (lower) particle momentum sets.

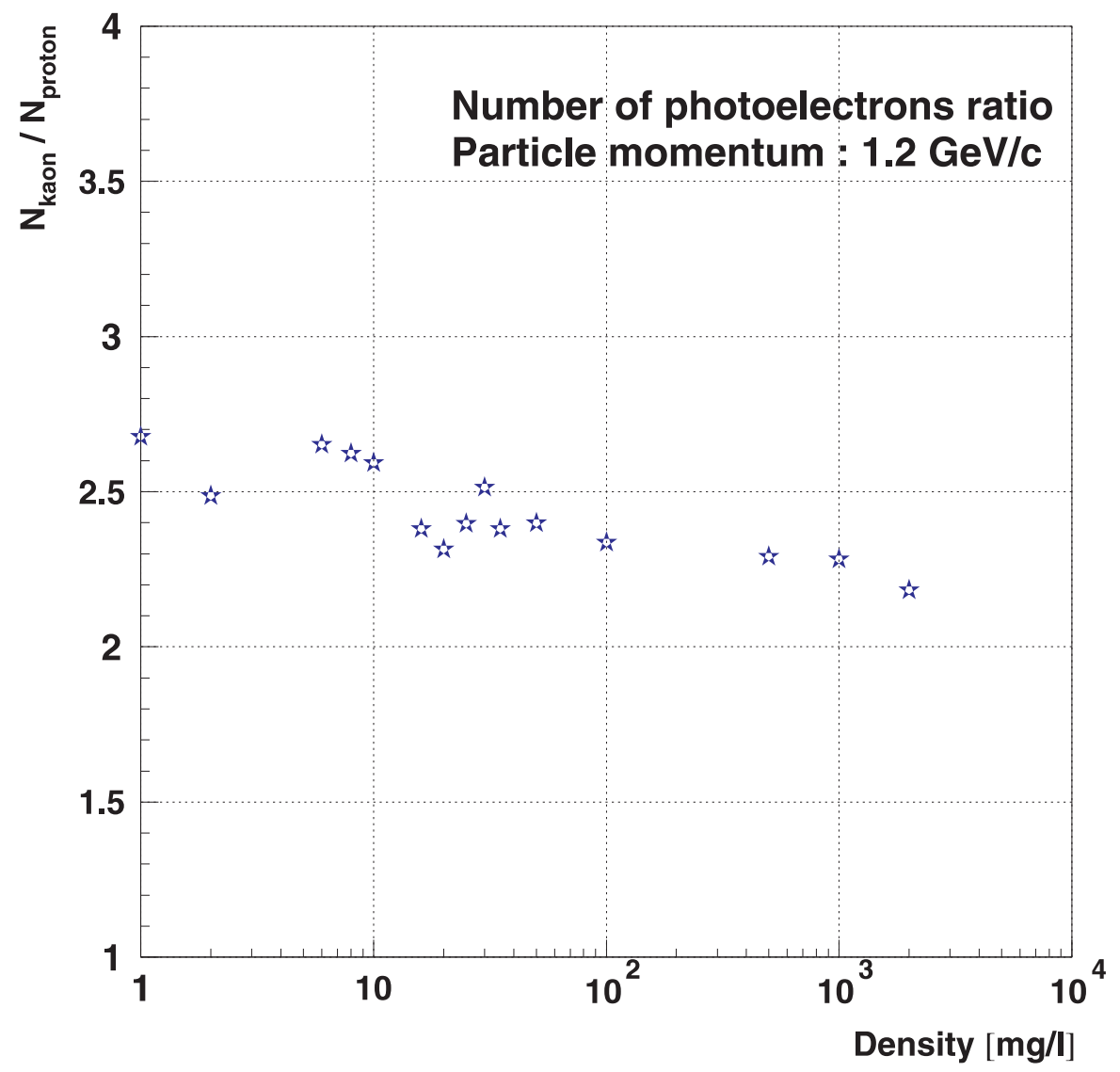

Figure 3.12: Amino G-salt density dependence on measured ratio of photoelectrons (mean) for kaons and protons. 
Table 3.3: The measured and expected HKS singles rates. Values in parentheses represent expectation.

\begin{tabular}{|c|c|c|c|c|c|}
\hline Target & $\begin{array}{l}\text { Thickness } \\
{\left[\mathrm{mg} / \mathrm{cm}^{2}\right]}\end{array}$ & $\begin{array}{l}\text { Beam intensity } \\
{[\mu \mathrm{A}]}\end{array}$ & $\begin{array}{l}\pi^{+} \\
{[\mathrm{kHz}]}\end{array}$ & $\begin{array}{l}\mathrm{K}^{+} \\
{[\mathrm{kHz}]}\end{array}$ & $\begin{array}{l}\mathrm{p} \\
{[\mathrm{kHz}]}\end{array}$ \\
\hline${ }^{12} \mathrm{C}$ & 100 & 24 & $12.8(640.0)$ & $0.2(0.27)$ & $20.0(224.0)$ \\
\hline${ }^{28} \mathrm{Si}$ & 65 & 13 & $7.5(225.3)$ & $0.1(0.08)$ & $9.0(67.6)$ \\
\hline
\end{tabular}

the salt dissolved as a wave length shifter.

Therefore, the average number of photoelectrons for each layer was normalized with the mean value subtracted by a Gaussian fit for the selected number of photoelectron distribution as kaons.

Fig. 3.14 represents the effect of the signal adder comparing normalized number of photoelectron distribution without and with the signal adder. In Fig. 3.14, hatched region corresponds to kaon and the other to proton. The signal adder suppressed protons well.

Scanning the normalized number of photoelectrons, the kaon survival rate was estimated as shown in Fig. 3.15 and defined as WC̆ efficiencies.
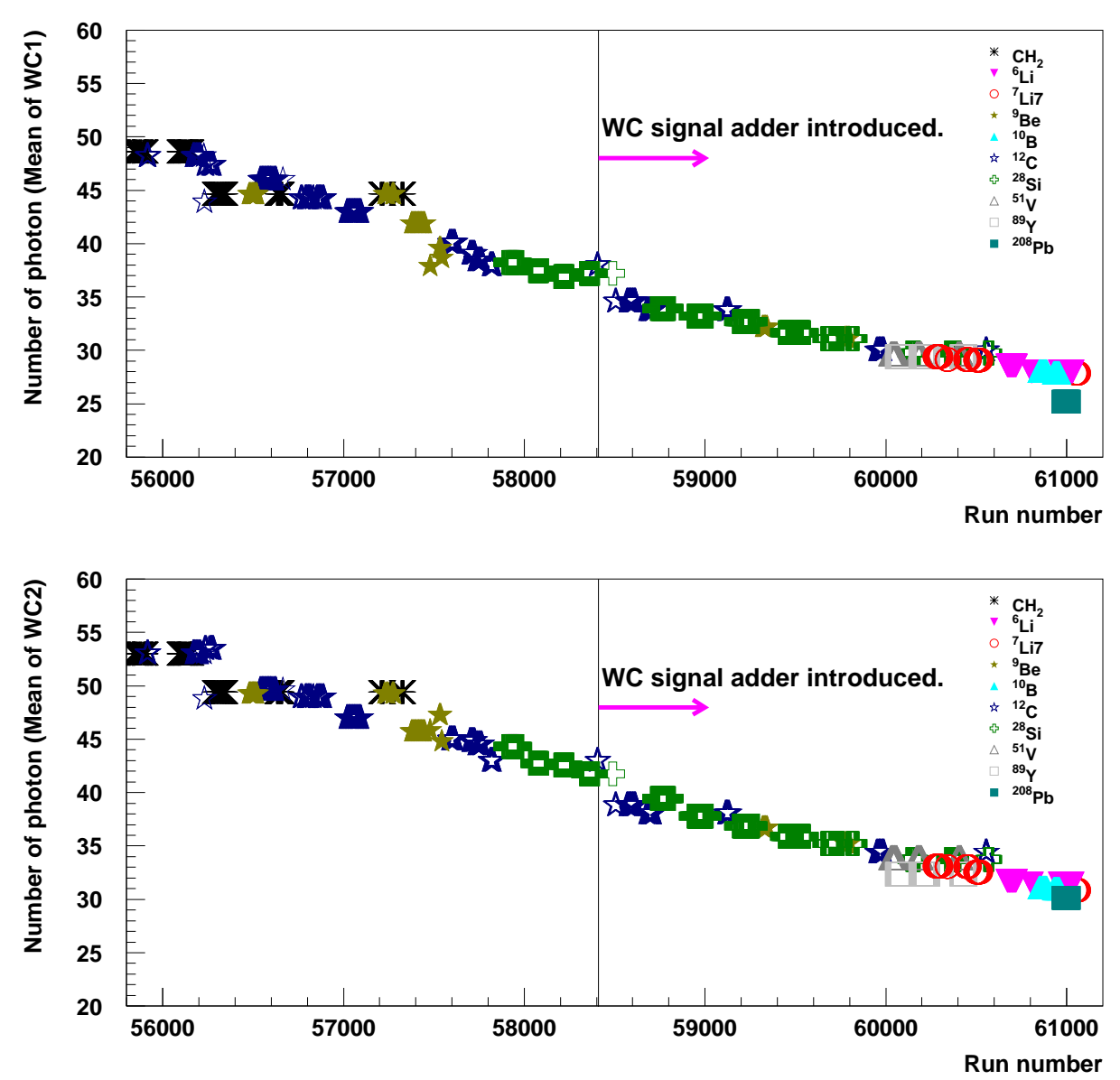

Figure 3.13: A comparison of the WČ1 (upper) and WČ2 (lower) number of photoelectrons during the entire experiment term. The number of photoelectrons were estimated as the mean of the Gaussian fit for the selected kaon events. 

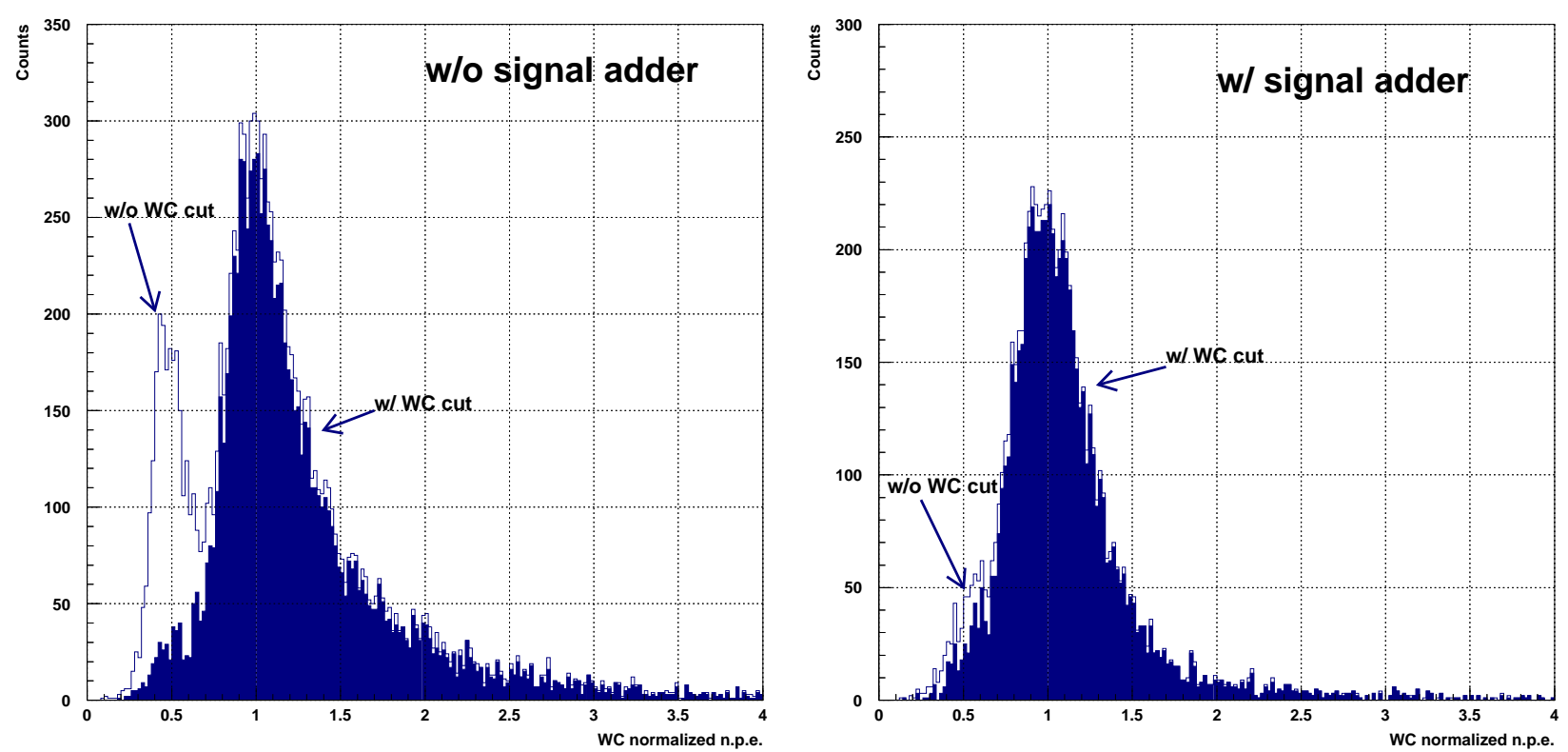

Figure 3.14: Typical WC normalized number of photoelectrons on one layer average without (left)/with (right) the signal adder.
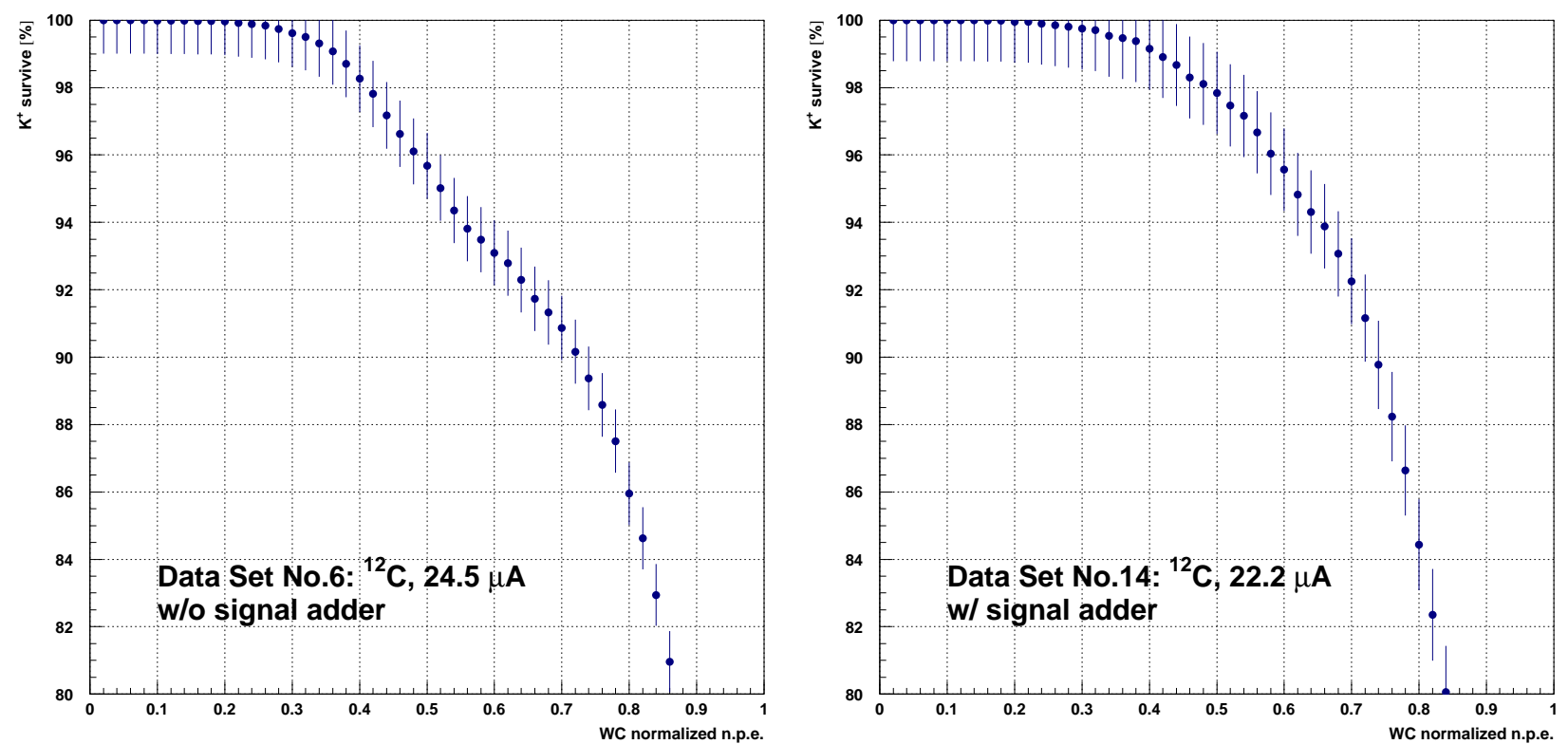

Figure 3.15: Typical WČ cut efficiencies for one layer without (left)/with (right) the signal adder. 



\section{Chapter 4}

\section{Data analysis}

\subsection{Analysis overview}

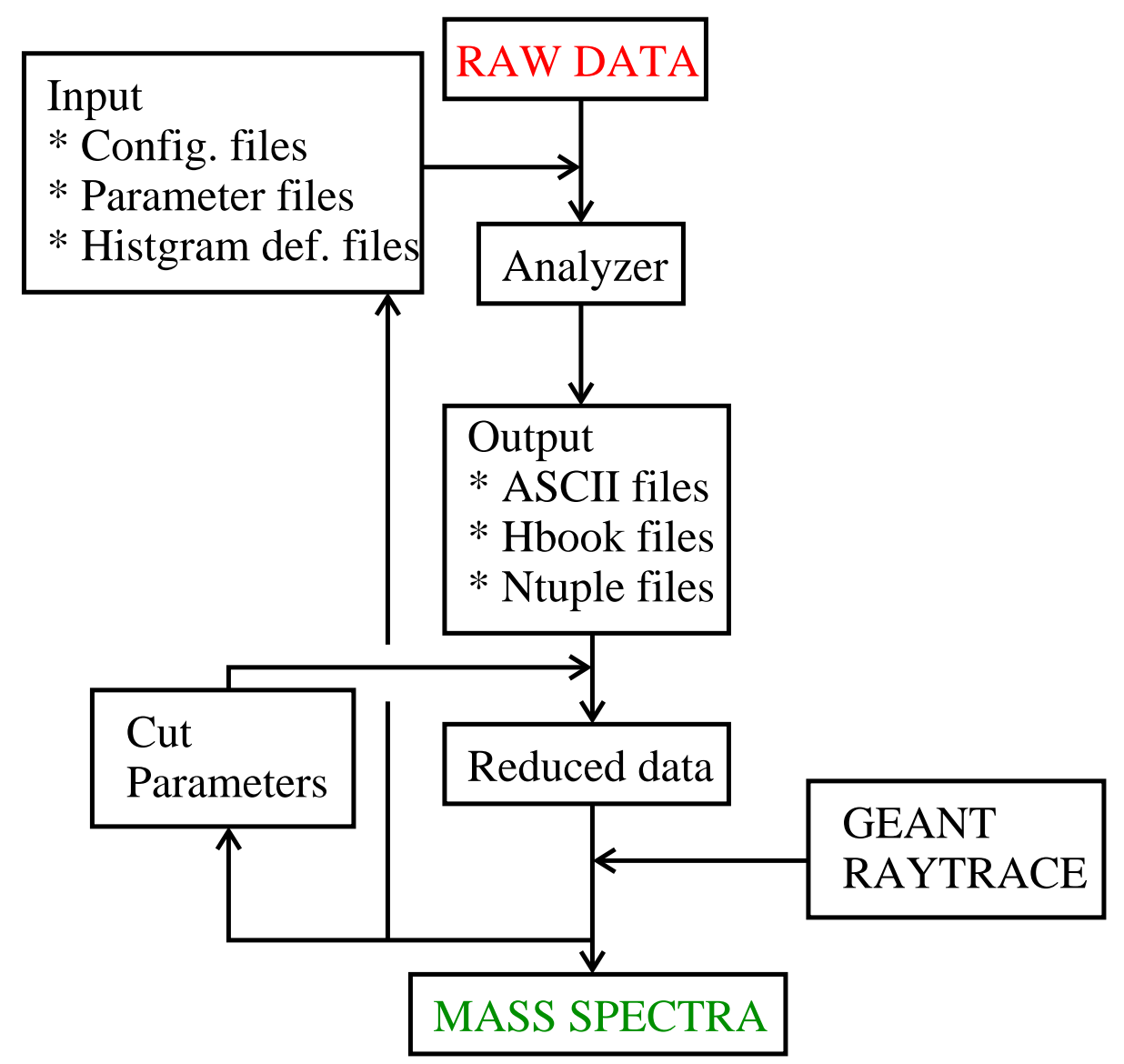

Figure 4.1: E01-011 analysis flow chart.

A flow chart of the data analysis scheme used for E01-011 is shown in as Fig. 4.1.

First, the raw data is processed by an analyzer with input definition files which describes individual detector coordinates and specifications, histogram definitions to be created, and so on. The raw data were processed by the replay software package, ENGINE [76]. Event selections were applied to the raw data in order to reduce data size and for quick analysis. The results were written to ASCII text files, Hbook histogram files and Ntuple files. Event 
selections are applied to the data to eliminate events other than kaons in the HKS within the missing mass range of interest. Real coincidence events between the HKS and the Enge were selected, and accidental coincidence events ("accidentals" which are associated with background events) are also used for evaluation of the accidentals in the real coincidence time window. The angular acceptances for the HKS and the Enge were estimated by the GEANT/RAYTRACE simulation.

Finally mass spectra and cross sections were extracted.

\subsection{Kaon measurement in HKS}

\subsubsection{Particle identification (PID)}

In order to obtain true kaon events, background particles such as positrons, pions and protons were excluded by the TOF information and the number of photoelectrons information in the aerogel and water Čerenkov counters. Methods to exclude the background particles are explained here.

\subsubsection{HKS tracking}

Particle trajectories inside the HKS detector package were determined by two drift chambers (HDC). These trajectories were used to reconstruct the angle and momentum of each event at the target.

The position and direction of particles at the focal plane : $\left(x_{f}, x_{f}^{\prime}, y_{f}, y_{f}^{\prime}\right)$, are measured. In each chamber, a series of space points are chosen based on the wire hits in each of 6 HDC planes. More than 5 plane hits are required for a space point to be determined. Then, left/right ambiguities are resolved using offsets in parallel sets of the planes such as $\mathrm{x}, \mathrm{x}$ '. The space points in chambers are then combined to make a track. The track that has the lowest $\chi^{2}$ is chosen as the true track. With this procedure, $\sim 310 \mu \mathrm{m}$ (sigma) per plane of the averaged position resolution is obtained from Fig. 4.2. The distribution of tracking $\chi^{2}$ per degree-of-freedom (d.o.f.) is also shown in Fig. 4.2.

The measured quantities at the HKS focal plane $\left(x_{f}, x_{f}^{\prime}, y_{f}, y_{f}^{\prime}\right)$ are then traced back to emission angles and momentum dispersion at the target point: $\left(x_{t}^{\prime}, y_{t}^{\prime}, d p\right)$ with transfer matrices described as the 6th order of polynomial functions of $\left(x_{f}, x_{f}^{\prime}, y_{f}, y_{f}^{\prime}\right)$. A detailed description of this reconstruction procedure is explained in Section 4.5.

\section{TOF measurement}

In the HKS detector package, the particle velocity, $\beta=v / c$, was measured by 2 layers of hodoscopes, HTOF1X and HTOF2X.

The timing of each hit of the scintillators is corrected by its pulse height, propagation time from hit position to PMT window, and any offsets arising due to differences of propagation time in electronics and cables. Accidental hits are removed by comparing tracking results to the counter hit position.

Finally, $\beta$ is obtained by fitting the corrected time at the corresponding $z$ position and using the track length of a particle. A typical $\beta$ resolution is $0.025(\sigma)$, for kaons as shown in Fig. 4.3. The particle velocity measured between HTOF1X and HTOF2X is defined as $\beta_{\text {TOF }}$ and each particle velocity $\beta_{x}\left(x=\mathrm{e}^{+}, \pi^{+}, \mathrm{K}^{+}, \mathrm{p}\right)$ is obtained assuming their mass $\left(m_{x}\right)$ and momentum 


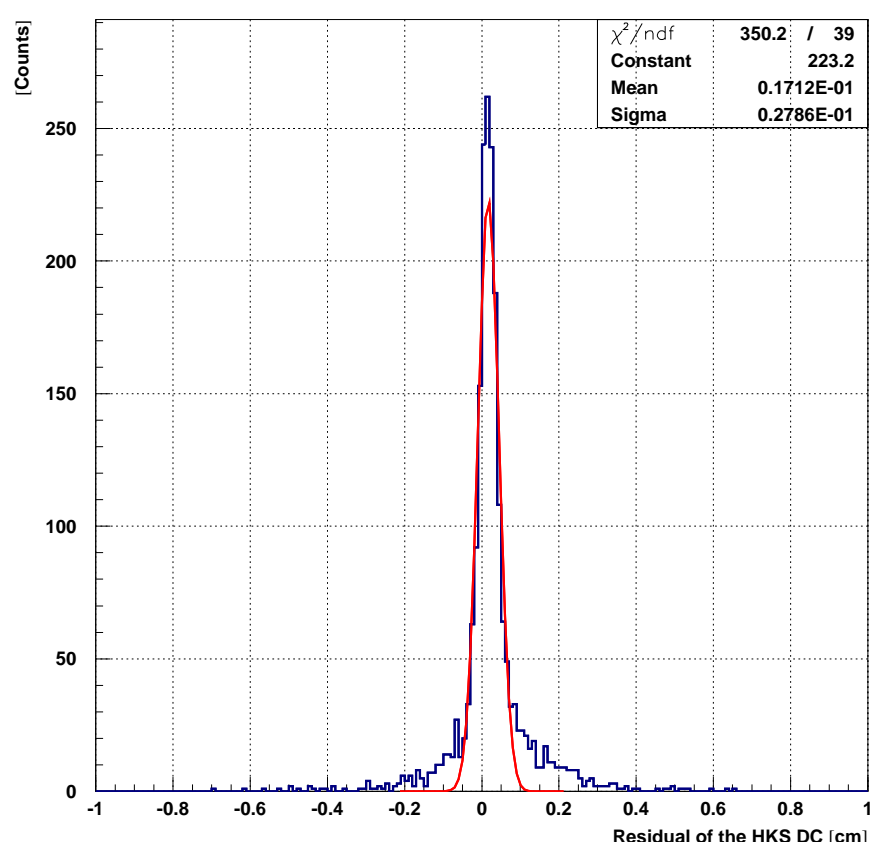

(a) Residual of the HDC

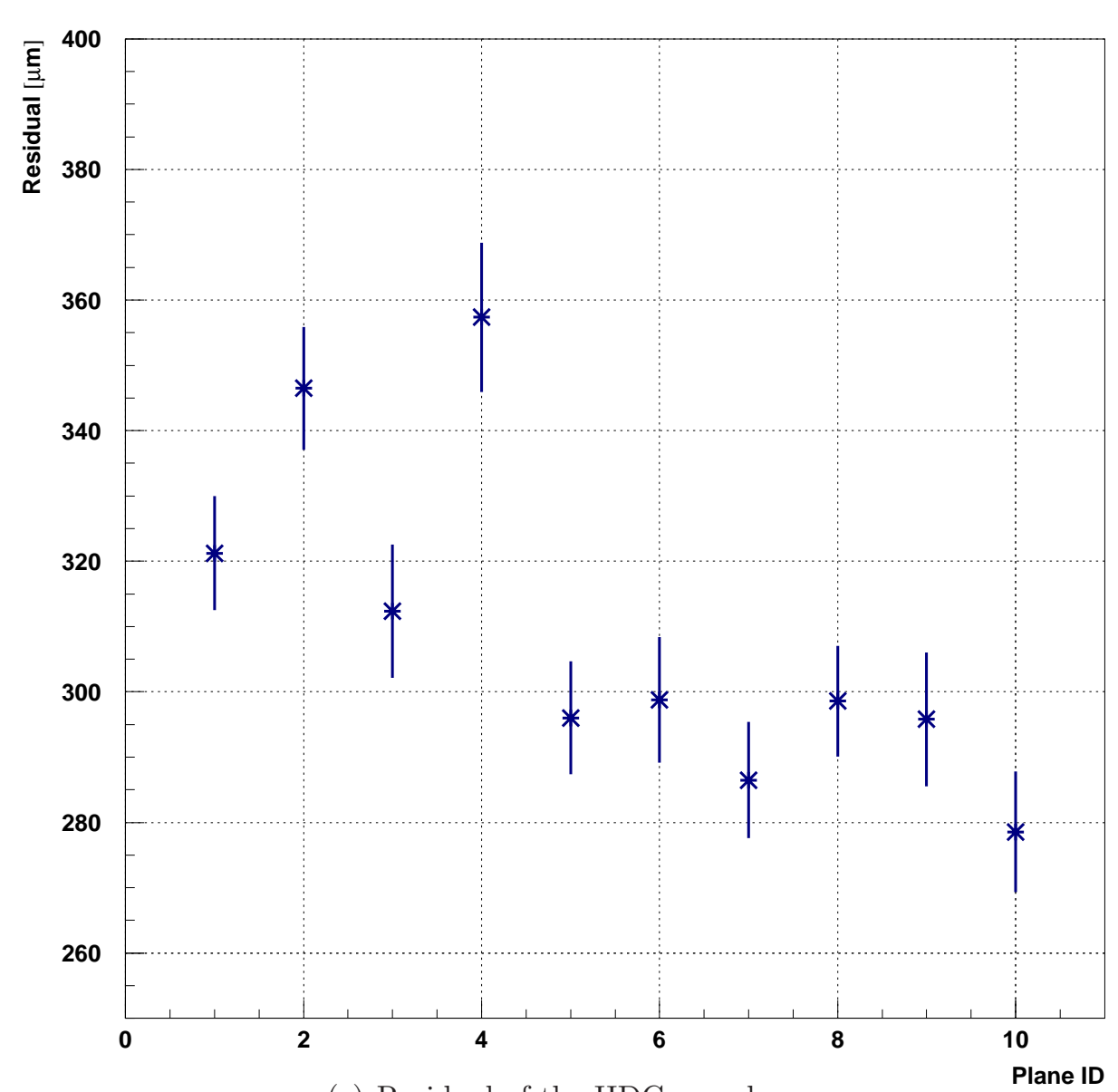

(c) Residual of the HDC per plane

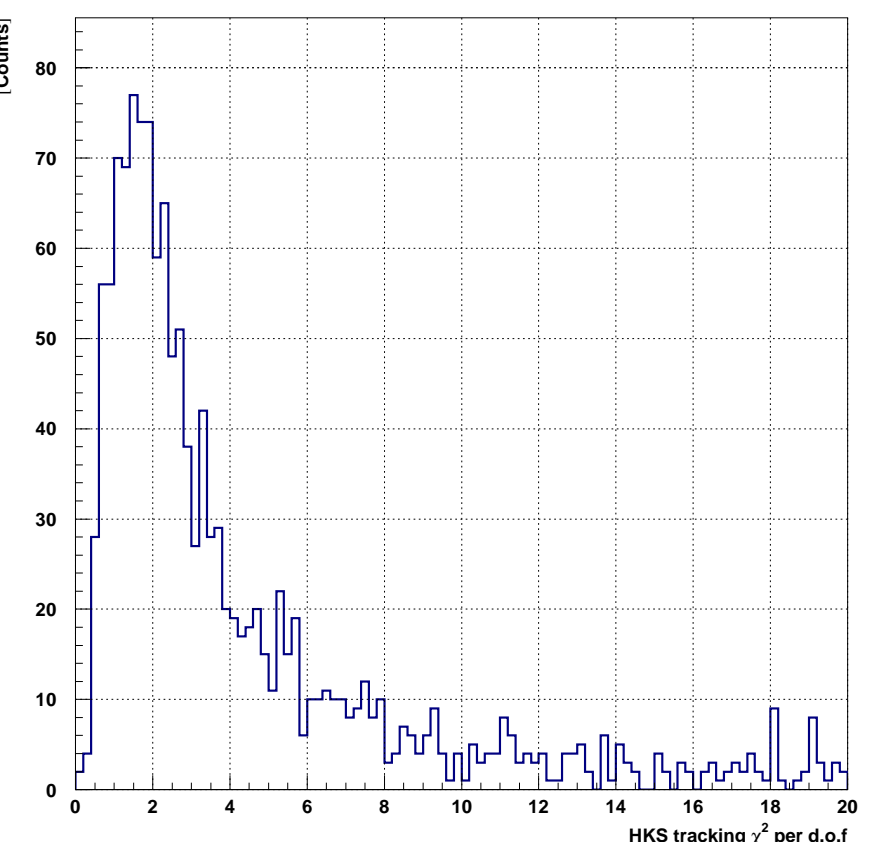

(b) HKS tracking $\chi^{2}$ per d.o.f 
$(p)$ as follows:

$$
\beta_{x}=\frac{p}{\sqrt{p^{2}+m_{x}^{2}}}
$$

Since the measured momenta in the HKS are more than $1 \mathrm{GeV} / c$ for positrons, $\beta_{e^{+}}$can be

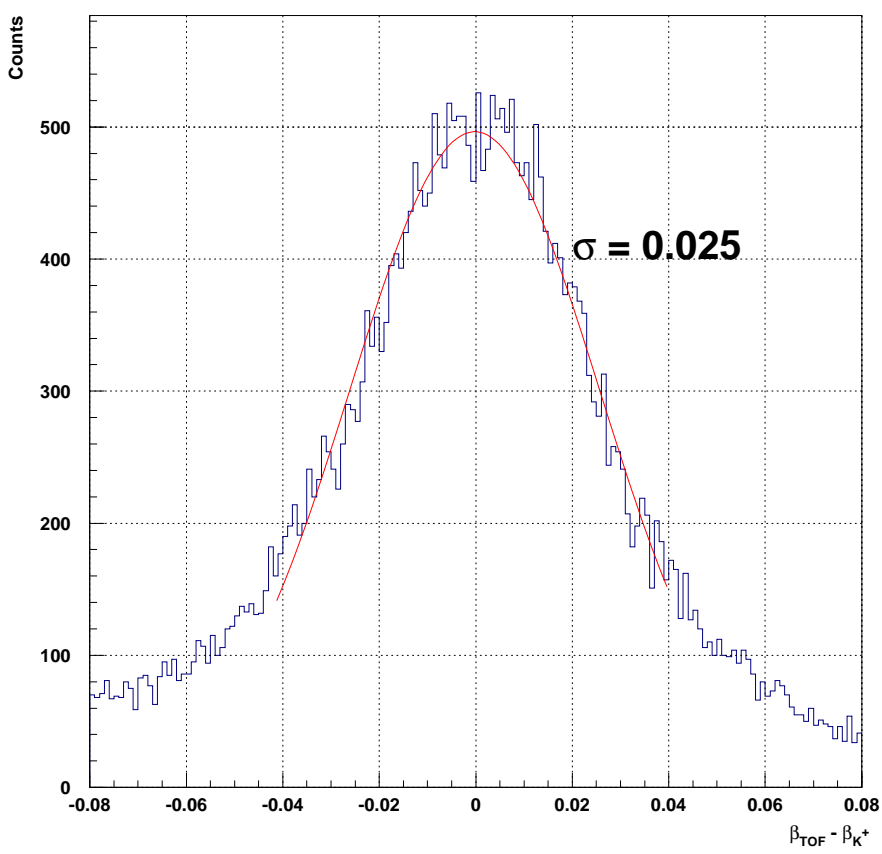

Figure 4.3: A typical particle velocity $\beta$ resolution was $0.025(\sigma)$.

treated as 1 . The $\beta_{\mathrm{TOF}}-\beta_{\mathrm{K}^{+}}$spectrum and a residual of RF (a Radio Frequency signal which is provided from the $499 \mathrm{MHz}$ of RF cavity frequency on the accelerator site) time and HKS target time correlation are represented in Fig. 4.4 (left and right), respectively.

The RF time is pre-scaled by a factor of 8 so that the rate can be handled by a TDC. The HKS target time is the particle emission time at the target point calculated by the HKS path length, the TOF calculation and the kaon mass.

Here, let us define

$$
\text { Target time }=(\text { RF time })-(\text { HKS target time }),
$$

for the convenience of the following discussion.

In the $\beta_{\mathrm{TOF}}-\beta_{\mathrm{K}^{+}}$spectra, kaons appear on the center of the distribution, and neighboring protons and pions are also identified even at the on-line analysis level.

Taking into account

- the $\beta$ difference between a proton and kaon in an energy region around $1.2 \mathrm{GeV}$ is more than 0.1 ,

- the typical $\beta$ resolution in the present TOF measurement is less than 0.025 ,

the protons are excluded using a window of $\beta_{\mathrm{TOF}}-\beta_{\mathrm{K}^{+}}>0.07(\sim 3 \sigma)$ as shown in Fig. 4.4 (left). 

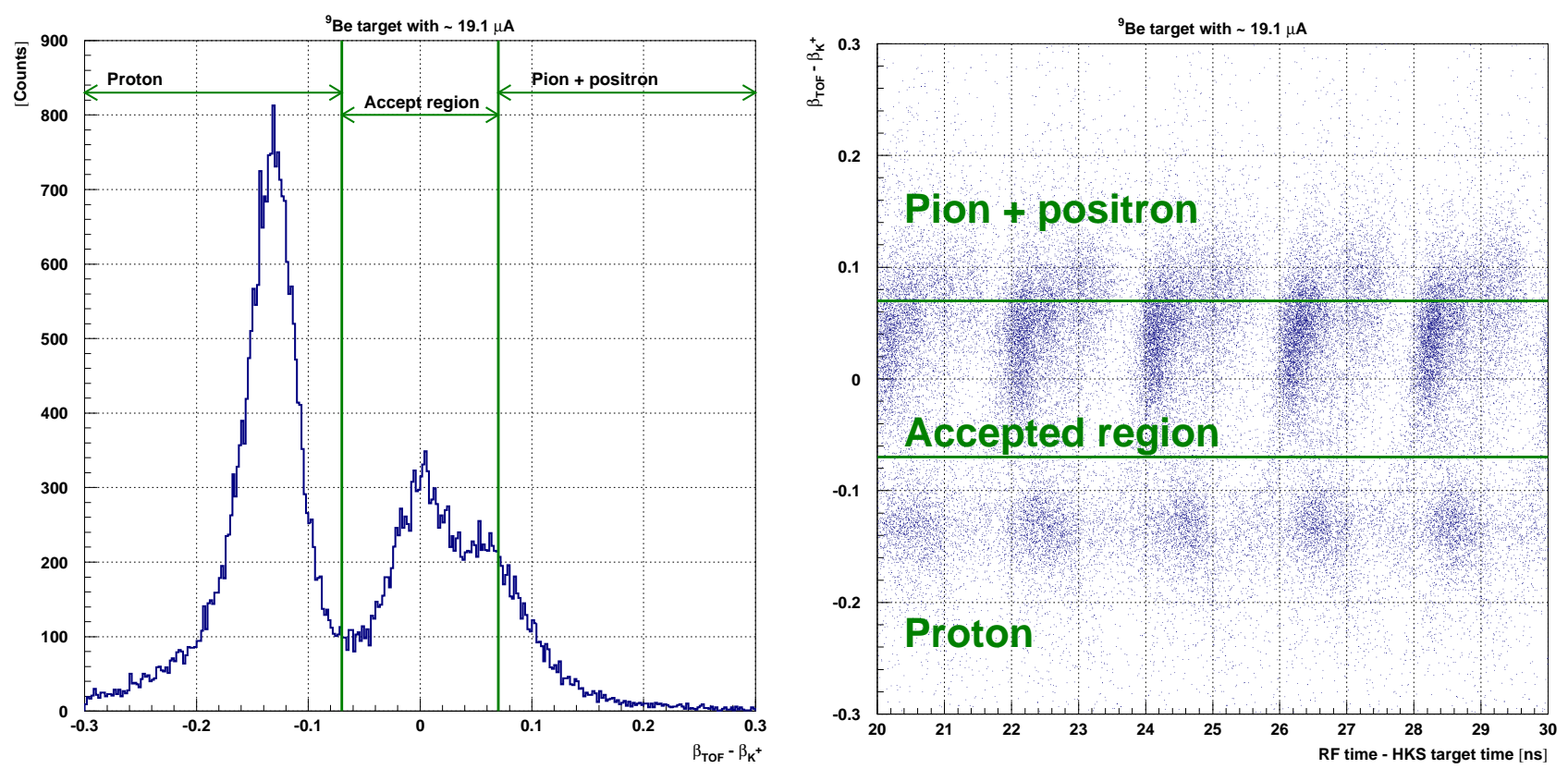

Figure 4.4: Particle velocity $\left(\beta_{\mathrm{TOF}}-\beta_{\mathrm{K}^{+}}\right)$distribution for the HKS arm (left) and PID with a scatter plot of the particle velocity and the target time (right).

\section{Proton cut}

As we discussed in Chapter 3, some protons were rejected with the water Cerenkov counter (WČ ) at the hardware level in order to suppress the trigger rate associated with proton as much as possible.

However, a further strict proton suppression is required because :

1) the threshold of the WČ was set at a safe level to avoid kaon overkill,

2) the number of photoelectrons was decreased due to radiation damage.

Therefore, the HKS $\beta$ cut, normalized WČ number of photoelectron cut and target time cut are required at the off-line analysis level to reject the remaining protons.

A scatter plot of normalized WČ number of photoelectrons and $\beta_{\mathrm{TOF}}-\beta_{\mathrm{K}^{+}}$is shown in Fig. 4.5 .

A scatter plot of the target time and $\beta_{\mathrm{TOF}}-\beta_{\mathrm{K}^{+}}$with offline $\mathrm{AC}$ and $\mathrm{WC}$ cuts and a $\beta_{\mathrm{TOF}}-\beta_{\mathrm{K}^{+}}$ cut is represented in Fig. 4.6 (left). Kaon events are aligned in the figure and the other particle events are not due to the misuse of the particle mass at the reconstruction. Since some amount of protons still survive after offline Čerenkov and $\beta_{\mathrm{TOF}}-\beta_{\mathrm{K}^{+}}$cuts, additional target time cuts are required to exclude the remaining protons.

Finally kaon events are identified within the solid box described in Fig. 4.6 (left). The target time spectrum with off-line Čerenkov cuts and $\beta_{\mathrm{TOF}}-\beta_{\mathrm{K}^{+}}$cut i.e., projection of the Fig. 4.6 (left) is shown in Fig. 4.6 (right).

The resolution of the time spectrum for true kaon events is 130 ps in sigma. Therefore, each peak in Fig. 4.6 is selected within 1 ns to reject protons.

Eventually, the target time cut is expressed as a set of :

$\mid($ Target time $)-($ Time centers in each peak $) \mid<0.5 \mathrm{~ns}$. 


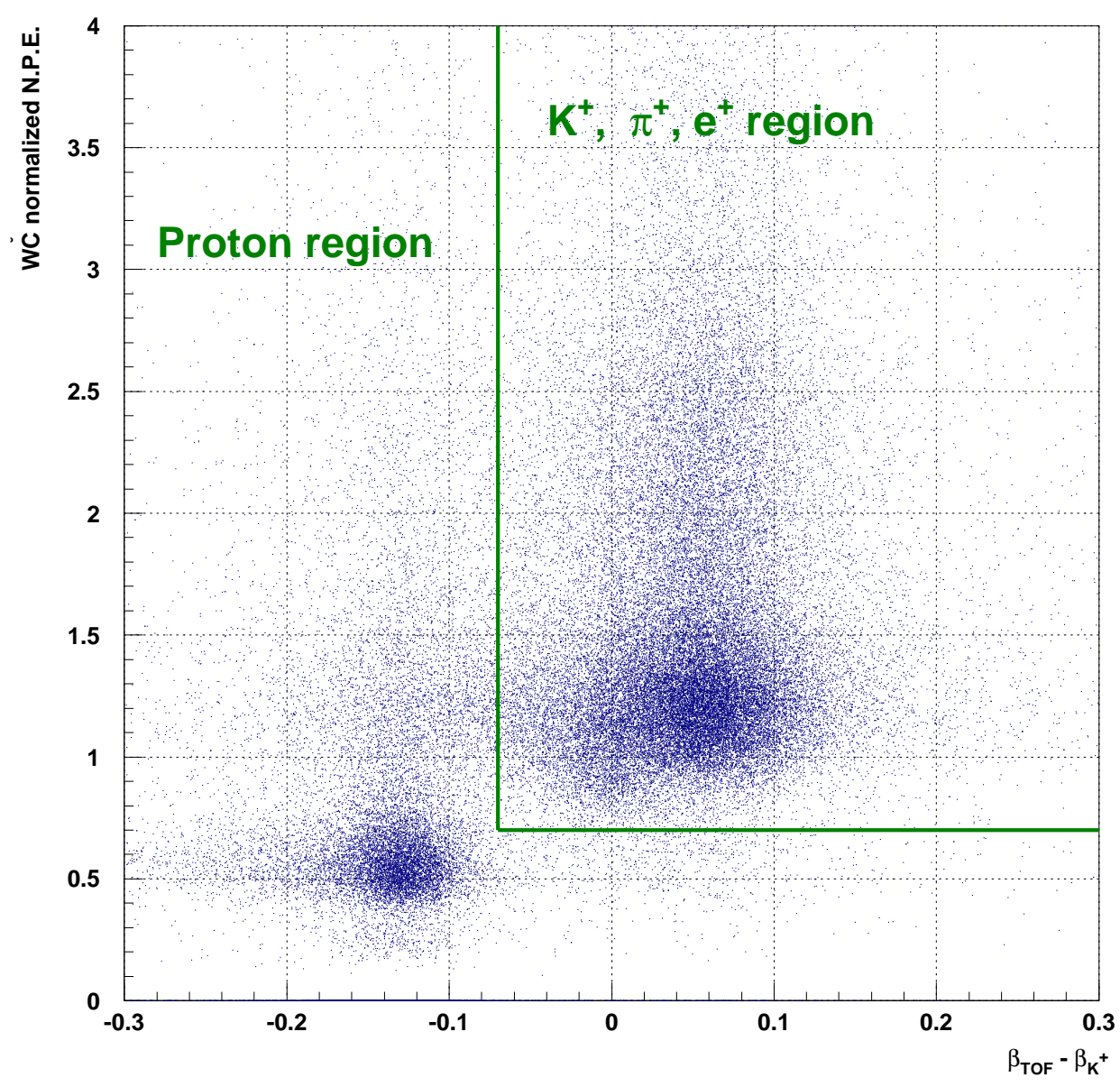

Figure 4.5: A scatter plot of normalized WČ number of photoelectrons vs. $\beta_{\mathrm{TOF}}-\beta_{\mathrm{K}^{+}}$for proton rejection. 
The effect of the target time cut is described in Fig. 4.7.
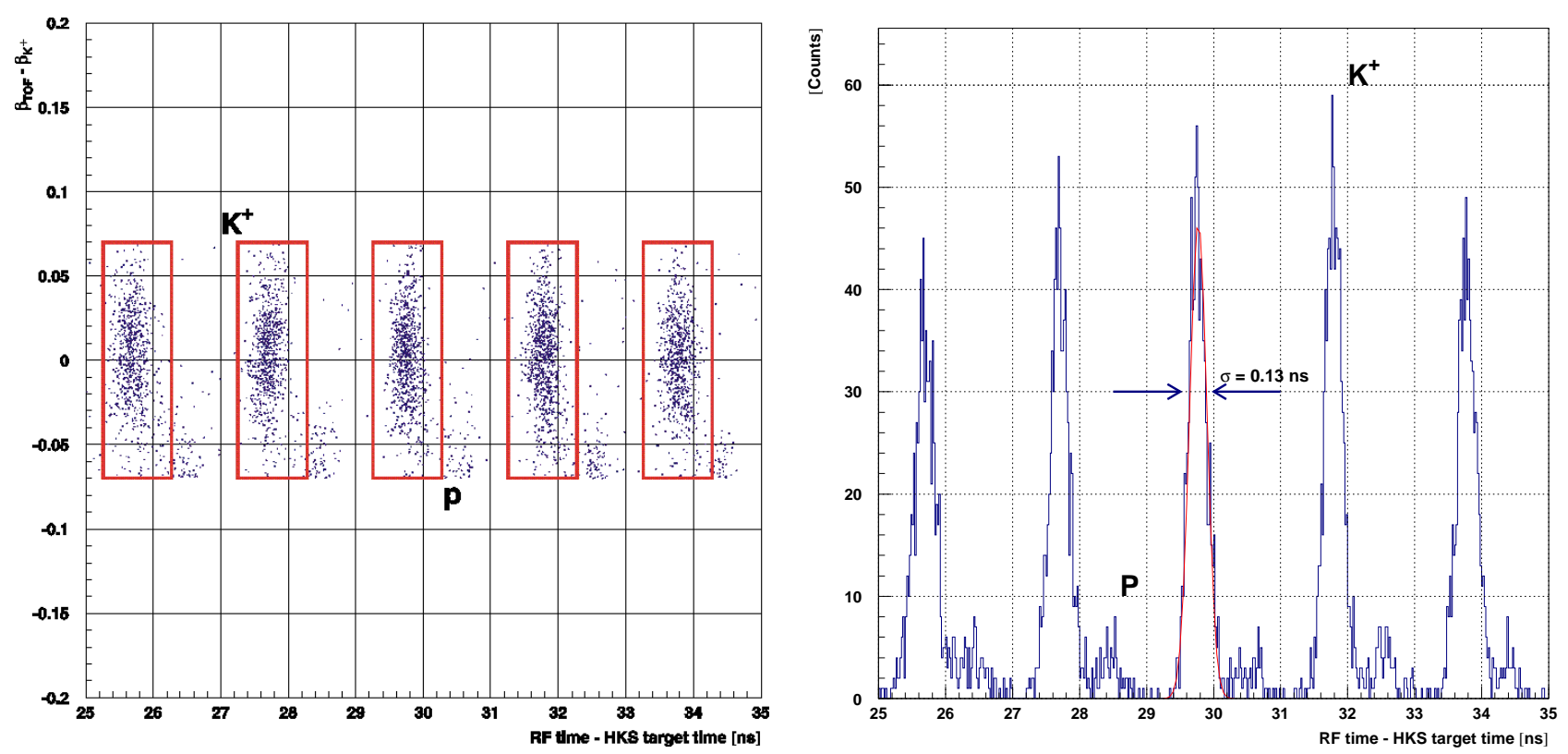

Figure 4.6: A scatter plot of the target time and $\beta_{\mathrm{TOF}}-\beta_{\mathrm{K}^{+}}$(left) and the target time spectrum (right)

\section{Pion cut}

Pion events are rejected with a signal veto from aerogel Čerenkov counters (A $\check{C}$ ) and the $\beta_{\mathrm{TOF}}-\beta_{\mathrm{K}^{+}}$cuts.

First, a loose AC cut $\left(N P E_{\mathrm{AC}}<50\right)$ was applied at the on-line level in order to avoid unexpected kaon loss, then a tight $\mathrm{AC}$ cut $\left(N P E_{\mathrm{AC}}<\sim 3\right)$ and $\left|\beta_{\mathrm{TOF}}-\beta_{\mathrm{K}^{+}}\right|<\sim 0.07$ are applied for precise kaon selection in the off-line analysis as shown in Fig. 4.8. 


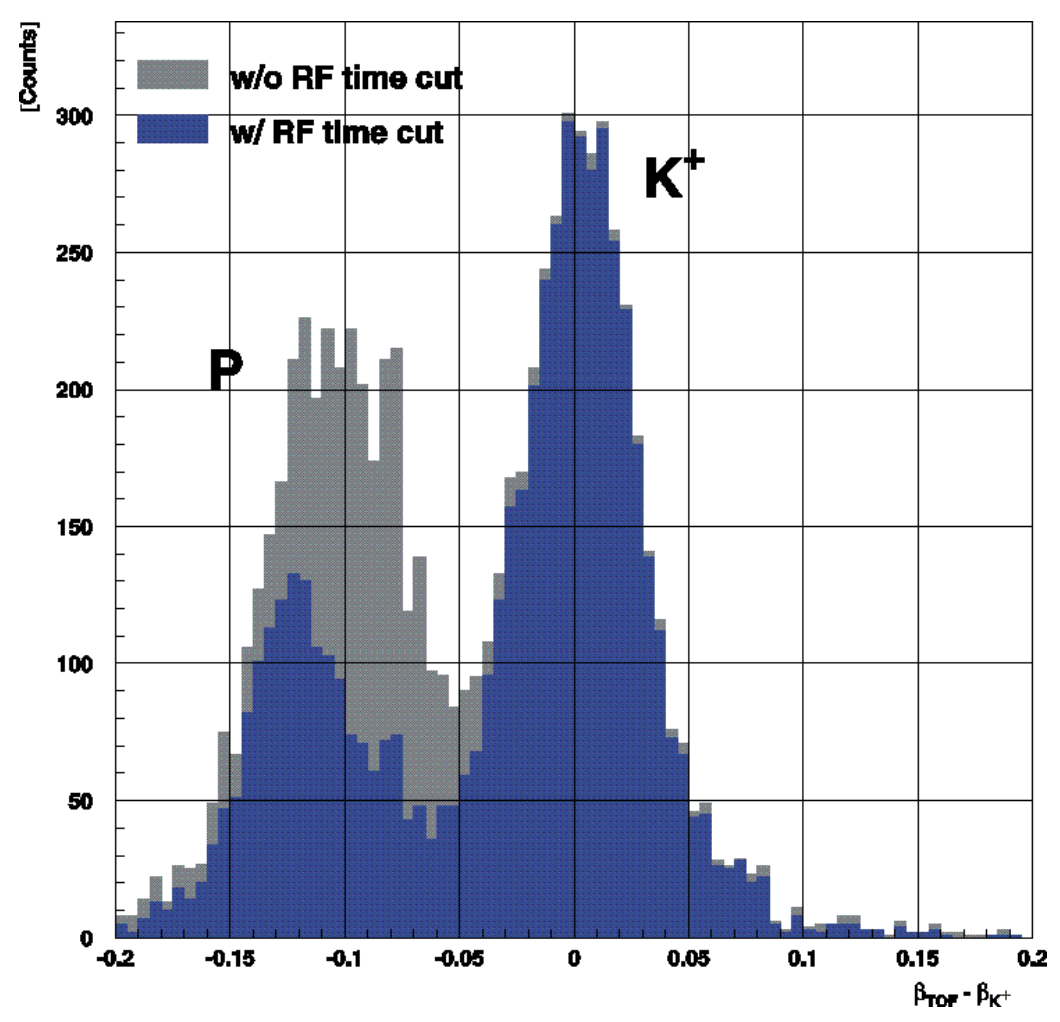

Figure 4.7: The target time cuts effect for kaon selection with $\beta_{\mathrm{TOF}}-\beta_{\mathrm{K}^{+}}$spectrum.

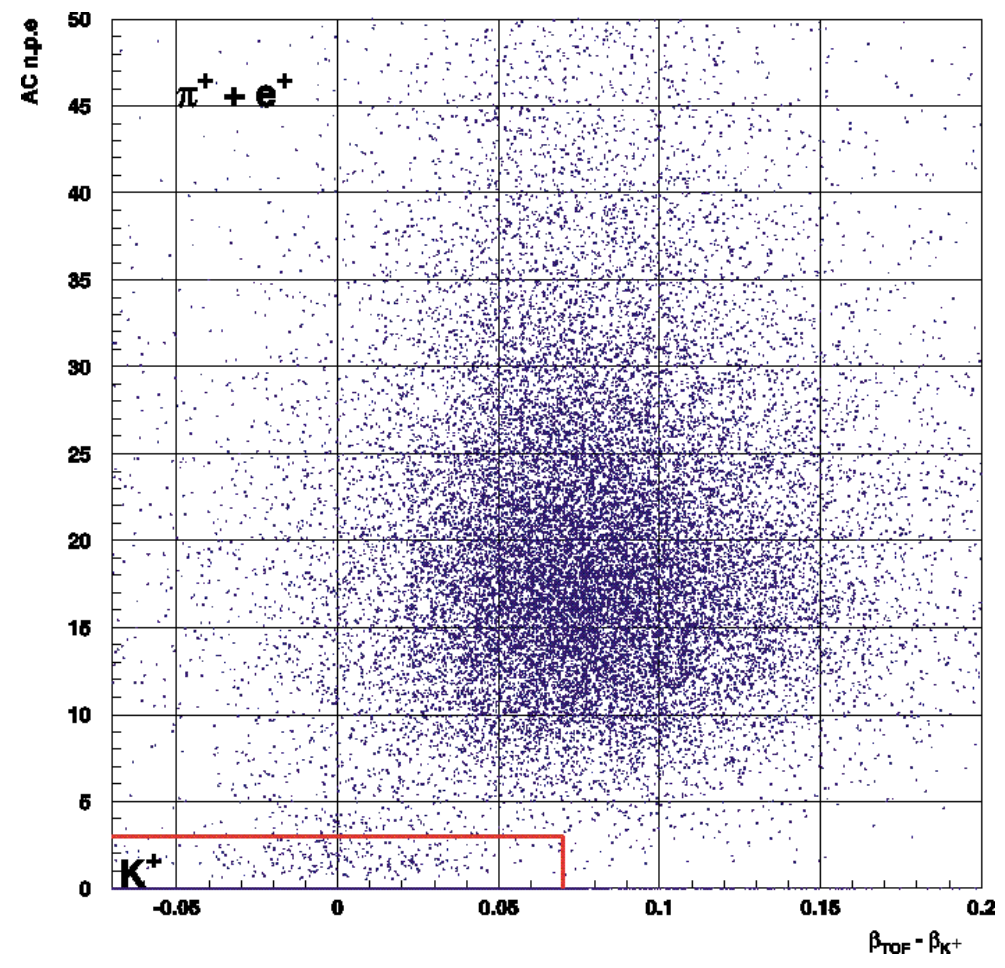

Figure 4.8: Scatter plot of $\mathrm{AC}$ number of photoelectrons vs. $\beta_{\mathrm{TOF}}-\beta_{\mathrm{K}^{+}}$for pion and positron rejection. 

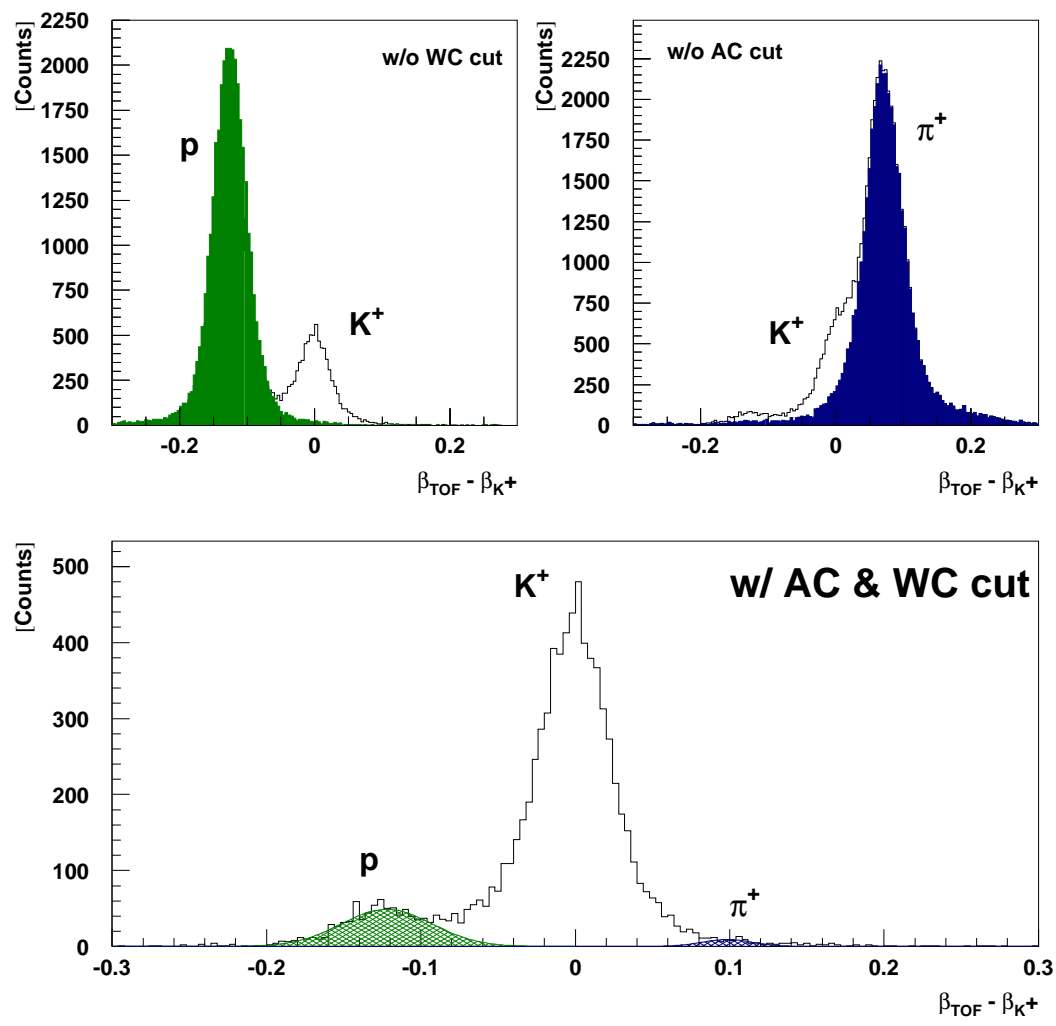

Figure 4.9: $\beta_{\mathrm{TOF}}-\beta_{\mathrm{K}^{+}}$spectra with/without Čerenkov cut.

\subsection{Scattered electron measurement in Enge}

Electrons are the dominant scattered particles in the Enge. Position and angle at the focal plane for each event are measured by the hit position of the Enge drift chamber (EDC), and timing information is measured by two layers of the Enge hodoscopes (EHODO1 and EHODO2). The time information of each hodoscope is adjusted by a pulse-height correction and any offset associated with cable length and hit position. Electrons are identified by a coincidence between EDC and EHODOs. More than 8 of 10 layers hits are required for the EDC and 2 layers for EHODOs.

Scatter plots of hit pattern of the EHODO (layer1 : upper and layer2 : lower) and the horizontal coordinate at the Enge focal plane are represented in Fig. 4.10 .

Some amount of events with counter IDs of half integers, indicate to pass through neighboring two scintillators for each layer. The ratio of such kind of events were estimated to be $2 \sim 4 \%$ for each layer in Fig.4.10. 

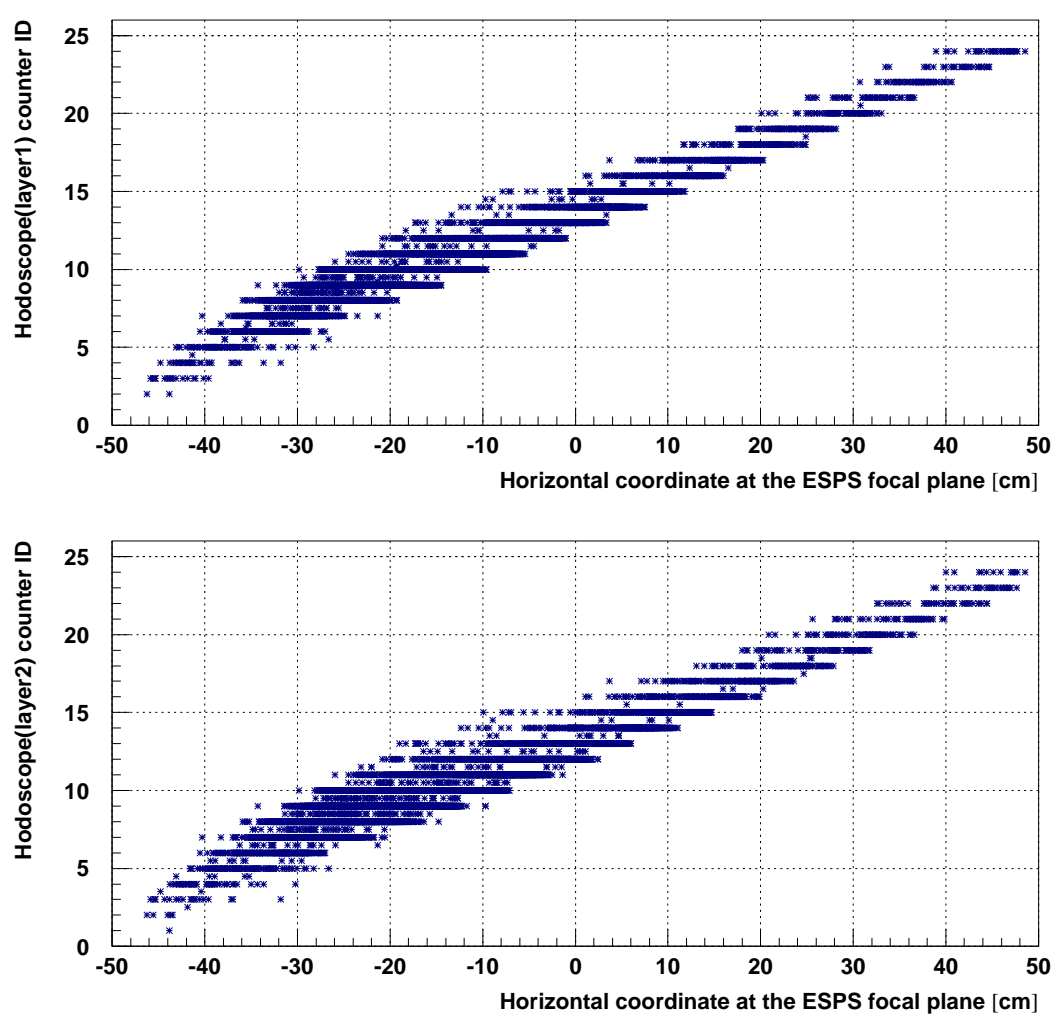

Figure 4.10: A geometrical correlation between horizontal coordinate at the Enge focal plane and the Enge hodoscopes.

\subsection{Two arms coincidence}

The true $\left(\mathrm{e}, \mathrm{e}^{\prime} \mathrm{K}^{+}\right)$reaction is identified by the coincidence time $\left(t_{\text {coin }}\right)$ which is traced back from both HKS and Enge focal planes to the target point as described in Eqn. 4.4,

$$
t_{\text {coin }}=t_{\mathrm{HKS}}(\operatorname{tar})-t_{\text {Enge }}(\operatorname{tar})
$$

where $t_{\mathrm{HKS}}(\operatorname{tar})$ and $t_{\text {Enge }}(\mathrm{tar})$ are the target times reconstructed from the Enge/HKS focal planes to the target point. Fig. 4.11 shows a $\beta_{\mathrm{TOF}}-\beta_{\mathrm{K}^{+}}$VS. $t_{\text {coin }}$ correlation (upper) and a coincidence time $\left(t_{\text {coin }}\right)$ distribution.

Since a beam bunch is delivered to Hall C every $2 \mathrm{~ns}$, the spectrum of the $t_{\text {coin }}$ also shows the beam structure. In Fig. 4.11 (lower), true (+ accidental) coincidence events (true peak) are included in one of the bunches with a width of $1 \mathrm{~ns}$ (sigma) while accidental random coincidence events (accidental peak) appear in the all bunches.

In this analysis, the number of true coincidence events is derived from the number of counts in the true peak subtracted by the average number of accidental counts 4 neighboring accidental peaks.

\section{Mixed event analysis for the accidental background}

The hypernuclear cross sections are derived by subtracting the accidental background as described above. The statistical fractuation, which is produced by this procedure, should be minimized as much as possible. 

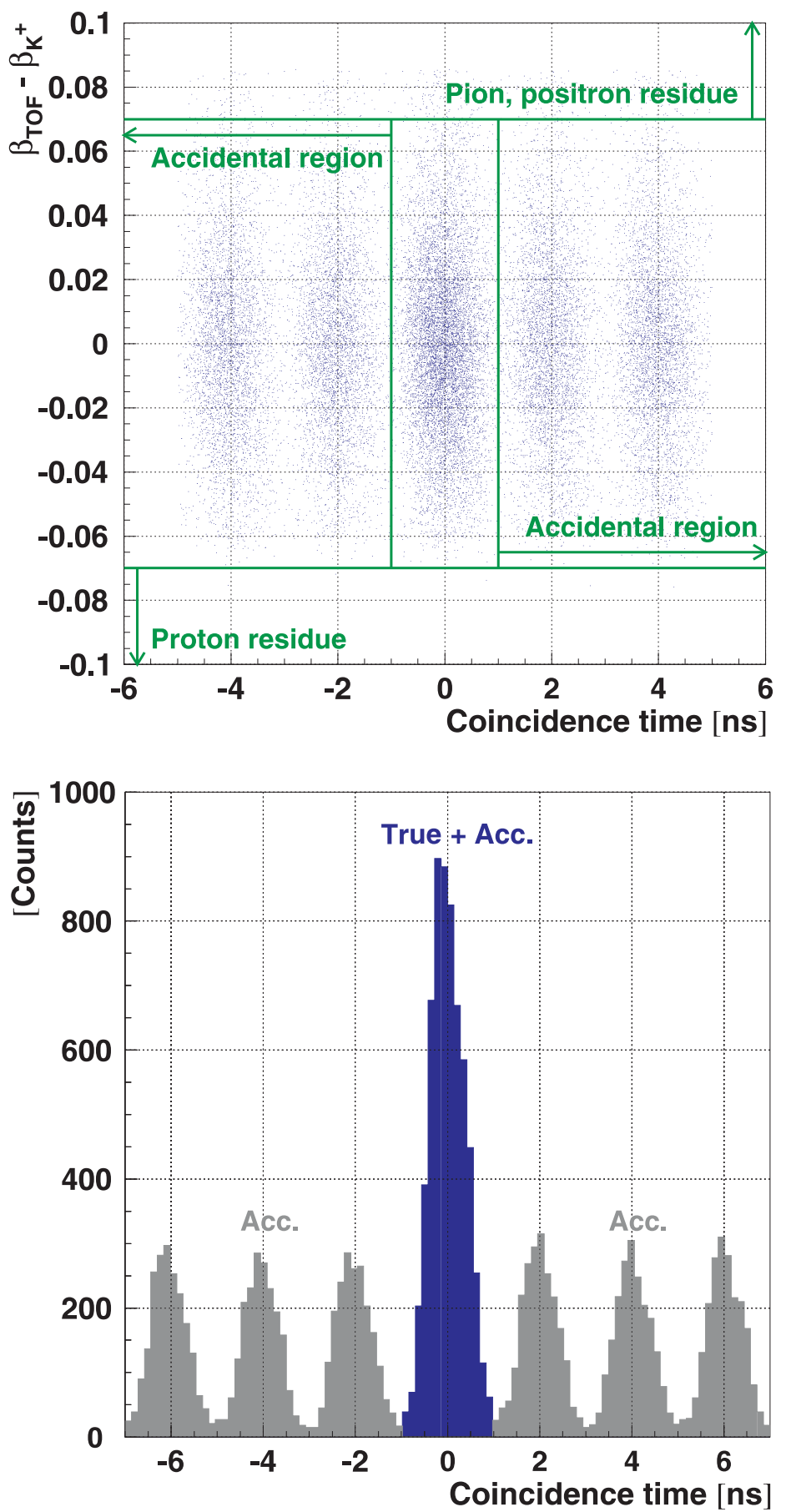

Figure 4.11: $\beta_{\mathrm{TOF}}-\beta_{\mathrm{K}^{+}}$VS. coincidence time correlation (upper) and coincidence time spectrum (lower) 
In order to do so, a fluctuation (mixed event analysis) is introduced and applied to the accidental background events.

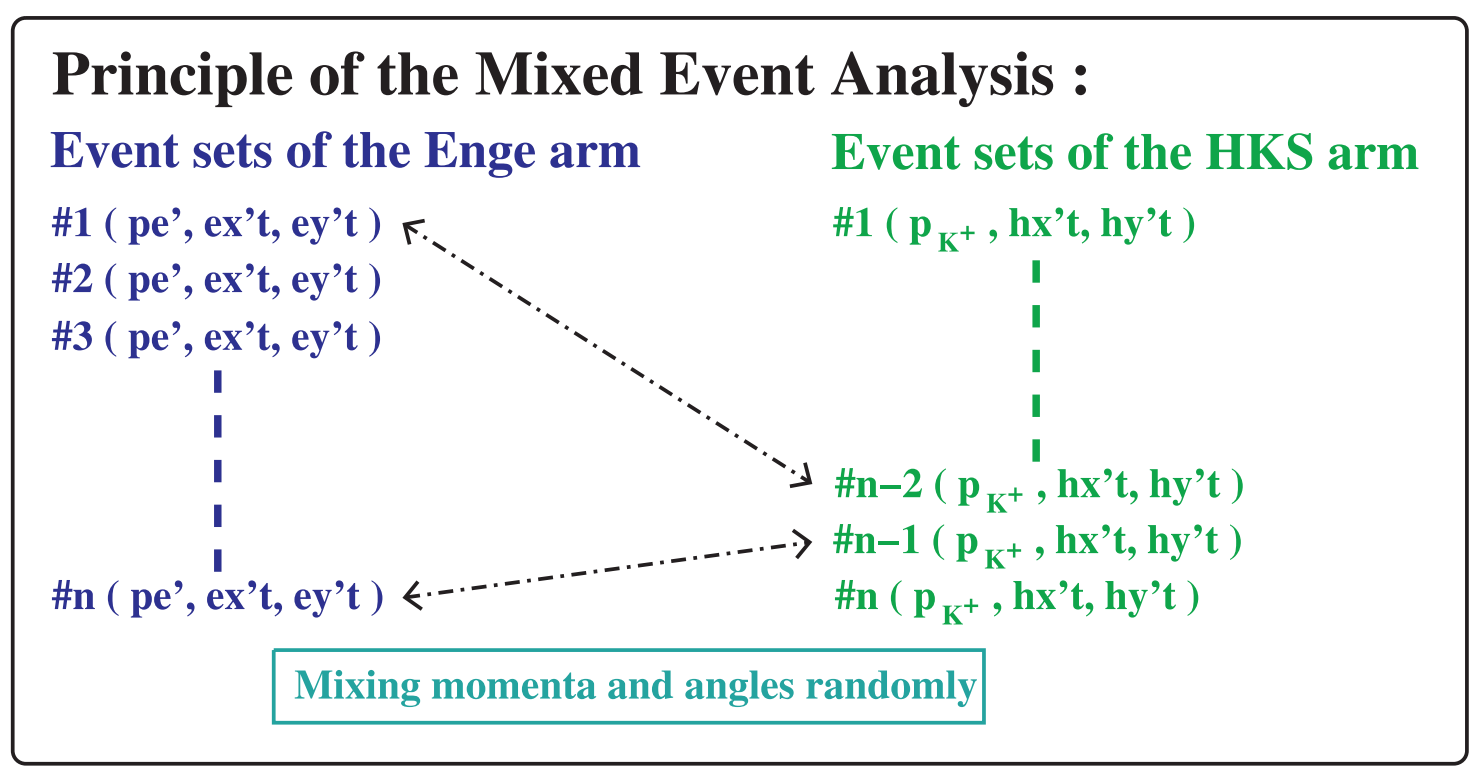

Figure 4.12: Principle of the mixed event analysis for the accidental background events.

The principle of the mixed event analysis is described in Fig. 4.12.

First, four bunches of the explicit accidental coincidence events (see also Fig. 4.11) are selected with :

$$
1[\mathrm{~ns}]<\mid \text { Coincidence time } \mid<5[\mathrm{~ns}]
$$

These selected accidental coincidence events contain particle information of the momenta $\left(p_{i}\right.$, $\left.i=\mathrm{e}^{\prime}, \mathrm{K}^{+}\right)$and emission angles $\left(i x^{\prime} t, i=\mathrm{e}, \mathrm{h}\right)$ at the target point of the Enge and HKS, respectively for each event. The mixed event analysis replaces event set combinations free from event ID. Therefore, as far as using the original accidental event sets $(n)$, the statistics of the accidental background is $n / 4$ as described as a hatched region of $\Lambda$ missing mass spectrum in Fig. 4.13 (a). On the other hand, using the mixed event analysis, the statistics of that is increased up to $n^{2} / 4$, and produces a smooth accidental background shape as shown in Fig. $4.13(\mathrm{~b})$. 


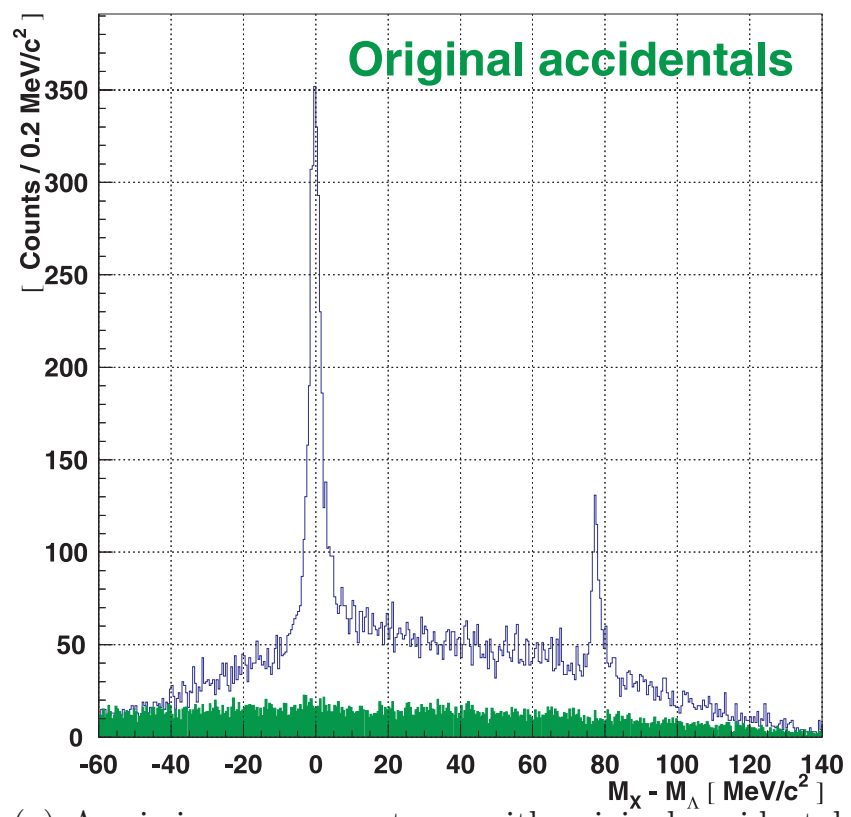

(a) $\Lambda$ missing mass spectrum with original accidental coincidence.

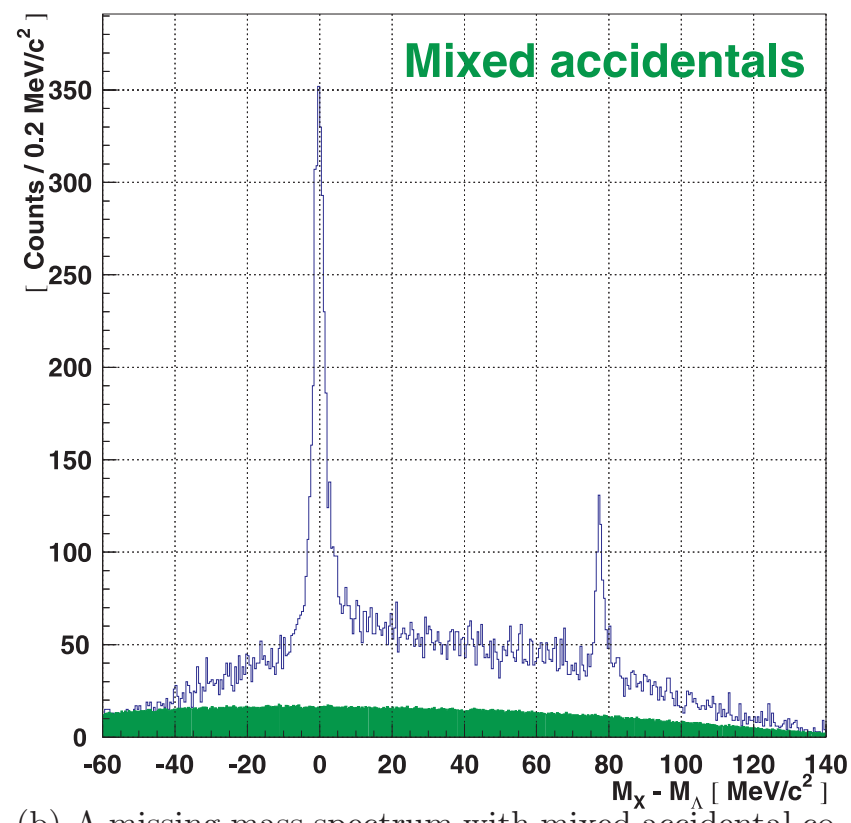

(b) $\Lambda$ missing mass spectrum with mixed accidental coincidence.

Figure 4.13: Effectiveness of the mixed event analysis on the $\Lambda$ missing mass spectra.

\subsection{Emission angle and momentum calibration}

A missing mass of a $\Lambda$ hypernuclear mass $M_{\mathrm{HY}}$ can be extracted by energy and momentum conservation law using a total of 6 variables : the momenta $\left(p_{\mathrm{K}^{+}}, p_{\mathrm{e}^{\prime}}\right)$ and scattering angles at the target point $\left(\theta_{\mathrm{K}^{+}}, \phi_{\mathrm{e}^{\prime}}, \theta_{\mathrm{K}^{+}}, \phi_{\mathrm{e}^{\prime}}\right)$ of the kaon and scattered electron defined with center of mass coordinates as shown in Fig. 4.14.

The full expression of $M_{\mathrm{HY}}$ is written as,

$$
\begin{aligned}
M_{\mathrm{HY}}^{2} & =\left(E_{\mathrm{e}}+M_{A}-E_{\mathrm{K}^{+}}-E_{\mathrm{e}}\right)^{2} \\
& -\left(p_{\mathrm{e}}^{2}+p_{\mathrm{e}^{\prime}}^{2}+p_{\mathrm{K}^{+}}^{2}-2 p_{\mathrm{e}} p_{\mathrm{e}^{\prime}} \cos \theta_{\mathrm{ee}^{\prime}}-2 p_{\mathrm{e}} p_{\mathrm{K}^{+}} \cos \theta_{\mathrm{eK}^{+}}+2 p_{\mathrm{e}} p_{\mathrm{K}^{+}} \cos \theta_{\mathrm{e}^{\prime} \mathrm{K}^{+}}\right)
\end{aligned}
$$

where $E_{x}$ are the total energies of $x=\mathrm{e}, \mathrm{e}^{\prime}, \mathrm{K}^{+}$, and $A$, the target nucleus.

Since the quantities we can obtain experimentally are the incident angles and positions on the focal plane for both the Enge and HKS arms, both momentum and emission angles at the target point are reconstructed with transfer matrices which are described as 6th order polynomials with focal plane quantities

$$
X_{t}=M_{\mathrm{F} 2 \mathrm{~T}} X_{f}=\sum_{\alpha+\beta+\gamma+\eta=1}^{6} C(\alpha, \beta, \gamma, \eta) x_{f}^{\alpha} x_{f}^{\prime \beta} y_{f}^{\gamma} y_{f}^{\prime \eta},
$$

where $X_{t}$ represents particle momentum $d p\left(d p=\left(p-p_{c}\right) / p_{c} \times 100\right)$ and emission angles at the target point $x_{t}^{\prime}$ and $y_{t}^{\prime}, X_{f}$ means focal plane coordinates and angles $x_{f}, x_{f}^{\prime}, y_{f}$ and $y_{f}^{\prime}$ for the Enge and HKS. And the expansion coefficients $C_{n}$ mean the backward transfer matrix $\left(M_{\mathrm{F} 2 \mathrm{~T}}\right)$ elements defined from the focal plane to the target point. 


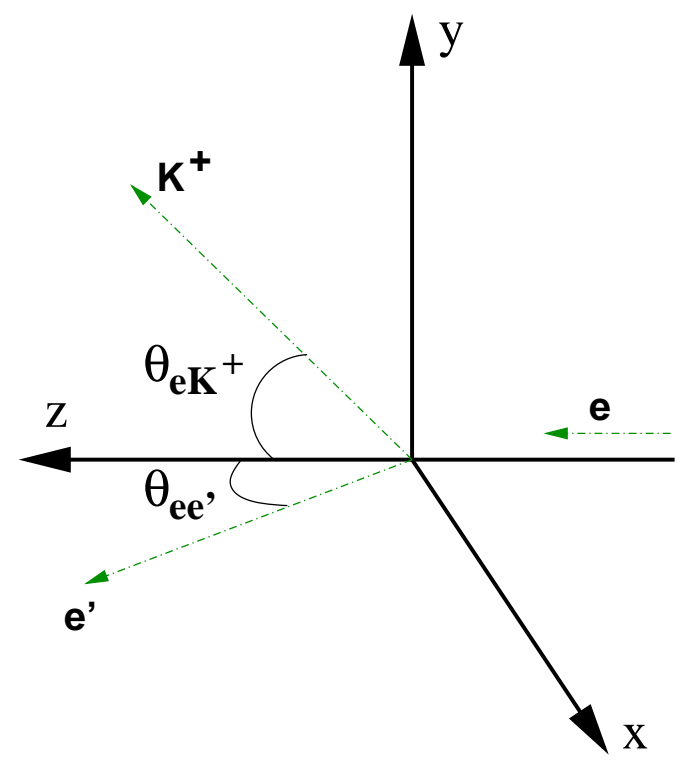

Figure 4.14: Momenta and emission angles definition with center of mass coordinates for mass spectrum calculation.

\subsubsection{Analysis overview and flow chart}

A general flow chart for missing mass reconstruction is described in Fig. 4.15.

Basic optics for the Enge and HKS are designed with the program RAYTRACE [60,61] and the Monte Carlo simulation software GEANT, respectively in order to extract the first approximation of the transfer matrix $\left(M_{\mathrm{F} 2 \mathrm{~T}}\right)$. Magnetic field descriptions for each spectrometer have been calculated with TOSCA, a magnetic field calculation software.

During the experiment, sieve slit (S.S.) plates were used for the further optics tuning.

The S.S. plates are made of a tungsten based alloy located the entrance of the Enge and the first quadrupole magnet of the HKS arm.

Fig. 4.18 (upper, lower) show the reconstructed images at the entrance of Enge and HKS S.S. planes, respectively.

The $2.54 \mathrm{~cm}$ thick of the Enge S.S. has $300.5 \mathrm{~cm} \phi$ slit holes with a $2 \mathrm{~cm}$ horizontal interval and a $1 \mathrm{~cm}$ vertical and a $11^{w} \times 4^{h} \mathrm{~cm}^{2}$ collimator.

The $5.08 \mathrm{~cm}$ thick of the HKS S.S. has $530.632 \mathrm{~cm}$ diameter holes with a $1.618 \mathrm{~cm}$ horizontal interval and $2.156 \mathrm{~cm}$ vertical spacing. One of the hole diameter is $0.318 \mathrm{~cm}$ and one hole is completely closed in order to break the symmetry making it possible to unambigously identify the slit holes on the focal plane.

1) Next, the basic $M_{\mathrm{F} 2 \mathrm{~T}}$ is applied to the S.S. run data to adjust the emission angle for each spectrometer and optimize the emission angle matrices which connect the focal plane to the target.

2) Missing mass is calculated with the updated emission angle matrices and the momentum matrix component of the basic $M_{\mathrm{F} 2 \mathrm{~T}}$. The mass scale was calibrated by adjusting the mass offset to obtain the well known masses; $\Lambda, \Sigma^{0}$ and ${ }_{\Lambda}^{12} \mathrm{~B}$ ground states.

3) Then momenta; $p_{\mathrm{e}}$, and $p_{\mathrm{K}^{+}}$are optimized by minimizing Eqn. 4.8, using non-linear least square fitting (NLS). A detailed description is in Section 4.5.3.

By iterating steps $1-3$, the reconstructed mass spectrum can be calibrated. The basic optics is modified several times in order to calibrate the definition of central scattering angles and central momenta for each arm. 


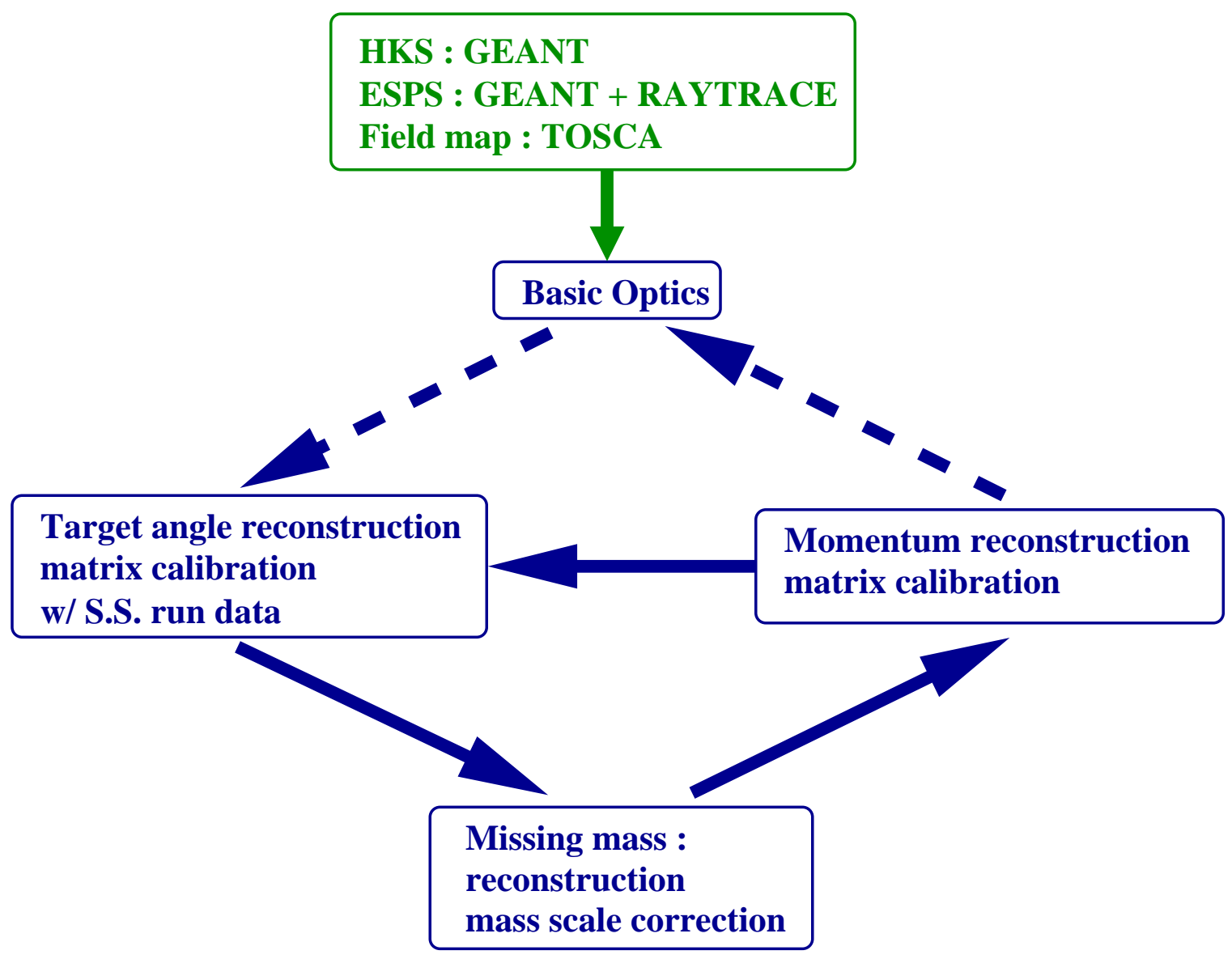

Figure 4.15: A flow chart for mass reconstruction. 

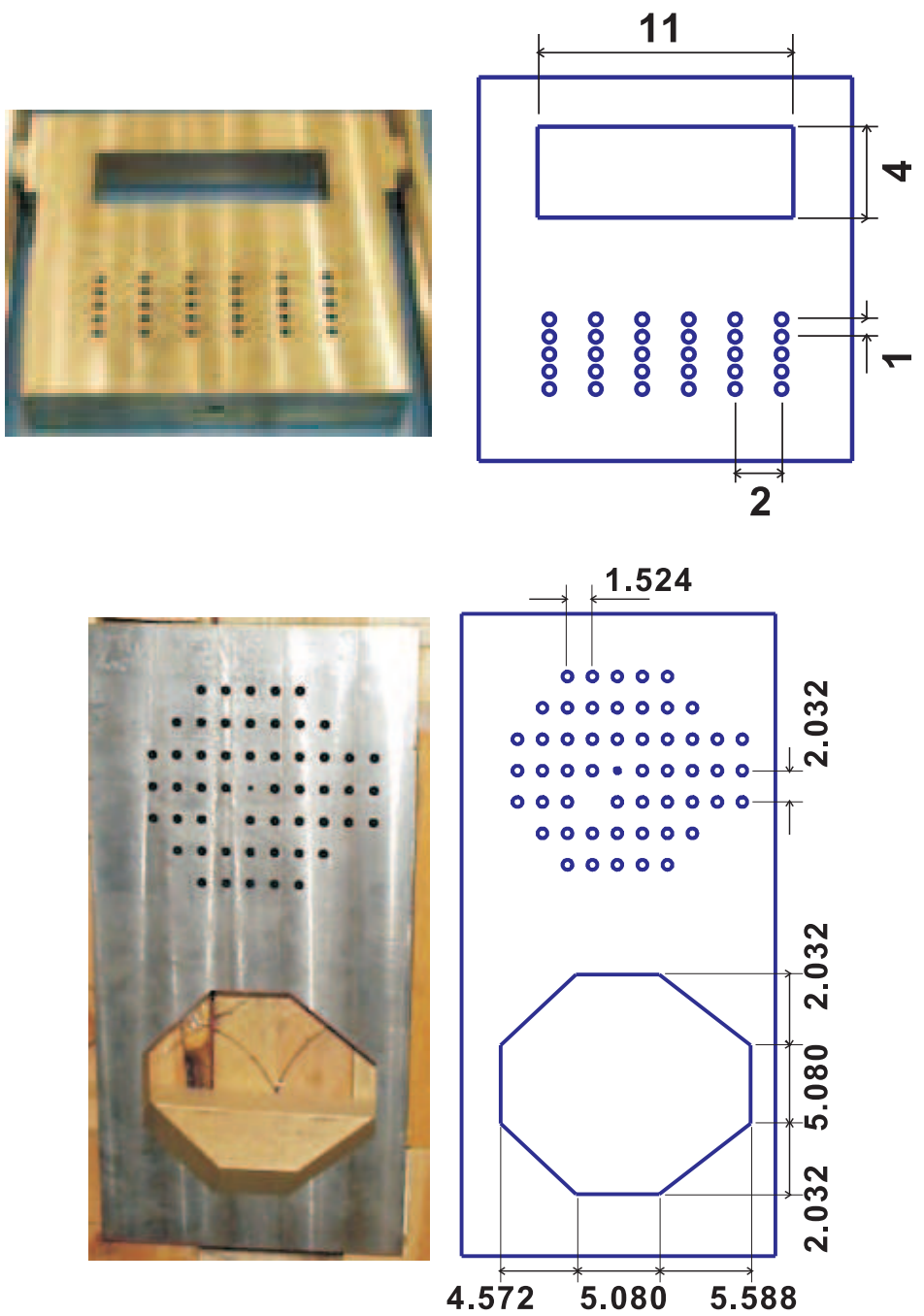

Figure 4.16: Enge (upper) and HKS (lower) collimator and sieve slit. Unit is cm. 
For the Enge, due to the tilt method, the geometrical description of the splitter and Enge split pole spectrometer connection is too complicated to represent with RAYTRACE, thus the description from target point to the sieve slit (S.S.) entrance plane has been modelled with GEANT.

\subsubsection{Emission angle calibration}

\section{ESPS/HKS Target Angle Calibration Procedure}

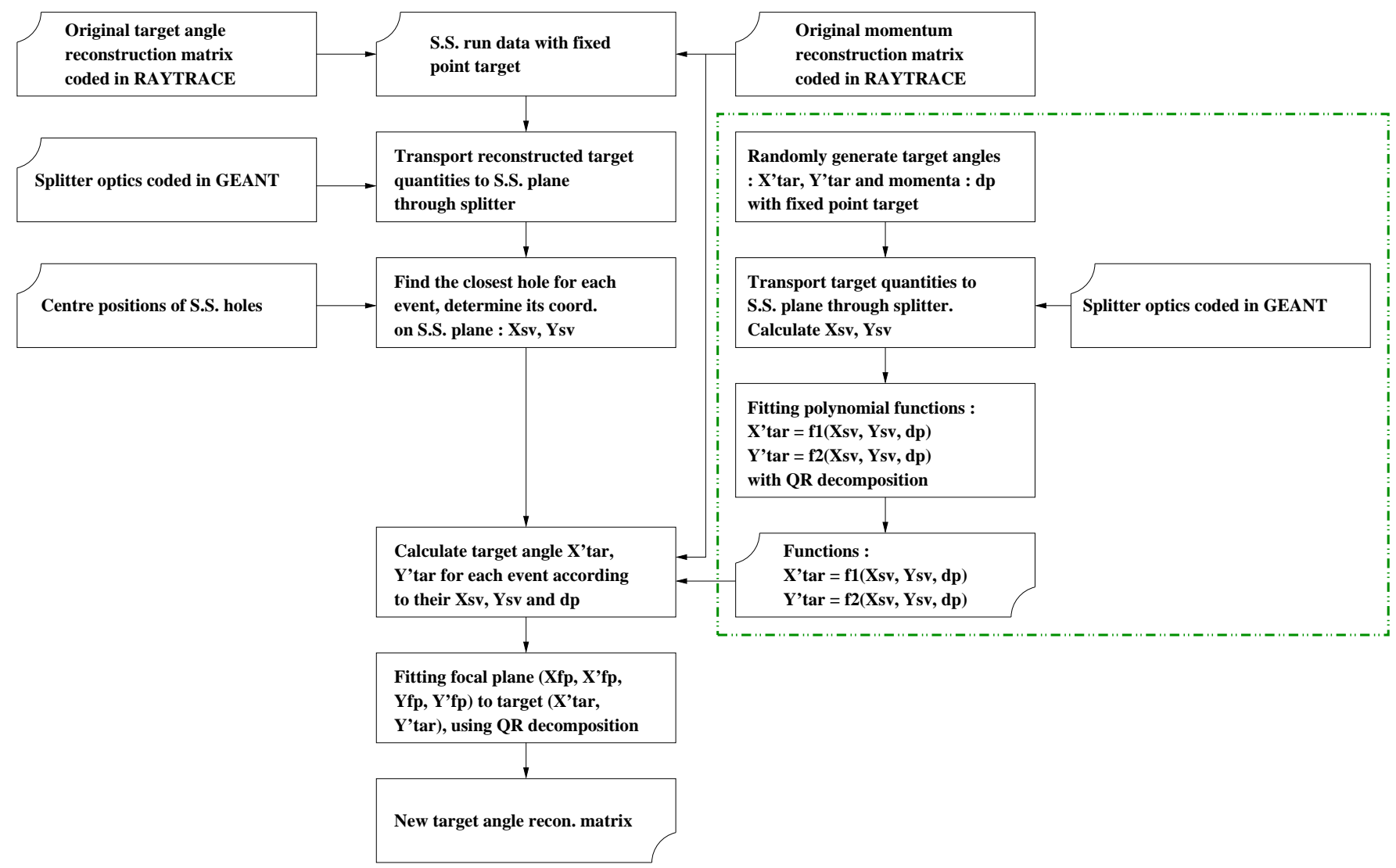

Figure 4.17: The flow chart for emission angle calibration procedure.

A flow chart for the emission angle calibration for both the Enge and HKS is shown in Fig. 4.17. First, the measured focal plane quantities of the individual Enge/HKS S.S. run data are transported to the emission angles with the basic matrices. Then the events with fixed target position are transported again to the S.S. which is placed between the splitter magnet and the entrance of the Enge split pole spectrometer for the electron arm, and the entrance of the first quadrupole magnet for the kaon arm as shown in Fig. 4.16, respectively.

After events are transported to the S.S. entrance plane, the center coordinates; $X_{\mathrm{sv}}$ and $Y_{\mathrm{sv}}$ are assigned according to the closest hole for each events as each event emitting one combination of the emission angles $\left(x_{t}^{\prime}, y_{t}^{\prime}\right)$ should be limited by the acceptance of each slit hole. Then applying the transfer matrices $\left(M_{\mathrm{S} 2 \mathrm{~T}}\right)$, which relates from the S.S. entrance and target point, emission angles can be corrected. Eventually, solving a linear equation which consists of $\left(x_{t}^{\prime}\right.$, 

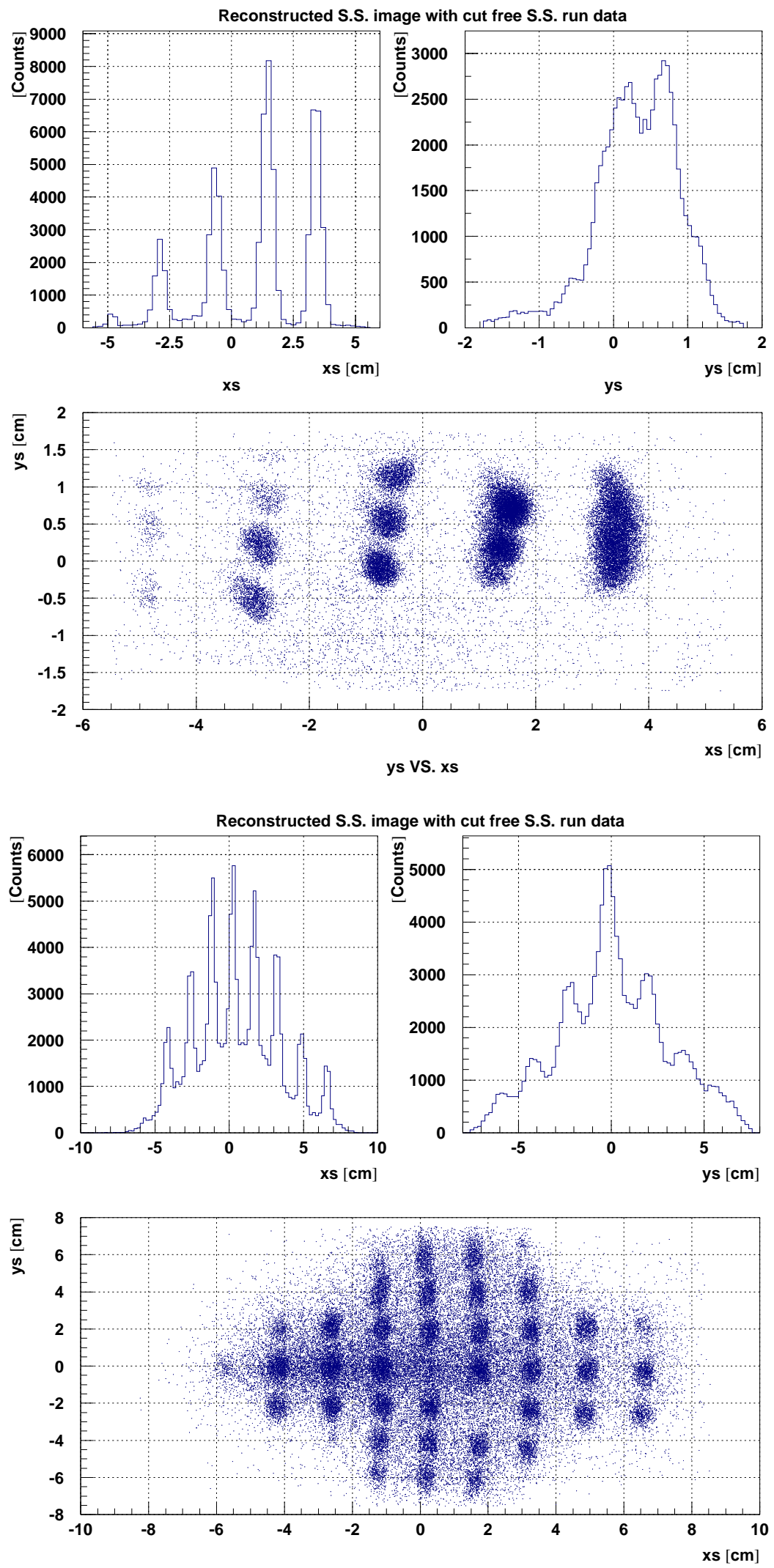

Figure 4.18: The reconstructed Enge (upper) and HKS (lower) S.S. images. 
$\left.y_{t}^{\prime}\right)$ and $\left(x_{f}, x_{f}^{\prime}, y_{f}, y_{f}^{\prime}\right)$ with the $\mathrm{QR}$ decomposition method, the emission angle reconstruction matrices can be optimized.
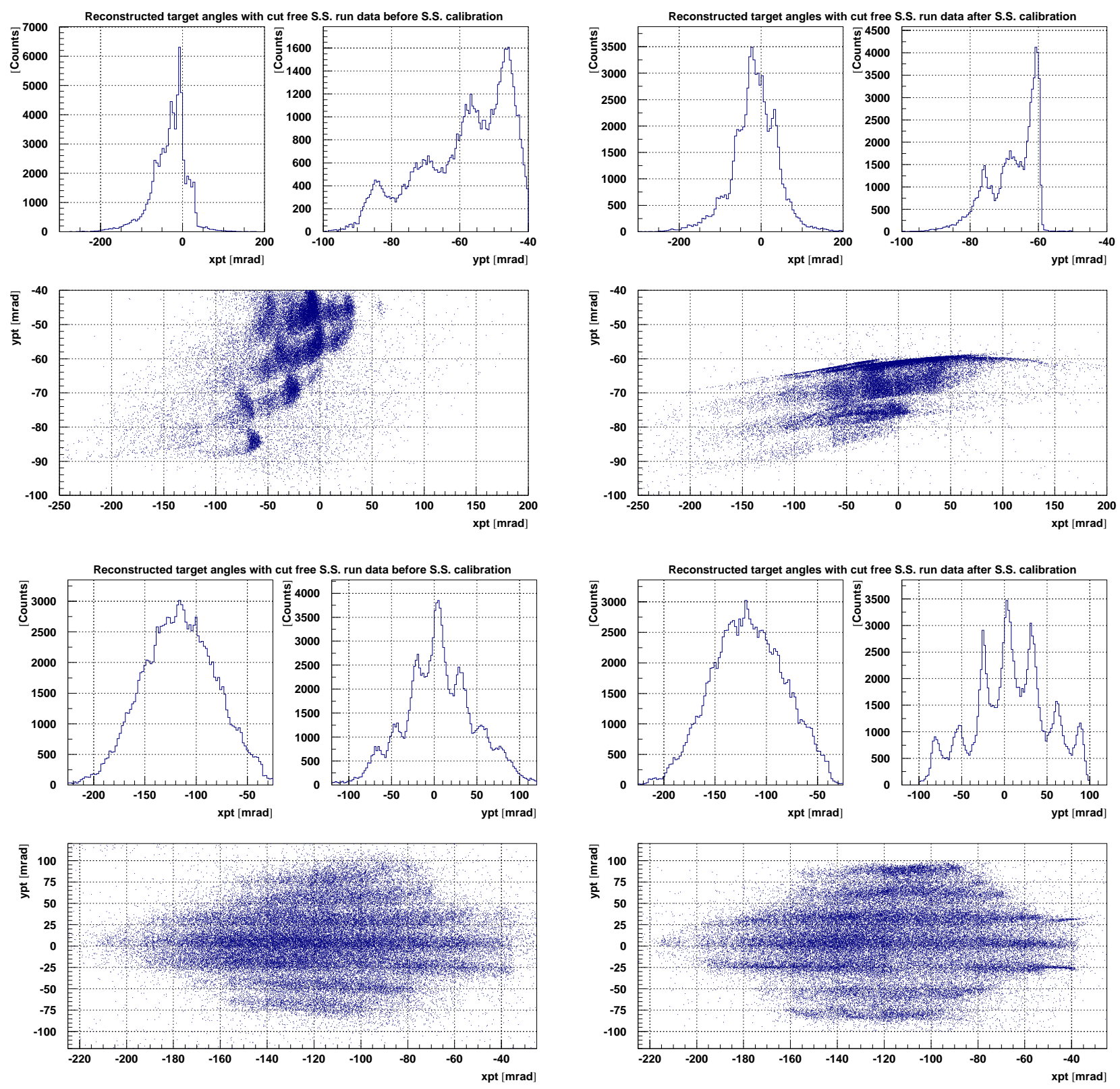

Figure 4.19: Comparison of the Enge (upper) and HKS (lower) reconstructed emission angle with (right)/without (left) S.S. calibration.

\subsubsection{Momentum calibration}

A flow chart for the momentum calibration for both the Enge and HKS is shown in Fig. 4.20. The target quantities $\left(x_{t}^{\prime}, y_{t}^{\prime}, d p\right)$ for $\mathrm{CH}_{2}$ and one part of ${ }^{12} \mathrm{C}$ target run data sets have to be calculated by applying the optimized emission angle reconstruction matrices.

At this point, missing mass for well known mass; $\Lambda, \Sigma^{0}$ and ${ }_{\Lambda}^{12} \mathrm{~B}$ ground state can be calculated with the reconstructed 6 variables, i.e. the combination of the emission angles and momentum 
$\left(e x_{t}^{\prime}, e y_{t}^{\prime}, e d p\right)$ and $\left(h x_{t}^{\prime}, h y_{t}^{\prime}, h d p\right)$ for the Enge and HKS, respectively. Fitting for the reconstructed mass spectra for the above three known masses with a Gaussian, events for momentum calibration are selected with $0.5 \sigma \sim 2 \sigma$ width of gates. Then minimizing $\chi^{2}$ as defined by Eqn. 4.8 , the particle momentum is calibrated.

$$
\chi^{2}=\sum_{i} w_{i} \Delta M_{i}^{2}=\sum_{i}\left[w_{i}\left\{M_{i}\left(e x_{t}^{\prime}, e y_{t}^{\prime}, e d p, h x_{t}^{\prime}, h y_{t}^{\prime}, h d p\right)-M_{i}^{\mathrm{PDB}}\right\}^{2}\right]
$$

where the subscript $i$ represents $\Lambda, \Sigma^{0}$ and ${ }_{\Lambda}^{12} \mathrm{~B}$ g.s., $w_{i}$ are the relative weights for the known masses, $M_{i}$ is the reconstructed mass for particle $i$ and $M_{i}^{\mathrm{PDB}}$ is the mass published in the Particle Data Book for particle $i$.

$$
w_{i}=\frac{c_{i} \exp \left(-\beta_{i} \Delta M_{i}^{2}\right)}{\exp \left(-\beta_{i} \lambda_{i}\right)+\exp \left(-\beta_{i} \Delta M_{i}^{2}\right)}
$$

where $\beta_{i}$ and $\lambda_{i}$ are free parameters defined as $\sim 0.5 \sigma^{2}$ and $\sim(2.5 \sigma)^{2}$ with an expected mass resolution $\sigma$ for particle $i$, respectively. $c_{i}$ is a constant.

Eventually, solving a non-linear least square equation which consists of both optimized emission angles and momentum $\left(x_{t}^{\prime}, y_{t}^{\prime}, d p\right)$ and focal plane quantities $\left(x_{f}, x_{f}^{\prime}, y_{f}, y_{f}^{\prime}\right)$, the momentum reconstruction matrices can be optimized for each spectrometer system.

\section{ESPS/HKS Momentum Calibration Procedure}

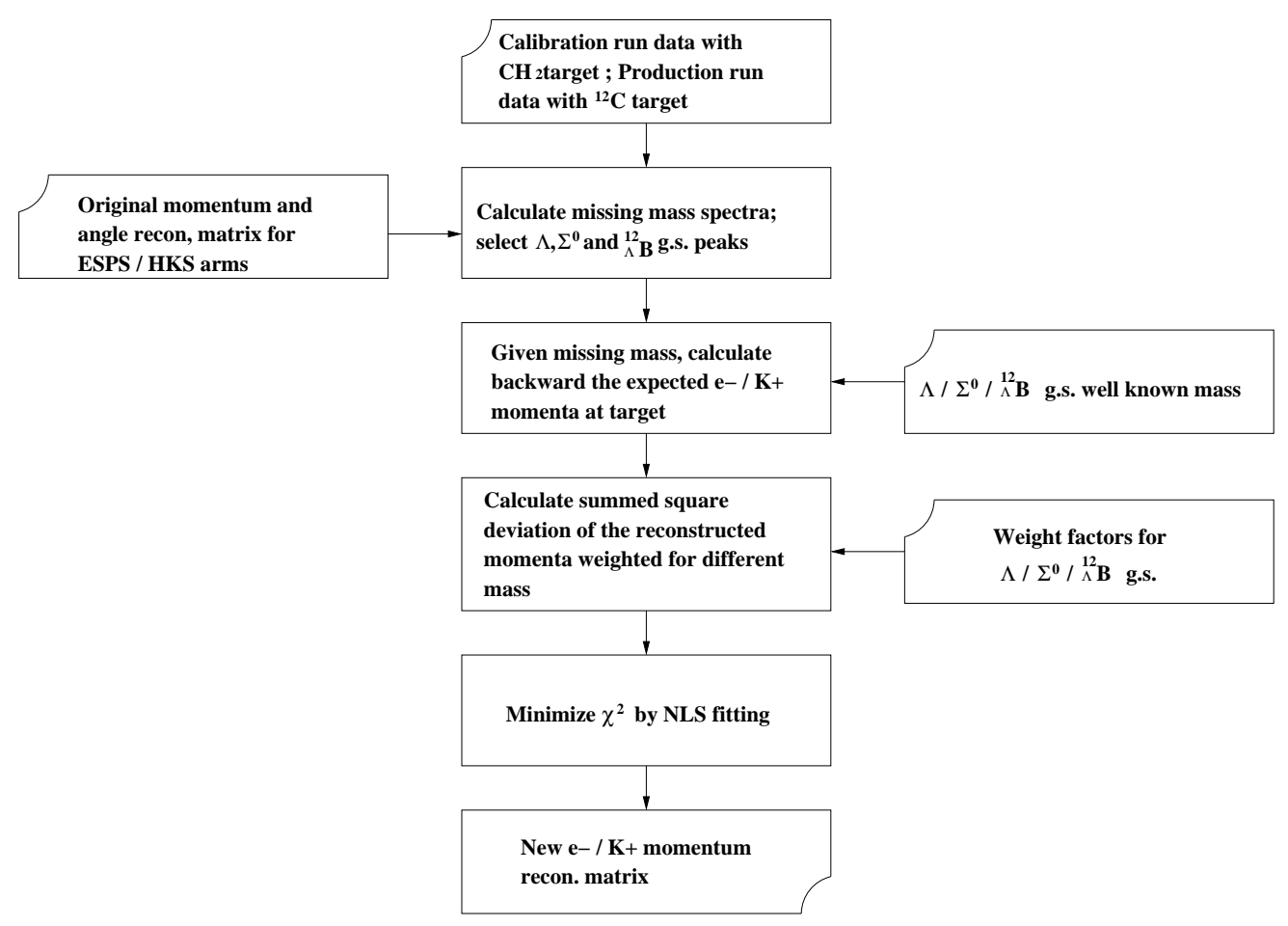

Figure 4.20: The flow chart for the momentum calibration procedure.

\subsection{Systematic errors of mass scale}

The missing mass spectra of the $\mathrm{p}\left(\mathrm{e}, \mathrm{e}^{\prime} \mathrm{K}^{+}\right) \Lambda / \Sigma^{0}$ reactions are shown in Fig. 4.21.

Hatched region below $\Lambda$ and $\Sigma^{0}$ in Fig. 4.21 represents background events associated with 
accidental coincidence and quasi-free production of hyperons on ${ }^{12} \mathrm{C}$ and $\mathrm{CH}_{2}$.

The proton mass is used as the target mass $\left(M_{A}\right)$ in the calculation of $M_{X}$ as described in Eqn. 4.5 (here, the subscript "HY" is $X$ ).

The $\Lambda$ and $\Sigma^{0}$ peaks are fitted by functions which consist of pseudo-Voigtian, Gaussian and 2nd order of polynomial equations as follows:

for $\Lambda$,

$$
\begin{aligned}
y & =p_{1}\left[p_{2} \frac{2}{\pi} \frac{p_{3}}{4\left(x-p_{4}\right)^{2}+p_{3}^{2}}+\left(1-p_{2}\right) \frac{\sqrt{4 \ln 2}}{\sqrt{\pi} p_{3}} \exp \left\{-\frac{4 \ln 2}{p_{3}{ }^{2}}\left(x-p_{4}\right)^{2}\right\}\right] \\
& +p_{5} \exp \left\{-\frac{\left(x-p_{4}\right)^{2}}{2 p_{3}{ }^{2}}\right\} \\
& +p_{6} x^{2}+p_{7} x+p_{8}
\end{aligned}
$$

and for $\Sigma^{0}$,

$$
\begin{aligned}
y & =p_{1}\left[p_{2} \frac{2}{\pi} \frac{p_{3}}{4\left(x-p_{4}\right)^{2}+p_{3}^{2}}+\left(1-p_{2}\right) \frac{\sqrt{4 \ln 2}}{\sqrt{\pi} p_{3}} \exp \left\{-\frac{4 \ln 2}{p_{3}{ }^{2}}\left(x-p_{4}\right)^{2}\right\}\right] \\
& \times p_{5} \exp \left\{-\frac{\left(x-p_{4}\right)^{2}}{2 p_{3}{ }^{2}}\right\} \\
& +p_{6} x^{2}+p_{7} x+p_{8}
\end{aligned}
$$

where $x=M_{X}-M_{\Lambda}$ and $y$ is count, respectively.

Fitting results by the above function are also shown in the same figure and Table 4.1.

In the elementally process, kinematic broadening dominates the resolution, and it decreases with the target mass number.

The systematic errors in the mass scales are determined by using the fitting errors of the peaks centered on $\Lambda$ and $\Sigma^{0}$. From Table 4.1, the systematic error is estimated to be $\sqrt{0.05^{2}+0.07^{2}} \simeq$ $0.1 \mathrm{MeV}$.

Table 4.1: Fitting results of $\Lambda$ and $\Sigma^{0}$ in the missing mass spectra.

\begin{tabular}{c|c|c}
\hline & $\Lambda$ & $\Sigma^{0}$ \\
\hline \hline$M_{X}-M_{\Lambda / \Sigma^{0}}\left[\mathrm{MeV} / c^{2}\right]$ & $0.02 \pm 0.05$ & $0.05 \pm 0.07$ \\
$\mathrm{FWHM}\left[\mathrm{MeV} / c^{2}\right]$ & $3.12 \pm 0.32$ & $2.65 \pm 0.33$ \\
\hline
\end{tabular}

\subsection{Cross section}

The cross section of the $\left(\gamma^{*}, \mathrm{~K}^{+}\right)$reaction is calculated from the experimental yields divided by the experimental efficiency as;

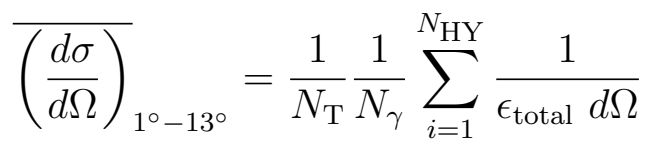

where $N_{\mathrm{T}}$ is the number of target, $N_{\gamma}$ the number of virtual photons, $d \Omega$ the solid angle acceptance of HKS, $N_{\mathrm{HY}}$ the yields of $\Lambda, \Sigma^{0}$ or a hypernuclear state, and $\epsilon_{\text {total }}$ efficiency. $\epsilon_{\text {total }}$ is defined by

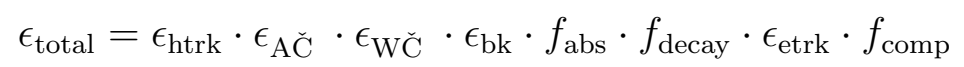




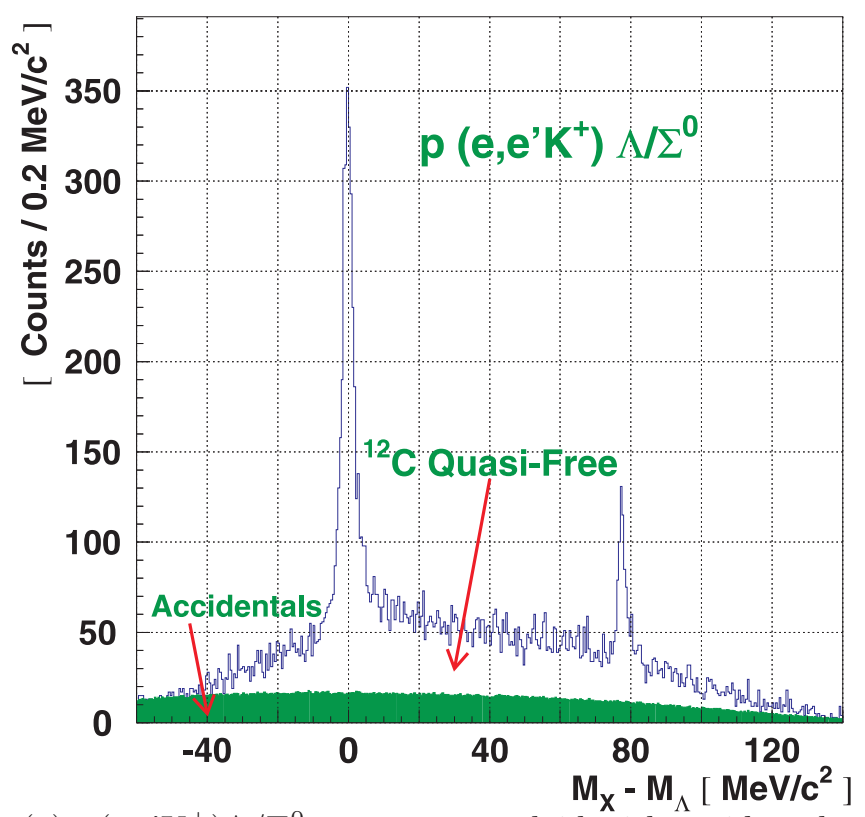

(a) $\mathrm{p}\left(\mathrm{e}, \mathrm{e}^{\prime} \mathrm{K}^{+}\right) \Lambda / \Sigma^{0}$ spectrum overlaid with accidentals

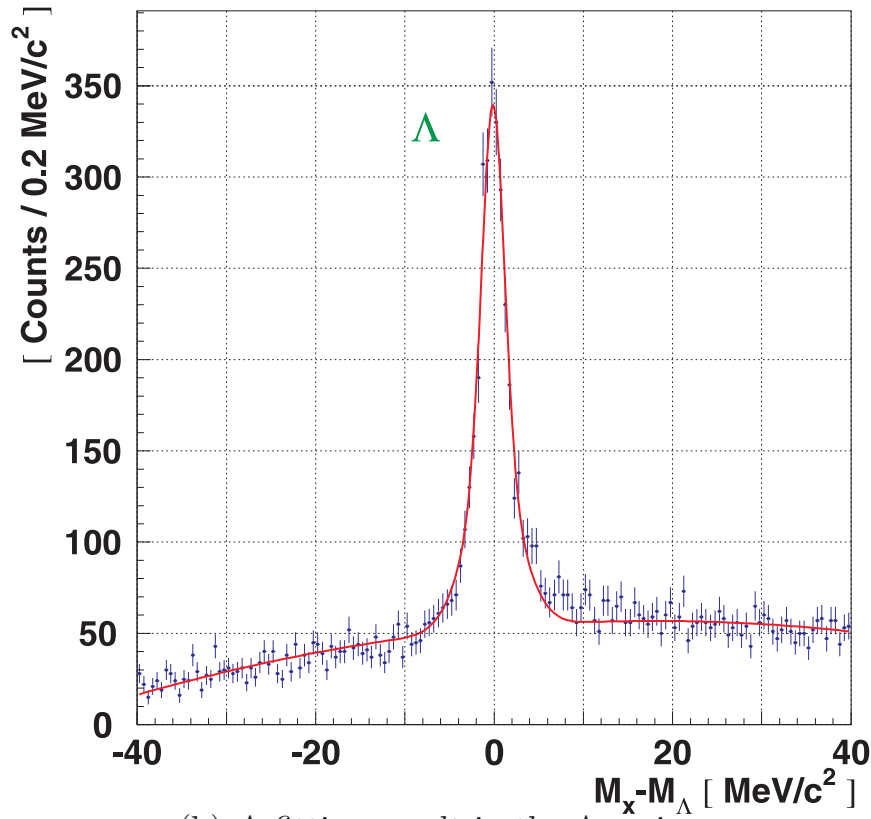

(b) A fitting result in the $\Lambda$ region

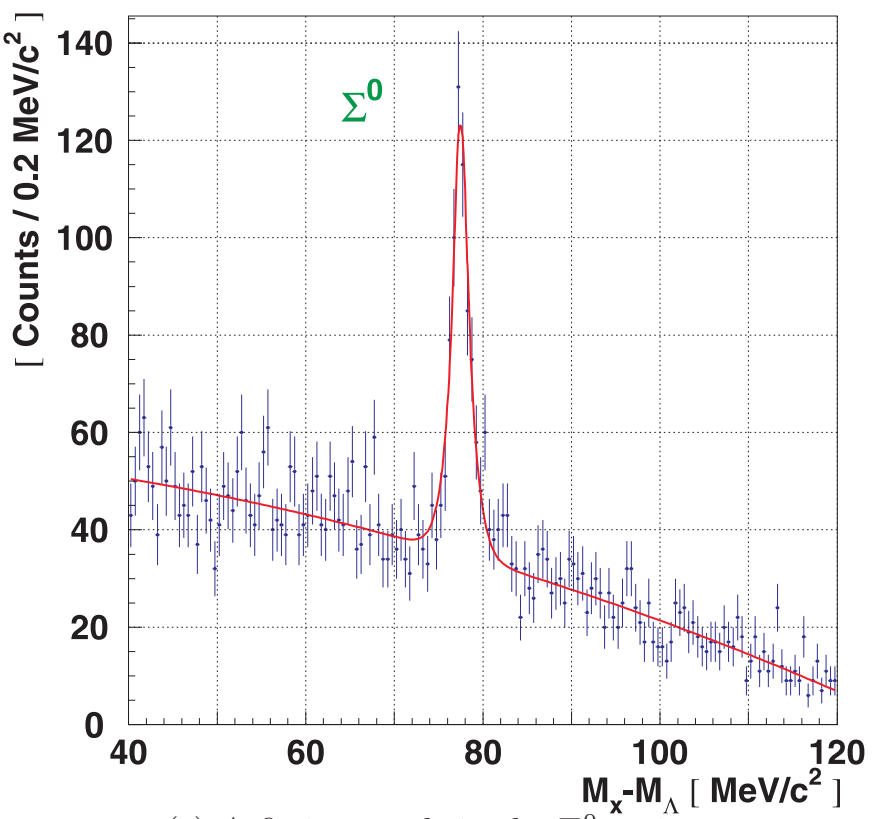

(c) A fitting result in the $\Sigma^{0}$ region

Figure 4.21: Missing mass spectra $\left(M_{X}-M_{\Lambda}\right)$ of $\mathrm{p}\left(\mathrm{e}, \mathrm{e}^{\prime} \mathrm{K}^{+}\right) \Lambda / \Sigma^{0}$ for $\mathrm{CH}_{2}$ runs. Fitted lines by the 2nd order of polynomial and a pseud-Voigt function and a Lorentzian are also described in the lower two figures. The error is statistical only. 
where the symbols are defined in Table 4.2.

Table 4.2: List of the experimental efficiency factors and typical (Data Set ID. \#6) values.

\begin{tabular}{|c|c|c|}
\hline $\begin{array}{c}\text { Factors and } \\
\text { efficiencies }\end{array}$ & Meanings & $\begin{array}{l}\text { Typical value } \\
\text { and error }\end{array}$ \\
\hline$\epsilon_{\mathrm{htrk}}$ & HKS tracking efficiency & $0.961 \pm 0.006$ \\
\hline$\epsilon_{\mathrm{AC}}$ & AČ cut efficiency & $0.957 \pm 0.010$ \\
\hline$\epsilon_{\mathrm{WČ} 1}$ & WČ1 cut efficiency & $0.954 \pm 0.009$ \\
\hline$\epsilon_{\mathrm{WC} 2}$ & WČ2 cut efficiency & $0.950 \pm 0.009$ \\
\hline$\epsilon_{\mathrm{bk}}$ & $\beta_{\mathrm{TOF}^{-}} \beta_{\mathrm{K}^{+}}$cut efficiency & $0.980 \pm 0.013$ \\
\hline$f_{\text {abs }}$ & Kaon absorption factor & $0.818 \pm 0.005$ \\
\hline$f_{\text {decay }}$ & Kaon decay factor & $0.345 \pm 0.001$ \\
\hline$\epsilon_{\text {etrk }}$ & Enge tracking efficiency & $0.881 \pm 0.002$ \\
\hline$f_{\text {comp }}$ & Computer live time factor & $0.970 \pm 0.002$ \\
\hline Total & & $0.197 \pm 0.007$ \\
\hline
\end{tabular}

\subsubsection{Acceptance}

\section{Momentum acceptance}

The correction of the reconstructed emission angles at the target point $(\theta, \phi$ with the spherical coordinates) for electron arm and kaon arm are shown in Fig. 4.22. From Fig. 4.22, the central zenith angles for HKS and Enge are deduced as $\phi_{e^{\prime}}=0.075 \mathrm{rad}$ and $\phi_{\mathrm{K}^{+}}=0.12 \mathrm{rad}$, respectively.
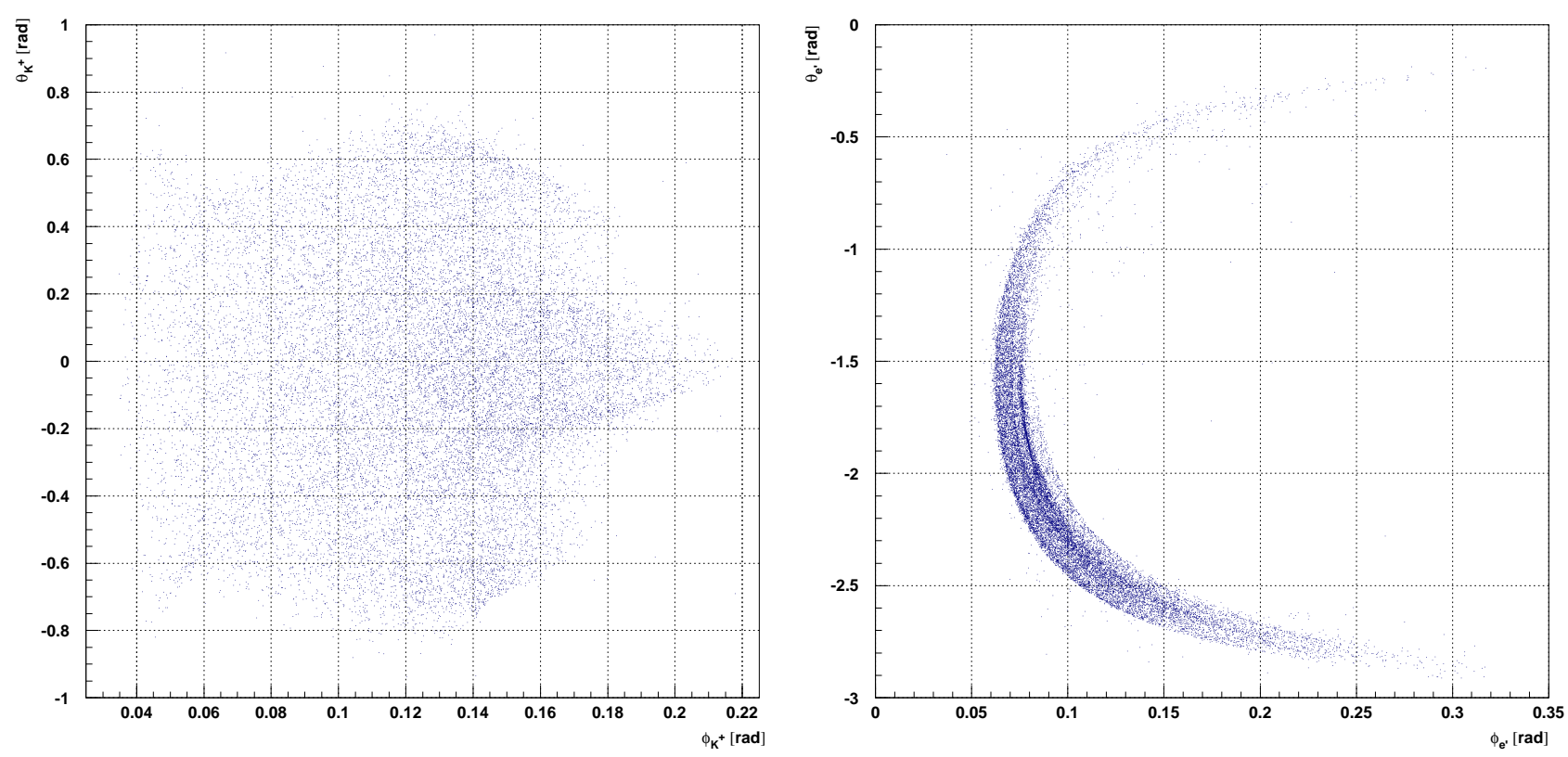

Figure 4.22: Reconstructed HKS and Enge emission angles at the target point.

The momentum correlation between electron and kaon arm for $\Lambda, \Sigma^{0}$ and ${ }_{\Lambda}^{12} \mathrm{~B}$ ground state pro- 
duction can be calculated as Fig. 4.23. For this calculation, the following kinematic condition are assumed;

- Incident electron beam energy : $E_{\mathrm{e}}=1.851 \mathrm{GeV}$,

- Scattering angles at the target point : $\phi_{e^{\prime}}=0.075 \mathrm{rad}$ for the scattered electron, $\phi_{\mathrm{K}^{+}}=$ $0.12 \mathrm{rad}$ for the kaon.

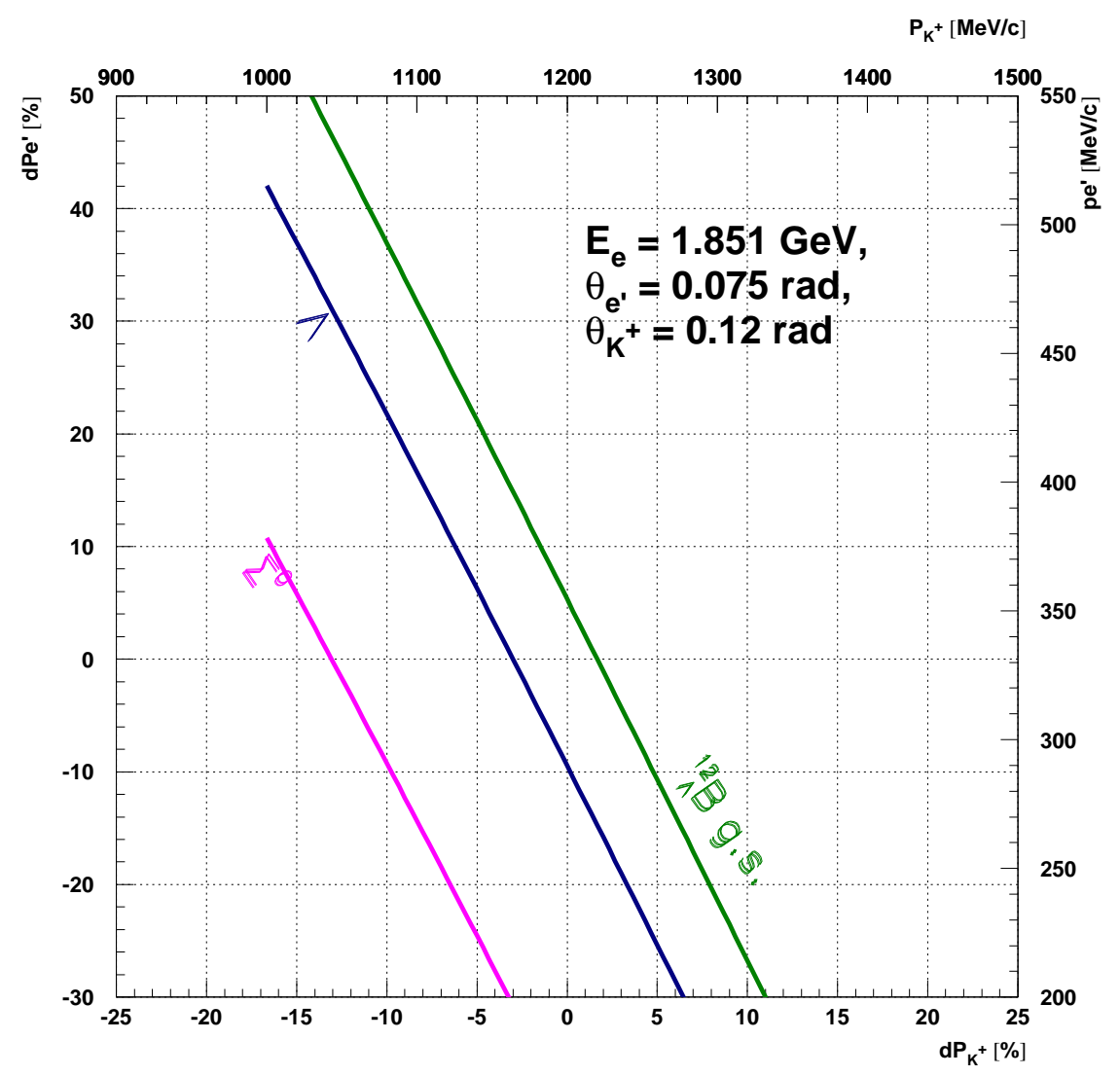

Figure 4.23: Calculated Enge and HKS momentum correlation for $\Lambda, \Sigma^{0}$ and ${ }_{\Lambda}^{12} \mathrm{~B}$ ground state production. $E_{\mathrm{e}}=1.851 \mathrm{GeV}$ of the incident electron beam energy, scattering angles at the target point; $\phi_{e^{\prime}}=0.075 \mathrm{rad}$ and $\phi_{\mathrm{K}^{+}}=0.12 \mathrm{rad}$ for electrons and kaons, respectively, are assumed.

On the other hand, the realistic Enge/HKS momentum acceptance has been deduced with the momentum reconstruction matrices (see Section 4.5.3) as $p_{e^{\prime}}=365.6 \mathrm{MeV} / c \pm 35.0 \%$ and $p_{\mathrm{K}^{+}}$ $=1200.0 \mathrm{MeV} / c \pm 12.5 \%$, respectively. Fig. 4.24 (upper), 4.24 (lower) show the reconstructed Enge/HKS momentum correlation for the $\mathrm{CH}_{2}$ and ${ }^{12} \mathrm{C}$ target data, respectively. The calculated correlations are also overlayed with solid lines on each figure. Result of the precise emission angle, momentum and mass scale correction, the experimental momentum correlations for all hyperons and hypernuclear production are in good agreement with the calculated ones.

\section{Enge/HKS solid angle acceptance}

The Enge and HKS solid angle acceptances were derived by RAYTRACE and GEANT calculation with following kinematic conditions;

Enge parameters : 

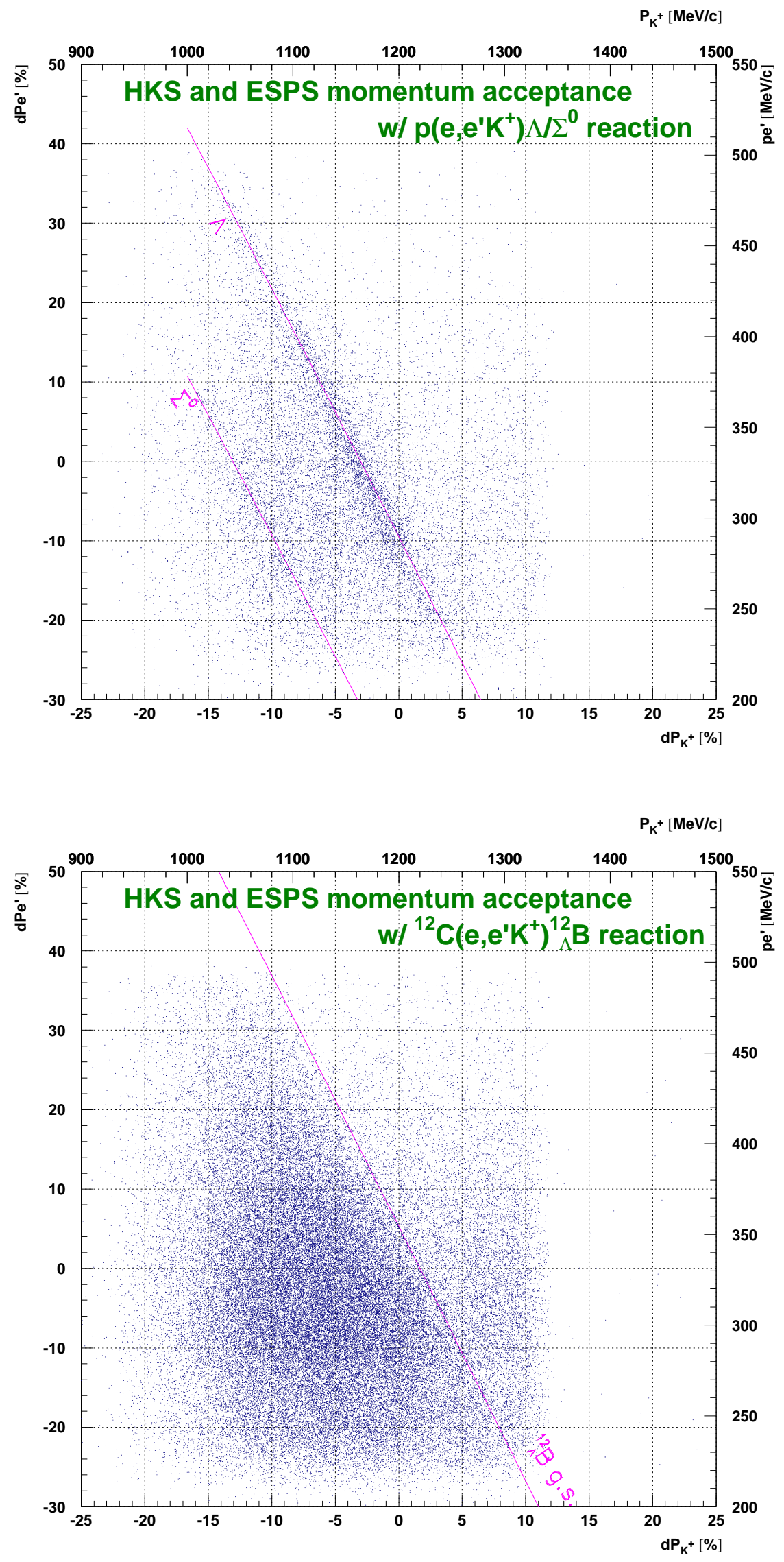

Figure 4.24: Reconstructed Enge and HKS momentum correlations for $\mathrm{CH}_{2}$ (upper) and ${ }^{12} \mathrm{C}($ lower $)$ target data. The calculation are also overlaid as solid lines. 
- $\Delta \theta: 0.05 \mathrm{rad}<\theta<0.31 \mathrm{rad}$

- $\Delta \phi: 0 \mathrm{rad}<\phi<2 \pi \mathrm{rad}$

- $\Delta p_{\mathrm{e}^{\prime}}: 188 \mathrm{MeV} / c<p_{e^{\prime}}<507 \mathrm{MeV} / c$

\section{HKS parameters :}

- $\Delta x_{t}^{\prime}:-270 \mathrm{mrad}<x_{t}^{\prime}<270 \mathrm{mrad}$

- $\Delta y_{t}^{\prime}:-270 \mathrm{mrad}<y_{t}^{\prime}<270 \mathrm{mrad}$

- $\Delta p_{\mathrm{K}^{+}}: 950 \mathrm{MeV} / c<p_{\mathrm{K}^{+}}<1450 \mathrm{MeV} / c$

The momentum dependent solid angle $S\left(p_{i}\right)\left(i=\mathrm{e}^{\prime}, \mathrm{K}^{+}\right)$for each spectrometer can be calculated as shown in Eqn. 4.14 and the results are represented in Fig. 4.25.

$$
S\left(p_{i}\right)=\Delta \Omega_{i} \frac{\Delta p_{i}}{p_{i}} \frac{\# \text { of accepted events }}{\# \text { of generated events }}
$$

In Eqn. 4.14, the subscript $i$ represents the Enge arm and the HKS arm. $\Omega_{i}$ represents the emission angular acceptance, $\Delta \theta \times \Delta \phi$ for the Enge, $\Delta x_{t}^{\prime} \times \Delta y_{t}^{\prime}$ for the HKS.
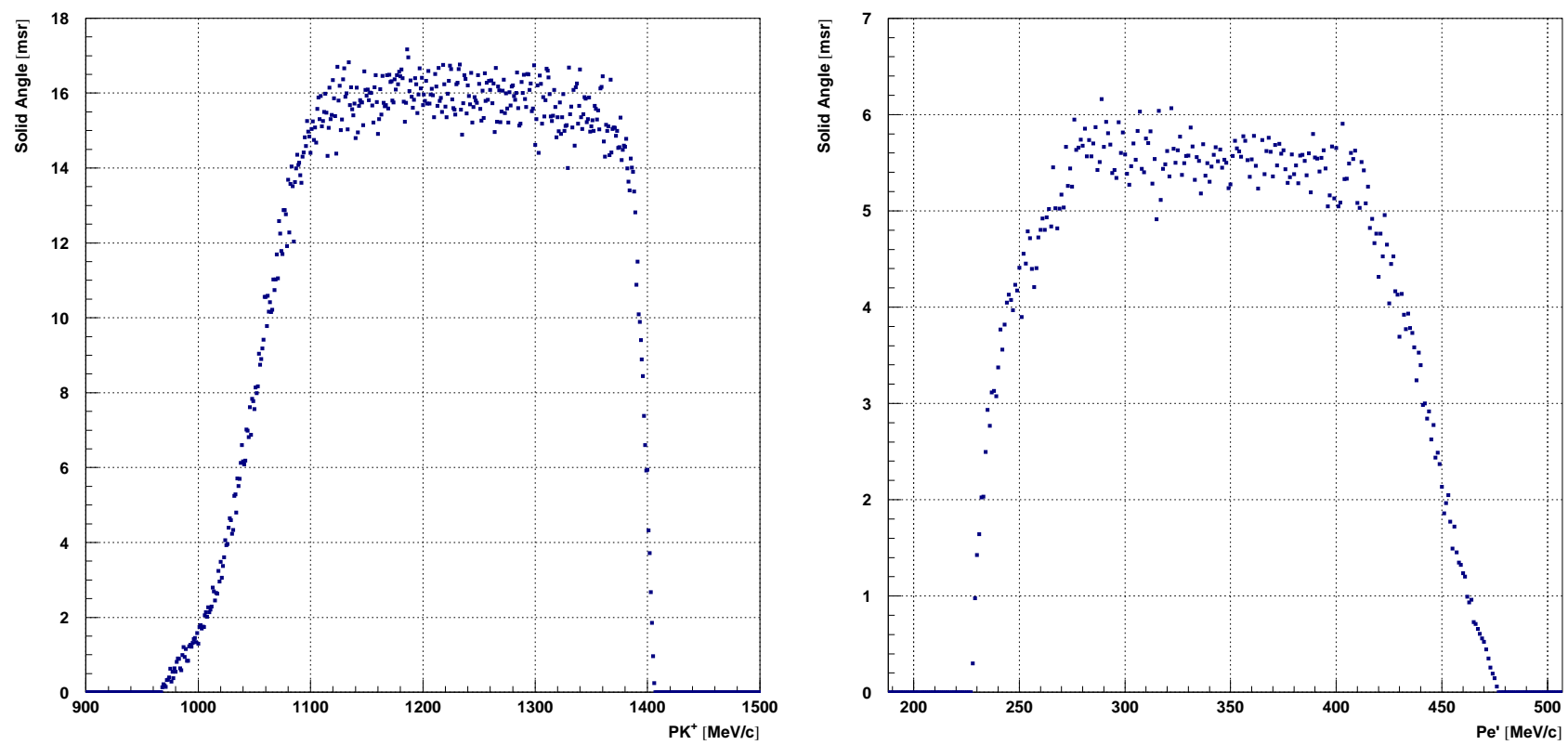

Figure 4.25: Momentum dependence of the HKS (left) and Enge (right) solid angle.

The statistical error for each solid angle calculation is estimated as $1 \%$.

\subsubsection{Number of virtual photons}

As discussed in subsection 2.2.1, the number of virtual photons $\left(N_{\gamma}\right)$ produced at the target point can be deduced by integrating the virtual photon flux; $\Gamma\left(\omega, \theta_{e^{\prime}}\right) \equiv \Gamma\left(E_{\text {beam }}=\right.$ $\left.1.851 \mathrm{GeV}, \omega, \theta_{e^{\prime}}\right)\left[/ \mathrm{MeV} /\right.$ electron] with the Enge solid angle; $S\left(p_{e^{\prime}}\right)$ as shown in Eqn. 4.15.

$$
N_{\gamma}=\frac{Q}{e} \iint \Gamma\left(\omega, \theta_{e^{\prime}}\right) S\left(p_{e^{\prime}}\right) d \omega d \Omega,
$$


where $Q$ is total charge, $e=1.60217733 \times 10^{-19}[\mathrm{C}]$ the elementary charge and $\omega$ the total energy of the virtual photon which covers $1339 \mathrm{MeV}<\omega<1604 \mathrm{MeV}$.

Note that, the e' scattering angle $\theta_{e^{\prime}}$ is the zenith angle.

Fig. 4.26 shows the momentum dependence of the virtual photon flux $\Gamma(\omega)$ in a unit of [/MeV/electron] integrated with the e' scattering angle $\theta_{e^{\prime}}$.

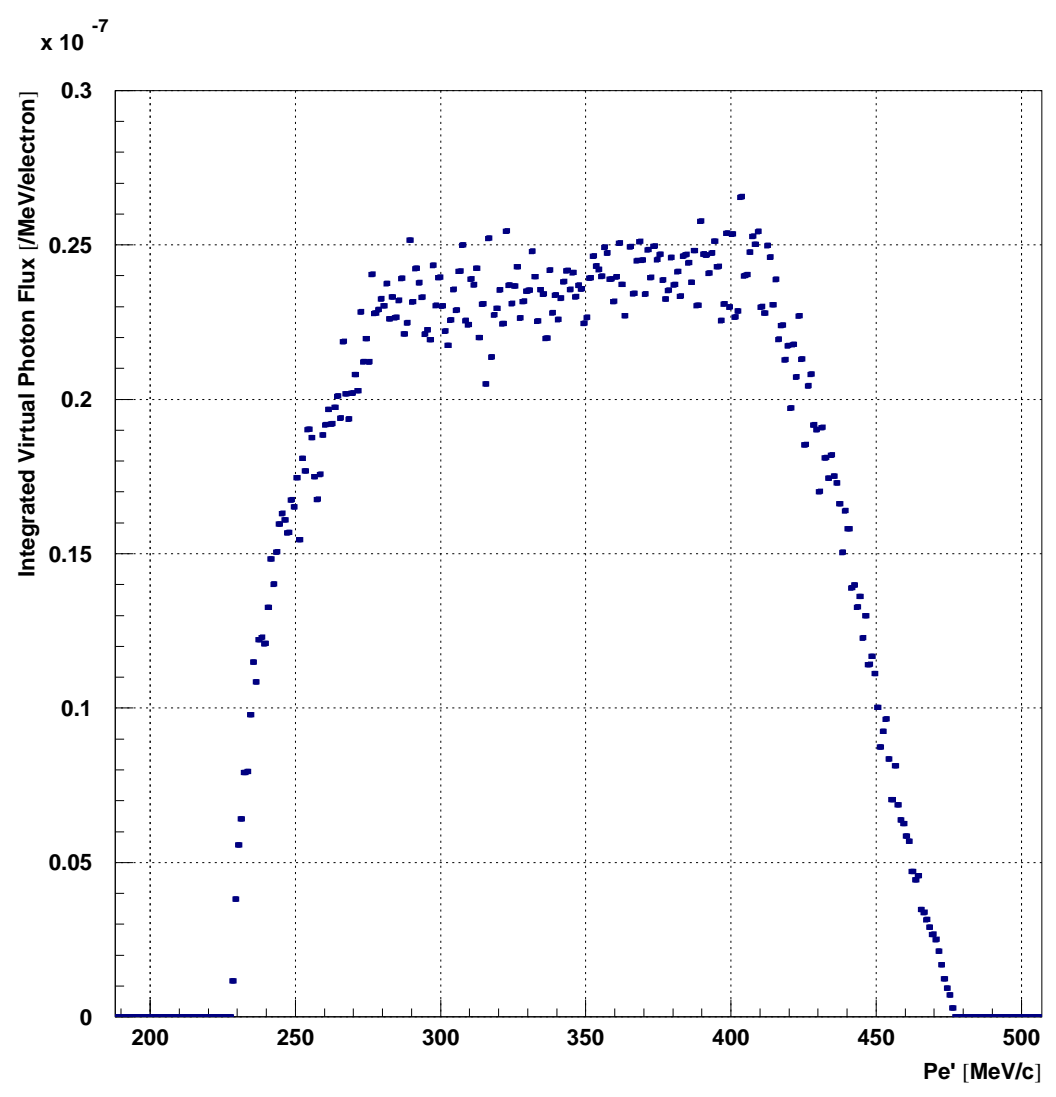

Figure 4.26: The virtual photon flux $\Gamma(\omega)$ integrated with e' scattering angle $\theta_{e^{\prime}}$.

Integrating the product of virtual photon flux $\Gamma(\omega)$ and the virtual photon energy $\omega$, the number of virtual photons per electron was estimated to be $4.805 \times 10^{-6}$ for the fixed electron beam energy of $1.851 \mathrm{GeV}$.

The systematic errors from the beam current measurement and magnet alignment accuracy are dominant. The beam current monitor was calibrated before the beam time. It showed a systematic error of less than $2 \%$. On the other hand, the accuracy of the magnet alignment was estimated to be $0.25 \mathrm{~mm}$. The systematic error due to misalignment was estimated to be $22 \%$. Compared to this, the systematic errors are negligible.

\subsubsection{Correction factors}

Since protons, kaons and pions overlap each other in the $\beta_{\text {TOF }}$ spectrum as shown in Fig. 4.27, the numbers of protons, kaons and pions are evaluated by fitting the $\beta_{\text {TOF }}$ spectrum for each data set listed in Table 2.13 for efficiency estimation. Table 4.3 shows a summary of the number of kaons evaluated by fitting $\beta_{\text {TOF }}$ spectra with single Gaussians. 


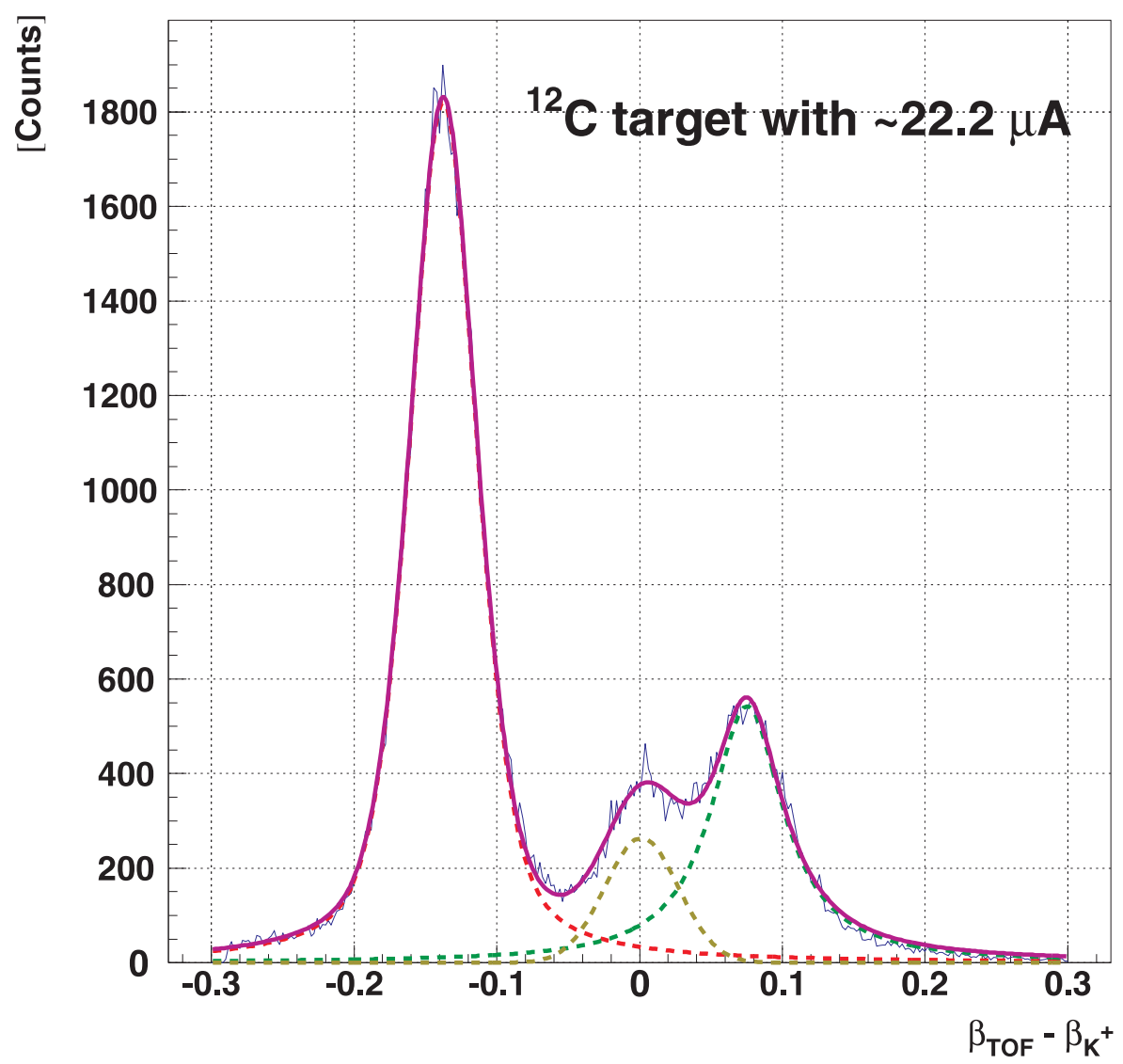

Figure 4.27: $\beta_{\text {TOF }}$ spectrum without any offline Čerenkov cut. 
Table 4.3: A summary of the number of kaons for each data set deduced by $\beta_{\mathrm{TOF}}-\beta_{\mathrm{K}^{+}}$fitting. The number of kaon is counted by integrating single Gaussian fit. The data sets of ${ }^{208} \mathrm{~Pb}$ (\# 22) are not included for physics analysis due to insufficient statistics.

\begin{tabular}{c|c|c|c|c|c}
\hline $\begin{array}{c}\text { Data set } \\
\text { No. }\end{array}$ & $\begin{array}{c}\text { Beam current } \\
\text { (Ave.) }[\mu \mathrm{A}]\end{array}$ & Target & Grouping & $\begin{array}{c}\text { Number of } \\
\text { kaons }\end{array}$ & $\begin{array}{c}\text { Beam charge } \\
{[\mathrm{mC}]}\end{array}$ \\
\hline \hline$\# 1$ & 2.3 & $\mathrm{CH}_{2}$ & OFF & 6839 & 247.2 \\
$\# 2$ & 16.1 & ${ }^{12} \mathrm{C}$ & OFF & 5289 & 618.5 \\
$\# 4$ & 19.7 & ${ }^{12} \mathrm{C}$ & OFF & 1331 & 249.8 \\
$\# 5$ & 2.3 & $\mathrm{CH}_{2}$ & ON & 9697 & 431.8 \\
$\# 6$ & 24.4 & ${ }^{12} \mathrm{C}$ & ON & 56115 & 6687.3 \\
$\# 7$ & 19.6 & ${ }^{9} \mathrm{Be}$ & ON & 31797 & 2367.5 \\
$\# 11$ & 21.3 & ${ }^{12} \mathrm{C}$ & ON & 14803 & 1129.7 \\
$\# 12$ & 21.6 & ${ }^{12} \mathrm{C}$ & ON & 5658 & 661.3 \\
$\# 13$ & 12.5 & ${ }^{28} \mathrm{Si}$ & ON & 16092 & 2531.7 \\
$\# 14$ & 22.4 & ${ }^{12} \mathrm{C}$ & ON & 50251 & 4633.1 \\
$\# 15$ & 12.7 & ${ }^{28} \mathrm{Si}$ & $\mathrm{ON}$ & 100449 & 11156.1 \\
$\# 16$ & 18.2 & ${ }^{9} \mathrm{Be}$ & $\mathrm{ON}$ & 26949 & 1316.3 \\
$\# 17$ & 12.4 & ${ }^{51} \mathrm{~V}$ & $\mathrm{ON}$ & 4465 & 699.6 \\
$\# 18$ & 10.6 & ${ }^{89} \mathrm{Y}$ & ON & 2310 & 516.0 \\
$\# 19$ & 24.9 & ${ }^{7} \mathrm{Li}$ & ON & 72923 & 3467.6 \\
$\# 20$ & 20.5 & ${ }^{6} \mathrm{Li}$ & ON & 47658 & 2273.3 \\
$\# 21$ & 26.5 & ${ }^{10} \mathrm{~B}$ & ON & 44865 & 3099.1 \\
\hline \hline
\end{tabular}

\section{Tracking efficiency}

The criteria of tracking efficiencies evaluation for the HKS arm and the Enge arm are basically the same.

They are calculated by the ratio of the number of tracks obtained by the drift chambers to that by the hodoscopes.

The procedures and results of the efficiency evaluation for each arm are presented below.

\section{(a) HKS tracking efficiency}

For the HKS arm, the tracking efficiency $\left(\epsilon_{\text {htrk }}\right)$ is evaluated with information from HDCs, HTOF1X, HTOF2X and 2 layers of WČs to select tracks associated with protons, since protons do not decay unlike the other particles in the HKS.

Rough tracking candidates are found by requiring 3 combinations of hit information as shown in Fig. 4.28 (a).

\section{(1) $\mathrm{HTOF} 1 \mathrm{X} \otimes \mathrm{HTOF} 2 \mathrm{X}$}

(2) $\mathrm{HTOF} 2 \mathrm{X} \otimes \mathrm{WČ} 1$

\section{(2) $\mathrm{HTOF} 2 \mathrm{X} \otimes \mathrm{WČ} 2$}

Then, events in which the tracks can be traced back to the center of HDCs are selected.

Finally for each selected event, HDC tracking is required. Eventually, the HKS tracking effi- 
ciency is evaluated as

$$
\mathrm{HKS} \text { tracking efficiency }=\frac{\mathrm{HTOF} 1 \mathrm{X} \otimes \mathrm{HTOF} 2 \mathrm{X} \otimes \mathrm{WC} \otimes \mathrm{HDC} \text { tracks }}{\mathrm{HTOF} 1 \mathrm{X} \otimes \mathrm{HTOF} 2 \mathrm{X} \otimes \mathrm{WC}} .
$$

The summary of the obtained HKS tracking efficiencies for entire run is represented in Fig.

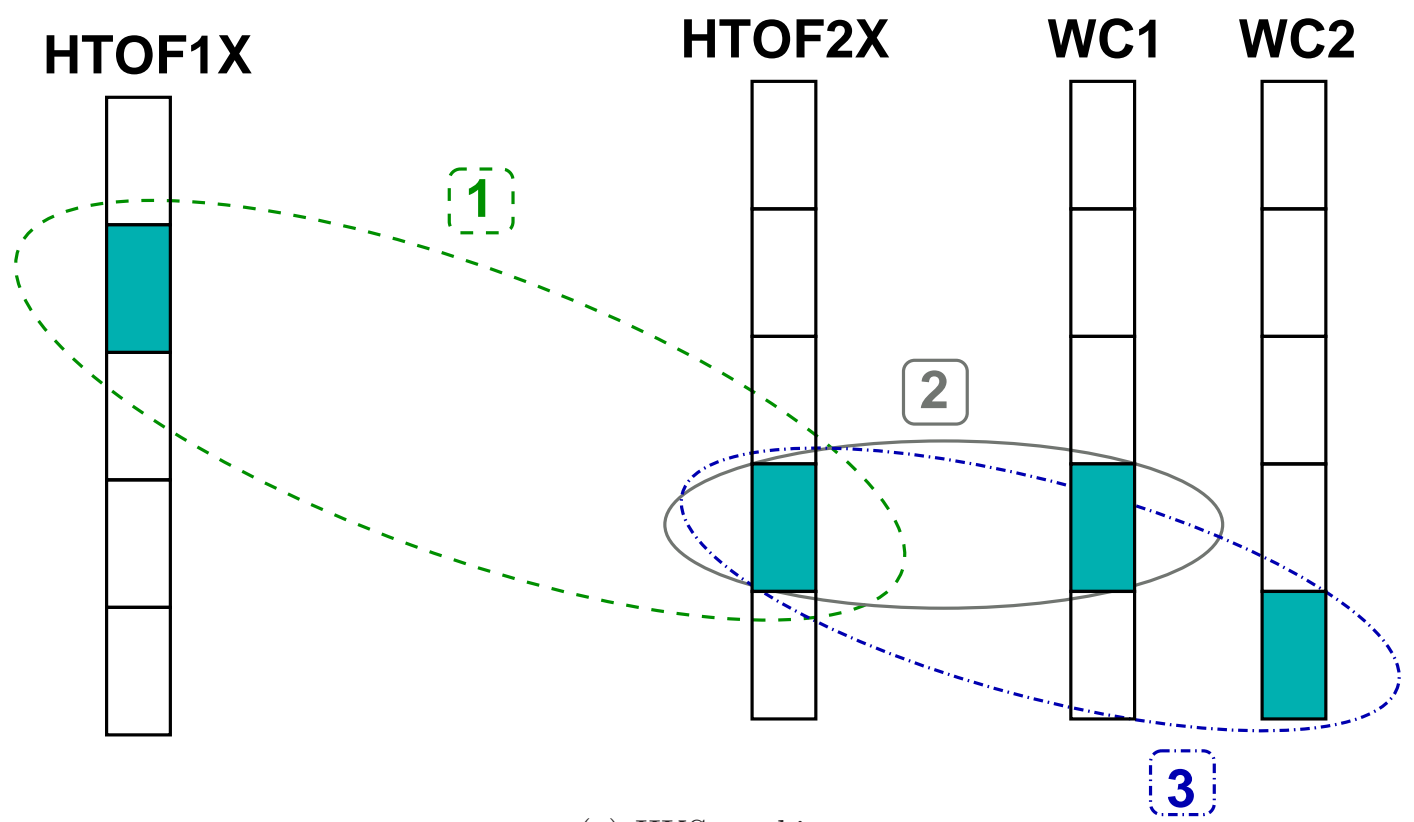

(a) HKS tracking

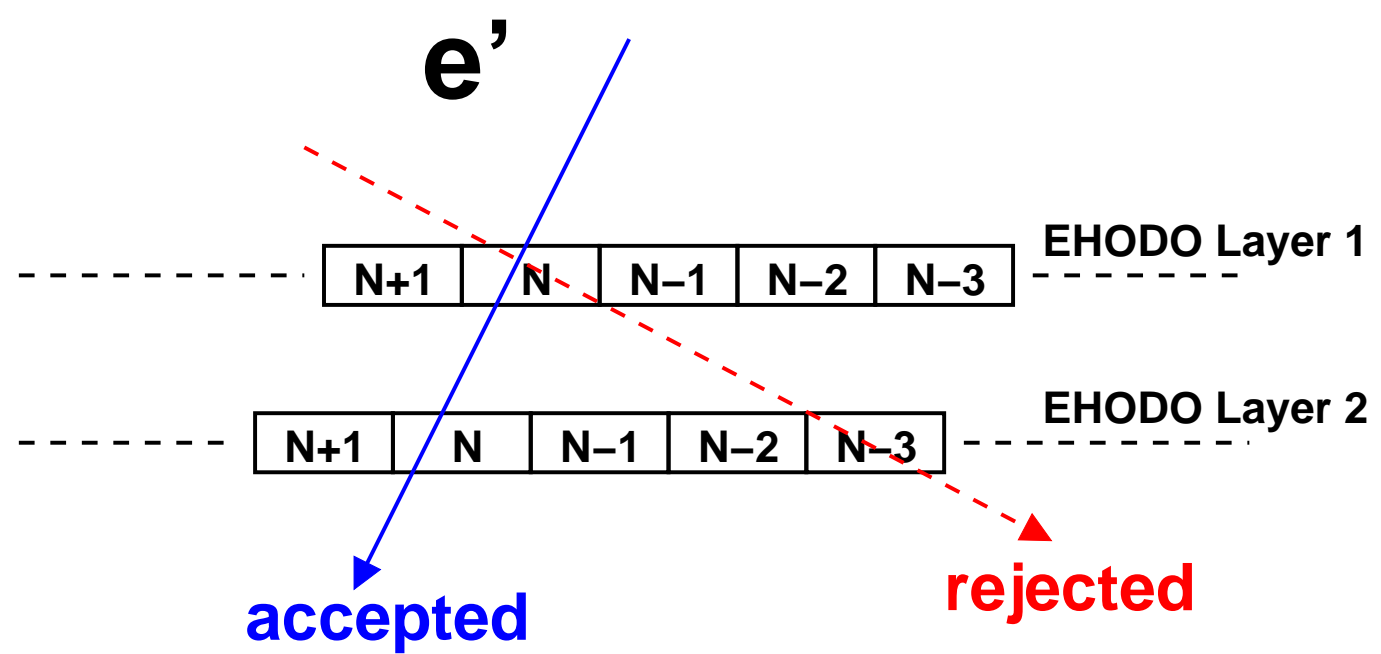

(b) Enge tracking

Figure 4.28: HKS / Enge tracking procedure.

4.29 (a). The weighted average value is $92.08 \pm 0.95 \%$.

(b) Enge tracking efficiency

The Enge tracking efficiency $\left(\epsilon_{\text {etrk }}\right)$ is extracted using track and hit information from the EDC and two layers of EHODOs. Similar to the case of the HKS, a brief tracking candidate is selected without EDC information as shown in Fig. 4.28 (b). The candidate is selected applying segment 
cut conditions :

$$
\mid \text { Counter ID (EHODO1) - Counter ID (EHODO2) } \mid \leq 2 \text {. }
$$

Finally, the Enge tracking efficiency is evaluated requiring the EDC track information as

$$
\text { Enge tracking efficiency }=\frac{\text { EHODO } 1 \otimes \text { EHODO2 } \otimes \text { EDC tracks }}{\text { EHODO1 } \otimes \text { EHODO2 }} .
$$

The obtained tracking efficiencies for entire run are summarized in Fig. 4.29 (b). The averaged efficiency is $91.57 \pm 0.26 \%$.

\section{AČ cut efficiency}

The A $\check{C}$ cut efficiency $\left(\epsilon_{\mathrm{AC}}\right)$ is evaluated by the ratio of the number of kaon events below a offline AČ threshold to that without an AČ cut. The offline AČ threshold is defined as sum of the number of photoelectrons detected on each layer :

$$
\text { Offline AČ threshold }=(\mathrm{AC} 1+\mathrm{AC} 2+\mathrm{AC} 3) .
$$

Since kaons and pions overlapped each other in the $\beta_{\text {TOF }}$ spectrum as shown in Fig. 4.27, kaon events are counted after subtracting pion events.

The number of pion events are evaluated by fitting the $\beta_{\text {TOF }}$ distribution with a Lorentzian.

The offline threshold dependence of the kaon survival ratio, i.e. AČ cut efficiency, for ${ }^{12} \mathrm{C}$ target runs with beam current of $22.2 \mu \mathrm{A}$ (Data Set No. 14) is shown in Fig. 4.30.

The AC cut efficiencies for the entire data set are summarized in Table 4.4, where the error is statistical only.

\section{WČ cut efficiency}

The WČ cut efficiency $\left(\epsilon_{\mathrm{WC}}\right)$ is evaluated by the ratio of the number of kaon events above a offline WČ threshold to that without a WČ cut. The offline WČ threshold is defined as sum of normalized number of photoelectrons detected on two layers :

$$
\text { Offline WČ threshold }=(\mathrm{WČ} 1+\mathrm{WČ} 2) \text {. }
$$

Similar to the case of the AČ, kaons and protons overlap each other in the $\beta_{\text {TOF }}$ spectrum as shown in Fig. 4.27, kaon events are counted after subtracting proton events.

The number of proton events is evaluated by fitting the $\beta_{\text {TOF }}$ distribution with a pseudo-Voigt function as described as follow :

$$
y=c_{1}\left[c_{2} \frac{2}{\pi} \frac{c_{3}}{4\left(x-c_{4}\right)^{2}+c_{3}^{2}}+\left(1-c_{2}\right) \frac{\sqrt{4 \ln 2}}{\sqrt{\pi} c_{3}} e^{-\frac{4 \ln 2}{c_{3}^{2}}\left(x-c_{4}\right)^{2}}\right] .
$$

Since the original summed number of photoelectrons in the WČ 1 and WČ2 monotonically decreased through out the entire data taking, probablly due to radiation damage, we define the normalized number of photoelectrons for each layer with the original one at the kaon peak. The offline threshold dependence of kaon survival ratio, i.e. WC cut efficiency, for ${ }^{12} \mathrm{C}$ target run with beam current of $22.2 \mu \mathrm{A}$ (Data Set No. 14) is shown in Fig. 4.31.

The WČ cut efficiencies for the entire data set are summarized in Table 4.5, where the errors are statistical only. 


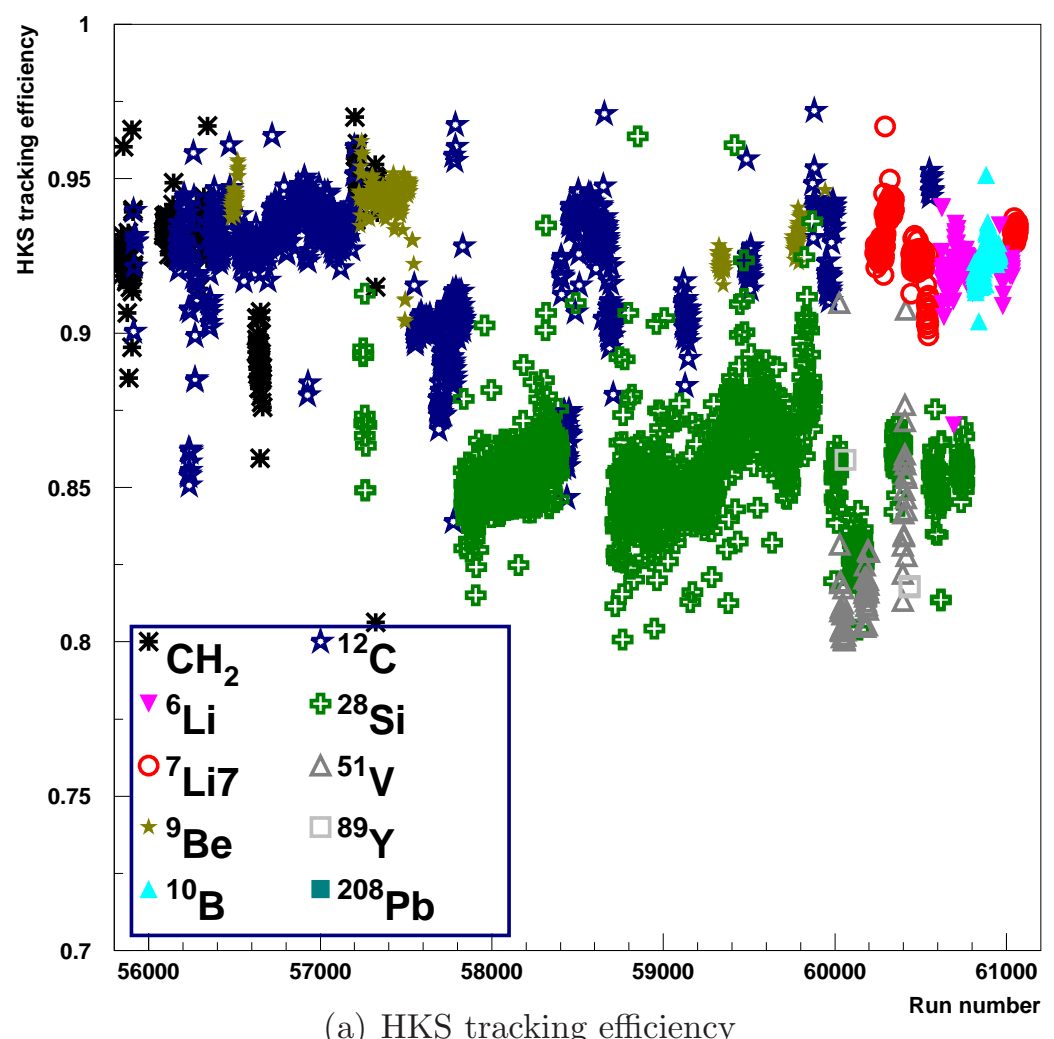

(a) HKS tracking efficiency

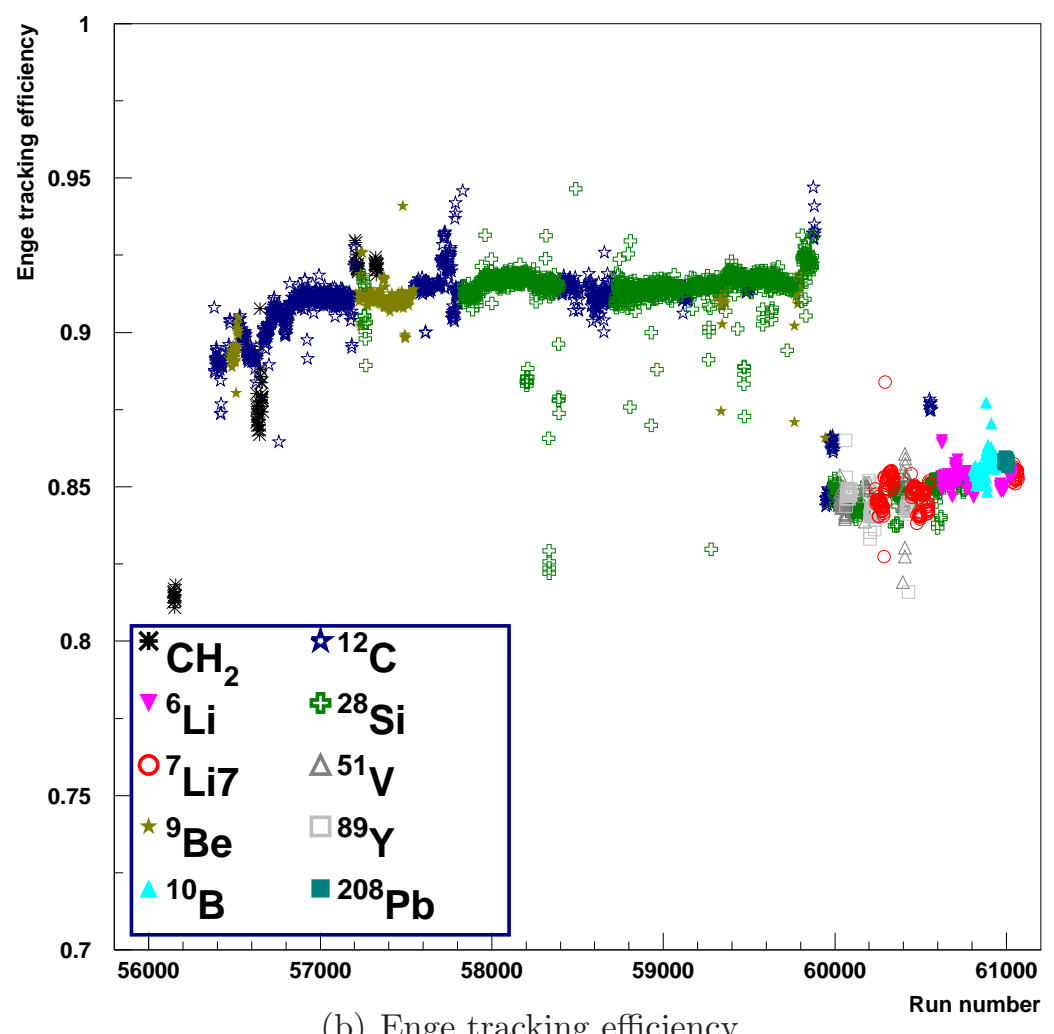

(b) Enge tracking efficiency

Figure 4.29: Run dependence of the HKS / Enge tracking efficiency. 


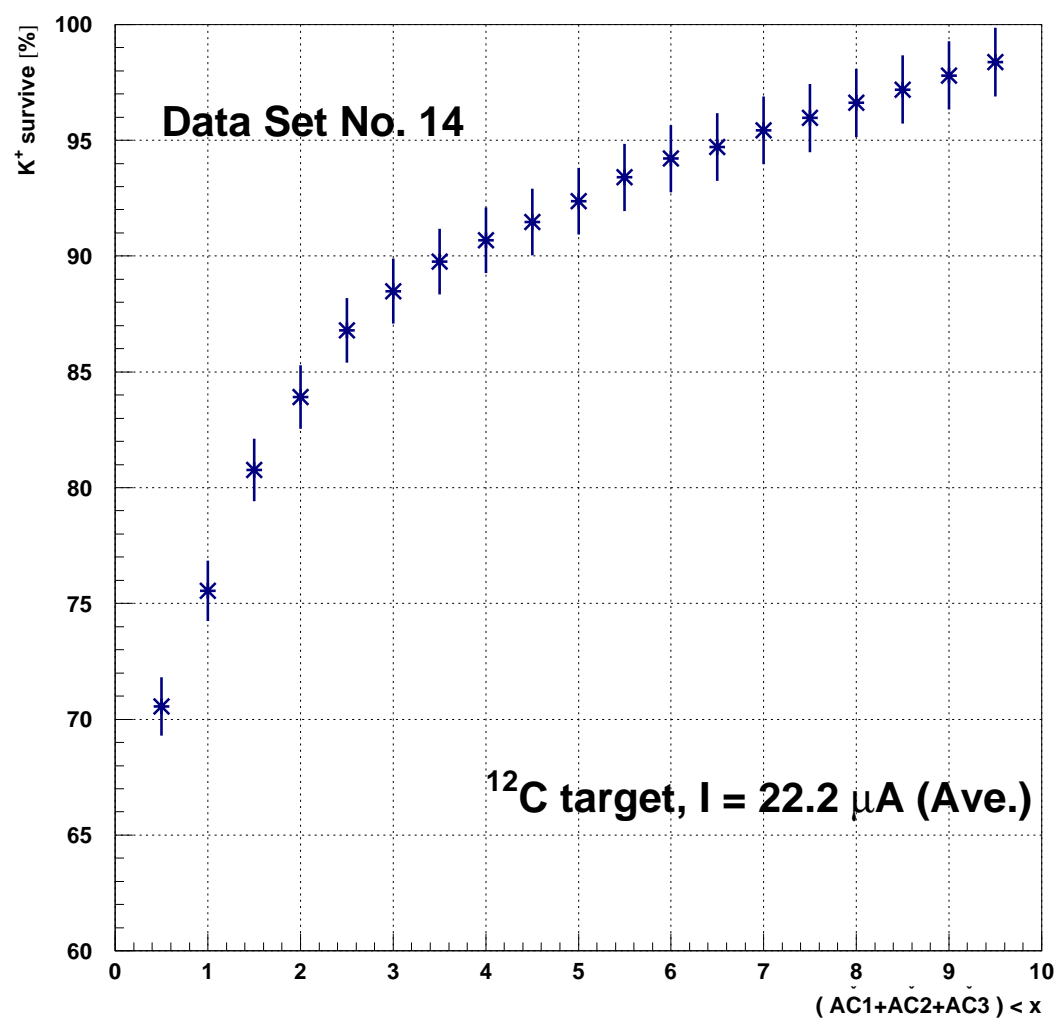

Figure 4.30: Correlation of kaon survival ratio and $\mathrm{AC}$ threshold in the case of ${ }^{12} \mathrm{C}$ target run with $22.2 \mu \mathrm{A}$ (Ave.) beam current.

Table 4.4: A summary of the AČ cut efficiencies for each data set. Errors are statistical only.

\begin{tabular}{c|c|c|c}
\hline $\begin{array}{c}\text { Data Set No. } \\
\text { No. }\end{array}$ & $\begin{array}{c}\text { Beam current } \\
\text { (Ave.) }[\mu \mathrm{A}]\end{array}$ & Target & $\begin{array}{c}\text { Efficiency } \\
{[\%]}\end{array}$ \\
\hline \hline$\# 1$ & 2.3 & $\mathrm{CH}_{2}$ & $90.69 \pm 1.15$ \\
$\# 2$ & 16.1 & ${ }^{12} \mathrm{C}$ & $90.11 \pm 1.31$ \\
$\# 4$ & 19.7 & ${ }^{12} \mathrm{C}$ & $95.56 \pm 2.67$ \\
$\# 5$ & 2.3 & $\mathrm{CH}_{2}$ & $95.51 \pm 0.99$ \\
$\# 6$ & 24.4 & ${ }^{12} \mathrm{C}$ & $95.90 \pm 1.11$ \\
$\# 7$ & 19.6 & ${ }^{9} \mathrm{Be}$ & $97.15 \pm 1.11$ \\
$\# 11$ & 21.3 & ${ }^{12} \mathrm{C}$ & $97.63 \pm 1.62$ \\
$\# 12$ & 21.6 & ${ }^{12} \mathrm{C}$ & $95.42 \pm 2.24$ \\
$\# 13$ & 12.5 & ${ }^{28} \mathrm{Si}$ & $95.64 \pm 2.21$ \\
$\# 14$ & 22.4 & ${ }^{12} \mathrm{C}$ & $99.90 \pm 1.19$ \\
$\# 15$ & 12.7 & ${ }^{28} \mathrm{Si}$ & $95.35 \pm 1.09$ \\
$\# 16$ & 18.2 & ${ }^{9} \mathrm{Be}$ & $95.37 \pm 1.04$ \\
$\# 17$ & 12.4 & ${ }^{51} \mathrm{~V}$ & $95.13 \pm 1.46$ \\
$\# 18$ & 10.6 & ${ }^{89} \mathrm{Y}$ & $95.28 \pm 2.03$ \\
$\# 19$ & 24.9 & ${ }^{7} \mathrm{Li}$ & $95.34 \pm 1.03$ \\
$\# 20$ & 20.5 & ${ }^{6} \mathrm{Li}$ & $95.50 \pm 0.90$ \\
$\# 21$ & 26.5 & ${ }^{10} \mathrm{~B}$ & $95.41 \pm 0.93$ \\
\hline \hline
\end{tabular}




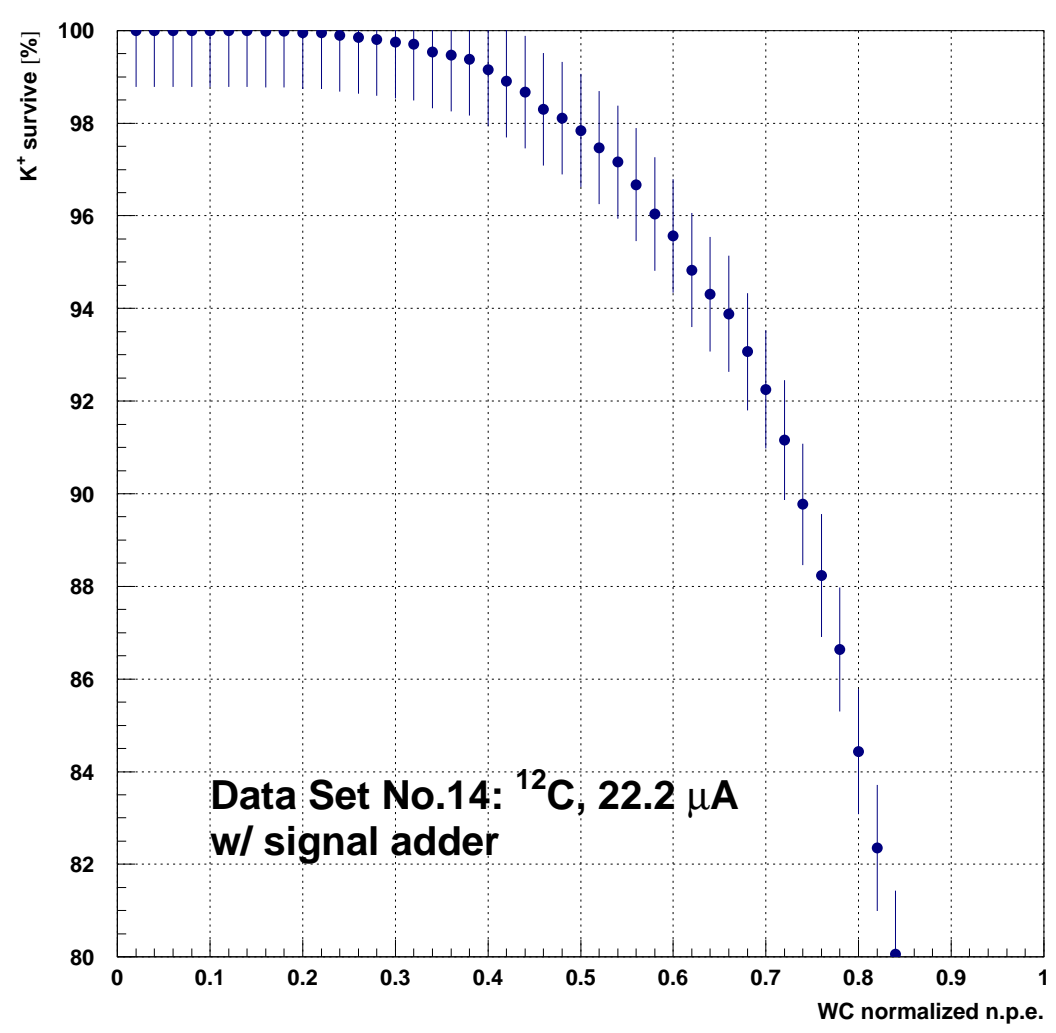

Figure 4.31: Correlation of kaon survival ratio and WČ threshold in the case of ${ }^{12} \mathrm{C}$ target run with $22.2 \mu \mathrm{A}$ (Ave.) beam current.

Table 4.5: A summary of the WČ cut efficiencies for each data set. Errors are statistical only.

\begin{tabular}{c|c|c|c|c}
\hline $\begin{array}{c}\text { Data Set No. } \\
\text { No. }\end{array}$ & $\begin{array}{c}\text { Beam current } \\
\text { (Ave.) }[\mu \mathrm{A}]\end{array}$ & $\begin{array}{c}\text { Target } \\
\text { Efficiency (WC1) } \\
{[\%]}\end{array}$ & $\begin{array}{c}\text { Efficiency (WC̈2) } \\
{[\%]}\end{array}$ \\
\hline \hline \# 1 & 2.3 & $\mathrm{CH}_{2}$ & $95.27 \pm 1.30$ & $95.10 \pm 1.30$ \\
$\# 2$ & 16.1 & ${ }^{12} \mathrm{C}$ & $95.41 \pm 1.45$ & $95.37 \pm 1.46$ \\
$\# 4$ & 19.7 & ${ }^{12} \mathrm{C}$ & $95.24 \pm 2.26$ & $95.76 \pm 2.28$ \\
$\# 5$ & 2.3 & ${ }^{2} \mathrm{H}_{2}$ & $95.39 \pm 0.84$ & $95.61 \pm 0.85$ \\
$\# 6$ & 24.4 & ${ }^{12} \mathrm{C}$ & $95.41 \pm 0.95$ & $95.27 \pm 0.95$ \\
$\# 7$ & 19.6 & ${ }^{9} \mathrm{Be}$ & $95.39 \pm 0.86$ & $95.12 \pm 0.86$ \\
$\# 11$ & 21.3 & ${ }^{12} \mathrm{C}$ & $95.45 \pm 1.57$ & $95.25 \pm 1.58$ \\
$\# 12$ & 21.6 & ${ }^{12} \mathrm{C}$ & $95.58 \pm 1.86$ & $95.43 \pm 1.92$ \\
$\# 13$ & 12.5 & ${ }^{28} \mathrm{Si}$ & $95.60 \pm 1.72$ & $95.60 \pm 1.77$ \\
$\# 14$ & 22.4 & ${ }^{12} \mathrm{C}$ & $95.42 \pm 1.21$ & $95.38 \pm 1.19$ \\
$\# 15$ & 12.7 & ${ }^{28} \mathrm{Si}$ & $95.38 \pm 0.89$ & $95.20 \pm 1.10$ \\
$\# 16$ & 18.2 & ${ }^{9} \mathrm{Be}$ & $95.66 \pm 1.07$ & $95.48 \pm 1.05$ \\
$\# 17$ & 12.4 & ${ }^{51} \mathrm{~V}$ & $95.59 \pm 1.42$ & $95.27 \pm 1.36$ \\
$\# 18$ & 10.6 & ${ }^{89} \mathrm{Y}$ & $95.44 \pm 1.90$ & $95.66 \pm 1.84$ \\
$\# 19$ & 24.9 & ${ }^{7} \mathrm{Li}$ & $95.57 \pm 1.09$ & $95.55 \pm 1.05$ \\
$\# 20$ & 20.5 & ${ }^{6} \mathrm{Li}$ & $95.61 \pm 0.91$ & $95.41 \pm 0.87$ \\
$\# 21$ & 26.5 & ${ }^{10} \mathrm{~B}$ & $95.58 \pm 0.98$ & $95.30 \pm 0.94$ \\
\hline \hline
\end{tabular}




\section{$\beta$ cut efficiency}

The original number of kaons for each data set is extracted by integrating a single Gaussian which is fit for kaon candidates on the $\beta_{\text {TOF }}$ spectrum without any offline Cerenkov cut as shown in Fig. 4.27, and the summary of the evaluated number of original kaons is listed in Table 4.3.

The $\beta$ cut efficiency $\left(\epsilon_{\mathrm{bk}}\right)$ is evaluated by the ratio of the number of kaon events with the $\beta$ cut $:\left|\beta_{\mathrm{TOF}}-\beta_{\mathrm{K}^{+}}\right|<x$ to that without $\beta$ cut.

A sample $\beta$ cut threshold (x) dependency of kaon survival ratio for ${ }^{12} \mathrm{C}$ run with a beam current of $22.2 \mu \mathrm{A}$ (Ave.) is represented in Fig. 4.32 .

A typical $\beta$ cut threshold is $\left|\beta_{\text {TOF }}-\beta_{\mathrm{K}^{+}}\right|<0.07$, and the $\beta$ cut efficiencies for the entire data set are summarized in Table 4.6.

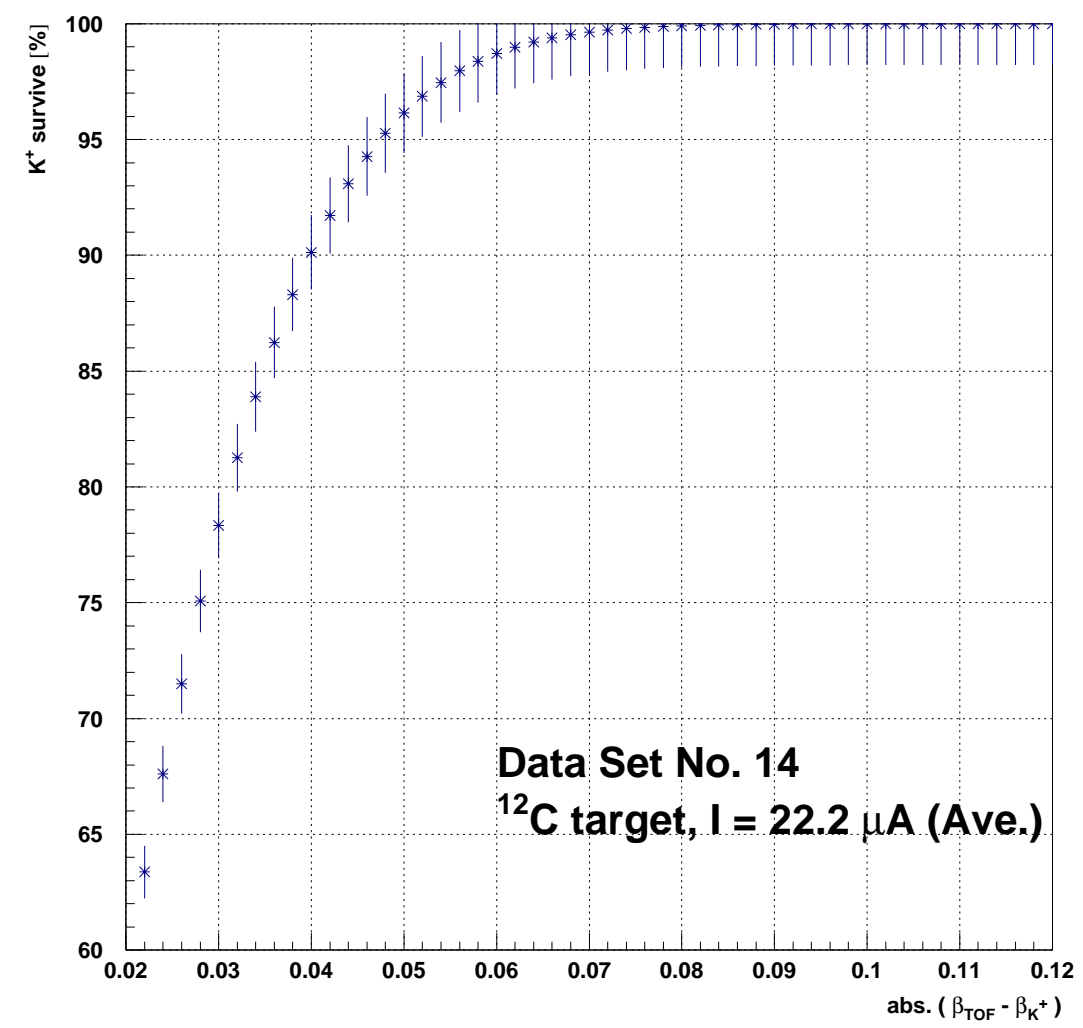

Figure 4.32: $\beta$ cut threshold dependency of $\mathrm{K}^{+}$survival ratio. The threshold is defined as the absolute value of the kaon velocity distribution; $a b s\left(\beta_{\mathrm{TOF}}-\beta_{\mathrm{K}^{+}}\right)$.

\section{Kaon absorption correction}

While a kaon is traveling from the target to the final layer of the water Cerrenkov counter, some fraction of the kaons are absorbed in materials including the target and counters, and thus may not be able to reach the final counter. Such events should be taken into account with a kaon absorption correction factor. Since this factor cannot be measured directly during the experiment, we calculated it with the properties of all materials which kaons passed through. A list of materials for the HKS arm with their thickness and densities is shown in Table 4.7. The kaon absorption reaction rate $\left(1-f_{\text {abs }}\right)$ can be written as:

$$
1-f_{\mathrm{abs}}=\sigma_{\text {inel }} \frac{N_{A} \rho t}{A},
$$


Table 4.6: A summary of the $\beta_{\mathrm{TOF}}-\beta_{\mathrm{K}^{+}}$cut efficiencies for each data set. Errors are statistical only. Statistical error for $\# 3,8,9$, and 10 are larger due to the inefficient statistics.

\begin{tabular}{c|c|c|c|c|c}
\hline $\begin{array}{c}\text { Data set } \\
\text { No. }\end{array}$ & $\begin{array}{c}\text { Beam current } \\
\text { (Ave. }[\mu \mathrm{A}]\end{array}$ & Target & Grouping & $\begin{array}{c}\text { Beam charge } \\
{[\mathrm{mC}]}\end{array}$ & Efficiency \\
\hline \hline$\# 1$ & 2.3 & $\mathrm{CH}_{2}$ & OFF & 247.7 & $96.53 \pm 1.68$ \\
$\# 2$ & 16.1 & ${ }^{12} \mathrm{C}$ & OFF & 626.6 & $96.58 \pm 1.87$ \\
$\# 4$ & 19.7 & ${ }^{12} \mathrm{C}$ & OFF & 255.2 & $92.30 \pm 3.38$ \\
$\# 5$ & 2.3 & $\mathrm{CH}_{2}$ & ON & 432.9 & $99.73 \pm 1.37$ \\
$\# 6$ & 24.4 & ${ }^{12} \mathrm{C}$ & ON & 6721.5 & $97.70 \pm 1.52$ \\
$\# 7$ & 19.6 & ${ }^{9} \mathrm{Be}$ & ON & 2400.5 & $97.63 \pm 1.53$ \\
$\# 11$ & 21.3 & ${ }^{12} \mathrm{C}$ & ON & 1133.0 & $94.75 \pm 2.19$ \\
$\# 12$ & 21.6 & ${ }^{12} \mathrm{C}$ & ON & 686.7 & $99.36 \pm 2.99$ \\
$\# 13$ & 12.5 & ${ }^{28} \mathrm{Si}$ & ON & 2804.0 & $99.09 \pm 2.94$ \\
$\# 14$ & 22.4 & ${ }^{12} \mathrm{C}$ & ON & 4662.5 & $99.42 \pm 1.74$ \\
$\# 15$ & 12.7 & ${ }^{28} \mathrm{Si}$ & ON & 11217.4 & $99.10 \pm 1.69$ \\
$\# 16$ & 18.2 & ${ }^{9} \mathrm{Be}$ & ON & 1321.2 & $99.37 \pm 1.56$ \\
$\# 17$ & 12.4 & ${ }^{51} \mathrm{~V}$ & ON & 700.3 & $98.66 \pm 2.30$ \\
$\# 18$ & 10.6 & ${ }^{89} \mathrm{Y}$ & ON & 520.7 & $97.65 \pm 3.07$ \\
$\# 19$ & 24.9 & ${ }^{7} \mathrm{Li}$ & ON & 3481.5 & $98.80 \pm 1.58$ \\
$\# 20$ & 20.5 & ${ }^{6} \mathrm{Li}$ & ON & 2276.6 & $98.94 \pm 1.32$ \\
$\# 21$ & 26.5 & ${ }^{10} \mathrm{~B}$ & ON & 3105.3 & $99.07 \pm 1.44$ \\
\hline \hline
\end{tabular}

where $\sigma_{\text {inel }}$ is the inelastic cross section, $A$ the mass number, $N_{A}=6.022 \times 10^{23}\left[\mathrm{~mol}^{-1}\right]$ is Avogadro's number and $\rho$ the density, $t$ the thickness of the material.

Considering all materials listed in Table $4.7, N_{A} \rho t / A$ amounts to $0.250 \sim 0.252[\% / \mathrm{mb}]$.

On the other hand, the total cross section and the elastic cross section around $1.05 \mathrm{GeV} / c$ $<p_{\mathrm{K}^{+}}<1.35 \mathrm{GeV} / c$ has been measured as $16.95 \mathrm{mb}<\sigma_{\text {tot }}<19.85 \mathrm{mb}$ [77-79], and $10.06<$ $\sigma_{\text {inel }}[\mathrm{mb}]<11.81[78,80,81]$, respectively. Thus the inelastic cross section can be expected to be less than $\sim 10 \mathrm{mb}$, and the kaon absorption rate was estimated as $\sim 2.5 \%$. The uncertainty associated with this correction is based only on a measurement accuracy of $\sim 1[\mathrm{~mm}]$ for the detector size, which corresponds to $0.01 \%$.

\section{Kaon decay factor}

The kaon is an unstable particle with a mean life time $\tau=(1.2385 \pm 0.0024) \times 10^{-8} \sec [82]$. Some of the kaons produced in the target decay before they reach the detectors. An efficiency factor associated with the $\mathrm{K}^{+}$decay $\left(f_{\text {decay }}\right)$ is calculated using a Monte Carlo simulation. As a reference, the principal decay channels of $\mathrm{K}^{+}$and their fraction are listed in Table 4.9.

We calculated the number of kaons which satisfied the following two requirements;

1: created a trigger passing through the two layers of the TOF counter (HTOF1X and HTOF2X),

2: reached to the second layer of water Cěrenkov counter (WČ2) and were recognized as kaons. 
Table 4.7: Properties of HKS materials. These parameters are used to calculate the kaon absorption factor. For $p[$ Torr $], t\left[{ }^{\circ} \mathrm{C}\right]$, air density $(\rho)$ is written as $\rho=101.325 /\{287.055 \times(273.15+t)\} \times p / 761.842$ $\left[\mathrm{g} / \mathrm{cm}^{3}\right]$.

\begin{tabular}{lccc}
\hline Material & $\mathrm{t}[\mathrm{cm}]$ & $\rho\left[\mathrm{g} / \mathrm{cm}^{3}\right]$ & Notes \\
\hline \hline Target & 0.5008 & 0.92 & \\
$\mathrm{CH}_{2}$ & 0.356 & 0.46 & \\
${ }^{6} \mathrm{Li}$ & 0.350 & 0.54 & \\
${ }^{7} \mathrm{Li}$ & 0.1007 & 1.848 & \\
${ }^{9} \mathrm{Be}$ & 0.041 & 2.16 & \\
${ }^{10} \mathrm{~B}$ & 0.0452 & 2.25 & \\
${ }^{12} \mathrm{C}$ & 0.0389 & 2.33 & \\
${ }^{28} \mathrm{Si}$ & 0.01 & 5.96 & \\
${ }^{51} \mathrm{~V}$ & 0.0125 & 4.478 & \\
${ }^{89} \mathrm{Y}$ & &
\end{tabular}

\begin{tabular}{|c|c|c|c|}
\hline $\begin{array}{l}\text { Target chamber } \\
\operatorname{Vacuum}\left(\sim 2 \times 10^{-5}[\text { Torr }], 20\left[{ }^{\circ} \mathrm{C}\right]\right)\end{array}$ & $\sim 81$ & $3.2 \times 10^{-11}$ & \\
\hline \multicolumn{4}{|l|}{ Spectrometer } \\
\hline $\operatorname{Vacuum}\left(\sim 2 \times 10^{-5}[\right.$ Torr $\left.], 20\left[{ }^{\circ} \mathrm{C}\right]\right)$ & $\sim 557$ & $3.2 \times 10^{-11}$ & \\
\hline Kevlar $\left(\mathrm{C}_{14} \mathrm{~N}_{2} \mathrm{O}_{2} \mathrm{H}_{10}\right)$ exit & 0.02 & 0.74 & \\
\hline Mylar $\left(\mathrm{C}_{10} \mathrm{O}_{4} \mathrm{H}_{8}\right)$ exit & 0.0125 & 1.39 & \\
\hline \multicolumn{4}{|l|}{ Detector Hut } \\
\hline$* \mathrm{He}$ bag & & & Placed between \\
\hline He gas & 100 & $1.79 \times 10^{-4}$ & HDC1 \& 2 \\
\hline Kapton bag & 0.004 & 1.42 & \\
\hline \multicolumn{4}{|l|}{$* \mathrm{HDC} 1$ and 2} \\
\hline DC Mylar cathode & 0.0176 & 1.39 & \\
\hline $\mathrm{Ar} / \mathrm{C}_{2} \mathrm{H}_{6}$ gas & 7.41 & 0.00154 & \\
\hline Sense wires $(\mathrm{W})$ & 0.00008 & 19.3 & \\
\hline Field wires (W) & 0.00034 & 19.3 & \\
\hline$*$ HTOF1X,1Y and $2 \mathrm{X}$ & 6 & 1.032 & \\
\hline \multicolumn{4}{|l|}{$* \mathrm{AC} 1,2$ and 3} \\
\hline Paper wall for AČ & 1.8 & 1.42 & \\
\hline Silica aerogel & 15 & 0.2 & \\
\hline \multicolumn{4}{|l|}{ *WČ 1 and 2} \\
\hline Plastic wall for WČ & 0.9 & 1.19 & Polymethylmethacrylate \\
\hline Water & 15 & 1 & \\
\hline \multicolumn{4}{|l|}{ *Miscellaneous } \\
\hline Air & $\sim 115$ & 0.0012 & HDC2 through WČ2 \\
\hline Aluminum foil for light shielding & 0.312 & 2.70 & \\
\hline Plastic sheet for light shielding & 1.04 & 1.30 & Polyvinyl-chloride \\
\hline
\end{tabular}


Table 4.8: Absorption factors depending on target. $\mathrm{K}^{+}$inelastic cross section $\left(\sigma_{\text {inel }}\right)$ is fixed as 10 mb.

\begin{tabular}{cc}
\hline Target & $\mathrm{K}^{+}$absorption factor \\
\hline \hline${ }^{\mathrm{CH}_{2}}$ & 0.9744 \\
${ }^{6} \mathrm{Li}$ & 0.9748 \\
${ }^{7} \mathrm{Li}$ & 0.9748 \\
${ }^{9} \mathrm{Be}$ & 0.9749 \\
${ }^{10} \mathrm{~B}$ & 0.9750 \\
${ }^{12} \mathrm{C}$ & 0.9750 \\
${ }^{28} \mathrm{Si}$ & 0.9750 \\
${ }^{51} \mathrm{~V}$ & 0.9750 \\
${ }^{89} \mathrm{Y}$ & 0.9750 \\
\hline
\end{tabular}

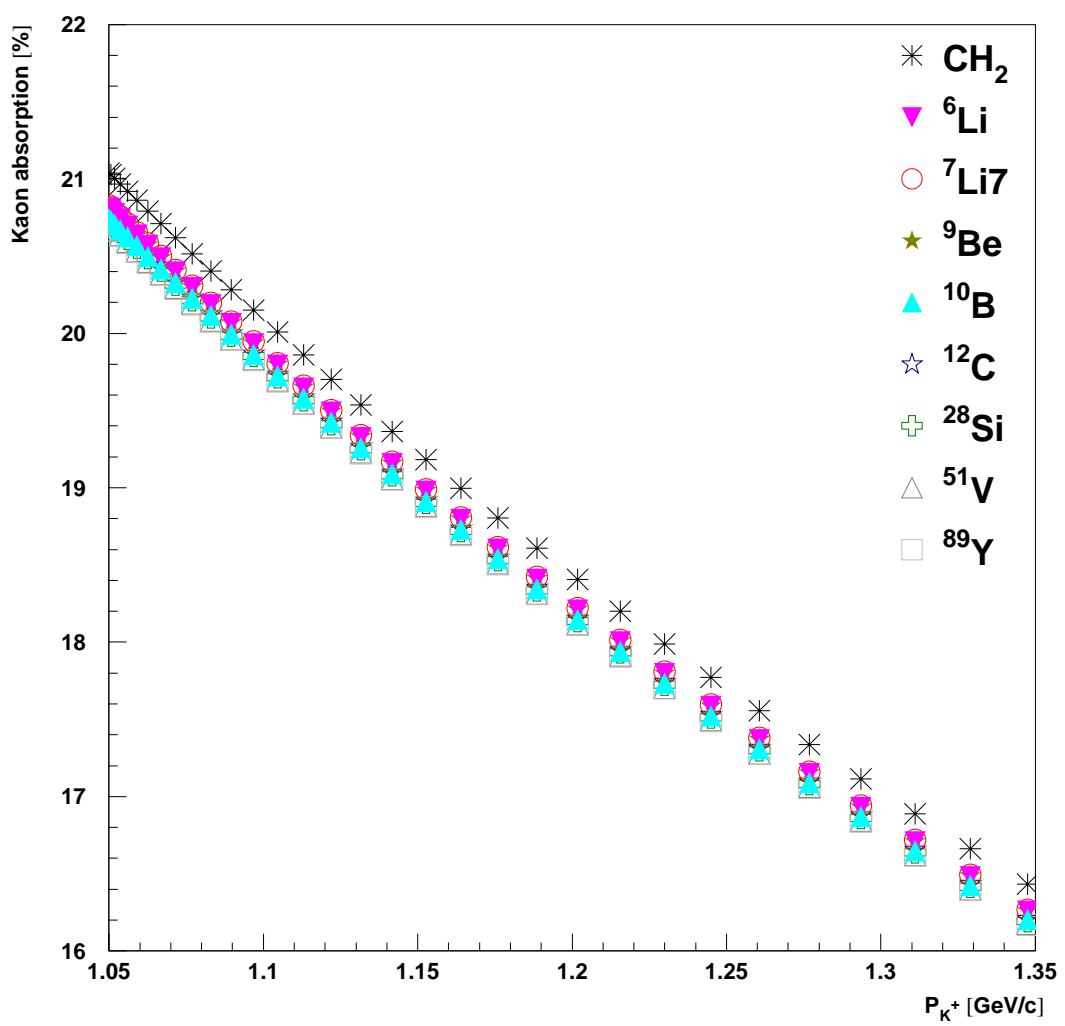

Figure 4.33: The momentum dependence of kaon absorption. 
Table 4.9: Summary of $\mathrm{K}^{+}$decay channels and branching ratios [82].

\begin{tabular}{cc}
\hline \hline Decay Modes & Brancing ratio [\%] \\
\hline$\mu^{+} \nu_{\mu}$ & $63.44 \pm 0.14$ \\
$\pi^{+} \pi^{0}$ & $20.92 \pm 0.12$ \\
$\pi^{+} \pi^{+} \pi^{-}$ & $5.59 \pm 0.03$ \\
$\pi^{0} e^{+} \nu_{\mathrm{e}}$ & $4.98 \pm 0.07$ \\
$\pi^{0} \mu^{+} \nu_{\mu}$ & $3.32 \pm 0.06$ \\
$\pi^{+} \pi^{0} \pi^{0}$ & $1.75 \pm 0.02$ \\
\hline \hline
\end{tabular}

The calculated decay factors of particles which were detected as 1) kaons (upper left), 2)
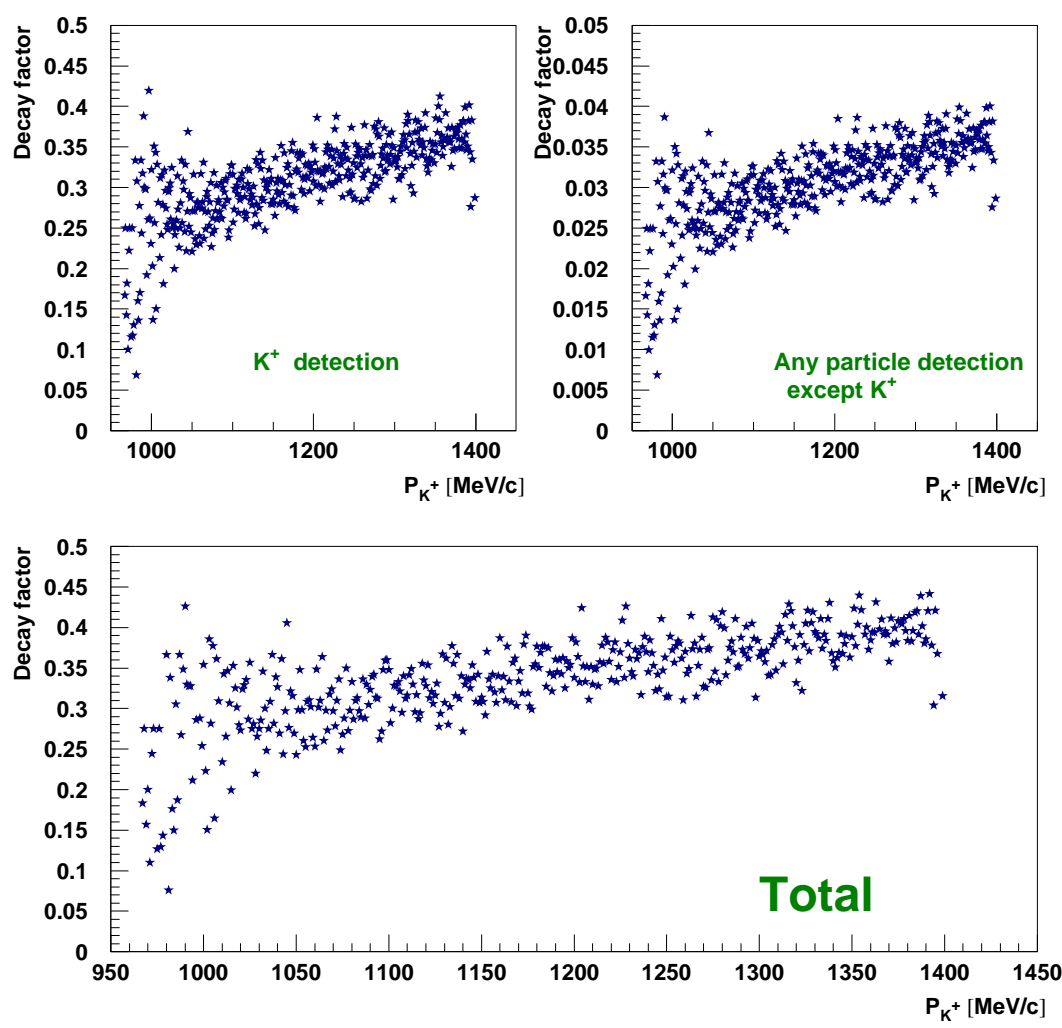

Figure 4.34: Momentum dependence of kaon decay factor.

particles except kaons (upper right) and 3) kaons involving decayed ones (lower) are represented in Fig. 4.34. The weighted average of the decay facter is estimated to be $34.5 \%$, and its error is dominated by statistics on the calculation as $0.01 \%$.

\section{Computer live time factor}

The computer live time factor is a ratio of a time interval when the DAQ processes a previous event. The Hall C DAQ processes the events with $\sim 400 \mu$ s time intervals or event rate $\sim 2.5$ $\mathrm{kHz}$. The computer live time factor $\left(f_{\text {comp }}\right)$ is calculated as a ratio of the number of pre-trigger and accepted trigger events as Eqn. 4.23. 


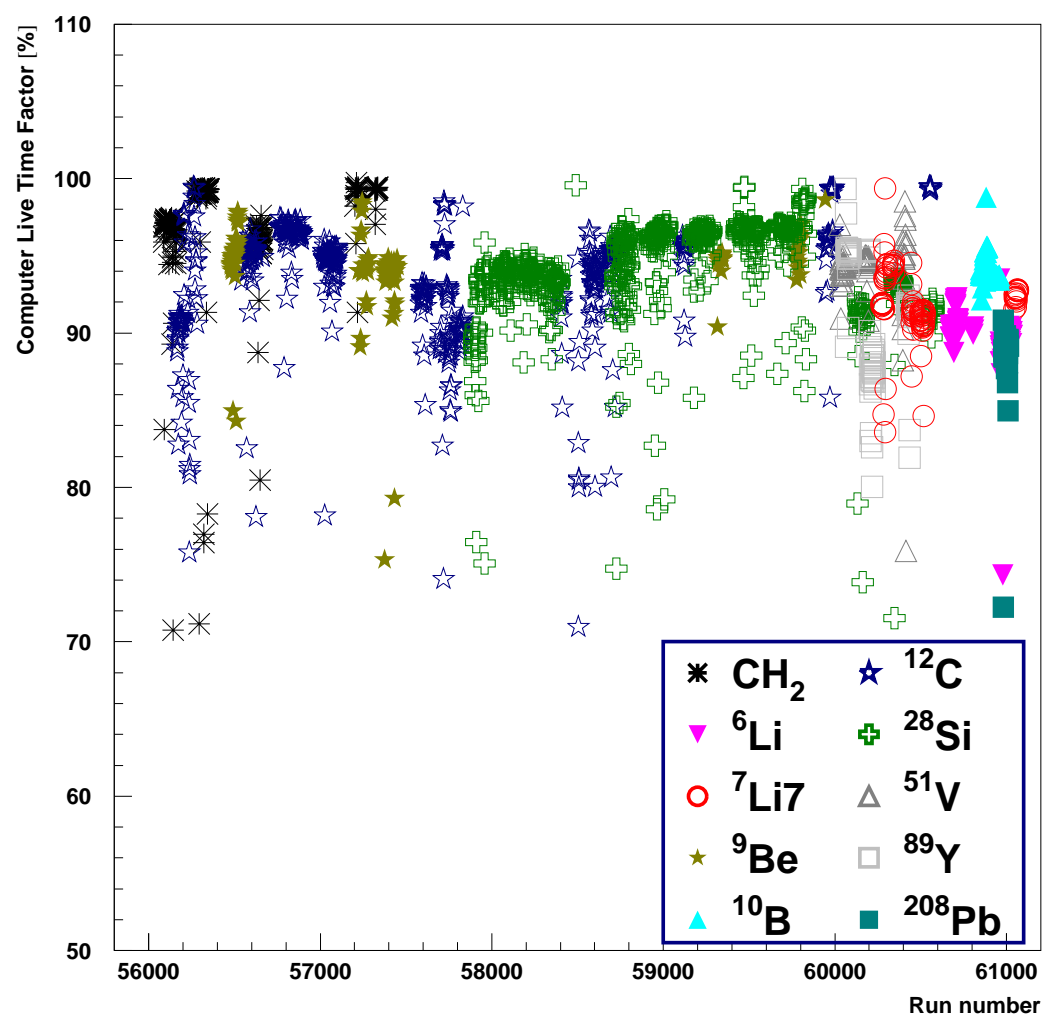

Figure 4.35: Computer live time factor.

Both accepted triggers and pre-triggers were recorded in scalers for all runs. Fig. 4.35 shows the computer live time versus run number.

$$
f_{\text {comp }}=\frac{\sum \text { trigger }}{\sum \text { pre-trigger }}
$$

\subsubsection{Systematic errors}

The total systematic errors of the cross section are summarized in Table 4.10. Details are described in the previous sections.

Table 4.10: A summary of systematic errors for the cross sections.

\begin{tabular}{|c|c|c|}
\hline \multirow[t]{2}{*}{ Item } & \multicolumn{2}{|c|}{ Systematic error $[\%]$} \\
\hline & ${ }^{7} \mathrm{Li}$ & ${ }^{12} \mathrm{C}$ \\
\hline Target thickness & 5 & 2 \\
\hline The number of virtual photons & \multicolumn{2}{|c|}{22} \\
\hline HKS acceptance & \multicolumn{2}{|c|}{1} \\
\hline Efficiency & \multicolumn{2}{|c|}{3} \\
\hline Total & 23 & 22 \\
\hline
\end{tabular}




\subsubsection{Energy resolution}

The energy resolution of the spectrometer system is derived from the FWHM of the measured ${ }_{\Lambda}^{12} \mathrm{~B}$ ground state. The deduced enery resolution is $0.49 \mathrm{MeV}$ (FWHM). The detailed description of this is discussed in Chapter 5. 



\section{Chapter 5}

\section{Experimental results and discussion}

A second generation $\Lambda$ hypernuclear spectroscopy experiment was successfully performed from June to September 2005 in the Hall C of Jefferson Laboratory.

Introducing the HKS spectrometer for the kaon arm, high-statistics and high energy-resolution spectroscopy of $\Lambda$ hypernuclei was realized.

In addition, the experiment demonstrated that the "tilt method" is a powerful technique to suppress background electrons due to Bremsstrahlung and Møller scattering, and thus accidental background.

In this chapter, the hypernuclear mass spectra of ${ }_{\Lambda}^{12} \mathrm{~B}$ and ${ }_{\Lambda}^{7} \mathrm{He}$ are presented and discussed. Cross sections of hypernuclear states were obtained and compared with some theoretical calculations.

\subsection{Experimental results}

\subsubsection{Hypernuclear mass spectra}

All of the data sets, which are used in the mass scale calibration and mass spectra evaluation, are summarized in Table 5.1 with trigger information.

The measured hypernuclear mass spectra of ${ }_{\Lambda}^{12} \mathrm{~B}$ and ${ }_{\Lambda}^{7} \mathrm{He}$ are shown in Figs. 5.1 and 5.2, respectively, with two kinds of vertical scales: the observed counts and the cross section,

$$
\overline{\left(\frac{d \sigma}{d \Omega}\right)_{1{ }^{\circ}-13^{\circ}}}=\frac{1}{N_{\mathrm{T}}} \frac{1}{N_{\gamma}} \sum_{i=1}^{N_{\mathrm{HY}}} \frac{1}{\epsilon_{\text {total }}(\Omega) d \Omega},
$$

where the symbols are defined in Section 4.7. The cross section is averaged over the HKS spectrometer angular acceptance, which covers $\mathrm{K}^{+}$scattering angles from $1^{\circ}$ to $13^{\circ}$, as listed in Table 2.1.

In the spectra presented in this chapter, two kinds of energy scales are given. One is the binding energy $\left(-B_{\Lambda}\right)$, defined by

$$
-B_{\Lambda}=M_{\mathrm{HY}}-\left(M_{\Lambda}+M_{A}\right),
$$

where $M_{\Lambda}, M_{A}$ and $M_{\mathrm{HY}}$ are the masses of a $\Lambda$, a core nucleus in its ground state and a hypernucleus, respectively. A summary of the nuclear masses, which were used for the present 
Table 5.1: Data sets used for the ${ }_{\Lambda}^{7} \mathrm{He}$ and ${ }_{\Lambda}^{12} \mathrm{~B}$ hypernuclear mass spectra.

\begin{tabular}{|c|c|c|c|c|c|}
\hline $\begin{array}{c}\text { Data set } \\
\text { No. }\end{array}$ & $\begin{array}{c}\text { Beam current } \\
\text { (Ave.) }[\mu \mathrm{A}]\end{array}$ & Target & Grouping & $\begin{array}{c}\text { Trigger } \\
\mathrm{ENGE} \times \text { HTOF } 1 \mathrm{X} \times \text { HTOF } 2 \mathrm{X} \times \alpha\end{array}$ & $\begin{array}{c}\text { Beam charge } \\
{[\mathrm{mC}]}\end{array}$ \\
\hline$\# 1$ & 2.3 & $\mathrm{CH}_{2}$ & OFF & 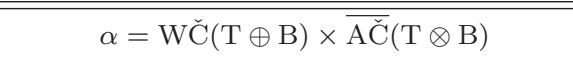 & 247.2 \\
\hline$\# 2$ & 16.2 & ${ }^{12} \mathrm{C}$ & OFF & $\alpha=\mathrm{W} \check{\mathrm{C}}(\mathrm{T} \oplus \mathrm{B}) \times \overline{\mathrm{AC}}(\mathrm{T} \otimes \mathrm{B})$ & 618.5 \\
\hline$\# 4$ & 19.3 & ${ }^{12} \mathrm{C}$ & $\mathrm{OFF}$ & $\alpha=\mathrm{W} \check{\mathrm{C}}(\mathrm{T} \otimes \mathrm{B}) \times \overline{\mathrm{AC}}(\mathrm{T} \oplus \mathrm{B})$ & 249.8 \\
\hline$\# 5$ & 2.3 & $\mathrm{CH}_{2}$ & $\mathrm{ON}$ & $\alpha=\mathrm{WC}(\mathrm{T} \otimes \mathrm{B}) \times \overline{\mathrm{AC}}(\mathrm{T} \oplus \mathrm{B})$ & 431.8 \\
\hline$\# 6$ & 24.5 & ${ }^{12} \mathrm{C}$ & $\mathrm{ON}$ & $\alpha=\mathrm{W} \check{\mathrm{C}}(\mathrm{T} \otimes \mathrm{B}) \times \overline{\mathrm{AC}}(\mathrm{T} \oplus \mathrm{B})$ & 6687.3 \\
\hline$\# 11$ & 21.3 & ${ }^{12} \mathrm{C}$ & $\mathrm{ON}$ & $\alpha=\mathrm{W} \check{\mathrm{C}}(\mathrm{T} \otimes \mathrm{B}) \times \overline{\mathrm{AC}}(\mathrm{T} \otimes \mathrm{B})$ & 1129.7 \\
\hline$\# 12$ & 21.6 & ${ }^{12} \mathrm{C}$ & $\mathrm{ON}$ & $\alpha=H T O F 1 Y \times \mathrm{WC}(\mathrm{T} \otimes \mathrm{B}) \times \overline{\mathrm{AC}}(\mathrm{T} \otimes \mathrm{B})$ & 661.3 \\
\hline$\# 14$ & 22.2 & ${ }^{12} \mathrm{C}$ & $\mathrm{ON}$ & $\alpha=H T O F 1 Y \times \mathrm{WC}(\mathrm{T} \oplus \mathrm{B}) \times \overline{\mathrm{AC}}(\mathrm{T} \otimes \mathrm{B})$ & 4633.1 \\
\hline$\# 19$ & 24.7 & ${ }^{7} \mathrm{Li}$ & $\mathrm{ON}$ & $\alpha=H T O F 1 Y \times \mathrm{WC}(\mathrm{T} \oplus \mathrm{B}) \times \overline{\mathrm{AC}}(\mathrm{T} \otimes \mathrm{B})$ & 3467.6 \\
\hline Total & $\begin{array}{l}- \\
- \\
-\end{array}$ & $\begin{array}{c}\mathrm{CH}_{2} \\
{ }^{7} \mathrm{Li} \\
{ }^{12} \mathrm{C}\end{array}$ & $\begin{array}{l}- \\
- \\
-\end{array}$ & $\begin{array}{l}- \\
- \\
-\end{array}$ & $\begin{array}{c}679.0 \\
3467.6 \\
13979.7\end{array}$ \\
\hline
\end{tabular}

spectra extraction, are given in Appendix A.

The other is the excitation energy of the hypernucleus with its origin defined as the ground state of each hypernucleus.

The accidental coincidence background is also overlaid both on the experimental counts (hatched with solid) and the cross section (accidental background level plus error bar) in Fig. 5.1 and 5.2 .

These accidental coincidence backgrounds are obtained from 4 bunches in the accidental coincidence regions as shown in Fig. 4.11. Therefore, the errors of the accidental coincidence events are smaller than those of the true timing events.

The quoted errors in Figs. 5.1 and 5.2 are statistical only.

Physics discussions of the results are given in Section 5.2. 


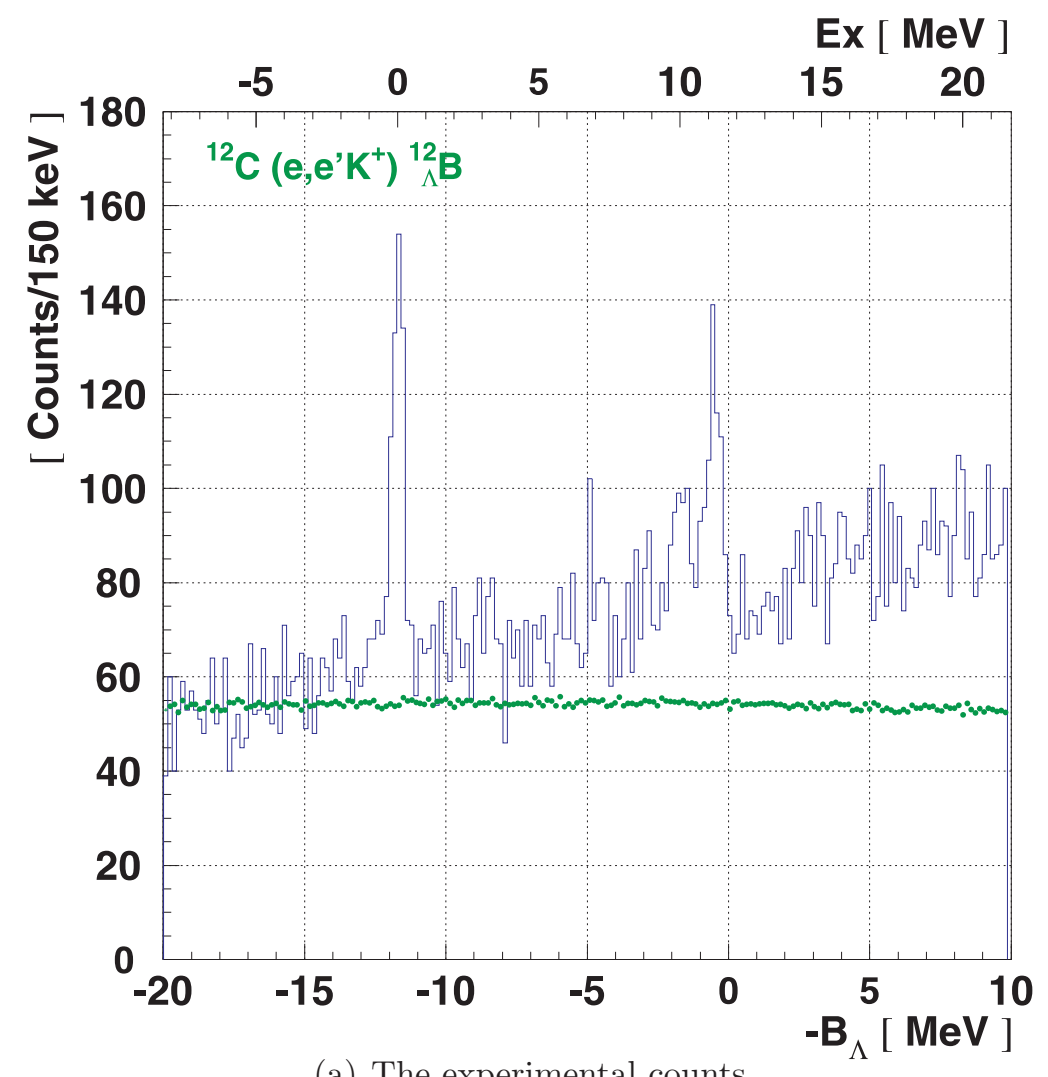

(a) The experimental counts

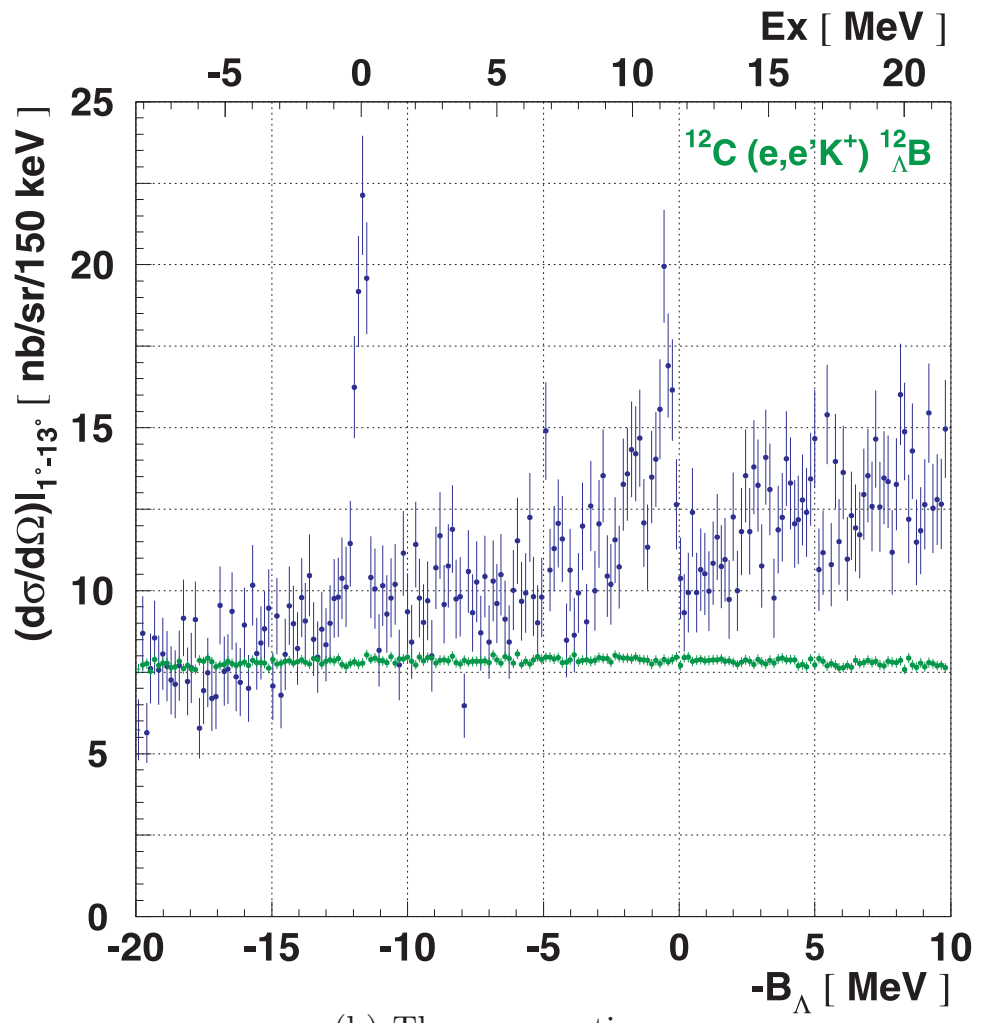

(b) The cross section

Figure 5.1: Hypernuclear mass spectra for ${ }_{\Lambda}^{12} \mathrm{~B}$. The errors are statistical only. 


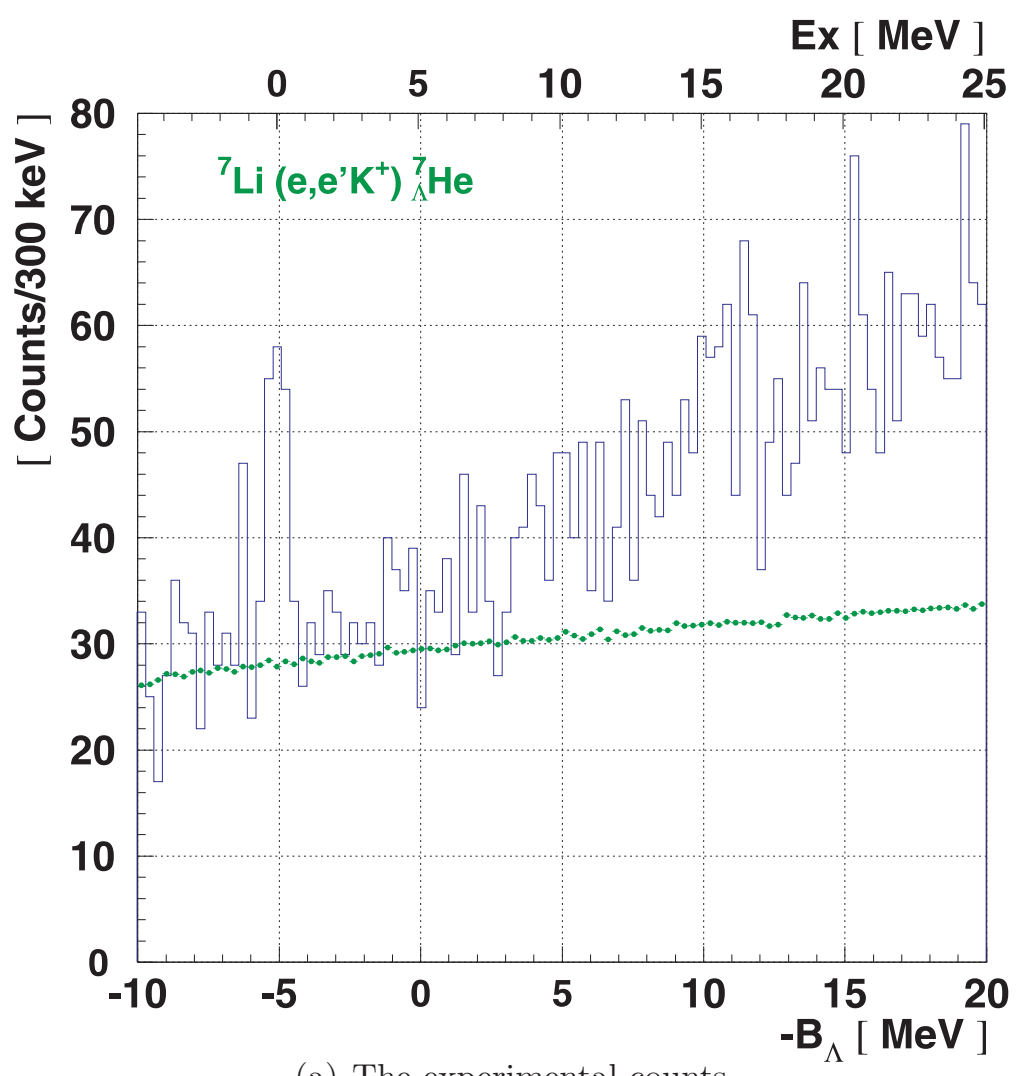

(a) The experimental counts
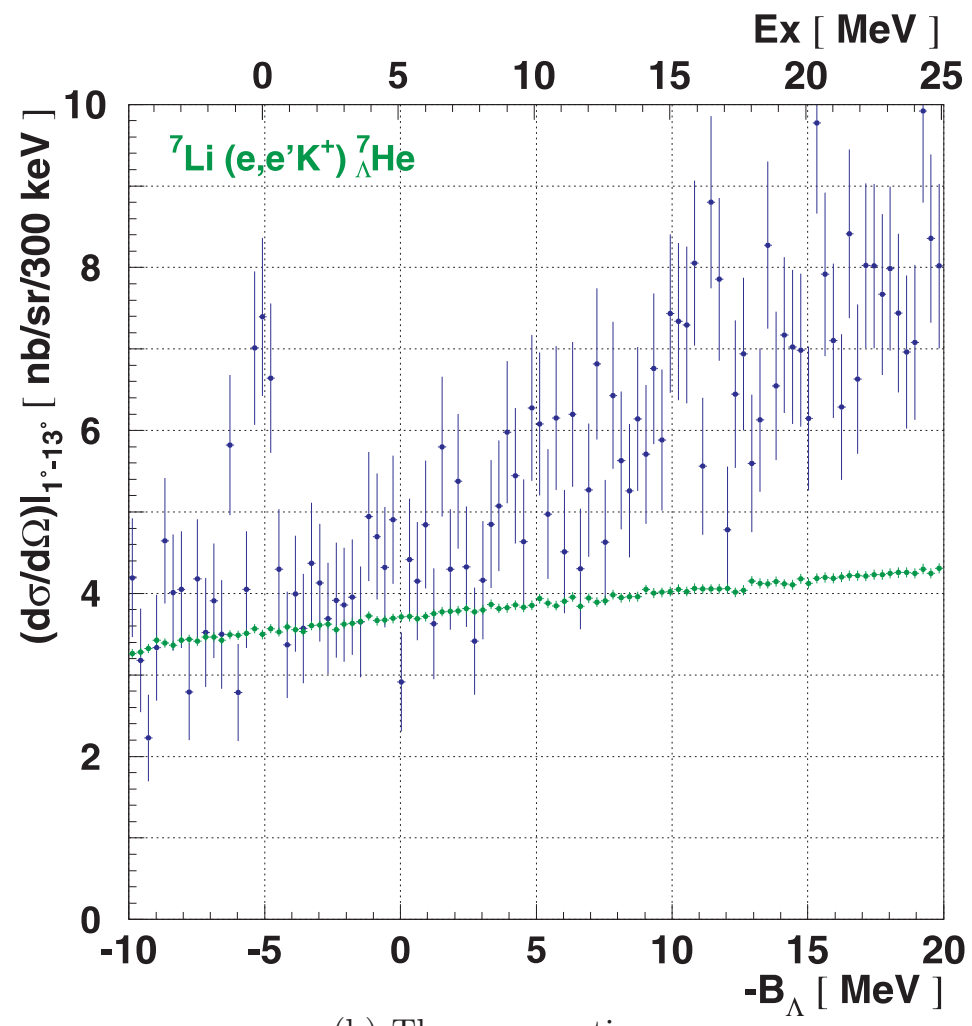

(b) The cross section

Figure 5.2: Hypernuclear mass spectra for ${ }_{\Lambda}^{7} \mathrm{He}$. The errors are statistical only. 


\subsubsection{Effectiveness of the present experimental configuration}

Before discussing the experimental results, let us remark upon the effectiveness of the "tilt method" and the introduction of the HKS.

The performance of the E01-011 and E89-009 experiments is compared item by item for a ${ }^{12} \mathrm{C}$ target and is summarized in Table 5.2.

The first 6 items from virtual photon flux per electron to averaged beam current in Table 5.2 are already represented in Chapter 4 for the E01-011 part. The equivalent values for the E89-009 experiments are taken from [83].

The experimental yield of the ${ }_{\Lambda}^{12} \mathrm{~B}$ ground state doublet above the accidental background is 451 counts for 180 hours in the E01-011 data taking in the present analysis while 165 counts were observed for approximately 440 hours in E89-009 [45].

The FWHM of the ${ }_{\Lambda}^{12} \mathrm{~B}$ ground state doublet for the present study is observed to be $490 \pm 70$ $\mathrm{keV}$ as represented later in Section 5.2.1 while $760 \mathrm{keV}$ was seen in E89-009 [46].

Table 5.2: Comparison of the measured performance between E01-011 and E89-009 experiments for ${ }^{12} \mathrm{C}$ targets.

\begin{tabular}{c|c|c|c}
\hline \hline Item & E01-011 & E89-009 & Gain factor \\
\hline \hline Virtual photon flux per electron $\left(\times 10^{-6}\right)$ & 4.8 & 370 & 0.01 \\
Target thickness $\left[\mathrm{mg} / \mathrm{cm}^{2}\right]$ & 100 & 22 & 4.5 \\
Scattered electron momentum acceptance $[\mathrm{MeV} / \mathrm{c}]$ & 320 & 120 & 2.6 \\
Kaon survival rate & 0.35 & 0.37 & 0.94 \\
Solid angle of $\mathrm{K}^{+}$arm $[\mathrm{msr}]$ & 16 & 4 & 4 \\
Beam current $[\mu \mathrm{A}]($ Averaged) & 20.2 & 0.66 & 40.4 \\
\hline \hline Yield rate of ${ }_{\Lambda}^{12} \mathrm{~B}$ ground state doublet $[\mathrm{Hz}]$ & $7.0 \times 10^{-4}$ & $1.0 \times 10^{-4}[45]$ & 7.0 \\
$\mathrm{~S} / \mathrm{N}$ ratio of ${ }_{\Lambda}^{2} \mathrm{~B}$ ground state doublet & 1.6 & 0.6 & 2.7 \\
Width of ${ }_{\Lambda}^{12} \mathrm{~B}$ ground state doublet $[\mathrm{keV}](\mathrm{FWHM})$ & $490 \pm 70$ & 760 & 0.6 \\
\hline \hline
\end{tabular}

As a reference, the experimental yield of ${ }_{\Lambda}^{12} \mathrm{~B}$ binding energy spectra which were observed in E89-009 and E01-011 experiments are compared in Fig. 5.3.

Comparing both $\mathrm{S} / \mathrm{N}$ ratio and energy resolution with the two experimental results in Fig. 5.3 , it can be said that the "tilt method" adoption for the scattering electron arm and the HKS spectrometer installation realized higher statistics and higher resolution $\Lambda$ hypernuclear spectroscopy.

\subsection{Discussion}

Seven years have passed since the first pioneering $\Lambda$ hypernuclear spectroscopic experiment with the $\left(\mathrm{e}, \mathrm{e}^{\prime} \mathrm{K}^{+}\right.$) reaction, JLab E89-009, was successfully performed. In E89-009, $\Lambda$ singleparticle states of ${ }_{\Lambda}^{7} \mathrm{He}[46]$ and ${ }_{\Lambda}^{12} \mathrm{~B}[45,84]$ were observed.

The present study considerably improved the ${ }_{\Lambda}^{12} \mathrm{~B}$ spectrum using the (e,e' $\left.\mathrm{K}^{+}\right)$reaction :

- the yield rate of ${ }_{\Lambda}^{12} \mathrm{~B}$ ground state doublet is increased by a factor of 7 ,

- the mass resolution (FWHM) is improved from $760 \mathrm{keV}$ to $490 \mathrm{keV}$,

- the $\mathrm{S} / \mathrm{N}$ ratio is improved by a factor of 2.7 . 


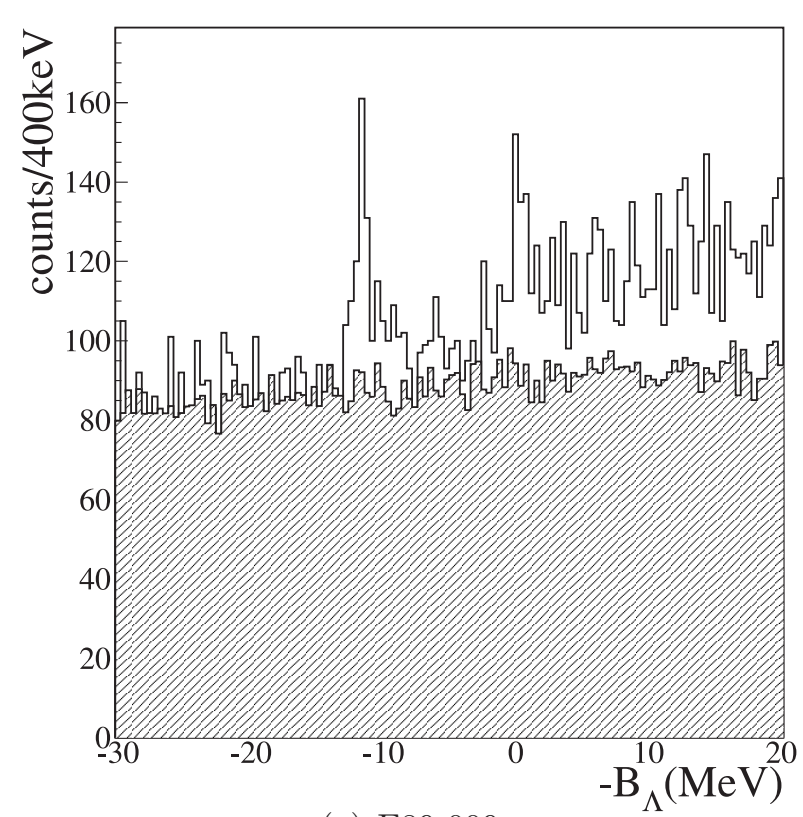

(a) E89-009

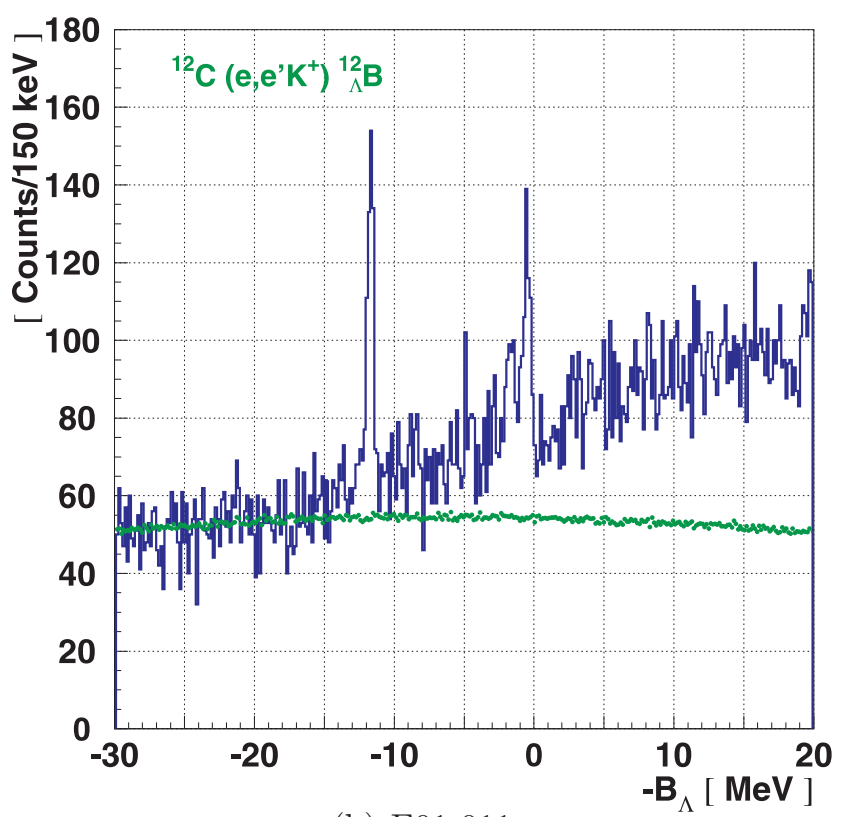

(b) E01-011

Figure 5.3: Comparison between E89-009 (a) and E01-011 (b) ${ }_{\Lambda}^{12} \mathrm{~B}$ spectra with the same energy scale in the bound region.

The results are compared with theoretical calculations in order to extract physics information.

The ${ }_{\Lambda}^{12} \mathrm{~B}$ results are discussed and the observed peak structure is also compared with another results measured in the JLab HallA experiment E94-107 [84] in section 5.2.1.

The experimental results of ${ }_{\Lambda}^{7} \mathrm{He}$ are discussed in section 5.2.2. The ${ }_{\Lambda}^{7} \mathrm{He}$ binding energy, which is measured for the first time by spectroscopic experiment, is represented. The energy scales and the cross sections are discussed with different theoretical models.

\subsubsection{Spectroscopy of ${ }_{\Lambda}^{12} \mathrm{~B}$ hypernuclei}

\section{Experimental result}

The hypernuclear mass spectrum of ${ }_{\Lambda}^{12} \mathrm{~B}$ measured by the $\left(\mathrm{e}, \mathrm{e}^{\prime} \mathrm{K}^{+}\right)$reaction after the background subtraction is shown in Fig. 5.4 (c). The width of the ground state doublet demonstrated an energy resolution of the present experiment to be $0.49 \pm 0.07 \mathrm{MeV}$ (FWHM). This is the best resolution ever achieved in hypernuclear reaction spectroscopy.

The ${ }_{\Lambda}^{12} \mathrm{~B}$ spectrum exhibits three prominent peaks at $-B_{\Lambda}$ of $\sim-11, \sim-1 \mathrm{MeV}$ marked as \#1, $\# 2$ and \#3 in Fig. 5.4 (c). These large peaks are interpreted as being proton-hole $\Lambda$-particle configurations, $\left[{ }^{11} \mathrm{~B}\left(3 / 2^{-} ; 0.00\right) \otimes s_{1 / 2 \Lambda}\right]$ (for $\left.\# 1\right)$ and $\left[{ }^{11} \mathrm{~B}\left(3 / 2^{-} ; 0.00\right) \otimes\left(p_{1 / 2 \Lambda}, p_{3 / 2 \Lambda}\right)\right]$ (for \#2 and \#3), respectively.

In addition, other peaks between $-B_{\Lambda}$ of $\sim-11$ and $-1 \mathrm{MeV}$ are statistically significant. However, none of significant peaks can be recognized in the present study, although some peaks were observed by the other $\Lambda$ hypernuclear spectroscopic experiment (E94-107) performed at Hall A, JLab in 2004, as shown in Fig. 5.4 (c) [84].

Considering that a ${ }_{\Lambda}^{12} \mathrm{~B}$ hypernucleus consists of a $\Lambda$ hyperon and a ${ }^{11} \mathrm{~B}$ core nucleus, there should be states which are interpreted to have excited core configurations with a $\Lambda$ hyperon as $\left[{ }^{11} \mathrm{~B}\left(1 / 2^{-} ; 2.12\right) \otimes s_{1 / 2 \Lambda}\right]$ and $\left[{ }^{11} \mathrm{~B}\left(3 / 2^{-} ; 5.02\right) \otimes s_{1 / 2 \Lambda}\right]$, respectively. In a proton knockout (e,e'p) reaction on ${ }^{12} \mathrm{C}$, the excited states of ${ }^{11} \mathrm{~B}$ at ${ }^{11} \mathrm{~B}\left(1 / 2^{-} ; 2.12 \mathrm{MeV}\right){ }^{11} \mathrm{~B}\left(3 / 2^{-} ; 5.02 \mathrm{MeV}\right.$ ) are populated with strength of $10-15 \%$ relative to the ground state (see Appendix B). Since 
the proton knockout reaction is analogous to the $\left(\mathrm{e}, \mathrm{e}^{\prime} \mathrm{K}^{+}\right)$reaction in the respect of that a proton hole is produced, the excess of events is considered to be related to such core excited states.

In order to extract the binding energies and cross sections of the observed major two peaks, the ${ }_{\Lambda}^{12} \mathrm{~B}$ spectrum is fitted with three Gaussians and a quasi-free component. The quasi-free component, representing a continuum spectrum in the unbound region, is fitted by a function proportional to the square root of $-B_{\Lambda}$. The quasi-free part is constrained to start at $B_{\Lambda}=0$ $\mathrm{MeV}$.

In the fitting for the three prominent peaks $(\# 1, \# 2$ and $\# 3)$, the widths are constrained to be the same.

The obtained binding energies and cross sections are presented in Table 5.3 and Fig. 5.4 (c). Refer to Appendix D for the detailed discussion on the systematic errors of the cross section for the ${ }_{\Lambda}^{12} \mathrm{~B}$ ground state doublet.

Table 5.3: Results of fitting the ${ }_{\Lambda}^{12} \mathrm{~B}$ spectrum.

\begin{tabular}{c|c|c|c}
\hline \hline $\begin{array}{c}\text { Peak } \\
\text { No. }\end{array}$ & $\begin{array}{c}-B_{\Lambda}[\mathrm{MeV}] \\
\text { (statistic) } \pm(\text { systematic })\end{array}$ & $\begin{array}{c}\text { FWHM } \\
{[\mathrm{MeV}]}\end{array}$ & $\begin{array}{c}\left.\overline{(d \sigma / d \Omega)}\right|_{1^{\circ}-13^{\circ}[\mathrm{nb} / \mathrm{sr}]} \\
(\text { statistic }) \pm(\text { systematic })\end{array}$ \\
\hline$\# 1$ & $-11.7 \pm 0.1 \pm 0.1$ & $0.49 \pm 0.07$ (fixed) & $54 \pm 10 \pm{ }_{22}^{25}$ \\
$\# 2$ & $-1.7 \pm 0.2 \pm 0.1$ & $0.49 \pm 0.07$ (fixed) & $31 \pm 8 \pm 7$ \\
$\# 3$ & $-0.5 \pm 0.1 \pm 0.1$ & $0.49 \pm 0.07$ (fixed) & $46 \pm 9 \pm 10$ \\
\hline \hline
\end{tabular}

\section{Comparison between the ${ }_{\Lambda}^{12} \mathrm{~B}$ and ${ }_{\Lambda}^{12} \mathrm{C}$ spectra}

The ${ }_{\Lambda}^{12} \mathrm{~B}$ and ${ }_{\Lambda}^{12} \mathrm{C}$ are mirror hypernuclei with each other. The observed ${ }_{\Lambda}^{12} \mathrm{~B}$ spectrum can be compared with the latest ${ }_{\Lambda}^{12} \mathrm{C}$ hypernucleus spectrum from the $\left(\pi^{+}, \mathrm{K}^{+}\right)$reaction (Fig. 5.4 (a)) which was measured by the superconducting kaon spectrometer (SKS) with $p_{\pi^{+}}=1.06 \mathrm{GeV} / c$ of $\pi^{+}$beam momentum at the K6 beamline of the KEK 12 GeV PS in 2001 (KEK-E369) [31]. Two prominent peaks (\#1 and \#5) at $-B_{\Lambda}=-10.76$ and $-0.10 \mathrm{MeV}$ and four smaller peaks $(\# 2, \# 3, \# 4$ and $\# 6$ ) were observed together with the quasi-free contribution in the unbound region due to the higher statistics, although the estimated energy resolution was $1.5-2.0 \mathrm{MeV}$ (FWHM).

The measured excitation energies, binding energies and cross sections are summarized in Table $5.4[31]$.

The two large peaks (\#1 and \#5) are interpreted to have neutron-hole $\Lambda$-particle configurations, $\left[{ }^{11} \mathrm{C}\left(3 / 2^{-} ; 0.00\right) \otimes s_{1 / 2 \Lambda}\right]$ and $\left[{ }^{11} \mathrm{C}\left(3 / 2^{-} ; 0.00\right) \otimes\left(p_{1 / 2 \Lambda}, p_{3 / 2 \Lambda}\right)\right]$, respectively. Configurations of these two peaks are analogous to those of ${ }_{\Lambda}^{12} \mathrm{~B}$ which have proton-hole $\Lambda$-particle configurations, respectively.

The $10.66 \pm 0.04$ (stat.) MeV spacing between the \#1 and \#5 peaks in the ${ }_{\Lambda}^{12} \mathrm{C}$ mass spectrum is compared to the $11.2 \pm 0.1$ (stat.) \pm 0.1 (syst.) $\mathrm{MeV}$ spacing of ${ }_{\Lambda}^{12} \mathrm{~B}$ (\#1 and \#3). The energy difference is $0.5 \pm 0.2 \mathrm{MeV}$ as listed in Table 5.3.

The energy difference of the $s-p$ states spacing $(\Delta \mathrm{sp})$ between charge symmetric ${ }_{\Lambda}^{12} \mathrm{~B}$ and ${ }_{\Lambda}^{12} \mathrm{C}$ is consistent with the theoretically calculated value of $0.46 \mathrm{MeV}$ as listed in Table 5.5. 


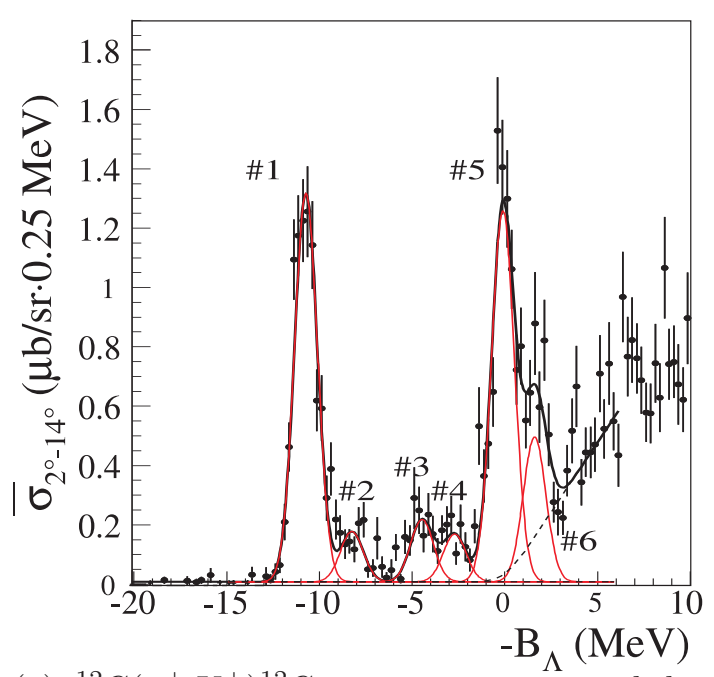

(a) ${ }^{12} \mathrm{C}\left(\pi^{+}, \mathrm{K}^{+}\right){ }_{\Lambda}^{12} \mathrm{C}$ spectrum measured by KEK-E369 [31] with $1.06 \mathrm{GeV} / c$ of $\pi^{+}$beam momentum. The energy resolution was $\leq 2.0$ $\mathrm{MeV}$.

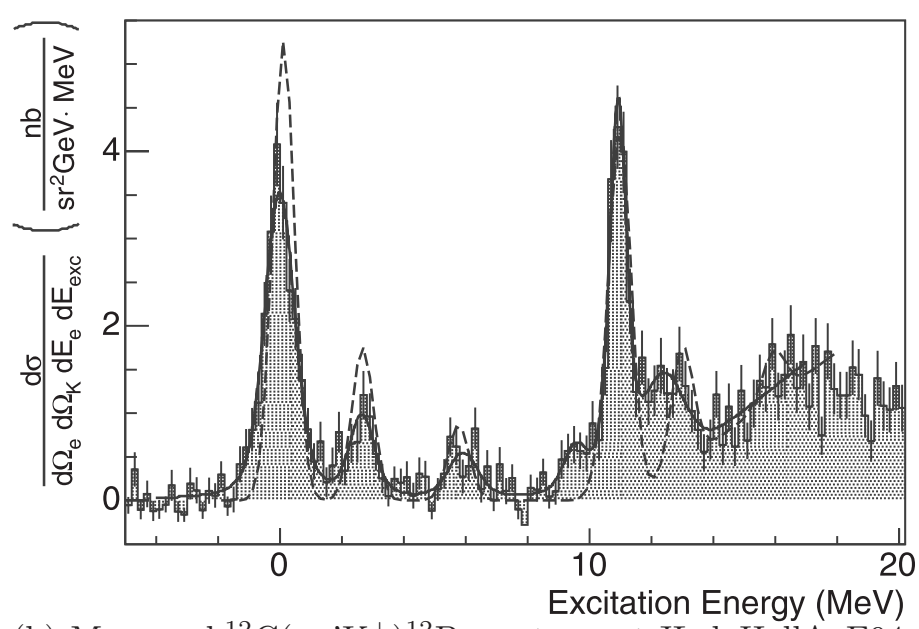

(b) Measured ${ }^{12} \mathrm{C}\left(\mathrm{e}, \mathrm{e}^{\prime} \mathrm{K}^{+}\right)_{\Lambda}^{12} \mathrm{~B}$ spectrum at JLab HallA, E94107 [84].

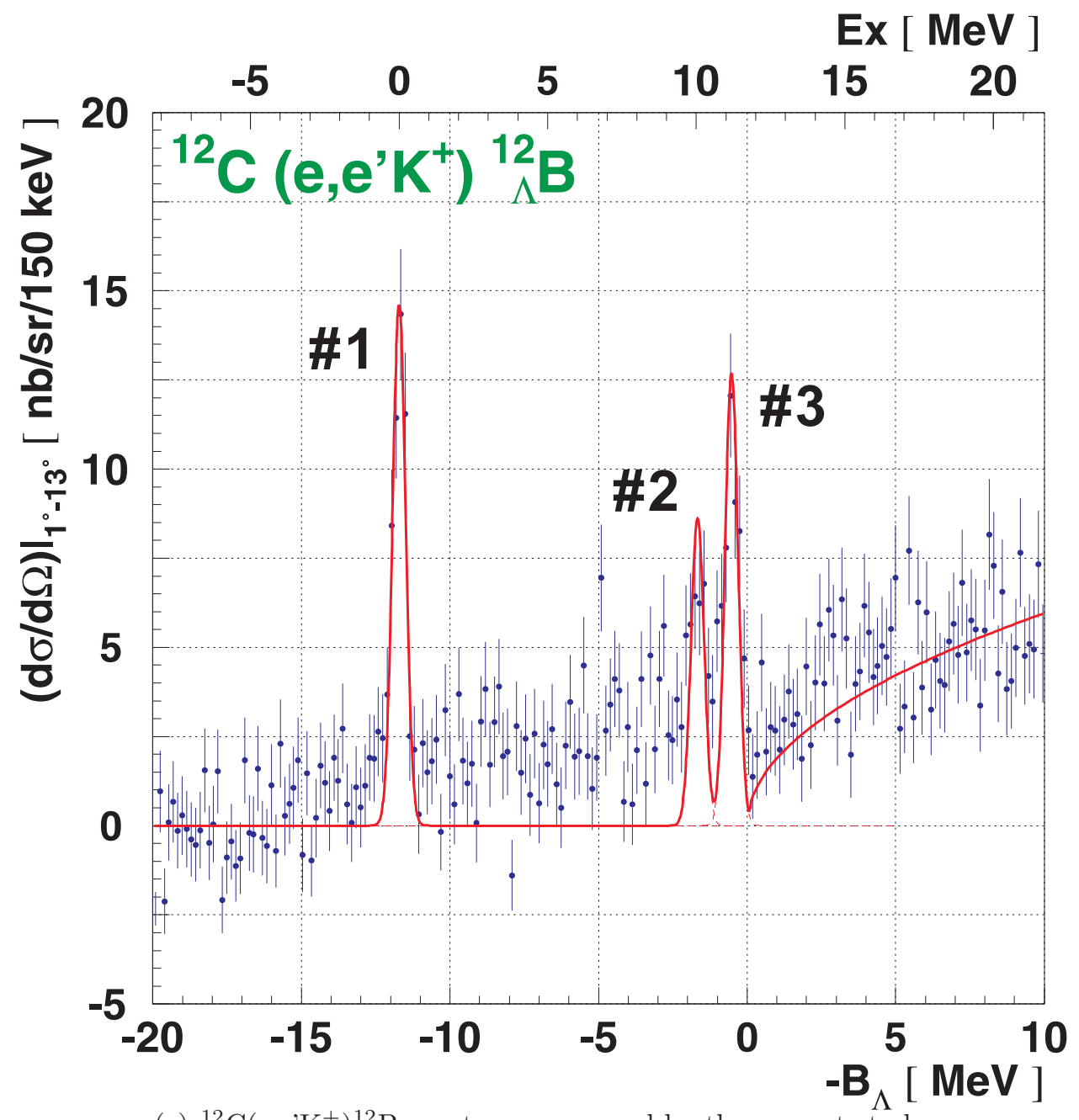

(c) ${ }^{12} \mathrm{C}\left(\mathrm{e}, \mathrm{e}^{\prime} \mathrm{K}^{+}\right){ }_{\Lambda}^{12} \mathrm{~B}$ spectrum measured by the present study.

Figure 5.4: The hypernuclear mass spectra of ${ }_{\Lambda}^{12} \mathrm{C}$ via the $\left(\pi^{+}, \mathrm{K}^{+}\right)$reaction by KEK-SKS E369 (a), ${ }_{\Lambda}^{12} \mathrm{~B}$ at JLab HallA by E94-107, and ${ }_{\Lambda}^{12} \mathrm{~B}$ by the present study (c). 
Table 5.4: Results of the fitting the ${ }_{\Lambda}^{12} \mathrm{C}$ spectrum obtained with the thin target, where $E_{x}$ shows the excitation energy. The quoted errors are statistical [31].

\begin{tabular}{cccccc}
\hline \hline $\begin{array}{c}\text { Peak } \\
\text { No. }\end{array}$ & $\begin{array}{c}E_{x} \\
{[\mathrm{MeV}]}\end{array}$ & $\begin{array}{c}B_{\Lambda} \\
{[\mathrm{MeV}]}\end{array}$ & $\begin{array}{c}\text { Errors } \\
{[\mathrm{MeV}]}\end{array}$ & $\begin{array}{c}\text { FWHM } \\
{[\mathrm{MeV}]}\end{array}$ & $\begin{array}{c}\text { Cross sections } \\
{[\mu \mathrm{b} / \mathrm{sr}]}\end{array}$ \\
\hline$\# 1$ & & 10.76 & (fixed) & $1.44 \pm 0.05$ & $8.07 \pm 0.38$ \\
$\# 2$ & 2.51 & 8.25 & \pm 0.17 & $1.44 \pm 0.05$ & $1.04 \pm 0.14$ \\
$\# 3$ & 6.30 & 4.46 & \pm 0.11 & $1.44 \pm 0.05$ & $1.29 \pm 0.21$ \\
$\# 4$ & 8.06 & 2.70 & \pm 0.19 & $1.44 \pm 0.05$ & $0.99 \pm 0.17$ \\
$\# 5$ & 10.66 & 0.10 & \pm 0.04 & $1.44 \pm 0.05$ & $7.71 \pm 0.45$ \\
$\# 6$ & 12.37 & -1.61 & \pm 0.09 & $1.44 \pm 0.05$ & $3.01 \pm 0.40$ \\
\hline \hline
\end{tabular}

Table 5.5: Measured $s-p$ states energy spacing obtained from the ${ }_{\Lambda}^{12} \mathrm{C}$ and ${ }_{\Lambda}^{12} \mathrm{~B}$ excitation spectra shown with the theoretical prediction.

\begin{tabular}{c|c|c|c|c}
\hline \hline \multirow{2}{*}{ Reaction } & \multicolumn{2}{|c|}{ Experiment } & \multicolumn{2}{|c}{ Calculation } \\
\cline { 2 - 5 } & $\begin{array}{c}\Delta \mathrm{sp} \\
{[\mathrm{MeV}]}\end{array}$ & $\begin{array}{c}\Delta \mathrm{sp}\left({ }_{\Lambda}^{12} \mathrm{~B}\right)-\Delta \mathrm{sp}\left({ }_{\Lambda}^{12} \mathrm{C}\right) \\
{[\mathrm{MeV}]}\end{array}$ & $\begin{array}{c}\Delta \mathrm{sp} \\
{[\mathrm{MeV}]}\end{array}$ & $\begin{array}{c}\Delta \mathrm{sp}\left({ }_{\Lambda}^{12} \mathrm{~B}\right)-\Delta \mathrm{sp}\left({ }_{\Lambda}^{12} \mathrm{C}\right) \\
{[\mathrm{MeV}]}\end{array}$ \\
\hline${ }^{12} \mathrm{C}\left(\mathrm{e}, \mathrm{e}^{\prime} \mathrm{K}^{+}\right){ }_{\Lambda}^{12} \mathrm{~B}$ & $11.2 \pm 0.1$ & $0.5 \pm 0.2$ & $11.06[85]$ & 0.46 \\
${ }^{12} \mathrm{C}\left(\mathrm{e}, \mathrm{e}^{\prime} \mathrm{K}^{+}\right){ }_{\Lambda}^{12} \mathrm{C}$ & $10.66 \pm 0.04$ & $10.6[33]$ & \\
\hline \hline
\end{tabular}

\section{Theoretical interpretation of the ${ }^{12} \mathrm{C}\left(\mathrm{e}, \mathrm{e}^{\prime} \mathbf{K}^{+}\right){ }_{\Lambda}^{12} \mathrm{~B}$ reaction}

The production and structure of $p$-shell $\Lambda$ hypernuclei were investigated by Itonaga et al. $[32,33]$ with configuration-mixed shell model wave functions which were established based on the experimental data from the $\left(\mathrm{K}^{-}, \pi^{-}\right)$and $\left(\pi^{+}, \mathrm{K}^{+}\right)$reactions.

They calculated the hypernuclear properties by diagonalizing the Hamiltonian (more precise description of Eqn. 1.1):

$$
H=H_{\mathrm{N}}^{(\text {Cohen-Kurath })}+t_{\Lambda}+\xi \boldsymbol{l}_{\Lambda} \cdot \boldsymbol{s}_{\Lambda}+\sum_{\mathrm{N}} v_{\Lambda \mathrm{N}}
$$

where $t_{\Lambda}$ is the kinetic energy of a $\Lambda$ hyperon, $\xi \boldsymbol{l}_{\Lambda} \cdot \boldsymbol{s}_{\Lambda}$ is the $\Lambda$ spin-orbit potential, and $v_{\Lambda \mathrm{N}}$ is the $\Lambda \mathrm{N}$ interaction. For the core-nucleus part, the Cohen-Kurath shell-model Hamiltonian $\left(H_{\mathrm{N}}^{(\text {Cohen-Kurath) }}\right)$ and its wave functions [6] are adopted, since spectroscopic factors of the $p$-shell nuclei are generally well reproduced by the Cohen-Kurath's shell model calculation $[6,86,87]$. For the interaction part, the YNG interaction [11] deduced on the basis of the G-matrix calculation with the Nijmegen soft core model (NSC97f) [88] interaction is used. In treating the reaction, the DWIA formalism is adopted as discussed in Section 1.5.2.

The calculated spectrum of the ${ }_{\Lambda}^{12} \mathrm{~B}$ by $\left(\gamma, \mathrm{K}^{+}\right)$reaction at $E_{\gamma}=1.3 \mathrm{GeV}$ and $\theta_{\mathrm{K}^{+}}^{\mathrm{LAB}}=3^{\circ}$ with a fixed energy resolution of $300 \mathrm{keV}$ (FWHM) is shown in Fig. 5.5 and the corresponding values of the cross sections and excitation energies are listed in Table 5.6.

The experimental results of the ${ }_{\Lambda}^{12} \mathrm{~B}$ cross sections and excitation energies are compared to theoretical predictions by Sotona et al. in Table 5.7 with three different models ; Saclay-Lyon (SLA) [90], Williams-Ji-Cotanch (C4) [91], and Kaon-MAID (KMAID) [92]. The cross sections 


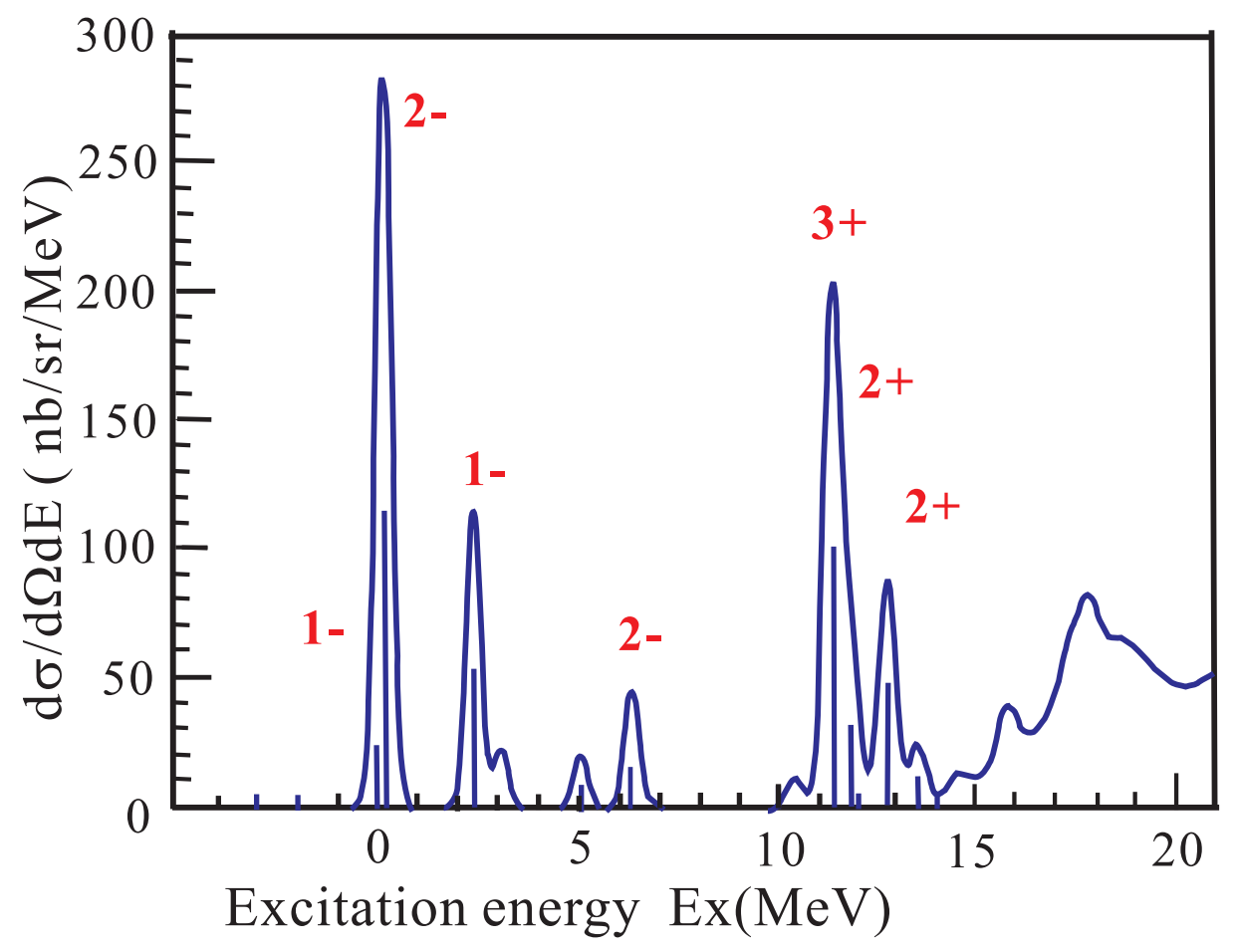

Figure 5.5: Calculated spectrum for the ${ }^{12} \mathrm{C}\left(\gamma, \mathrm{K}^{+}\right)_{\Lambda}^{12} \mathrm{~B}$ reaction at $E_{\gamma}=1.3 \mathrm{GeV}$ and $\theta_{\mathrm{K}^{+}}^{\mathrm{LAB}}=3^{\circ}[89]$.

Table 5.6: Comparison of obtained excitation energies and cross sections with theoretical calculations for ${ }_{\Lambda}^{12} \mathrm{~B}$ for the virtual photon energy of $E_{\gamma}=1.3 \mathrm{GeV}$.

\begin{tabular}{|c|c|c|c|c|c|}
\hline \multirow{2}{*}{$\begin{array}{c}\text { Peak } \\
\text { No. }\end{array}$} & \multicolumn{2}{|c|}{ Experimental results } & \multicolumn{3}{|c|}{ Theoretical calculation } \\
\hline & $\begin{array}{c}\mathrm{Ex} \\
{[\mathrm{MeV}]}\end{array}$ & $\begin{array}{c}\left.\overline{(d \sigma / d \Omega)}\right|_{1^{\circ}-13^{\circ}} \\
{[\mathrm{nb} / \mathrm{sr}]}\end{array}$ & Main structure $: J^{\pi}$ & $\begin{array}{c}\mathrm{Ex} \\
{[\mathrm{MeV}]}\end{array}$ & $\begin{array}{c}\text { Cross section } \\
{[\mathrm{nb} / \mathrm{sr}]}\end{array}$ \\
\hline \# 1 & 0.0 & $54 \pm 10 \pm{ }_{22}^{25}$ & $\begin{array}{l}{\left[{ }^{11} \mathrm{~B}\left(3 / 2^{-} ; \text {g.s. }\right) \otimes s_{1 / 2 \Lambda}\right]: 1^{-}} \\
{\left[{ }^{11} \mathrm{~B}\left(3 / 2^{-} ; \text {g.s. }\right) \otimes s_{1 / 2 \Lambda}\right]: 2^{-}}\end{array}$ & $\begin{array}{l}0.00 \\
0.14\end{array}$ & $\begin{array}{c}34.9 \\
120.8\end{array}$ \\
\hline$\# 2$ & $10.0 \pm 0.2$ & & {$\left[{ }^{11} \mathrm{~B}\left(3 / 2^{-} ;\right.\right.$g.s. $\left.) \otimes p_{3 / 2 \Lambda}\right]: 3^{+}$} & 10.08 & 98.3 \\
\hline$\# 3$ & $11.2 \pm 0.1 \pm 0.1$ & $46 \pm 9 \pm 10$ & {$\left[{ }^{11} \mathrm{~B}\left(3 / 2^{-} ;\right.\right.$g.s. $\left.) \otimes p_{1 / 2 \Lambda}\right]: 2^{+}$} & 10.61 & 61.5 \\
\hline
\end{tabular}

Table 5.7: Comparison of obtained excitation energies and cross sections with theoretical calculations for ${ }_{\Lambda}^{12} \mathrm{~B}$ for the virtual photon energies of $1.3<E_{\gamma}<1.6 \mathrm{GeV}$ corresponding to the present experiment.

\begin{tabular}{|c|c|c|c|c|c|c|c|}
\hline & \multicolumn{2}{|c|}{ Experimental results } & \multicolumn{5}{|c|}{ Theoretical prediction } \\
\hline \multirow{2}{*}{$\begin{array}{c}\text { peak } \\
\text { no. }\end{array}$} & \multirow{2}{*}{$\begin{array}{c}\mathrm{Ex} \\
{[\mathrm{MeV}]}\end{array}$} & \multirow{2}{*}{$\begin{array}{c}\left.\overline{(d \sigma / d \Omega)}\right|_{1^{\circ}-13^{\circ}} \\
{[\mathrm{nb} / \mathrm{sr}]}\end{array}$} & \multirow[t]{2}{*}{$J^{\pi}$} & \multirow{2}{*}{$\begin{array}{c}\mathrm{Ex} \\
{[\mathrm{MeV}]}\end{array}$} & \multicolumn{3}{|c|}{$\left.\overline{(d \sigma / d \Omega)}\right|_{1^{\circ}-13^{\circ}[\mathrm{nb} / \mathrm{sr}]}$} \\
\hline & & & & & SLA & $\mathrm{C} 4$ & KMAID \\
\hline \multirow[t]{2}{*}{ \#\# 1} & \multirow[t]{2}{*}{0.0} & \multirow{2}{*}{$\overline{c 54 \pm 10 \pm{ }_{22}^{25}}$} & $\overline{1}^{-}$ & $\overline{0} 0$ & 19.7 & 22.8 & 20.7 \\
\hline & & & $2^{-}$ & 0.14 & 65.7 & 82.0 & 43.0 \\
\hline$\# 2$ & $10.0 \pm 0.2 \pm 0.1$ & $31 \pm 8 \pm 7$ & $2^{+}$ & 10.99 & 48.3 & 56.9 & 38.0 \\
\hline$\# 3$ & $11.2 \pm 0.1 \pm 0.1$ & $46 \pm 9 \pm 10$ & $3^{+}$ & 11.06 & 75.3 & 107.3 & 68.5 \\
\hline
\end{tabular}


for the virtual photon energies of $1.3<E_{\gamma}<1.6 \mathrm{GeV}$ and the kaon scattering angles of $1^{\circ}<$ $\theta_{\mathrm{K}^{+}}^{\mathrm{Lab}}<13^{\circ}$, which corresponds to the present experimental condition with a beam energy of $E_{\text {beam }}=1.8 \mathrm{GeV}$, are averaged for each theoretical calculation.

It is found that the calculation with the KMAID model reproduces the experimental results well compared with the other two models.

\section{Binding energy of the ${ }_{\Lambda}^{12} \mathbf{B}$ ground state doublet}

The binding energy of the ${ }_{\Lambda}^{12} \mathrm{~B}$ ground state doublet is observed to be $11.7 \pm 0.1$ (stat) \pm 0.1 (syst) $\mathrm{MeV}$, which is consistent with the previous experimental result of $11.52 \pm 0.35 \mathrm{MeV}$ [46] and the emulsion result of $11.37 \pm 0.06 \mathrm{MeV}$ [57]. Here, the latter result was evaluated from 87 observed events for the decay process of ${ }_{\Lambda}^{12} \mathrm{~B} \rightarrow \pi^{-}+\alpha+\alpha+\alpha$ and the quoted error is statistical only. 


\subsubsection{Spectroscopy of ${ }_{\Lambda}^{7} \mathrm{He}$ hypernuclei}

\section{Significance of the ${ }_{\Lambda}^{7} \mathrm{He}$ binding energy measurement}

The significance of the ${ }_{\Lambda}^{7}$ He excitation energy measurement can be summarized in the following two points.

\section{1) Investigation of the $\Lambda \mathrm{NN}$ force}

Information on the $\Lambda \mathrm{NN}$ three-body force is expected to be derived from the experimental data of the neutron-rich ${ }_{\Lambda}^{7} \mathrm{He}$ binding energy, since the coherent $\Lambda \mathrm{N}-\Sigma \mathrm{N}$ coupling effect is expected to be more significant in neutron-rich $\Lambda$ hypernuclei [93].

So far, there has existed a long standing problem that there is no $\Lambda \mathrm{N}$ interaction which reproduces the binding energies $\left(B_{\Lambda}\right)$ of the simple $s$-shell hypernuclei, ${ }_{\Lambda}^{3} \mathrm{H},{ }_{\Lambda}^{4} \mathrm{H},{ }_{\Lambda}^{4} \mathrm{He}$ and ${ }_{\Lambda}^{5} \mathrm{He}$, simultaneously. Dalitz et al. [94] used a reasonable $\Lambda \mathrm{N}$ central force which reproduces the correct binding energies for ${ }_{\Lambda}^{3} \mathrm{H}$ and ${ }_{\Lambda}^{4} \mathrm{H}$, but that potential gives the overbinding result of $B_{\Lambda}=5.46 \mathrm{MeV}$ for the ${ }_{\Lambda}^{5} \mathrm{He}$ ground state, which is much larger than the observed value of 3.12 $\pm 0.02 \mathrm{MeV}$ by an emulsion experiment [57]. Akaishi et al. pointed out that the problem is not in ${ }_{\Lambda}^{5} \mathrm{He}$, but in the treatment of the $\Lambda \mathrm{N}$ interaction for the ${ }_{\Lambda}^{4} \mathrm{H}$ and ${ }_{\Lambda}^{4} \mathrm{He}$ system [95]. The experimental data for ${ }_{\Lambda}^{4} \mathrm{He}$ was reproduced together with ${ }_{\Lambda}^{3} \mathrm{H}$ and ${ }_{\Lambda}^{5} \mathrm{He}$ by introducing correctly the coherent $\Lambda \mathrm{N}-\Sigma \mathrm{N}$ coupling effect which gives rise to the $\Lambda \mathrm{NN}$ three-body force as shown in Fig. 5.6.

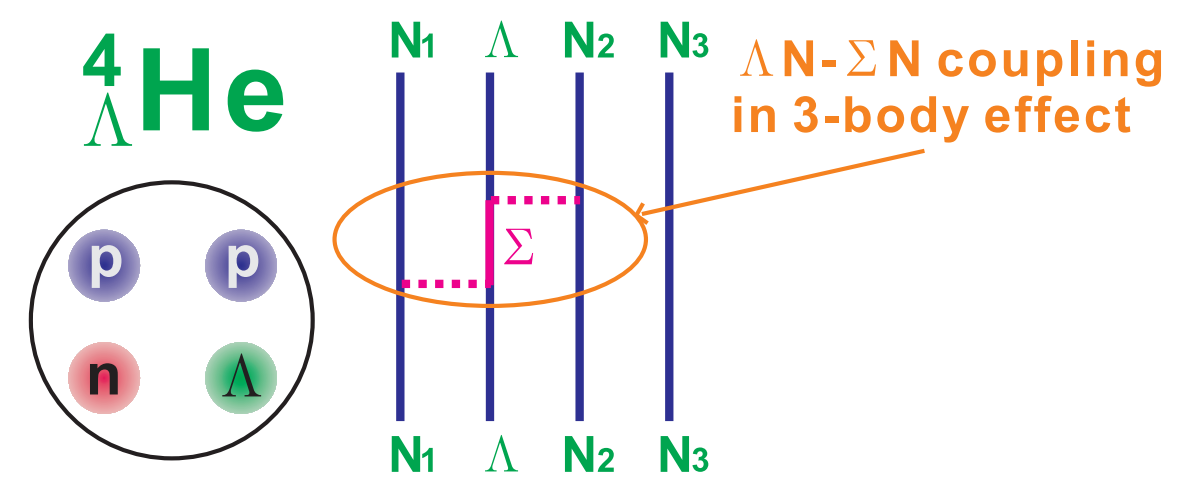

Figure 5.6: Description of the $\Lambda \mathrm{N}-\Sigma \mathrm{N}$ coupling in three-body interaction on ${ }_{\Lambda}^{4} \mathrm{He}$.

Since the coherent $\Lambda \mathrm{N}-\Sigma \mathrm{N}$ coupling effect plays a significant role in neutron-rich $\Lambda$ hypernuclei [93], the ${ }_{\Lambda}^{7} \mathrm{He}$ binding energy, which has never been measured, is expected to provide further information on the $\Lambda \mathrm{NN}$ three-body force.

\section{2) Verification of the charge symmetry breaking}

The large energy difference between the ground state of ${ }_{\Lambda}^{4} \mathrm{He}$ and that of ${ }_{\Lambda}^{4} \mathrm{H}\left(\Delta E_{\text {Exp. }}=\right.$ $2.39-2.04=0.35 \mathrm{MeV}$ ) has never been explained since the 1960's.

A four-body coupled-channel calculation was carried out with separable potentials of central nature [96]. Recently, Hiyama et al. developed the four-body calculation taking into account both the $\Lambda \mathrm{N}-\Sigma \mathrm{N}$ coupling effect and the Coulomb potentials between charged particles (p, $\left.\Sigma^{ \pm}\right)$, and compared with the experimental results for ${ }_{\Lambda}^{4} \mathrm{He}$ and ${ }_{\Lambda}^{4} \mathrm{H}$ as shown in Fig. 5.7

However, the calculated ground state energy difference between ${ }_{\Lambda}^{4} \mathrm{He}$ and ${ }_{\Lambda}^{4} \mathrm{H}$ was obtained to be $\Delta E_{\text {Cal. }}=2.28-2.33=-0.05 \mathrm{MeV}$ [97] and was inconsistent with the experimental result. 


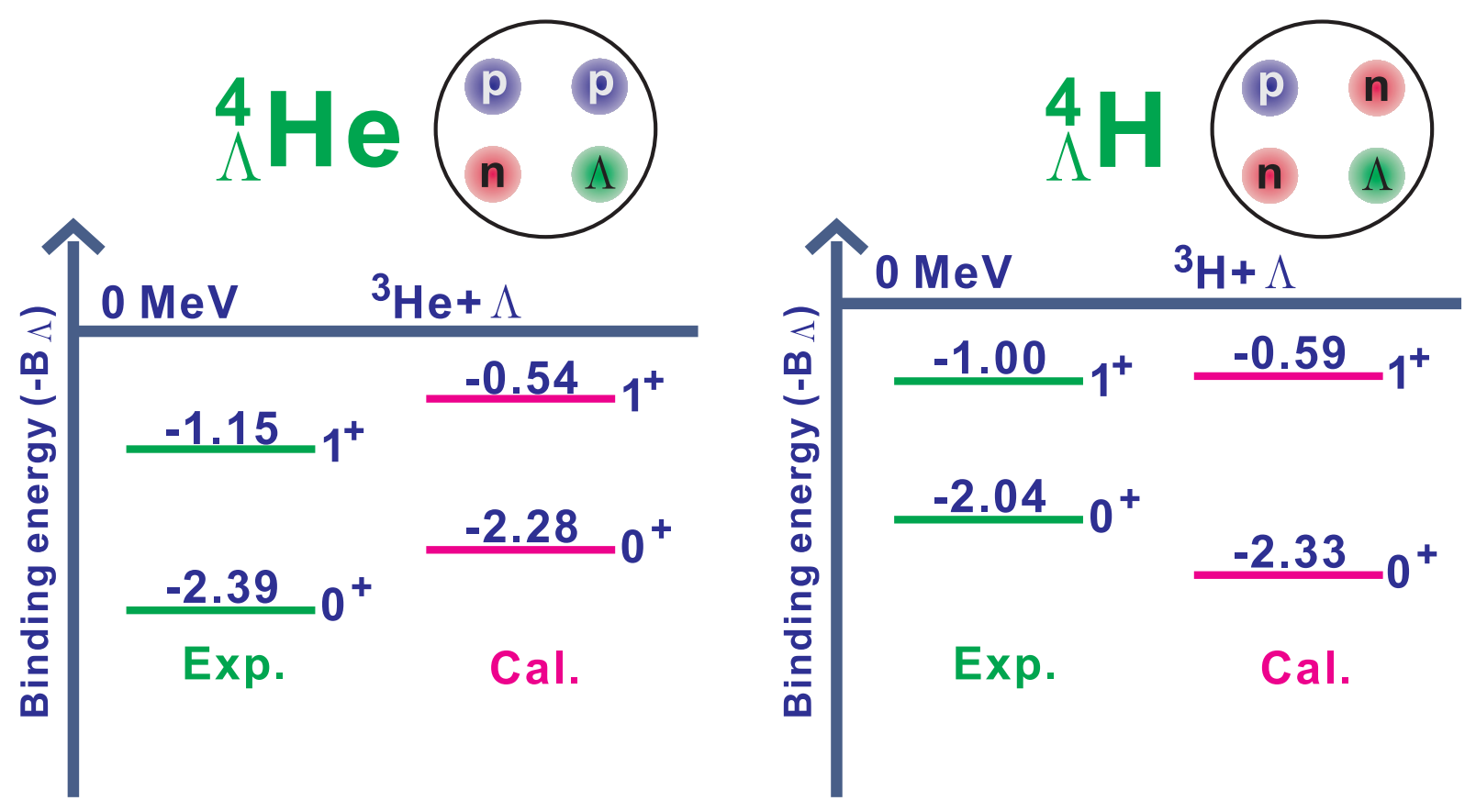

Figure 5.7: Calculated and measured binding energies comparison for ${ }_{\Lambda}^{4} \mathrm{H}$ and ${ }_{\Lambda}^{4} \mathrm{He}$ [97].

Later, Nogga estimated the ${ }_{\Lambda}^{4} \mathrm{H}$ and ${ }_{\Lambda}^{4} \mathrm{He}$ binding energy difference $\left(\Delta E_{\text {Cal. }}=1.54-1.47=0.07\right.$ $\mathrm{MeV}$ ) taking into account the $\Sigma \mathrm{N}-\Lambda \mathrm{N}$ coupling for $\Sigma^{+}, \Sigma^{0}$ and $\Sigma^{-}$with different masses, which was found to be too small if the realistic YN interaction (NSC97) is used [98].

Although there may exist an ambiguity in the experimental data due to systematic errors, the inconsistency between $\Delta E_{\text {Exp. }}$ and $\Delta E_{\text {Cal. }}$ is considered to be due to a large charge symmetry breaking in the $\Lambda \mathrm{N}$ interaction, which is not yet understood.

Since the discussion on the charge symmetry breaking effect has been done using only the $A=4$ $\Lambda$ hypernuclear data so far, this subject should be investigated with other $\Lambda$ hypernuclei.

The energy levels of the low-lying $A=7 ; T=1$ isospin-triplet $\Lambda$ hypernuclei by the fourbody calculation [99] are shown and compared with the observed data of ${ }_{\Lambda}^{7} \mathrm{Be}$ and ${ }_{\Lambda}^{7} \mathrm{Li}$ [57] in Fig. 5.8. All the energy levels in Fig. 5.8 are re-scaled from the $\alpha+\Lambda+N+N$ threshold for each of three $\Lambda$ hypernuclei. The experimental data of ${ }_{\Lambda}^{7} \mathrm{Li}$ energy was obtained using the measured $3877 \pm 5 \pm 7 \mathrm{keV} \gamma$-ray energy for the $M 1\left(1 / 2^{+}(T=1) \rightarrow 1 / 2^{+}(T=0)\right)$ transition in ${ }_{\Lambda}^{7} \mathrm{Li}$ [100] and the ${ }_{\Lambda}^{7} \mathrm{Li}$ ground state binding energy from emulsion data [57].

Though the calculation reproduces the experimental energy level of ${ }_{\Lambda}^{7} \mathrm{Li}\left(T=1,1 / 2^{+}\right)$within $0.08 \mathrm{MeV}$ accuracy, it does not reproduce that of ${ }_{\Lambda}^{7} \mathrm{Be}$ at all.

Therefore, the precise experimental data of ${ }_{\Lambda}^{7} \mathrm{He}$ binding energy is required to verify the charge symmetry breaking effect and to understand the $\Lambda \mathrm{N}$ interaction.

\section{Experimental result}

The measured ${ }_{\Lambda}^{7}$ He hypernuclear mass spectrum using the $\left(\mathrm{e}, \mathrm{e}^{\prime} \mathrm{K}^{+}\right)$reaction is given in Fig. 5.9 (b) and the obtained binding energy and the cross section for the prominent peak (\#1) are shown in Table 5.8. The prominent peak, \#1, is interpreted as a proton-hole state with a $\Lambda$ hyperon in the configuration of $\left[{ }^{6} \mathrm{He}\left(0^{+} ; 0.00\right) \otimes s_{1 / 2 \Lambda}\right]$.

The spectrum is fitted with a Gaussian, and the peak width obtained is $0.73 \pm 0.16 \mathrm{MeV}$ (FWHM), which gives the energy resolution. 


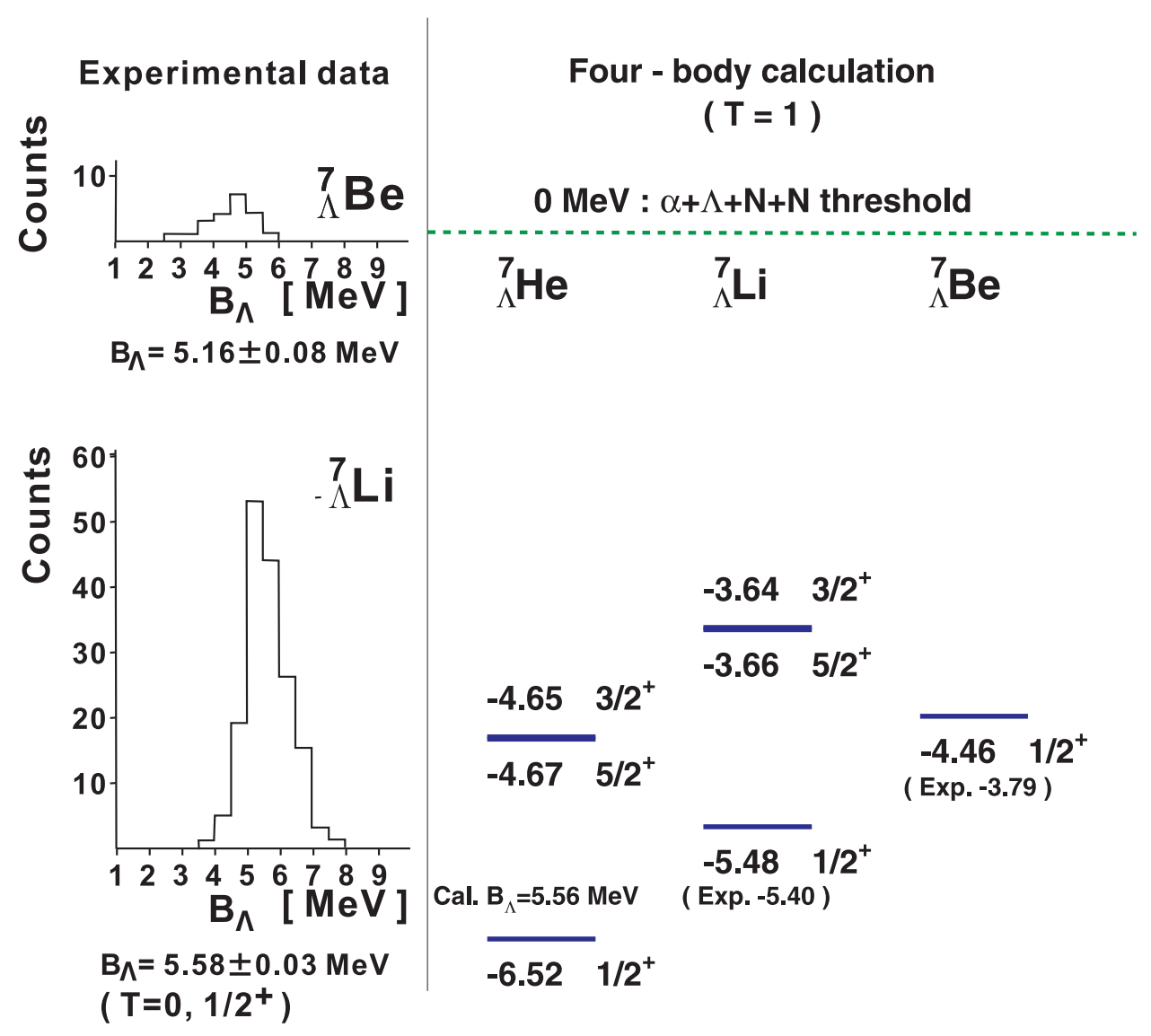

Figure 5.8: Calculated energies of the low-lying states of $A=7 ; T=1$ isospin-triplet $\Lambda$ hypernuclei [99] and measured binding energies of ${ }_{\Lambda}^{7} \mathrm{Be}$ and ${ }_{\Lambda}^{7} \mathrm{Li}[57]$. Energies are re-scaled from the $\alpha+\Lambda+\mathrm{N}+\mathrm{N}$ threshold. 
Table 5.8: Results of fitting the ${ }_{\Lambda}^{7}$ He spectrum.

\begin{tabular}{c|c|c|c}
\hline \hline Peak & $-B_{\Lambda}[\mathrm{MeV}]$ & FWHM $[\mathrm{MeV}]$ & $\left.\overline{(d \sigma / d \Omega)}\right|_{1^{\circ}-13^{\circ}[\mathrm{nb} / \mathrm{sr}]}$ \\
No. & $\pm($ statistic $) \pm($ systematic $)$ & $\pm($ statistic $)$ & $\pm($ statistic $) \pm($ systematic $)$ \\
\hline$\# 1$ & $-5.1 \pm 0.2 \pm 0.1$ & $0.73 \pm 0.16$ & $12 \pm 3 \pm 3$ \\
\hline \hline
\end{tabular}

Several emulsion experiments tried to measure the binding energy of the ${ }_{\Lambda}^{7}$ He ground state in the 1970's [57], using two decay modes :

$$
\begin{aligned}
& { }_{\Lambda}^{7} \mathrm{He} \rightarrow \pi^{-}+{ }^{3} \mathrm{H}+{ }^{4} \mathrm{He}, \\
& { }_{\Lambda}^{7} \mathrm{He} \rightarrow \pi^{-}+{ }^{1} \mathrm{H}+{ }^{3} \mathrm{H}+{ }^{3} \mathrm{H} .
\end{aligned}
$$

However, it has been difficult to determine the binding energy due to the severe background and low statistics.

The binding energy of the ${ }_{\Lambda}^{7}$ He ground state obtained by the present study for the first time is $5.1 \pm 0.2$ (stat.) \pm 0.1 (syst.) $\mathrm{MeV}$.

\section{Comparison of the ${ }_{\Lambda}^{7} \mathrm{He}$ and ${ }_{\Lambda}^{7} \mathrm{Li}$ spectra}

The observed ${ }_{\Lambda}^{7}$ He hypernuclear spectrum can be compared with the ${ }_{\Lambda}^{7} \mathrm{Li}$ spectrum measured with the $\left(\pi^{+}, \mathrm{K}^{+}\right)$reaction. The ${ }_{\Lambda}^{7} \mathrm{Li}$ spectrum was measured by the SKS with $p_{\pi^{+}}=1.05$ $\mathrm{GeV} / c$ of $\pi^{+}$beam at the K6 beamline of the KEK $12 \mathrm{GeV}$ PS in 1998 (KEK-E336) [30], as shown in Fig. 5.9 (a). The measured binding, excitation energies and cross sections are listed in Table 5.9.

Table 5.9: Results of fitting the ${ }_{\Lambda}^{7} \mathrm{Li}$ spectrum taken by the $\left(\pi^{+}, \mathrm{K}^{+}\right)$reaction at KEK [30, 100]. Here, $E_{x}$ shows the excitation energy. Calculated cross sections integrated over $\theta_{\mathrm{K}^{+}}=0^{\circ}-15^{\circ}$ [101] are also compared with the experimental data.

\begin{tabular}{ccc|c|cc}
\hline \hline $\begin{array}{c}\text { Peak } \\
\text { No. }\end{array}$ & $B_{\Lambda}$ or $E_{x}$ & FWHM & $J^{\pi}(T)$ & \multicolumn{2}{c}{ Cross sections $[\mu \mathrm{b} / \mathrm{sr}]$} \\
& {$[\mathrm{MeV}]$} & {$[\mathrm{MeV}]$} & & $\sigma_{2^{\circ}-14^{\circ}(\text { Exp.) }}$ & $\sigma_{0^{\circ}-15^{\circ} \text { (Cal.) }}$ \\
\hline$\# 1$ & $B_{\Lambda}=5.22 \pm 0.08$ & 1.81 (fixed) & $1 / 2^{+}(T=0)$ & $0.345 \pm 0.032$ & 1.21 \\
& & & $3 / 2^{+}(T=0)$ & & 0.13 \\
$\# 2$ & $E_{x}=2.05$ (fixed) & 1.81 (fixed) & $5 / 2^{+}(T=0)$ & $0.486 \pm 0.032$ & 1.23 \\
& & & $7 / 2^{+}(T=0)$ & & 0.08 \\
$\# 3$ & $E_{x}=3.88$ (fixed) & 1.81 (fixed) & $1 / 2^{+}(T=1)$ & $0.241 \pm 0.033$ & 0.60 \\
$\# 4$ & $E_{x}=5.61 \pm 0.24$ & 1.81 (fixed) & $5 / 2^{+}(T=1)$ & $0.136 \pm 0.050$ & $\mathrm{~N} / \mathrm{A}$ \\
& & & $3 / 2^{+}(T=1)$ & & $\mathrm{N} / \mathrm{A}$ \\
$\# 5$ & $E_{x}=7.99 \pm 0.37$ & $3.81 \pm 0.81$ & $3 / 2^{-}(T=0)$ & $0.398 \pm 0.078$ & $\mathrm{~N} / \mathrm{A}$ \\
\hline \hline
\end{tabular}

The precise level scheme of ${ }_{\Lambda}^{7} \mathrm{Li}$ is shown in Fig. 5.9 (b) with measured energies of $\gamma$-ray 
transitions [100].

The bound state region of the observed ${ }_{\Lambda}^{7} \mathrm{Li}$ spectrum was decomposed into four peaks (\#1 $\sim \# 4$ in Fig. 5.9 (a) and (b)) [30], the energies and relative intensities of which agreed fairly well with theoretically calculated level energies and cross sections [101,102] of the four most strongly populated states, namely, $1 / 2^{+}(T=0), 5 / 2^{+}(T=0), 1 / 2^{+}(T=1), 5 / 2^{+}(T=1)$, respectively. The $1 / 2^{+}(T=1)$ state, which was observed at $3.88 \mathrm{MeV}$ apart from the $1 / 2^{+}$ $(T=0)$ by an $M 1$ transition in Fig. 5.9 (b), can be compared to the peak marked as \#1 of the ${ }_{\Lambda}^{7}$ He mass spectrum in Fig. 5.9 (c).

Though some intensity for ${ }_{\Lambda}^{7} \mathrm{He}$ excited states such as the $3 / 2^{+}$and $5 / 2^{+}$states is expected to be observed, it is difficult to identify them in the present spectrum due to the limited quality. 

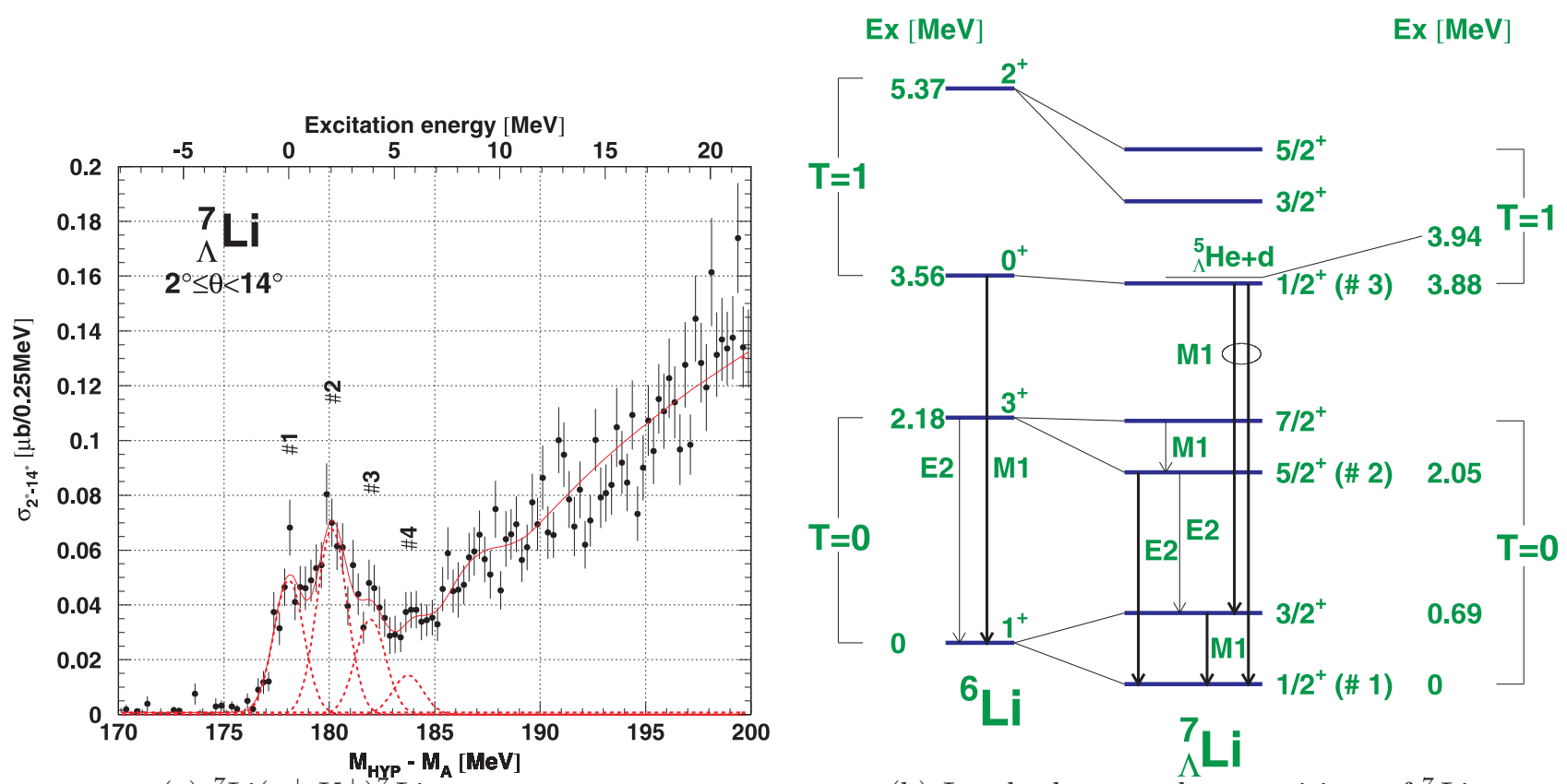

(a) ${ }^{7} \mathrm{Li}\left(\pi^{+}, \mathrm{K}^{+}\right)_{\Lambda}^{\mathbf{H P}} \mathrm{Li}$ mass spectra.

(b) Level scheme and $\gamma$ transitions of ${ }_{\Lambda}^{7} \mathrm{Li}$.

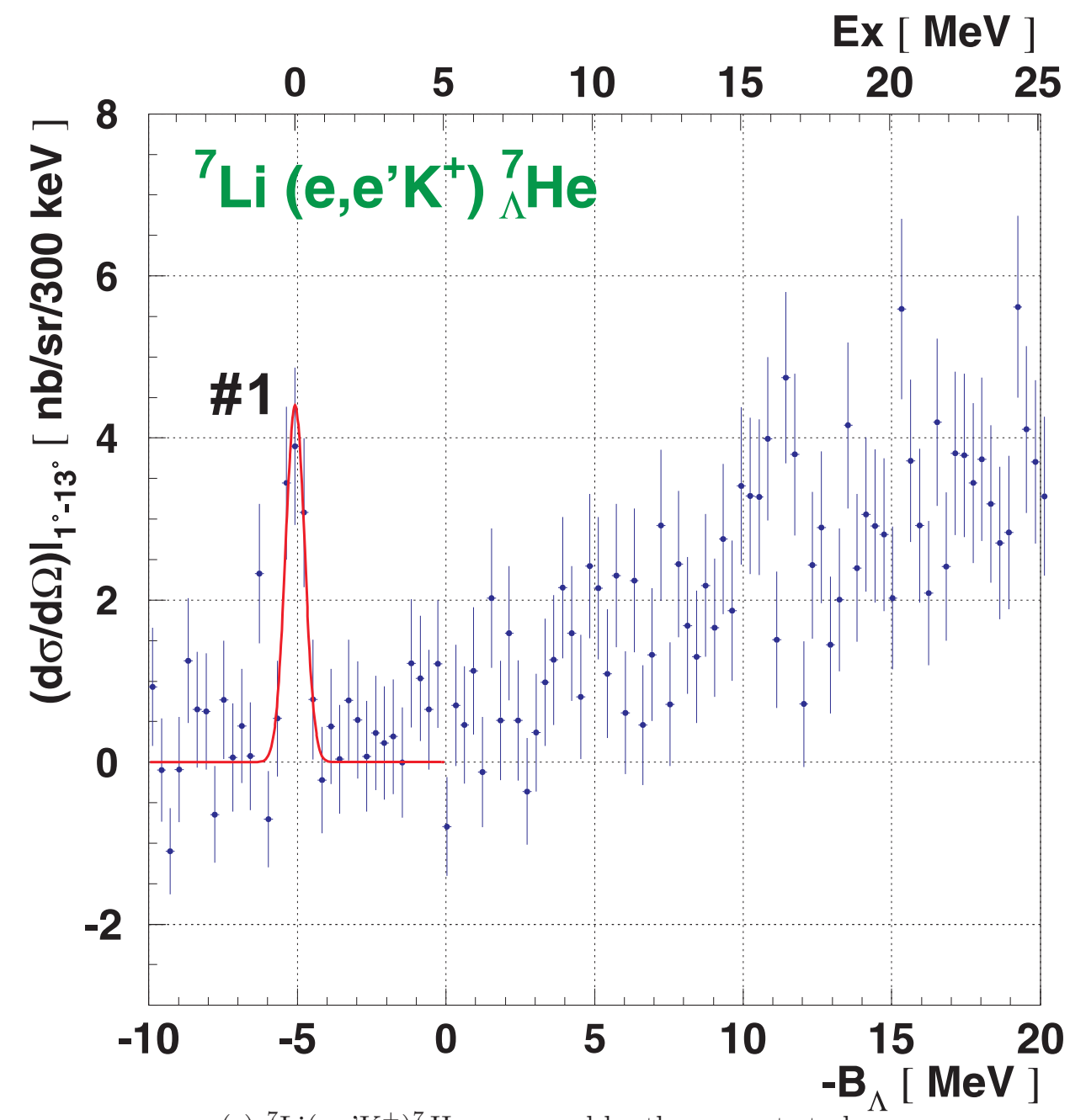

(c) ${ }^{7} \mathrm{Li}\left(\mathrm{e}, \mathrm{e}^{\prime} \mathrm{K}^{+}\right){ }_{\Lambda}^{7} \mathrm{He}$ measured by the present study.

Figure 5.9: (a) Hypernuclear mass spectra for ${ }_{\Lambda}^{7} \mathrm{Li}$ with the $\left(\pi^{+}, \mathrm{K}^{+}\right)$reaction measured by KEK-SKS E336 [30], (b) level scheme and $\gamma$ transitions of ${ }_{\Lambda}^{7} \mathrm{Li}[100]$ and (c) ${ }_{\Lambda}^{7} \mathrm{He}$ with the $\left(\mathrm{e}, \mathrm{e}^{\prime} \mathrm{K}^{+}\right)$reaction around the bound region. 


\section{Theoretical calculations of the ${ }_{\Lambda}^{7} \mathrm{He}$ excitation spectrum}

The ${ }_{\Lambda}^{7}$ He hypernucleus has been investigated theoretically within the frame work of both cluster and shell models.

In this section, the experimental results of the binding energies are discussed referring to the cluster model, and the cross sections are compared with the shell model calculation.

\section{Shell model}

The level structure and decay properties of ${ }_{\Lambda}^{6} \mathrm{Li}$ has been investigated with the translationally invariant shell model (TISM) by Majling et al. [103] and well reproduce experimental data [19$22]$ by the $\left(\mathrm{K}^{-}, \pi^{-}\right)$reaction.

This framework was extended to the $A=7$ system by Richter, Sotona and Žofka [104] by modifying the model for ${ }_{\Lambda}^{6} \mathrm{Li}$.

The transition matrix of the electro-production of hypernuclei is described with leptonic and hadronic parts independently as discussed in Eqn. 1.19 (see Section 1.5).

There are three approaches ;

1) chiral-quark, color-dielectric models,

2) models with quarks and mesons, i.e. effective theory of QCD,

3) isobar models with effective theory of QHD,

for the treatment of the direct term in the hadronic part for the reaction with virtual photon energies up to $E_{\gamma}^{\mathrm{Lab}}=2.5 \mathrm{GeV}$.

Sotona and Bydzovsky analyzed and compared ${ }_{\Lambda}^{7}$ He hypernuclear properties with a formalism of DWIA adopting three modern isobar models denoted hereafter as Saclay-Lyon (SLA) [90], Williams-Ji-Cotanch (C4) [91], and Kaon-MAID (KMAID) [92]. The differences between these isobar model are mainly the set of baryon resonances included, whether the hadronic form factor is involved or not, and whether the $\mathrm{SU}(3)$ symmetry constraint is required or not.

The experimental results and theoretical prediction [85] of the ${ }^{7} \mathrm{Li}\left(\mathrm{e}, \mathrm{e}^{\prime} \mathrm{K}^{+}\right){ }_{\Lambda}^{7} \mathrm{He}$ cross sections and excitation energies are compared in Table 5.10. The beam energy, $E_{\text {beam }}=1.8 \mathrm{GeV}$, and kaon scattering angle of $1^{\circ}<\theta_{\mathrm{K}^{+}}^{\mathrm{Lab}}<13^{\circ}$ are integrated for each theoretical calculation.

Table 5.10: Comparison of experimental results of ${ }^{7} \mathrm{Li}\left(\mathrm{e}, \mathrm{e}^{\prime} \mathrm{K}^{+}\right){ }_{\Lambda}^{7} \mathrm{He}$ cross section for the ground state with theoretical predictions [85].

\begin{tabular}{|c|c|c|c|c|c|c|c|}
\hline & \multicolumn{2}{|c|}{ Experimental results } & \multicolumn{5}{|c|}{ Theoretical prediction } \\
\hline \multirow{2}{*}{$\begin{array}{c}\text { peak } \\
\text { no. }\end{array}$} & \multirow{2}{*}{$\begin{array}{c}\mathrm{Ex} \\
{[\mathrm{MeV}]}\end{array}$} & \multirow{2}{*}{$\begin{array}{c}\left.\overline{(d \sigma / d \Omega)}\right|_{1^{\circ}-13^{\circ}} \\
{[\mathrm{nb} / \mathrm{sr}]}\end{array}$} & \multirow[t]{2}{*}{$J^{\pi}$} & \multirow{2}{*}{$\begin{array}{c}\mathrm{Ex} \\
{[\mathrm{MeV}]}\end{array}$} & \multicolumn{3}{|c|}{$\left.\overline{\overline{(d \sigma / d \Omega)}}\right|_{1^{\circ}-13^{\circ}[\mathrm{nb} / \mathrm{sr}]}$} \\
\hline & & & & & SLA & $\mathrm{C} 4$ & KMAID \\
\hline \multirow[t]{3}{*}{$\# 1$} & 0.0 & $12 \pm 3 \pm 3$ & $1 / 2^{+}$ & 0 & 13.2 & 16.2 & 9.7 \\
\hline & & & $5 / 2^{+}$ & 1.59 & 3.0 & 3.7 & 2.5 \\
\hline & & & $3 / 2^{+}$ & 1.94 & 2.3 & 2.7 & 1.5 \\
\hline
\end{tabular}

Each theoretical prediction of the ${ }_{\Lambda}^{7}$ He ground state cross section reproduces the experimental result within the error. 

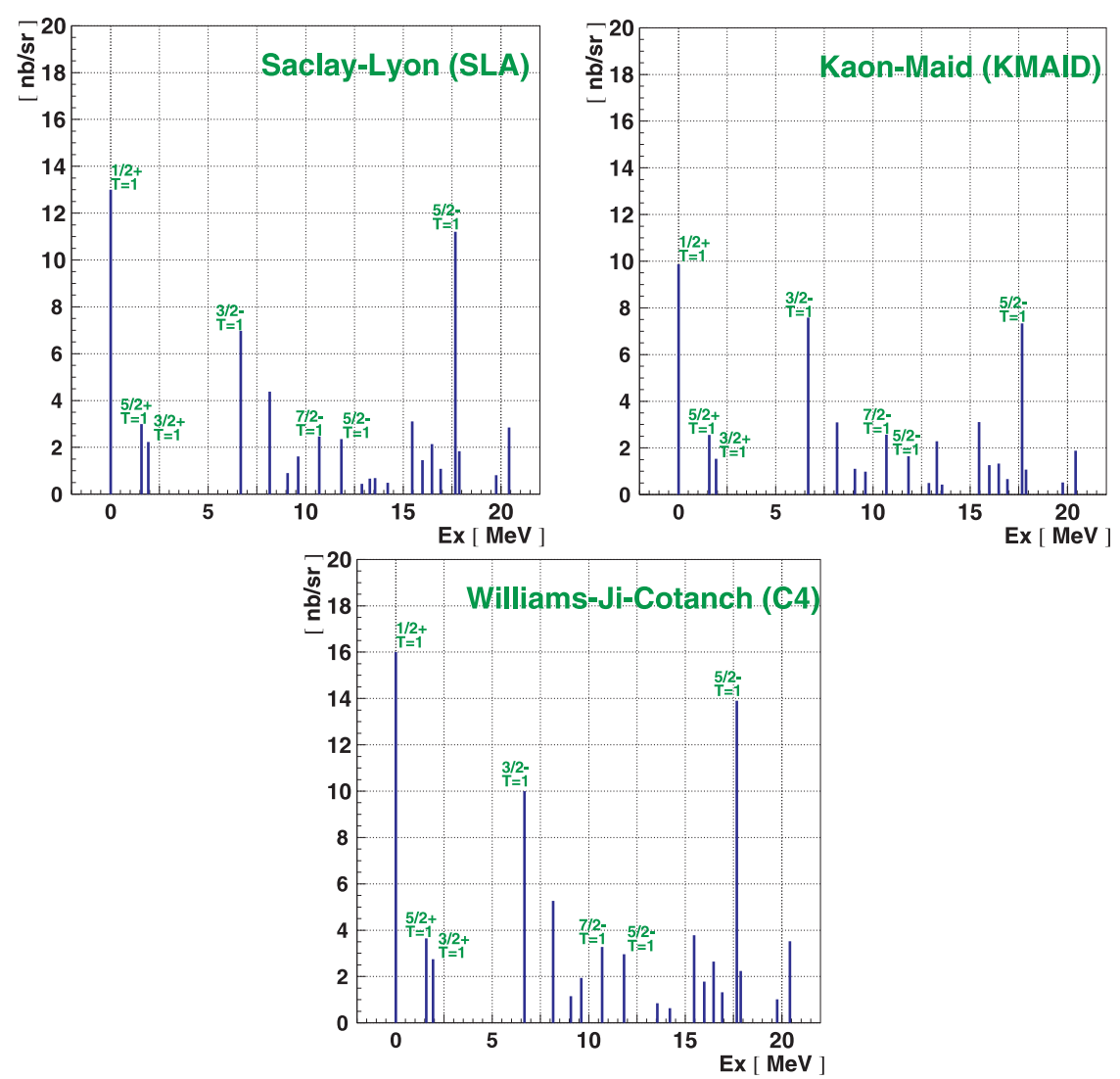

Figure 5.10: Theoretical calculations of the ${ }_{\Lambda}^{7} \mathrm{He}$ cross sections and excitation energies with three types of isobar models, Saclay-Lyon (left), Kaon-MAID (center) and Williams-Ji-Cotanch (right) models. 


\section{Cluster model}

The cluster model with four-body calculation has been developed by Hiyama et al. [97, 99]. This model was modified from the three-body cluster calculation which well reproduced observed binding energies of the ground states of ${ }_{\Lambda}^{6} \mathrm{He}$ and ${ }_{\Lambda}^{6} \mathrm{Li}[105]$.

Applying this method, $A=7$ hypernuclear triplets $\left({ }_{\Lambda}^{7} \mathrm{He},{ }_{\Lambda}^{7} \mathrm{Li}\right.$ and $\left.{ }_{\Lambda}^{7} \mathrm{Be}\right)$ can be described as $(\alpha+\mathrm{N}+\mathrm{N}+\Lambda)$ as shown in Fig. 5.11.

\section{$A=7$ iso-triplet ( four-body system )}
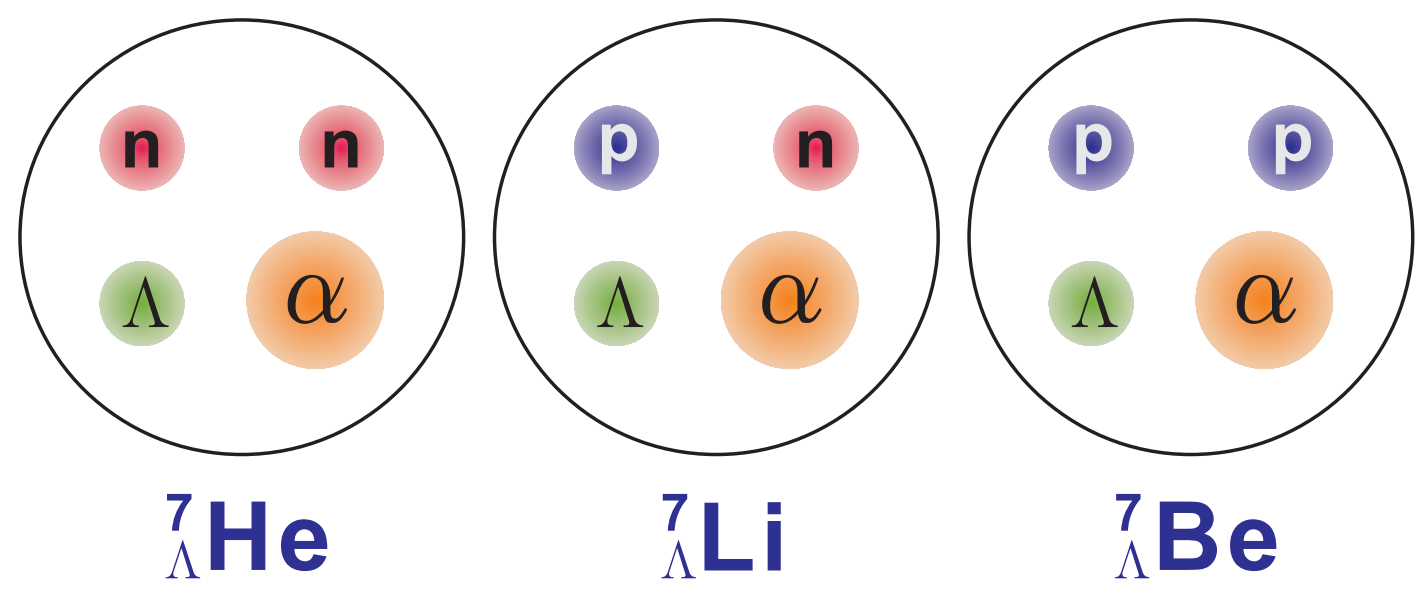

Figure 5.11: Models of the $A=7$ triplets with the four-body cluster calculation.

The four-body calculation adopts the Nijmegen model (NSC97f) interaction, in which the $\Sigma \mathrm{N}$ channel contribution is renormalized into $\Lambda \mathrm{N}$ interactions, and the strength of the $\Lambda \mathrm{N}$ spin-spin interaction was adjusted to reproduce the splitting of the $0^{+}$and $1^{+}$states of ${ }_{\Lambda}^{4} \mathrm{H}[106]$.

The predicted $\Lambda$ binding energy of the ${ }_{\Lambda}^{7}$ He ground state $\left(1 / 2^{+}\right)$is compared with that of our experimental result in Table 5.11.

Table 5.11: Comparison of experimental result of the ${ }_{\Lambda}^{7}$ He ground state binding energy with theoretical prediction by Hiyama et al.[99].

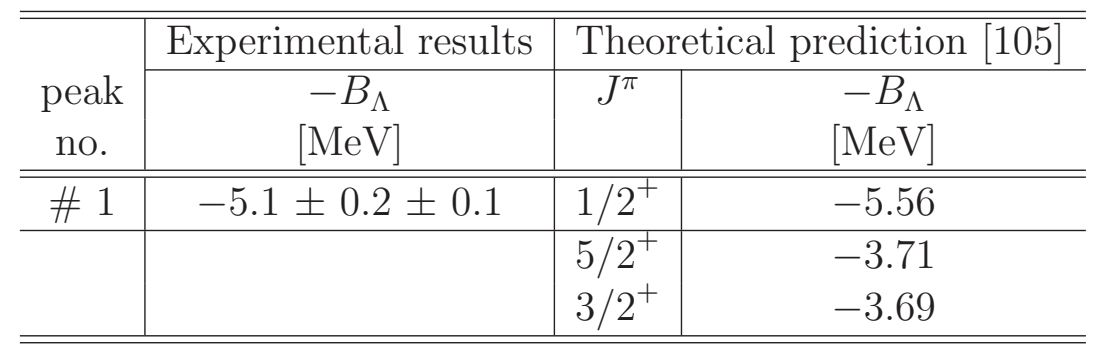

The calculated energy spectrum of low-lying states of ${ }_{\Lambda}^{7} \mathrm{He}$ is shown in Fig. 5.12 (b) in comparison to the measured spectrum of ${ }^{6} \mathrm{He}$ nucleus [99]. The low-lying ${ }_{\Lambda}^{7}$ He states are interpreted as the configurations of ${ }^{6} \mathrm{He}$ ground state $\left(0^{+}\right)$with $s_{1 / 2 \Lambda}$ for $1 / 2^{+}$state and of spin-doublet of 


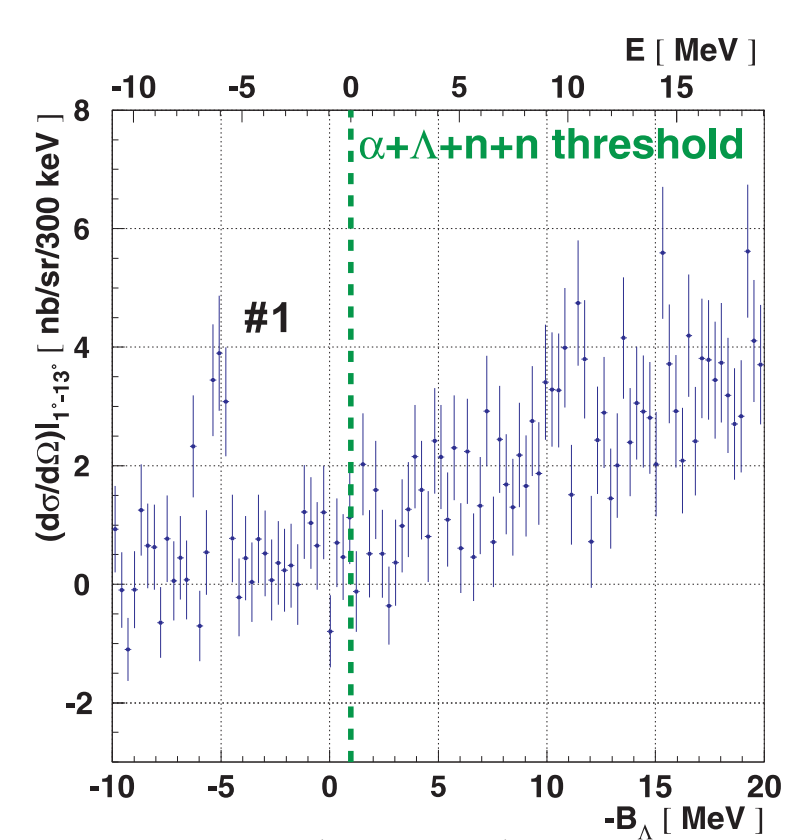

(a) The observed ${ }^{7} \mathrm{Li}\left(\mathrm{e}, \mathrm{e}^{\prime}, \mathrm{K}^{+}\right){ }_{\Lambda}^{7} \mathrm{He}$ mass spectra with re-scale.

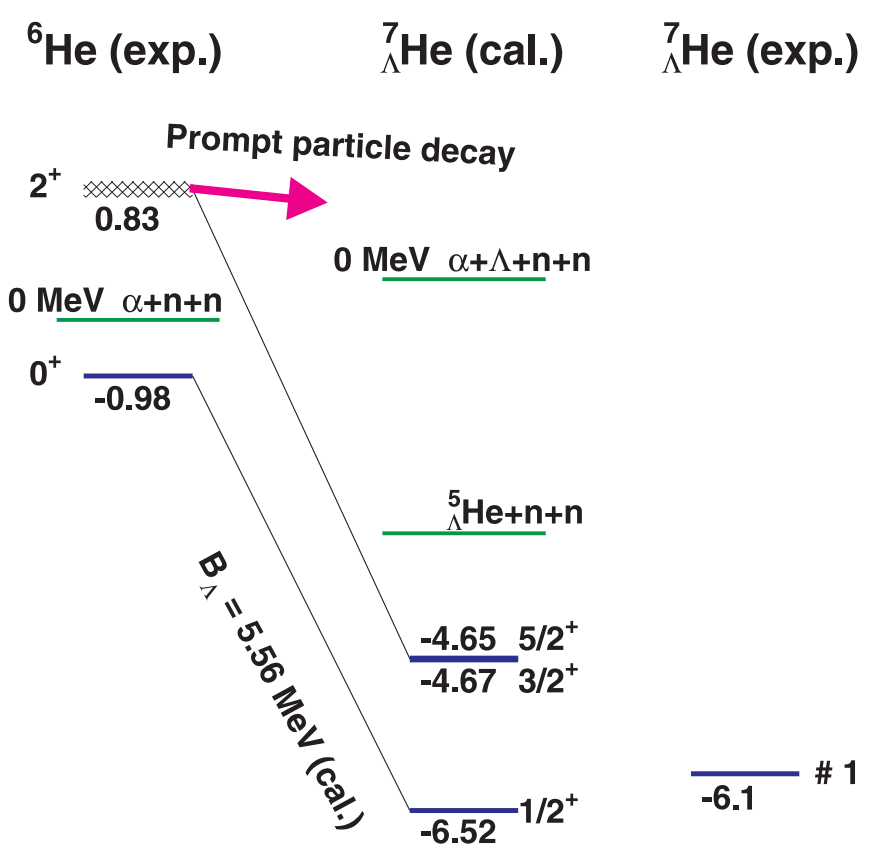

(b) Calculated and measured energy levels comparison for ${ }^{6} \mathrm{He}$ and ${ }_{\Lambda}^{7} \mathrm{He}$.

Figure 5.12: The observed ${ }^{7} \mathrm{Li}\left(\mathrm{e}, \mathrm{e}^{\prime}, \mathrm{K}^{+}\right){ }_{\Lambda}^{7} \mathrm{He}$ mass spectrum re-scaled with $E=0 \mathrm{MeV}$ at the $\alpha+\Lambda+$ $\mathrm{n}+\mathrm{n}$ four body threshold (a) and calculated energy spectra of the low-lying states of ${ }_{\Lambda}^{7} \mathrm{He}(\mathrm{b})$. The re-scaled energy scheme of the observed peak (\# 1) is shown for comparison.

${ }^{6} \mathrm{He}$ excited state $\left(2^{+}\right)$with $s_{1 / 2 \Lambda}$ for $3 / 2^{+}$and $5 / 2^{+}$states.

The two states, $3 / 2^{+}$and $5 / 2^{+}$, of ${ }_{\Lambda}^{7} \mathrm{He}$, which are composed of the $2^{+}$unbound state of ${ }^{6} \mathrm{He}$, are expected to decay with $\tau \sim 10^{-10}$ sec. The energy spacing between $3 / 2^{+}$and $5 / 2^{+}$are estimated to be $\sim 20$ or $\sim 500 \mathrm{keV}$ by the three or four body calculation, respectively.

\section{Discussion of the charge symmetry of the $A=7$ isospin-triplet}

Discussion of the charge symmetry of the $A=7$ isospin-triplet has become possible for the first time by the present study.

The energy level schemes of the $A=7 ; T=1$ isospin-triplet $\Lambda$ hypernuclei, ${ }_{\Lambda}^{7} \mathrm{He},{ }_{\Lambda}^{7} \mathrm{Li}$ and ${ }_{\Lambda}^{7} \mathrm{Be}$, by the cluster-model calculation [99] are presented and compared with the observed energies by the emulsion experiments [57] for ${ }_{\Lambda}^{7} \mathrm{Li}$ and ${ }_{\Lambda}^{7} \mathrm{Be}$ and by the present study for ${ }_{\Lambda}^{7} \mathrm{He}$ in Fig. 5.13. All the energies in Fig. 5.13 are re-scaled from the $\alpha+\Lambda+\mathrm{N}+\mathrm{N}$ threshold of each $\Lambda$ hypernucleus both for measured and calculated data.

The four-body cluster-model calculation involves the $\Lambda \mathrm{N}-\Sigma \mathrm{N}$ coupling effect in the $\Lambda \mathrm{N}$ interaction and the Coulomb potentials between charged particles $\left((\mathrm{p},) \Sigma^{ \pm}\right)$are included. Comparing absolute energy differences $(\Delta E)$ between measured and calculated energy of each $\Lambda$ hypernuclear ground state $\left(1 / 2^{+}, T=1\right)$, the calculation reproduces the ${ }_{\Lambda}^{7} \mathrm{Li}$ ground state energy within $0.1 \pm 0.03 \mathrm{MeV}(1.8 \%)$. However, the calculated results of ground state energy for ${ }_{\Lambda}^{7} \mathrm{He}$ and ${ }_{\Lambda}^{7} \mathrm{Be}$ are inconsistent by $0.4 \pm 0.2 \mathrm{MeV}(6.6 \%)$ and $0.7 \pm 0.08 \mathrm{MeV}(18.5 \%)$ in the energy difference, respectively. The inconsistency of these energy differences cannot be explained with the $\Lambda \mathrm{N}-\Sigma \mathrm{N}$ coupling effect or Coulomb effect, and seems to suggest the existence of charge symmetry breaking in the $\Lambda \mathrm{N}$ interaction. 


\section{$A=7$ isospin-triplet level scheme $0 \mathrm{MeV}: \alpha+\Lambda+\mathrm{N}+\mathrm{N}$ threshold

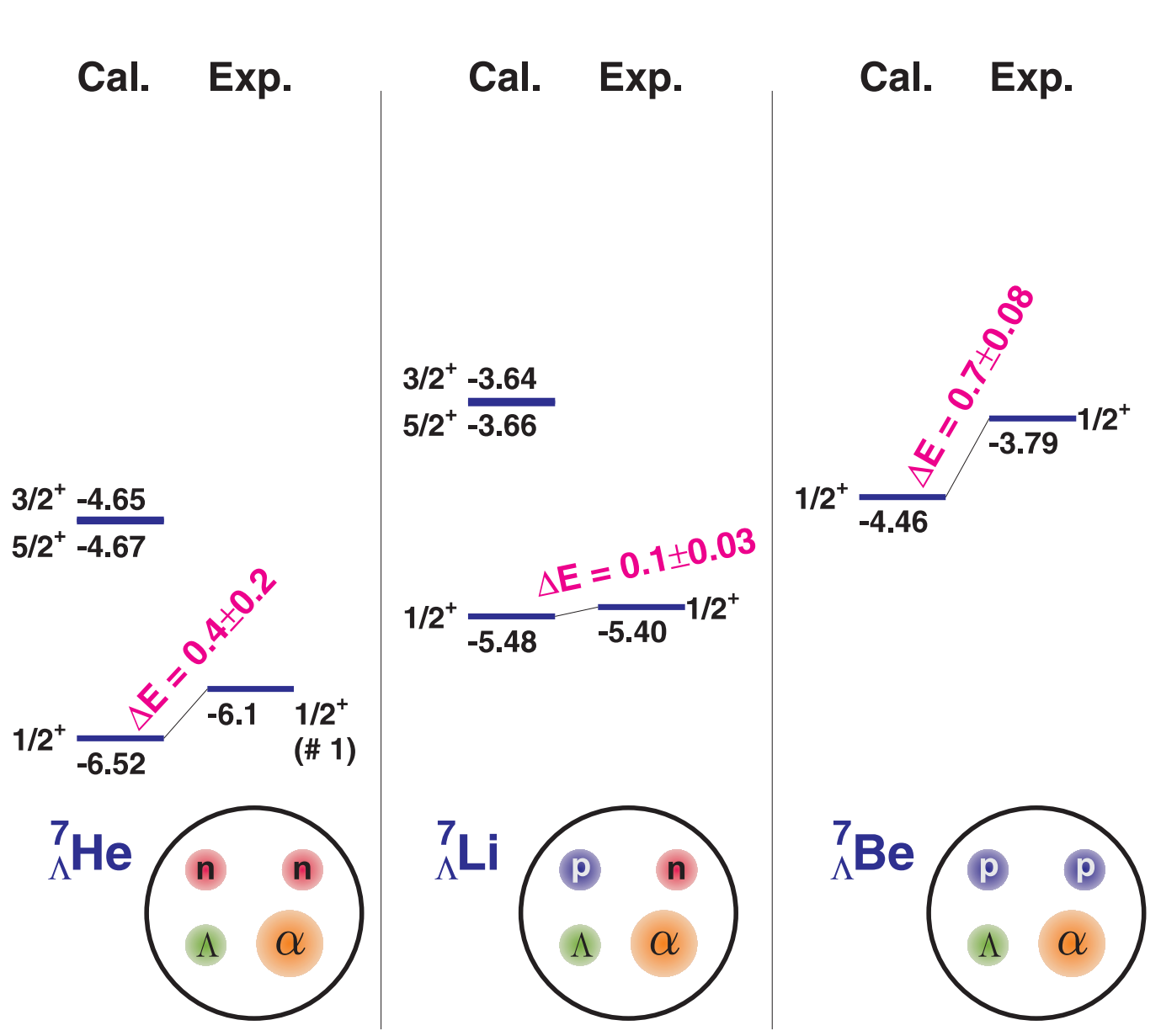

Figure 5.13: Comparison between theoretical and experimental energy scheme for the $A=7$ isospintriplet $\Lambda$ hypernuclei. All the energy levels are re-scaled from the $\alpha+\Lambda+\mathrm{N}+\mathrm{N}$ threshold for each of three $\Lambda$ hypernucleus. The errors are statistical only. 


\section{Chapter 6}

\section{Conclusion}

Hypernuclear production through the $\left(\mathrm{e}, \mathrm{e}^{\prime} \mathrm{K}^{+}\right)$reaction achieves high resolution spectroscopy thanks to the high quality and continuous primary electron beam. It is in contrast to the $\left(\mathrm{K}^{-}, \pi^{-}\right)$and $\left(\pi^{+}, \mathrm{K}^{+}\right)$reactions, although the hypernuclear cross sections for the $\left(\mathrm{e}, \mathrm{e}^{\prime} \mathrm{K}^{+}\right)$reaction are much smaller.

A goal of the present study is to further explore and establish $\left(\mathrm{e}, \mathrm{e}^{\prime} \mathrm{K}^{+}\right)$spectroscopy as a tool to investigate $\Lambda$ hypernuclei, succeeding the pioneering experiment JLab E89-009, which was successfully carried out previously.

The $1.8 \mathrm{GeV}$ electron beam hit targets at the entrance of the splitter magnet in JLab Hall C. Positive kaons and scattered electrons were deflected to the opposite directions and guided to the high resolution kaon spectrometer (HKS) and the scattered electron spectrometer (Enge). The HKS, which has a high momentum resolution $\left(\Delta p / p \sim 2.0 \times 10^{-4}\right.$ [FWHM] $)$ and a large solid angle (16 msr with the splitter), was newly constructed for (e, $\left.\mathrm{e}^{\prime} \mathrm{K}^{+}\right)$hypernuclear spectroscopy. The Enge, which was also used in E89-009, was tilted vertically by 8 degrees perpendicular to the splitter dispersion plane in order to suppress Bremsstrahlung and Møller electrons that cause huge backgrounds (tilt method). The present experimental configuration allows us to conduct high luminosity hypernuclear experiments with the $\left(\mathrm{e}, \mathrm{e}^{\prime} \mathrm{K}^{+}\right)$reaction.

Data on three targets, $\mathrm{CH}_{2}\left(465 \mathrm{mg} / \mathrm{cm}^{2}\right),{ }^{7} \mathrm{Li}\left(189 \mathrm{mg} / \mathrm{cm}^{2}\right)$ and ${ }^{12} \mathrm{C}\left(100 \mathrm{mg} / \mathrm{cm}^{2}\right)$, were analyzed in the present study. The elementary processes $\mathrm{p}\left(\mathrm{e}, \mathrm{e}^{\prime} \mathrm{K}^{+}\right) \Lambda / \Sigma^{0}$ were used for calibration of the absolute scale of missing mass. The hypernuclear spectra of ${ }_{\Lambda}^{12} \mathrm{~B}$ and ${ }_{\Lambda}^{7} \mathrm{He}$ were also obtained with the best resolution for hypernuclear spectroscopy $\left(490 \pm 70 \mathrm{keV}, \mathrm{FWHM}\right.$ for ${ }_{\Lambda}^{12} \mathrm{~B}$ ground state doublet).

Prominent peaks corresponding to the states with a $\Lambda$ hyperon in the $s$ and $p$ orbits were observed with improved resolution and statistics from the ones observed in E89-009 experiment. The average binding energy of the ${ }_{\Lambda}^{12} \mathrm{~B}$ ground state doublet was determined to be 11.7 \pm 0.1 (stat.) \pm 0.1 (syst.) $\mathrm{MeV}$ which is consistent with $11.52 \pm 0.35 \mathrm{MeV}$ from the previous experiment, JLab E89-009 and with $11.37 \pm 0.06 \mathrm{MeV}$ by the emulsion data.

The count rate of the ${ }_{\Lambda}^{12} \mathrm{~B}$ ground state doublet was $7.0 \times 10^{-4} \mathrm{~Hz}\left(100 \mathrm{mg} / \mathrm{cm}^{2}, I_{e}=20.2 \mu \mathrm{A}\right)$, being much improved from $1.0 \times 10^{-4} \mathrm{~Hz}\left(22 \mathrm{mg} / \mathrm{cm}^{2}, I_{e}=0.66 \mu \mathrm{A}\right)$ in E89-009.

The cross section of the ${ }_{\Lambda}^{12} \mathrm{~B}$ ground state doublet, which was integrated over the $\mathrm{K}^{+}$scattering angle $\left(1^{\circ}<\theta_{\mathrm{K}^{+}}<13^{\circ}\right.$ ), was deduced to be $54 \pm 10$ (stat.) $\pm{ }_{22}^{25}$ (syst.) nb/sr. The theoretical interpretation has also been provided for this result with the DWIA approximation, and gave a result consistent with the experimental result. 
The ground state $\left(s_{\Lambda}\right)$ peak was observed in the ${ }_{\Lambda}^{7}$ He spectrum for the first time. The binding energy of the ${ }_{\Lambda}^{7}$ He ground state obtained was $5.1 \pm 0.2$ (stat.) \pm 0.1 (syst.) $\mathrm{MeV}$. The observed spectrum of the ${ }_{\Lambda}^{7} \mathrm{He}$ was compared to its charge symmetric hypernucleus ${ }_{\Lambda}^{7} \mathrm{Li}$.

The binding energies for the ground state of $A=7$ isospin-triplet have been investigated by the four-body cluster-model. The calculation reproduced only the experimental data of the ${ }_{\Lambda}^{7} \mathrm{Li}$ ground state $(T=1)$. The inconsistency for other two $A=7$ isospin-triplets, ${ }_{\Lambda}^{7} \mathrm{He}$ and ${ }_{\Lambda}^{7} \mathrm{Be}$, indicates results of the charge symmetry breaking effect. The complete set of experimental data for the $A=7$ isospin-triplet binding energies was obtained by the present study. This data set allows further understanding of $\Lambda \mathrm{N}$ interaction.

The cross section of the ${ }_{\Lambda}^{7} \mathrm{He}$ ground state was measured to be $12 \pm 3$ (stat.) \pm 3 (syst.) $\mathrm{nb} / \mathrm{sr}\left(1^{\circ}<\theta_{\mathrm{K}^{+}}<13^{\circ}\right)$ and compared with a shell model based formalism of DWIA adopting four modern isobar models ; Saclay-Lyon (SLA), Williams-Ji-Cotanch (C4) and Kaon-MAID (KMAID) by M. Sotona and P. Bydzovsky.

The calculated cross sections for the ${ }_{\Lambda}^{7}$ He ground state reproduced the experimental result with any three isobar models.

The second generation of the (e,e' $\left.\mathrm{K}^{+}\right)$hypernuclear spectroscopy experiments is proved to be practical and powerful. We have achieved high energy resolution and high yield rate $\Lambda$ hypernuclear spectroscopy by $\left(\mathrm{e}, \mathrm{e}^{\prime} \mathrm{K}^{+}\right)$reaction, by introducing the HKS spectrometer and the new tilt method. A solid basis toward future high precision $\Lambda$ hypernuclear spectroscopy has been established. 


\section{Acknowledgement}

First of all, I would like to express my special thanks to A. Matsumura for his great efforts and contributions to the experiment. He especially worked on the R\&D and operation of the Enge drift chamber before and during the experiment. He also participated the R\&D of the Water Cerenkov counter. During the experiment, he mainly worked on the trigger electronics setups, the DAQ maintenance in addition to the chamber operation. After the experiment, he contributed to the Monte Carlo calculation, the tracking analysis and maintained the analysis engine. Without his support, this thesis would not have been completed. We experienced Hurricane Isabel in September 2003, which resulted in the postponement of the experiment, spent extremely inconvenience days together without electricity. I will not forget his powerful support and the wealth of experience with him for all my life.

D. Kawama, he participated to the experiment since the end of 2004 as a freshman in the graduate course, and worked very hard to proceed the on-line analysis. He always brought a fresh air to the collaboration and brought fresh ideas to the analysis, too. I believe that he established excellent experiences and knowledge through out E01-011, and I hope that he maximize his contributions to the upcoming experiment, E01-115.

I address my thanks to the partnership of L. Tang and L. Yuan for their energetic promotion of the analysis. They gave me a lecture of the optics optimization which should be helpful for my next carrier in the accelerator division at the Spring-8. Especially, L. Yuan helped my inconveniences in the U.S. life.

I would like to appreciate E.V. Hungerford for his greatest efforts to arrange my VISA application. He also gave me worthful lectures and advices on the estimation of the accidental electrons.

I would like to say my special thanks to J. Reinhold. He always continued to bring novel, in one sense, ideas for the analysis of Čerenkov counter and kaon selection during the E01-011 and test experiments in the KEK 12 GeV-PS. Also, I appreciate D. Gaskell, R. Ent, D. Dutta and P. Markowitz who gave me precise introductions about procedures of data taking in the Jlab. Especially, D. Gaskell imparted much useful knowledge of the Hall C DAQ systems details of beam monitor mechanisms.

I would like to show my appreciation for all of hard engineering works and detector support designs by W. Kellner, S. Hickson, M. Lawing, P. Hood, G. Smith, M. Fowler, B. Metzger, P. Medeiros, W. Vulcan, J. Beaufait and E. Anderson. They always accepted and completed our difficult suggestions and requests at anytime. Especially, J. Beaufait and E. Anderson to paid attention to our safety work environment and gave us various useful suggestions. 
I personally express my special thanks to S.A. Wood for his on-time supports for the DAQ and computer trouble shootings. And he also spent his time for all of this thesis correction.

I appreciate T. Miyoshi, L. Cole, V.M. Rodriguez, P. Bartlin, S. Tomislav and A. Daniel for their hard works on the experimental start-up, day and night shifts.

I would like to address my special acknowledgements to E. Hiyama, who gave me detailed lectures about the theoretical interpretations of the few-body systems, and encouraged my hard analysis.

I appreciate to Y. Fujii who supported my thesis work, especially for the Monte Carlo simulation parts.

I am grateful for H. Tamura who gave me worthful advices on the analysis of spectra, error estimations. He also gave me various kinds of beneficial instructions about electronics, techniques of noise-reduction at the AGS-BNL.

Now, I would like to address my special appreciation to S.N. Nakamura. All of my thesis work has been supported by him since 2000. Also, he was the only person who frequently came over to the JLab from Japan with a fresh perspective on problems. He also provided various kinds of suggestions to my analysis from different point of view.

Finally, I would like to express my best acknowledgement to O. Hashimoto who gave me an opportunity to obtain the brilliant experience in my life through experimental physics, and his support of my analysis. 


\section{Appendix A}

\section{Mass references used in spectra calculation}

Mass references used for the present spectra extraction are shown in Table A.1.

Table A.1: Values of particles, target nuclear masses[107].

\begin{tabular}{cr}
\hline \hline Item & Mass $\left[\mathrm{MeV} / \mathrm{c}^{2}\right]$ \\
\hline electron & 0.511 \\
$\mathrm{~K}^{+}$ & 493.677 \\
proton & 938.272 \\
neutron & 939.565 \\
$\Lambda$ & 1115.683 \\
$\Sigma^{0}$ & 1192.642 \\
\hline${ }^{6} \mathrm{He}$ & 5605.537 \\
${ }_{\Lambda}^{7} \mathrm{He}$ threshold & 6721.220 \\
${ }^{7} \mathrm{Li}$ & 6533.834 \\
\hline${ }^{11} \mathrm{~B}$ & 10252.548 \\
${ }_{\Lambda}^{12} \mathrm{~B}$ threshold & 11368.231 \\
${ }^{12} \mathrm{C}$ & 11174.864 \\
\hline${ }^{27} \mathrm{Al}$ & 25126.506 \\
${ }_{\Lambda}^{28} \mathrm{Al}$ threshold & 26242.189 \\
${ }^{28} \mathrm{Si}$ & 26053.194 \\
\hline \hline
\end{tabular}





\section{Appendix B}

\section{S-factors for proton pick-up reactions}

Table B.1: Relative S-factors for proton pickup reactions on ${ }^{7} \mathrm{Li}$

\begin{tabular}{c|c|c|c}
\hline \hline Reaction & $J^{\pi}$ & Ex $[\mathrm{MeV}]$ & S-factor \\
\hline${ }^{7} \mathrm{Li}(\mathrm{t}, \alpha)^{6} \mathrm{He}$ & $0^{+}$ & 0.00 & 1.08 \\
$E_{t}=38[\mathrm{MeV}]$ & & & \\
{$[108]$} & & & \\
\hline${ }^{7} \mathrm{Li}(\mathrm{e}, \mathrm{e} \cdot \mathrm{p}){ }^{6} \mathrm{He}$ & $0^{+}$ & 0.00 & 0.42 \\
$E_{e}=329.7,454.7$ & $2^{+}$ & 1.79 & 0.16 \\
{$[\mathrm{MeV}][109]$} & & & \\
\hline
\end{tabular}

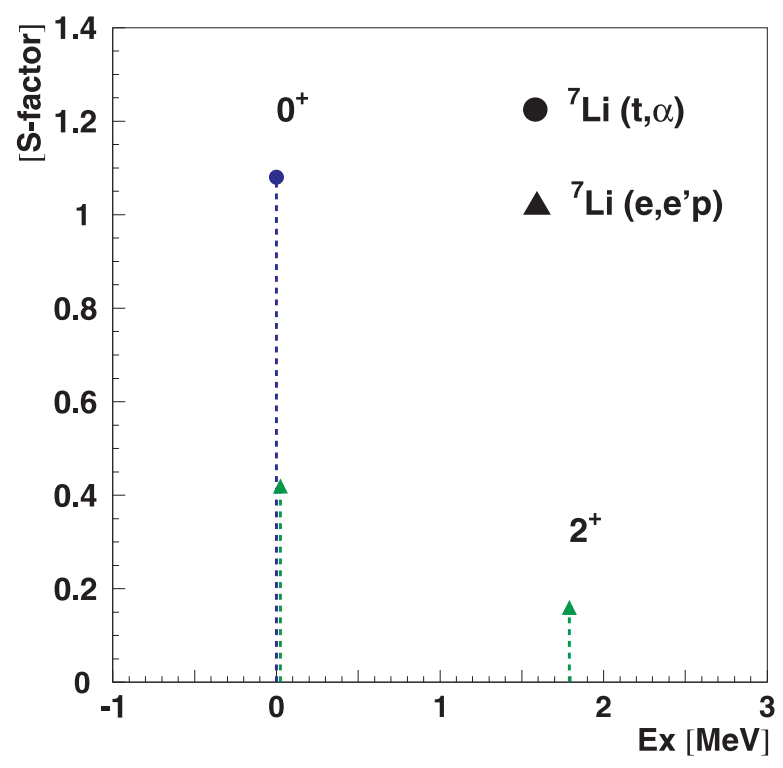

Figure B.1: Relative spectroscopic factors for proton pick-up reactions on ${ }^{7} \mathrm{Li}$. 
Table B.2: Relative S-factors for proton pickup reactions on ${ }^{12} \mathrm{C}$

\begin{tabular}{c|c|c|c}
\hline \hline Reaction & $J^{\pi}$ & Ex $[\mathrm{MeV}]$ & S-factor \\
\hline${ }^{12} \mathrm{C}(\mathrm{p}, 2 \mathrm{p})^{11} \mathrm{~B}$ & $3 / 2^{-}$ & 0.00 & 2.00 \\
$E_{p}=98.7[\mathrm{MeV}]$ & $1 / 2^{-}$ & 2.12 & 0.37 \\
{$[110]$} & $5 / 2^{-}$ & 4.44 & 0.15 \\
& $3 / 2^{-}$ & 5.02 & 1.08 \\
& $1 / 2^{+}$ & 6.79 & 0.25 \\
\hline${ }^{12} \mathrm{C}\left(\mathrm{d},{ }^{3} \mathrm{He}\right)^{11} \mathrm{~B}$ & $3 / 2^{-}$ & 0.00 & 2.98 \\
$E_{d}=52[\mathrm{MeV}]$ & $1 / 2^{-}$ & 2.12 & 0.69 \\
{$[111]$} & $3 / 2^{-}$ & 5.02 & 0.31 \\
\hline${ }^{12} \mathrm{C}\left(\mathrm{e}, \mathrm{e}^{\mathrm{p}}\right)^{11} \mathrm{~B}$ & $3 / 2^{-}$ & 0.00 & 1.72 \\
$300<E_{e}<500$ & $1 / 2^{-}$ & 2.12 & 0.26 \\
{$[\mathrm{MeV}][112]$} & $3 / 2^{-}$ & 5.02 & 0.20 \\
\hline
\end{tabular}

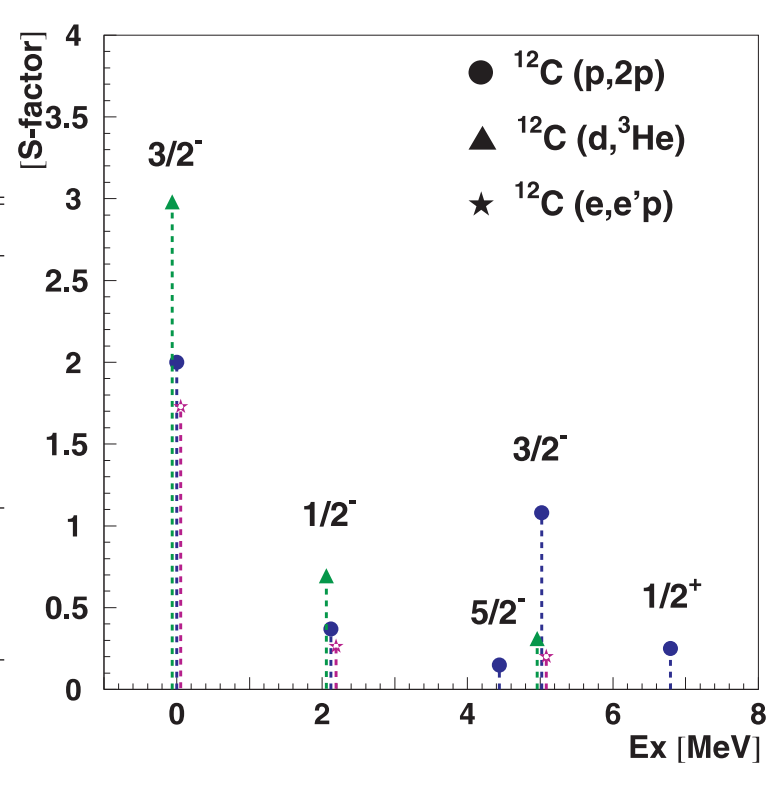

Figure B.2: Relative spectroscopic factors for proton pick-up reactions on ${ }^{12} \mathrm{C}$. 


\section{Appendix C}

\section{Additional information of the water Čerenkov counter}

\section{C.1 Aging test for number of photoelectron}

A life time chemical stability has been tested for the wave length shifter, 30 and $50 \mathrm{mg} / l$ Amino G-salt with cosmic rays. Fig. C.1 shows a schematic of the bench test. Two different density $(30,50 \mathrm{mg} / l)$ of water Čerenkov counters were placed between two scintillation counters vertically separated by $60 \mathrm{~cm}$. The number of photoelectrons, calculated by sum of each two phototube, was recorded periodically for 73 days. Fig. C.2 shows a time decay of number of photoelectrons for 30 (rectangle plot) and $50 \mathrm{mg} / l$ (circle plot) Amino G-salt water Cerenkov counters. The solid (dashed) line was derived by $\chi^{2}$ fitting for number of photoelectrons of $50(30) \mathrm{mg} / \mathrm{l}$ counter and decay rate of number of photoelectrons were estimated as 0.0550 (0.1154) \%/day respectively.

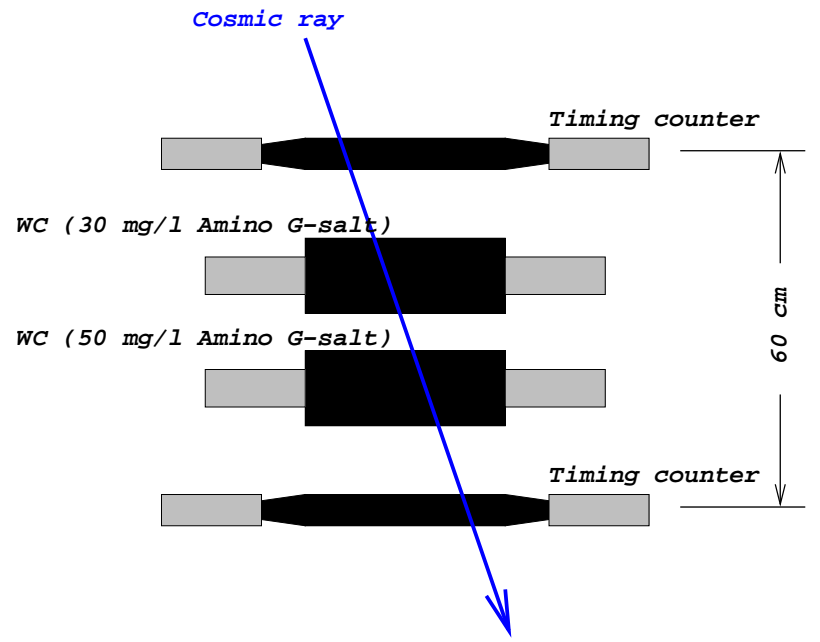

Figure C.1: Schematic of cosmic ray bench test for chemical stability test. 


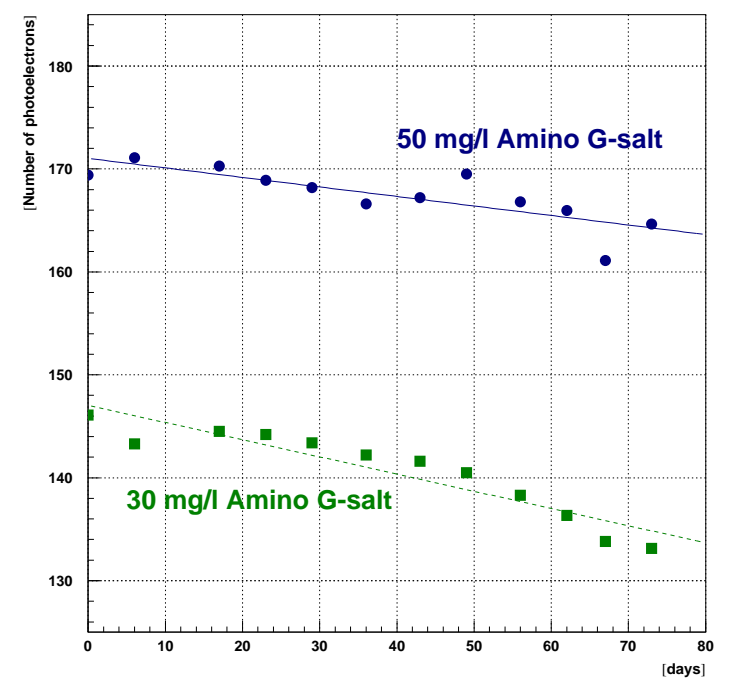

Figure C.2: Life time of the number of photoelectrons for 30 (rectangle) and 50 (circle) $\mathrm{mg} / \mathrm{l}$ Amino G-salt water Cerenkov counter. $\chi^{2}$ fitting has been done for $30(50) \mathrm{mg} / l$ density as dashed (solid) line. 


\section{Appendix D}

\section{Systematic errors of the ${ }_{\Lambda}^{12} \mathbf{B}$ cross section}

The cross section of the ${ }_{\Lambda}^{12} \mathrm{~B}$ ground state doublet is measured to be $54 \pm 10 \pm{ }_{22}^{25} \mathrm{nb} / \mathrm{sr}$ by fitting with a single Gaussian for a prominent peak marked as \#1 in Fig. D.1 (a).

The systematic errors are evaluated to be $54 \times 0.22 \cong 12 \mathrm{nb} / \mathrm{sr}$ from the factors listed in Table 4.10.

Here, we assumed that the accidental background can be exactly subtracted as described in Chapter 5, although there is some excess of events at $-11<-B_{\Lambda}<-3 \mathrm{MeV}$ between the prominent peaks. This background subtraction is justified because there should be some intensity resulting from the core excited states existence between $s$ and $p$-shell states, though none of clear peak structures can be recognized there.

However, the following two possibilities cannot be excluded, which causes larger systematic errors of the cross section.

(a) The existence of a continuous background between $s$ and $p$-shell states, resulting from some sort of unknown reasons, cannot be completely excluded. In order to estimate a lower limit of the cross section, the minimum cross section of the ground state doublet is evaluated by fitting after subtracting the continuous background as shown in Fig. D.1 (b). The background is fitted with the 1st order of linear function.

The systematic error of the cross section is determined to be $-22 \mathrm{nb} / \mathrm{sr}$.

(b) A tail can be recognized beside the ground state doublet peak around $-13.5<-B_{\Lambda}<$ $-12 \mathrm{MeV}$ as shown in Fig. D.1 (c).

There is a possibility that the events in the tail belongs to the ground state doublet. Therefore, the tail is fitted with a linear function and the area in the tail is evaluated and added to the systematic error of the cross section for the ground state doublet.

The systematic error is found to be $+25 \mathrm{nb} / \mathrm{sr}$. 


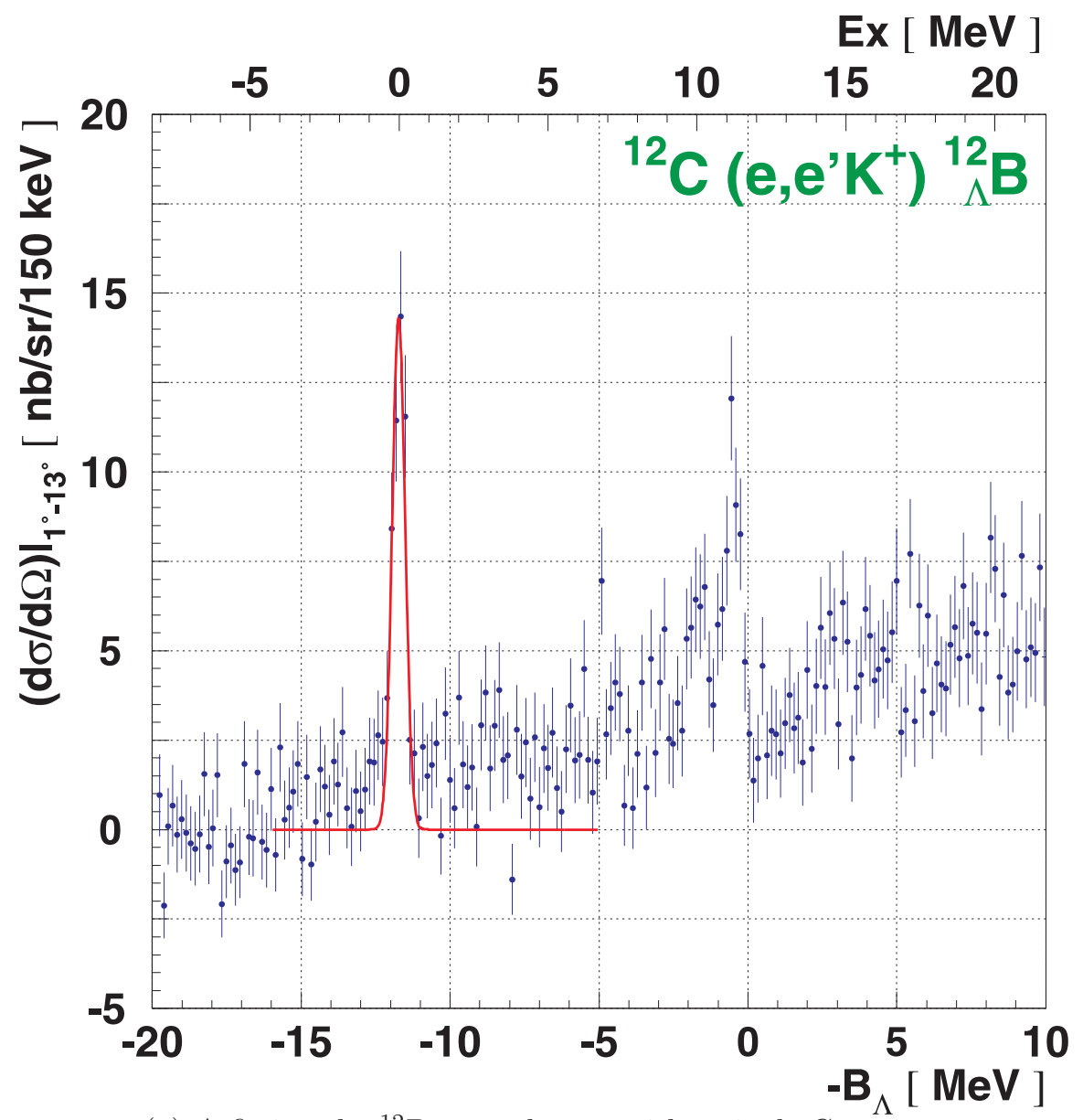

(a) A fitting the ${ }_{\Lambda}^{12} \mathrm{~B}$ ground state with a single Gaussian.

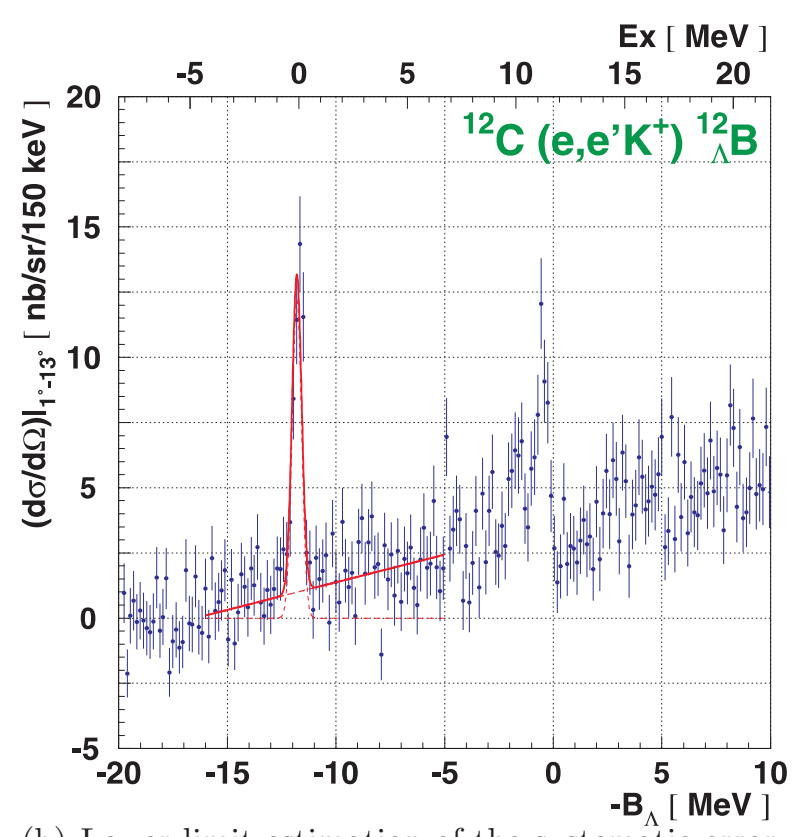

(b) Lower limit estimation of the systematic error.

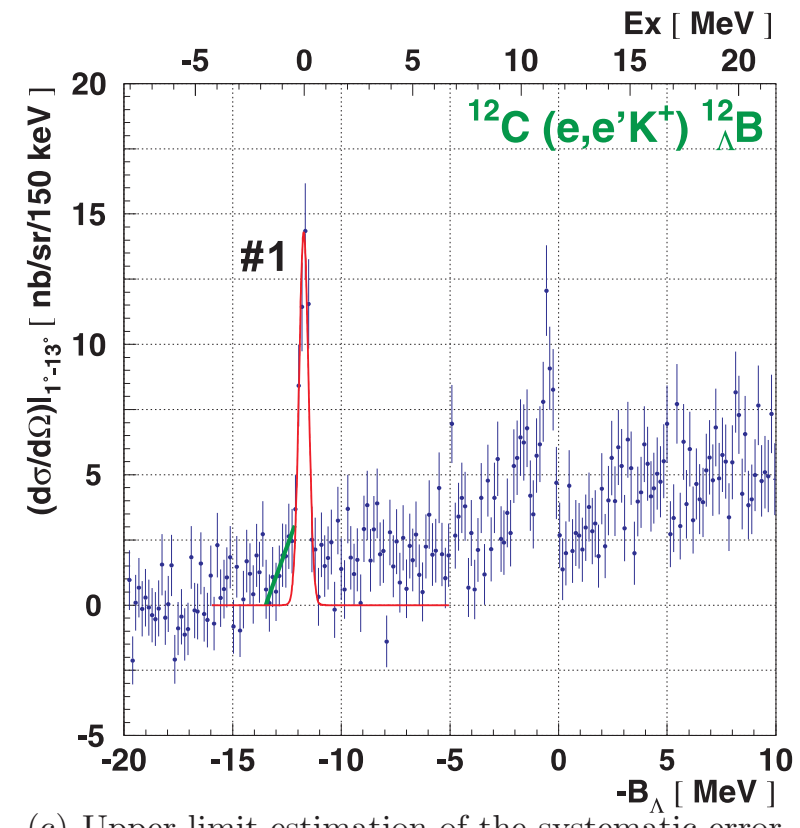

(c) Upper limit estimation of the systematic error.

Figure D.1: The systematic errors estimation of the cross section for the ${ }_{\Lambda}^{12} \mathrm{~B}$ ground state doublet. 


\section{Bibliography}

[1] H. Bandō et al. Phys. Rev. C 31(1985) 265.

[2] D.J. Millner et al. Phys. Rev. C 38(1988) 2700.

[3] M. Rayet. Nucl. Phys. A367(1981) 381.

[4] C. Bennhold et al. in: L. Tang, O. Hashimoto (Eds), Proc. JLab Workshop on Hypernuclear Physics with Electromagnetic Probes, HYPJLAB99, 2-4 December, Hampton, USA, 1999, p1.

[5] E.H. Auerbach et al. Ann. Phys. 148(1983) 381.

[6] S. Cohen and D Kurath. Nucl. Phys. 73(1965) 1; Nucl. Phys. A101(1967)1.

[7] Th.A. Rijken et al. Phys. Rev. C 59(1999) 21.

[8] R.H. Dalitz and A. Gal. Ann. Phys. 116(1978) 167.

[9] D.J. Millner et al. Phys. Rev. C 31(1985) 499.

[10] C.B. Dover. LAMPF Workshop on $(\pi, \mathrm{K})$ Physics: Los Alamos, NM 1990, API Conf.Proc.224.

[11] Y. Yamamoto and H. Bandō. Prog. Theor. Phys. 73(1985) 905.

[12] Y. Yamamoto et al. Progr. Theoret. Phys. Suppl. 117(1994) 361.

[13] M. Danysz et al. Phli. Mag. 44(1953) 348.

[14] D.H. Davis et al. Nucl. Phys. A547(1992) 269c.

[15] A. Bamberger et al. Phys. Lett. B36 (1971) 412.

[16] M.A. Faessler et al. Phys. Lett. B46 (1973) 468.

[17] W. Brückner et al. Phys. Lett. B55 (1975) 107.

[18] W. Brückner et al. Phys. Lett. B62 (1976) 481.

[19] W. Brückner et al. Phys. Lett. B79 (1978) 157.

[20] R. Bertini et al. Phys. Lett. B83 (1979) 306.

[21] R. Bertini et al. Nucl. Phys. A360 (1981) 315.

[22] R. Bertini et al. Nucl. Phys. A368 (1981) 365. 
[23] R.E. Chrien et al. Phys. Lett. B89 (1979) 31.

[24] M. May et al. Phys. Rev. Lett. 47 (1981) 1106.

[25] C. Milner et al. Phys. Rev. Lett. 54 (1985) 1237.

[26] P.H. Pile et al. Phys. Rev. Lett. 66 (1991) 2585.

[27] M. Akei et al. Nucl. Phys. A534 (1991) 478.

[28] T. Hasegawa et al. Phys. Rev. Lett. 74 (1995) 224.

[29] T. Hasegawa et al. Phys. Rev. C 53(1996) 1210.

[30] O. Hashimoto et al. Nucl. Phys. A 639(1998) 93c.

[31] T. Hotchi et al. Phys. Rev. C 64(2001) 044302.

[32] K. Itonaga, T. Motoba, and H. Bandō. Progr. Theoret. Phys. Suppl. 84(1990) 291.

[33] K. Itonaga, T. Motoba, O. Richter, and M. Sotona. Phys. Rev. C 49(1994) 1045.

[34] M. May et al. Phys. Rev. Lett. 51(1983) 2085.

[35] M. May et al. Phys. Rev. Lett. 78(1997) 4343.

[36] H. Tamura et al. Phys. Rev. Lett. 84(2000) 5963.

[37] K. Tanida et al. Phys. Rev. Lett. 86(2001) 1982.

[38] K. Tanida et al. Nucl. Phys. A 721(2003) 999c.

[39] K. Miwa et al. Nucl. Phys. A 754(2005) 80c.

[40] Y. Miura et al. Nucl. Phys. A 754(2005) 75c.

[41] Y. Miura et al. Ph.D Thesis.

[42] H. Akikawa et al. Phys. Rev. Lett. 88(2002) 082501.

[43] M. Ukai et al. Phys. Rev. Lett. 93(2004) 232501.

[44] H. Tamura et al. Nucl. Phys. A 754(2005) 58c.

[45] T. Miyoshi et al. Phys. Rev. Lett. 90(2003) 232502.

[46] L. Yuan et al. Phys. Rev. C 73(2006) 044607.

[47] H. Tamura O. Hashimoto. Prog. Part. Nucl. Phys. 57(2006) 564.

[48] C.B. Dover et al. Phys. Rev. C 22(1980) 2073.

[49] T. Motoba et al. Progr. Theoret. Phys. Suppl. 117(1994) 123.

[50] H. Bandō et al. Int. Journal of Modern Phys. A5(1990) 4021.

[51] J. Adam et al. Czech. J. Phys. 42(1992) 1167. 
[52] S. Nozawa et al. Nucl. Phys. A513(1990) 511.

[53] B. R. Martin et al. Nucl. Phys. B94(1975) 413.

[54] C. Yan et al. Nucl. Instr. and Meth. A365(1995) 261.

[55] SAPHIR Collaboration. Phys. Lett. B455(1998) 20.

[56] C.E. Hyde-Wight et al. Electron scattering at $0^{\circ}:$ A photon tagging technique.

[57] M. Jurič et al. Nucl. Phys. B52(1973) 1.

[58] T. Motoba et al. in: K. Maeda, H. Tamura, S. N. Nakaura, and O. Hashimoto (Eds), Electroproduction of strangeness on Nucleons and Nuclei, SENDAI03, (2004)221, World Scientific.

[59] Group3. DTM-151 DIGITAL TESLAMETER with serial communications USER'S MANUAL. Group3 Technology Ltd., 2 Charann Place, Avondale, Auckland 1007, New Zealand.

[60] J.E. Spencer et al. Nucl. Instr. and Meth. 49(1967) 181.

[61] S. Kowalski et al. RAYTRACE. Laboratry for Nuclear Science and Department of Physics, Massachusetts Institute Of Technology, Cambridge, Massachusetts 02139 U.S.A, May 161986.

[62] L. Yuan. High precision hypernuclear spectroscopy study by the (e, e'K $\left.{ }^{+}\right)$reaction. Ph.D. dissertation, Graduate College of Hampton University, 2002.

[63] M. Carl et al. Nucl. Instr. and Meth. A527(2004) 301.

[64] Jr.J.W. Lightbody and J.S. O'Connell. Computers in Physics 57 May/June 1988.

[65] H. Yamazaki et al. Phys. Rev. C 52(1995) 1157.

[66] D. Dutta. Radiative corrections - the simc way. Internal Hall C note.

[67] E. Miyata et al. Nucl. Instr. Meth. A459 (2001) 157.

[68] M. Nomachi et al. Serial data link on advanced tca back plane. in: Proc. Real time Conference 2005 14th IEEE-NPSS, 4-10 June 2005.

[69] H. Nomura. Master's thesis (in Japanese), Dept. of Phys. Tohoku Univ., 2003.

[70] V. Gyurjyan et al. Jefferson lab data acquisition run control system. in: Proc. CHEP04, 29 September 2004, Interlaken, Switzerland.

[71] S.N. Nakamura et al. Nucl. Phys. A 754(2005) 421c.

[72] SHIMADZU. http://www. shimadzu.com/.

[73] N.A. Porter. Il Nuovo Cim. 5 (1957) 526.

[74] P. Némethy et al. Nucl. Instr. Meth. 173 (1980) 251.

[75] A. Bezague et al. Nucl. Instr. Meth. 158 (1979) 303. 
[76] D.F. Geesaman et al. Hall C Software Vade Mecum v1.3(unpublished)1994.

[77] T. Bowen et al. Phys. Rev. D 7(1973) 22.

[78] R.W. Bland et al. Nucl. Phys. B 13(1969) 595.

[79] D.V. Bugg et al. Phys. Rev. 168(1968) 1466.

[80] G. Giacomelli et al. Nucl. Phys. B 20(1970) 301.

[81] K. Abe et al. Phys. Rev. D 11(1975) 1719.

[82] J. Phys. G : Nucl. Part. Phys. 33(2006) 43.

[83] T. Miyoshi et al. Ph.D Thesis.

[84] M. Iodice et al. Phys. Rev. Lett. 99(2007) 052501.

[85] M. Sotona. Private communication.

[86] S. Cohen and D. Kurath. Nucl. Phys. A101(1967) 1.

[87] P. G. Roos et al. Nucl. Phys. A255(1975) 187.

[88] Th. A. Rijken et al. Nucl. Phys. A547(1992) 245c.

[89] T. Motoba. Mesons and Light Nuclei: 8th conference, edited by J. Adam et al.,(2001)125.

[90] T. Mizutani et al. Phys. Rev. C58(1998) 75.

[91] W.A. Williams et al. Phys. Rev. C46(1992) 1617.

[92] T. Mart et al. Phys. Rev. C61(1999) 012201.

[93] K.S. Myint and Y. Akaishi. Progr. Theoret. Phys. Suppl. 146(2002) 599.

[94] R. H. Dalitz et al. Nucl. Phys. B47(1972) 109.

[95] Y. Akaishi et al. Phys. Rev. Lett. 84(2000) 3539.

[96] B.F. Gibson et al. Phys. Rev. C37(1988) 679.

[97] E. Hiyama et al. Phys. Rev. C65(2001) 011301.

[98] A. Nogga. Nucl. Phys. A754(2005) 36c.

[99] E. Hiyama et al. Private communication, to be published.

[100] H. Tamura et al. Phys. Lett. B84(2000) 5963.

[101] E. Hiyama et al. Phys. Rev. C59(1999) 2351.

[102] O. Richter et al. Phys. Rev. C 43(1991) 2753.

[103] L. Majling et al. Phys. Lett. B92(1980) 256.

[104] O. Richter et al. Phys. Rev. C43(1991) 2753. 
[105] E. Hiyama et al. Phys. Rev. C 53(1996) 2075.

[106] E. Hiyama et al. Nucl. Phys. A639(1998) 173c.

[107] G. Audi et al. Nucl. Phys. A 729(2003) 337.

[108] N.M. Clarke. J. Phys. G: Nucl. Part. Phys. 18(1992) 917.

[109] L. Lapikás et al. Phys. Rev. Lett. 82(1999) 4404.

[110] F. Ajzenberg-selove. Nucl. Phys. A433(1985) 1.

[111] G. Mairle et al. Nucl. Phys. A253(1985) 253.

[112] A.E.L. Dieperink et al. Annu. Rev. Nucl. Part. Sci. 40(1990) 239. 